\title{
Hereditary Hearing Impairment
}

\section{Clinical and Genetic Aspects in DFNA5, DFNA9 and DFNA11}

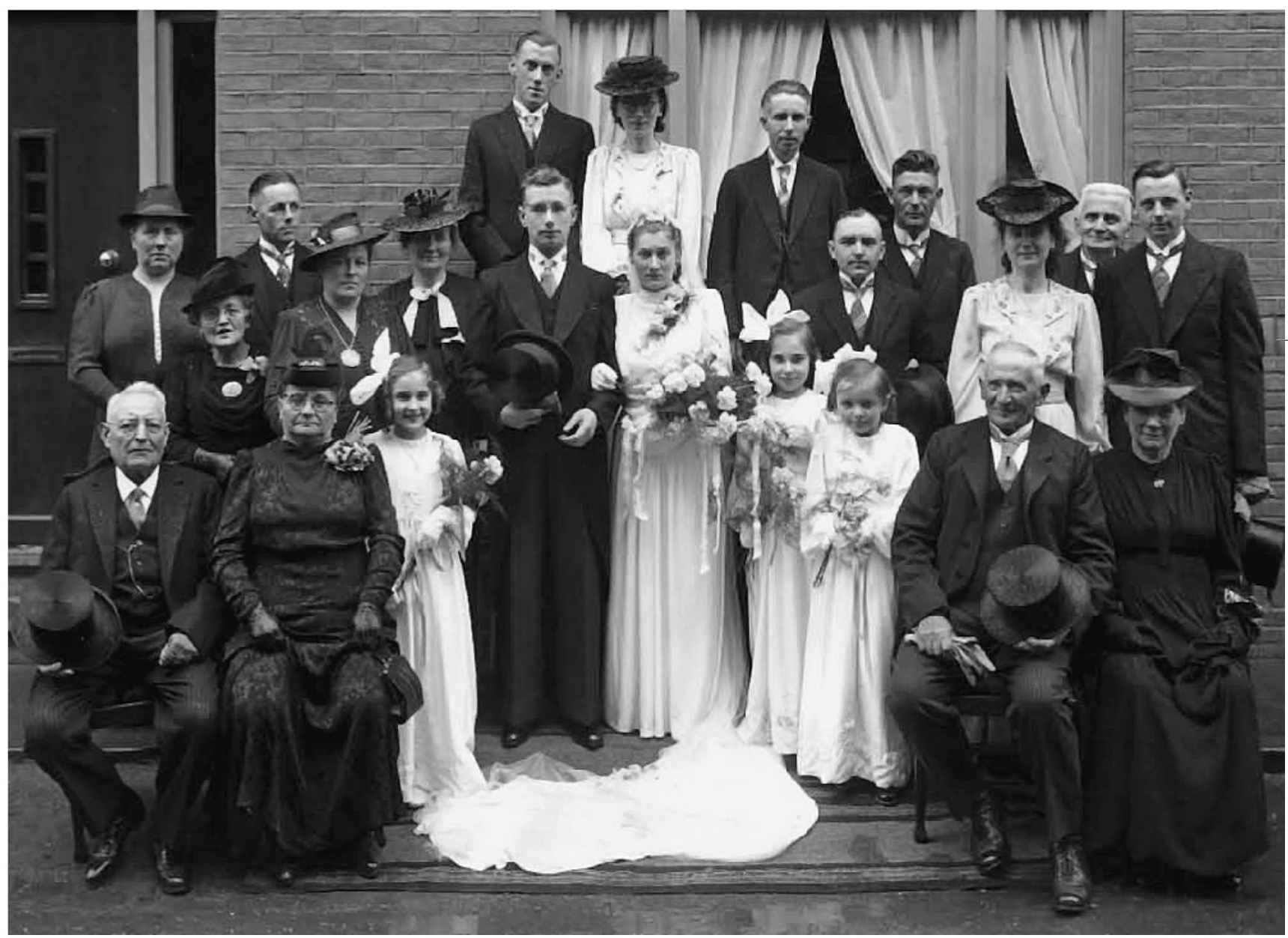




\title{
Hereditary Hearing Impairment
}

\author{
Clinical and Genetic Aspects in \\ DFNA5, DFNA9 and DFNA11
}

Anne M.L.C. Bischoff 
Print: $\quad$ Drukkerij Graficolor Nijmegen

Lay-out: Diny Helsper

Cover: $\quad$ Foto uit familie-archief

ISBN: 978-90-9021807-6

(C) A.M.L.C. Bischoff

Hereditary Hearing Impairment. Clinical and Genetic Aspects in DFNA5, DFNA9 and DFNA11

Thesis Radboud University Nijmegen Medical Centre, Nijmegen.

All rights reserved. No part of this publication may be reproduced in any form or by any means, electronically, mechanically, by print or otherwise without written permission of the copyright owner. 


\section{Hereditary Hearing Impairment}

\section{Clinical and Genetic Aspects in DFNA5, DFNA9 and DFNA11}

Een wetenschappelijke proeve op het gebied van de

Medische Wetenschappen

Proefschrift

ter verkrijging van de graad van doctor

aan de Radboud Universiteit Nijmegen

op gezag van de rector magnificus prof. mr. S.C.J.J. Kortmann, volgens besluit van het College van Decanen

in het openbaar te verdedigen op

vrijdag 15 juni 2007

om 10.30 uur precies

door

Anne Maria Louisa Carolina Bischoff

geboren op 21 januari 1977

te Rotterdam 
Promotores:

prof. dr. C.W.R.J. Cremers

prof. dr. J.R.M. Cruysberg

Copromotores:

\author{
dr. H. Kremer \\ dr. P.L.M. Huygen
}

Manuscriptcommissie:

Prof. dr. V.V.A.M. van Slobbe-Knoers

Prof. dr. P.H. Van de Heyning

Prof. dr. J.J.E. Keunen

Publication of this thesis was financially supported by:

Stichting ATZE Spoor Fonds, Researchfonds Oogheelkunde, Altana Pharma B.V., Artu Biologicals, Atos Medical, Bayer, Beter Horen, Electro Medical Instruments, GlaxoSmithKline, Schering-Plough, Schoonenberg Hoorcomfort, UCB Pharma. 


\section{Table of Contents}

Chapter 1 Introduction 9

$\begin{array}{lll}\text { Chapter } 2 \text { DFNA5 } & 47\end{array}$

2.1 A novel mutation identified in the DFNA5 gene in a 49

Dutch family: A clinical and genetic evaluation.

AMLC Bischoff, MWJ Luijendijk, PLM Huygen, G van Duijnhoven, EMR De

Leenheer, GG Oudesluijs, L Van Laer, FPM Cremers, CWRJ Cremers, H Kremer

Audiology \& Neuro-Otology 2004;9:34-46.

Chapter 3 DFNA9

3.1 Vestibular deterioration precedes hearing deterioration in the 73 P51S COCH mutation (DFNA9): An analysis in 74 mutation carriers. AMLC Bischoff, PLM Huygen, MH Kemperman, RJE Pennings, SJH Bom, WIM Verhagen, RJC Admiraal, H Kremer, CWRJ Cremers

Otology \& Neurotology 2005;26:918-925.

3.2 Vertical Corneal Striae in Families with Autosomal Dominant Hearing Loss: DFNA9/COCH.

AMLC Bischoff, RJ Pauw, PLM Huygen, AL Aandekerk, H Kremer, CWRJ

Cremers, JRM Cruysberg

American Journal Ophthalmology 2007, in press.

Chapter 4 DFNA11

4.1 Cochleovestibular and ocular features in a

Dutch DFNA11 family.

AMLC Bischoff, RJE Pennings, PLM Huygen, Luijendijk MWJ,

E van Wijk, JRM Cruysberg, H Kremer, CWRJ Cremers

Otology \& Neurotology 2006;27:323-331. 
Chapter 5 Discussion and Summary

Chapter 6 Samenvatting

Dankwoord

Curriculum Vitae

List of abbreviations 
Take the first step in faith.

You don't have to see the whole staircase.

Just take the first step.

Martin Luther King 



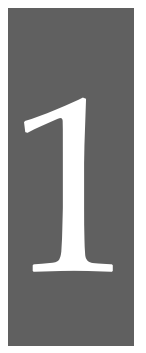

\section{Introduction}





\section{Introduction}

At present, hearing impairment is the most common perception disorder. ${ }^{1,2}$ Causes of hearing impairment are wide-ranging and include genetic aberrations, perinatal infection, noise, ototoxic drugs and cochlear trauma. Hearing loss can arise before speech development (prelingual) or afterwards (postlingual). The frequency of early childhood sensorineural hearing impairment is estimated to be 1 in 650 newborns. $^{3-5}$ More than half of the prelingual cases are genetically determined and about $75 \%$ of those are accounted for by non-syndromic forms ${ }^{3,4,6}$. Far more people are affected by postlingual hearing impairment than by prelingual hearing impairment. Environmental factors and aging play important roles. Several monogenic forms also exist and they mostly follow an autosomal dominant pattern of inheritance. Non-syndromic hearing impairment can be further categorized into types of inheritance: approximately $77 \%$ of the cases are autosomal recessive, $22 \%$ are autosomal dominant, $1 \%$ are $X$-linked and $<1 \%$ are mitochondrial. ${ }^{4}$ Autosomal dominant loci have been designated with the prefix "DFNA", recessive loci with "DFNB", X-linked loci with "DFN" and modifying loci with "DFNM". Y-linked inheritance has been proposed in one Chinese family, which has been designated DFNY1. ${ }^{7}$

Since the first successful attempts to detect hearing impairment loci and genes in 1992, rapid progress has been made. By now, 54 loci and 21 genes have been identified in non-syndromic autosomal dominant types of hearing impairment. An overview of the human hearing impairment loci and genes can be found on the Hereditary Hearing Loss Homepage (Van Camp G, Smith RJH. Hereditary Hearing Loss Homepage. URL: http:// webhost.ua.ac.be/hhh/).

The complexity of hereditary hearing impairment is demonstrated by the involvement of various genes in the dominant and recessive non-syndromic forms and also in the non-syndromic and syndromic forms. For example, mutations in the myosin VIIa gene $(M Y O 7 A)$ can cause DFNA11, ${ }^{8,9}$ DFNB2, ${ }^{10,11}$ Usher Type $1 \mathrm{~B}^{12}$ and atypical Usher syndrome. . $^{13,14}$

\section{Historical overview}

Systematic clinical research into hereditary hearing impairment started in the mid $19^{\text {th }}$ century ${ }^{15,16}$. Two patterns of inheritance were identified: direct (later called autosomal dominant) and indirect (or autosomal recessive). ${ }^{15,17,18}$ Mendel's laws $^{19}$ were rediscovered early in the $20^{\text {th }}$ century, but for many years it had been understood that there was a relationship between autosomal recessive inherited diseases and the degree of consanguinity. As the latter was fairly high in the $19^{\text {th }}$ century (about 30\% in some areas), the genetic disadvantages of consanguineous 
marriages became well documented. ${ }^{16,17}$ The earliest reports on the most frequently occurring hereditary hearing impairment syndromes appeared at the turn of the century. In 1922, Albrecht was the first to write about autosomal dominant hearing impairment without any associated abnormalities. ${ }^{20}$

In the nineteen sixties and the seventies, encyclopaedic books were launched on hereditary hearing impairment syndromes. ${ }^{21-26}$ About ten years later, gene linkage studies were initiated to trace the loci for the genes involved in syndromic hearing impairment. ${ }^{27}$ Shortly afterwards, the non-syndromic forms were addressed and the first successful linkage was accomplished in Nijmegen for the X-linked progressive mixed deafness syndrome with perilymphatic gusher during stapes surgery. ${ }^{28,29}$ The associated features in this disease were mostly subclinical, which led to it being categorized as non-syndromic DFN3. Clearly, the X-linked pattern of inheritance facilitated this locus identification. ${ }^{28}$

In the past ten years, there has been an enormous increase in the identification of loci and genes owing to progress in the Human Genome Project and the availability of cochlear-specific cDNA libraries and mouse models for hearing impairment. These developments in human genetics encouraged the Nijmegen ORL department to extend its genetic research towards non-syndromic autosomal dominant hearing impairment, initially in collaboration with the Department of Medical Genetics of the University of Antwerp and, since 2000, principally with the Otogenetic Department of the Radboud University Nijmegen Medical Centre. Many contributions have been made to describe the phenotypes and genotypes of a series of autosomal dominant forms of hearing impairment, especially DFNA2, DFNA4, DFNA5, DFNA6/14, DFNA8/12, DFNA9, DFNA10, DFNA11, DFNA13, DFNA20/26, DFNA21 and DFNA31. This thesis was part of the ongoing Nijmegen otogenetic research projects and focused on the clinical and genetic headway regarding DFNA5, DFNA9 and DFNA11.

\section{Clinical Classification}

Only a few clinical criteria were available to differentiate between types of hereditary hearing impairment until the relevant loci and genes were identified. Hearing impairment was classified not only on the basis of features of conductive or mixed hearing loss, but also on age of onset, severity and progression of hearing impairment, unilaterality or bilaterality of hearing impairment, configuration of the audiograms and the presence or absence of vertigo. The shape of the audiogram was a very important characteristic, because it could distinguish hearing impairment into low, mid, high and all frequency deficits. Consequently, 16 types have been described in "Genetic and Metabolic Deafness" 
by Konigsmark and Gorlin ${ }^{24}$. In the years that followed, so many new types of hereditary hearing impairment were identified that the existing nomenclature had to be further categorized into DFNA, DFNB, DFN and DFNM, as mentioned above.

Table 1 presents the genotype of the different DFNA types. As so many of the numerous autosomal dominant types are fairly rare, it is of value to use the clinical criteria to predict which type might be involved in a given trait, in order to perform linkage to a specific locus or mutation analysis on a specific gene. To facilitate this, the method of Age Related Typical Audiograms (ARTA) was introduced. ${ }^{30}$ This tool can be used to make phenotype "fingerprints", so that it becomes possible to test whether the phenotype of a new family is significantly different from a known DFNA type. ${ }^{30}$ Huygen et al. ${ }^{31}$ used a phenotypic classification with five categories, similar to the one described by Mazzoli et al.: ${ }^{32}$ high-frequency, mid-frequency, and low-frequency hearing impairment, hearing impairment at all frequencies and residual hearing. In non-syndromic autosomal dominant types of hearing impairment, high-frequency hearing impairment (downsloping audiogram) is present in DFNA2, DFNA3, DFNA5, DFNA7, DFNA8/12, DFNA9, DFNA15, DFNA16, DFNA17, DFNA20/26, DFNA23, DFNA24, DFNA30, DFNA36, DFNA42, DFNA47 and DFNA48. Mid-frequency hearing impairment phenotypes (U-shaped audiogram) are present in DFNA8/12, DFNA13, DFNA44 and DFNA49. Low-frequency hearing impairment is present in DFNA1, DFNA6/14/38 and DFNA54, while hearing impairment at all frequencies (flat audiogram) is present in DFNA4, (DFNA8/12), DFNA10, DFNA11, DFNA18, DFNA21, DFNA25, DFNA28, DFNA31, DFNA41, DFNA43 and DFNA50. Residual hearing is only seen in DFNA22. ${ }^{31}$

Other clinical characteristics are also useful, such as age of onset, progression and severity of the hearing loss, intra-familial variability, speech recognition scores, presence or absence of vestibular dysfunction and tinnitus and radiographically detectable cochleovestibular anomalies. Therefore, the search for the locus can be guided by these clinical data and can be limited to some well-known DFNA types before employing the more costly approach of a total genome scan to identify the genetic defect.

An overview is given of DFNA5, DFNA9 and DFNA11 in the following part of this introduction, because these three DFNA types formed the focus of this thesis. 
Table 1. Description of phenotype, gene linkage and gene identification

\begin{tabular}{|c|c|c|c|c|c|}
\hline Locus & $\begin{array}{l}\text { Relevant Clinical } \\
\text { Description }\end{array}$ & $\begin{array}{l}\text { Number of } \\
\text { Families* }\end{array}$ & $\begin{array}{l}\text { Site of Mutation or } \\
\text { Amino Acid Change }\end{array}$ & Gene & Localization \\
\hline DFNA1 & $\begin{array}{l}\text { León } 1981, \mathrm{CR}^{33^{\star}} \\
\text { Lalwani } 1998^{34}\end{array}$ & 1 Costa Rican 35 & c.IVS17+1 G>T35 & DIAPH1 ${ }^{35}$ & $5 q 31^{36}$ \\
\hline \multirow[t]{2}{*}{ DFNA2 } & $\begin{array}{l}\text { Marres 1997, NL } \mathrm{NL}^{3{ }^{\star}} \\
\text { Kunst } 1998^{38} \\
\text { Ensink 200039 } \\
\text { Akita 200140 } \\
\text { De Leenheer } 2002^{41,42} \\
\text { Stern 200343 } \\
\text { Topsakal 2005 }\end{array}$ & $\begin{array}{l}15 \\
1 \text { Belgian }^{45} \\
1 \text { Japanese } \\
1 \text { Dutch } \\
47 \\
5 \text { Dutch, }^{46} \\
\text { Japanese }^{40,44,45,48} \\
1 \text { USA }^{49} \\
1 \text { French }^{50} \\
2 \text { USA, French }^{45} \\
1 \text { Dutch }^{45}\end{array}$ & $\begin{array}{l}\text { c.211_223del }{ }^{45} \\
\text { c.211delC } \\
\text { p.Leu274His } \\
\text { p.Trp276Ser } \\
\\
\text { p.Leu28144,45er }{ }^{49} \\
\text { p.Gly285Ser } \\
\text { p.Gly285Cys } \\
\text { p.Gly321Ser }\end{array}$ & $\mathrm{KCNQ} 4^{50}$ & $1 \mathrm{p} 34^{48,51}$ \\
\hline & Lacking clinical data & $\begin{array}{l}1 \text { Chinese }^{52} \\
1 \text { Chinese }^{52}\end{array}$ & $\begin{array}{l}\text { p.Glu183Lys }{ }^{52} \\
\text { p.Arg180TER }\end{array}$ & GJB352 & $1 \mathrm{p} 35.1^{51,52}$ \\
\hline \multirow[t]{2}{*}{ DFNA3 } & $\begin{array}{l}\text { Tekin 2001, USA } \\
\text { Denoyelle } 2002^{54} \\
\text { Primignani } 2003^{55}\end{array}$ & $\begin{array}{l}14 \\
1 \text { UK }{ }^{56} \\
3 \text { French/USA } \\
13,57 \\
1 \text { Italian }^{58} \\
1 \text { Egyptian } \\
2 \text { French/Italian }^{60,61} \\
1 \text { Austria/Czech }^{62} \\
1 \text { Chinese }^{63} \\
1 \text { Italian } \\
1 \text { French }^{64} \\
1 \text { Chinese }^{63}\end{array}$ & $\begin{array}{l}\text { p.Met34Thr } 56 \wedge \\
\text { p.Trp44Cys }{ }^{53,57} \\
\text { p.Thr55Asn }{ }^{58} \\
\text { p.Arg75Trp } 59 \\
\text { p.Arg75Gln } \\
\text { p.Arg14361 } \\
\text { p.Ala171Thr }{ }^{62} \\
\text { p.Asp179Asn } \\
\text { p.Cys202Phe } \\
\text { c.299_300delAT }\end{array}$ & GJB256 & $13 q 11-12^{65}$ \\
\hline & Lacking clinical data & 1 Italian $^{66}$ & p.Thr5Met ${ }^{66}$ & GJB666 & $13 q 12^{67}$ \\
\hline DFNA4 & $\begin{array}{l}\text { Pusch 2004, DE } \\
\text { Yang 2005* }\end{array}$ & $\begin{array}{l}9 \\
1 \text { German }^{70} \\
1 \text { German }^{69} \\
\text { sporadic case }^{70} \\
1 \text { Belgian }^{70} \\
1 \text { Italian }^{70}\end{array}$ & $\begin{array}{l}\text { p.Ser7X }{ }^{70} \\
\text { p.Ser120Leu } \\
\text { p.Gly376Cys } \\
\text { p.Arg726Ser } \\
\text { p.Leu976Phe } \\
\text { p. }\end{array}$ & MYH1470 & $19 q 13.33^{68,71}$ \\
\hline DFNA5 & $\begin{array}{l}\text { Huizing } 1966, \mathrm{NL}^{72^{\star}} \\
\text { Huizing } 1983^{73} \\
\text { De Leenheer } 2002^{74} \\
\text { Bischoff } 2005^{75}\end{array}$ & $\begin{array}{l}3 \\
1 \text { Dutch }^{76} \\
1 \text { Dutch }^{75} \\
1 \text { Chinese }^{77}\end{array}$ & $\begin{array}{l}\text { c.IVS7 } 1189 \mathrm{del} / 127 \text { ins }^{76} \\
\text { c.IVS7-6C>G }>\mathrm{G}^{65} \\
\text { c.IVS7-22_-20delCTT }{ }^{77}\end{array}$ & DFNA5 ${ }^{76}$ & $7 \mathrm{p} 15^{78}$ \\
\hline $\begin{array}{l}\text { DFNA6/14/ } \\
\text { DFNA38 }\end{array}$ & $\begin{array}{l}\text { Vanderbilt gr. ' } 68, \text { USA }^{79 \star} \\
\text { Kunst } 19999^{80} \\
\text { Bom } 2002^{81} \\
\text { Lesperance } 2003^{82} \\
\text { Pennings } 2003^{83}\end{array}$ & $\begin{array}{l}23-26 \\
1 \text { isolated person } \\
1 \text { Japanese } \\
1 \text { Dutch }^{85} \\
1 \text { Dutch }^{84} \\
2 \text { German/Dutch }^{84,86} \\
1 \text { German } \\
3 \text { Canadian/ } \\
\text { Dutch/USA } \\
1 \text { Dutch }^{83} \\
1 \text { Swiss }^{89} \\
1 \text { USA }^{86} \\
1 \text { UK }^{84} \\
1 \text { USA }^{86} \\
2 \text { USA }^{84,86}\end{array}$ & $\begin{array}{l}\text { p.Lys193Gln } \\
\text { p.Lys634Thr } 85 \\
\text { p.Gly674Glu } \\
\text { p.Gly674Val } \\
\text { p.Thr699Met } \\
\text { p. } \\
\text { p.Lys705Asn } \\
\text { p.Ala716Thr } \\
\\
\text { p.Ile767del } \\
\text { p.Asp771His } \\
\text { p.Val779Met } \\
\text { p.Ser807Arg } \\
\text { p.Leu829Pro } \\
\text { p.Gly831Asp } \\
84,86\end{array}$ & WFS186 & $4 p 16.3^{86,91,92}$ \\
\hline
\end{tabular}




\begin{tabular}{|c|c|c|c|c|c|}
\hline & & $\begin{array}{l}\text { 1 } \text { Japanese }^{90} \\
1 \text { USA }^{89}\end{array}$ & $\begin{array}{l}\text { p.Ala844Thr' } \\
\text { p.Arg859Pro }\end{array}$ & & \\
\hline DFNA7 & Lacking clinical data & 1 Norwegian ${ }^{93}$ & - & - & $1 \mathrm{q} 21-23^{93}$ \\
\hline DFNA8/12 & $\begin{array}{l}\text { Govaerts 1998, BE }{ }^{94^{\star}} \\
\text { Iwasaki 200295 } \\
\text { Plantinga } 2006^{96}\end{array}$ & 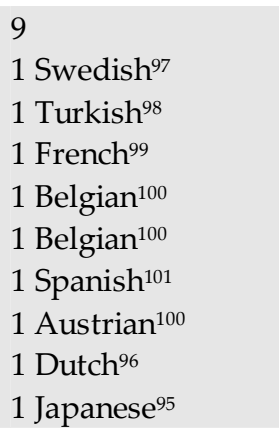 & $\begin{array}{l}\text { p.Cys1057Ser }{ }^{97} \\
\text { p.Cys1509Gly } \\
\text { p.Cys1619Ser } \\
\text { p.Leu1820Phe } \\
\text { p.Gly1824Asp } \\
\text { p. } 100 \\
\text { p.Cys1837Gly } \\
\text { p.Tyr1870 }{ }^{101} \\
\text { p.Arg1890 } \text { Cys }^{96} \\
\text { p.Arg2021His }\end{array}$ & TECTA $^{101}$ & $\begin{array}{l}11 q 22-24 \\
102,103\end{array}$ \\
\hline DFNA9 & 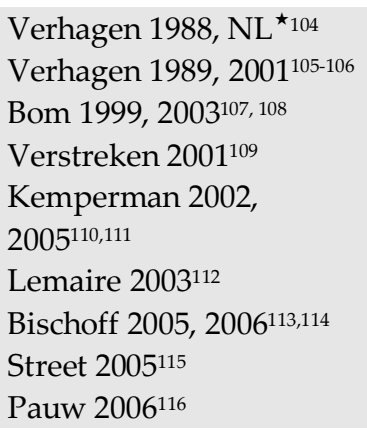 & 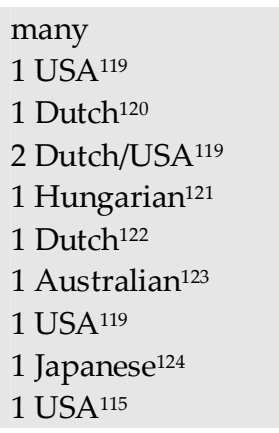 & 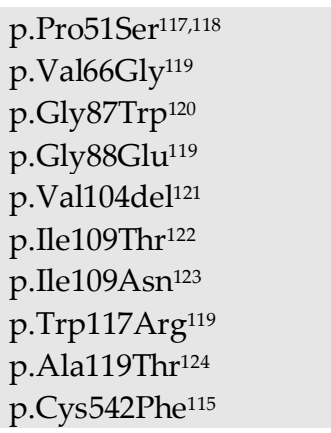 & $\mathrm{COCH}^{119}$ & $14 q 12-13^{125}$ \\
\hline DFNA10 & $\begin{array}{l}\text { Verstreken 2000, BE }{ }^{126^{\star}} \\
\text { De Leenheer 2001, } \\
2002^{127,128}\end{array}$ & $\begin{array}{l}4 \\
1 \text { USA }^{129} \\
\text { 1Belgian }^{129} \\
1 \text { Hungarian }^{130}\end{array}$ & $\begin{array}{l}\text { c. } 1468 \_1469 \text { insAA }^{129} \\
\text { p.Arg587X'129 } \\
\text { c.1558_1559insTTTG }{ }^{130}\end{array}$ & EYA $4^{129}$ & $6 q 22-23^{131}$ \\
\hline DFNA11 & $\begin{array}{l}\text { Tamagawa 1996, JP8* } \\
\text { Tamagawa } 2002^{132} \\
\text { Street 2004 }{ }^{133} \\
\text { Bischoff } 2006^{134}\end{array}$ & $\begin{array}{l}5 \\
1 \text { Italian }{ }^{135} \\
1 \text { Dutch }^{136} \\
1 \text { USA }^{133} \\
1 \text { German }^{137} \\
1 \text { Japanese }\end{array}$ & $\begin{array}{l}\text { p.Ala230Val }{ }^{135} \\
\text { p.Asn458Ile }{ }^{136} \\
\text { p.Gly722Arg } \\
\text { p.Arg853Cys } \\
\text { p.Ala886_Lys } 888 \text { del }^{137}\end{array}$ & $\mathrm{MYO7A}^{9}$ & $11 q 12.3-21^{8}$ \\
\hline DFNA13 & $\begin{array}{l}\text { Kunst 2000, NL }{ }^{138^{\star}} \\
\text { De Leenheer } 2001 \text { 2002, } \\
\text { 2004 }\end{array}$ & $\begin{array}{l}2 \\
1 \text { Dutch }^{142} \\
1 \text { USA }^{142}\end{array}$ & $\begin{array}{l}\text { p.Gly323Glu' }{ }^{142} \\
\text { p.Arg549Cys }\end{array}$ & $\begin{array}{l}\text { COL11A2 } \\
142\end{array}$ & $6 \mathrm{p} 21.3^{143}$ \\
\hline DFNA15 & $\begin{array}{l}\text { Frydman 2000, } \mathrm{IL}^{144^{\star}} \\
\text { Gottfried 2002 }\end{array}$ & 1 Israelian ${ }^{146}$ & c. $880 \_887$ del 146 & POU4F3146 & $5 q 31-33^{146}$ \\
\hline DFNA16 & Lacking clinical data & 1 Japanese ${ }^{147}$ & - & - & $2 q 23-24.3^{147}$ \\
\hline DFNA17 & Lalwani 2002 , USA ${ }^{148 \star}$ & $1 \mathrm{USA}^{149}$ & p.Arg705His ${ }^{149}$ & $\mathrm{MYH}^{149}$ & $22 q 12-13^{150}$ \\
\hline DFNA18 & Lacking clinical data & 1 German $^{151}$ & & - & $3 q 22^{151}$ \\
\hline DFNA19 & - & - & - & - & 10 pcent $^{152}$ \\
\hline DFNA20/26 & 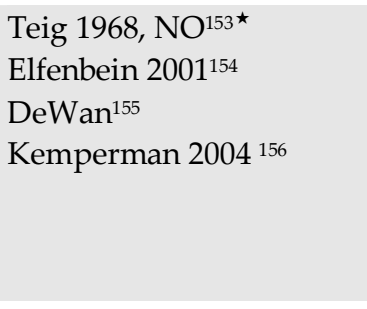 & $\begin{array}{l}6 \\
1 \text { USA }^{157} \\
1 \text { USA }^{157} \\
1 \text { USA }^{157} \\
1 \text { USA }^{157} \\
1 \text { Dutch }^{158} \\
1 \text { Norwegian }^{159}\end{array}$ & $\begin{array}{l}\text { p.Thr89Ile157 } \\
\text { p.Lys118Met } \\
\text { p.Pro332Ala }{ }^{157} \\
\text { p.Pro264Leu } \\
\text { p.Thr278Ile }{ }^{158} \\
\text { p.Val370Ala }{ }^{159}\end{array}$ & ACTG1 157 & $17 \mathrm{q} 25^{160}$ \\
\hline DFNA21 & Kunst 2000, NL ${ }^{161 \star}$ & 1 Dutch $^{161}$ & - & - & 6 p22-24 ${ }^{161,162}$ \\
\hline DFNA22 & Topsakal 2006, BE $163^{\star}$ & $\begin{array}{l}2 \\
1 \text { Italian }^{163}\end{array}$ & p.Cys442Tyr ${ }^{163}$ & MYO66 $^{164}$ & $6 q 13^{164}$ \\
\hline DFNA23음 & Lacking clinical data & 1 Swiss/German ${ }^{165}$ & - & SIX1 $1^{166}$ & $14 q 21-22^{165}$ \\
\hline DFNA24 & Santos 2006, $\mathrm{CH}^{167 \star}$ & 1 Swiss/German 168 & - & - & $4 q 35 \operatorname{ter}^{168}$ \\
\hline
\end{tabular}


Introduction

\begin{tabular}{|c|c|c|c|c|c|}
\hline DFNA25 & Lacking clinical data & $1 \mathrm{USA}^{169}$ & - & - & $12 q 22-24^{169}$ \\
\hline DFNA27 & - & - & - & - & $4 q 12^{170}$ \\
\hline DFNA28 & Lacking clinical data & $1 \mathrm{USA}^{171}$ & c.1609_1610insC ${ }^{171}$ & TFCP2L3171 & $8 \mathrm{q} 22^{171}$ \\
\hline DFNA29 & - & - & - & reserved & - \\
\hline DFNA30" & Lacking clinical data & 1 Italian $^{172}$ & - & - & $15 q 25-26^{172}$ \\
\hline DFNA31 ${ }^{\circ}$ & Ensink 2001, NL ${ }^{173 \star}$ & 1 Dutch $^{174}$ & - & - & $6 \mathrm{p} 21.3^{174}$ \\
\hline DFNA32 & - & - & - & - & 11p15175 \\
\hline DFNA33 & - & - & - & reserved & - \\
\hline DFNA34 & Lacking clinical data & - & - & - & $1 q 44176$ \\
\hline DFNA35 & - & - & - & reserved & - \\
\hline DFNA36 & Makishima 2004, USA $^{177^{\star}}$ & $1 \mathrm{USA}^{178}$ & p.Asp572Asn ${ }^{178}$ & $\mathrm{TMC}^{178}$ & $9 q 13-21^{178}$ \\
\hline DFNA37 & Lacking clinical data & 1 USA $^{179}$ & - & - & $1 \mathrm{p} 21^{179}$ \\
\hline DFNA39+ & Lacking clinical data & 2 Chinese $^{180}$ & & DSPP180 & $4 q 21.3^{180}$ \\
\hline DFNA40 & - & - & - & reserved & $16 \mathrm{p} 12$ \\
\hline DFNA41 & Lacking clinical data & 1 Chinese $^{181}$ & - & - & 12q24-qter 181 \\
\hline DFNA42 & Lacking clinical data & 1 Chinese $^{182}$ & - & - & $4 q 28^{182}$ \\
\hline DFNA43 & Lacking clinical data & 1 Italian $^{183}$ & - & - & $2 \mathrm{p} 12^{183}$ \\
\hline DFNA44 & Lacking clinical data & 1 Spanish $^{184}$ & - & - & $3 q 28-29^{184}$ \\
\hline DFNA45 & - & - & - & reserved & - \\
\hline DFNA46 & - & - & - & reserved & - \\
\hline DFNA47 & Lacking clinical data & 1 Italian $^{185}$ & - & - & $9 p 21-22^{185}$ \\
\hline DFNA48 & Lacking clinical data & $\begin{array}{l}1 \text { Italian } \\
8 \text { isolated persons: } \\
186\end{array}$ & $\begin{array}{l}\text { p.Arg93X186 } \\
\text { p.Ser116_Tyr117insSer }{ }^{186} \\
\text { p.Val306Met }{ }^{186} \otimes \\
\text { p.Glu385Asp }{ }^{186} \\
\text { p.Gly662Glu }{ }^{186} \otimes \\
\text { p.Gly674Asp }{ }^{186} \otimes \\
\text { p.Ser797Phe }{ }^{186} \\
\text { p.Ser910Pro }{ }^{186} \otimes\end{array}$ & MYO1A $^{186}$ & $12 q 13-14^{187}$ \\
\hline DFNA49 & Lacking clinical data & 1 Spanish $^{188}$ & - & - & $1 q 21-23^{188}$ \\
\hline DFNA50 & Lacking clinical data & 1 Spanish $^{189}$ & - & - & $7 q 32^{189}$ \\
\hline DFNA51 & - & - & - & reserved & $9 q 21$ \\
\hline DFNA52 & - & - & - & reserved & $4 q 28$ \\
\hline DFNA53 & Lacking clinical data & 1 Chinese $^{190}$ & - & - & $14 q 11-12^{190}$ \\
\hline DFNA54œ & Lacking clinical data & 1 Swiss $^{191}$ & - & - & $5 q 31^{191}$ \\
\hline
\end{tabular}

X: stopcodon, del; deletion, ins; insertion; pcent; pericentral, c.; changes at the coding DNA level, p.; changes at the protein level; gr.; group. ${ }^{*}$ At first the number of all families is reported, below this, the number and origin of the families with a known mutation are reported; ${ }^{\star}$ Indicates first clinical description together with the country abbreviation; $\wedge$ This mutation is believed not to be a disease causing mutation, but a polymorphism; 을 - One patient with solitary left hypoplastic kidney with vesicoureteral reflux and progressive renal failure, suggesting BOR syndrome ${ }^{166}$; ${ }^{D F N A 30}$ shows overlap with OTSC1, a locus for otosclerosis, but patients do not have signs of otosclerosis ${ }^{171} ; \unrhd$ This family was first linked to DFNA13; † Not a non-syndromic form of hearing impairment, but dentinogenesis imperfecta, associated with hearing impairment in some families; $\otimes$ No family records available, no certainty of autosomal dominant inheritance; $\infty$ Shows overlap with DFNA15 but with different phenotype. 


\section{DFNA5}

\section{Clinical characteristics}

At present, three different DFNA5 families have been described. The first family in which the locus was identified was of Dutch origin and showed progressive symmetrical hearing loss that started at the high frequencies. ${ }^{72,73,192-195}$ Later, another Dutch family and a Chinese family were encountered. ${ }^{75,77}$ The audiograms had so-called Z-shaped profiles. ${ }^{74,75}$ Clinical data on the two Dutch families demonstrated similar pure-tone hearing thresholds with more favourable speech recognition in the second family, owing to better speech frequency thresholds in the first four decades (annual threshold deterioration (ATD) of 1 to 4 versus 1.2 to $1.4 \mathrm{~dB}$ per year at 2 to $8 \mathrm{kHz}$ ). Vestibular function was normal in the two Dutch families. Unfortunately, it was not possible to make comparisons with the phenotype of the Chinese family because of the lack of clinical data in the Chinese report. Table 2 lists the clinical and genetic data of the three DFNA5 families.

Table 2. Phenotype and genotype of DFNA5

\begin{tabular}{|l|l|l|l|l|l|l|}
\hline Origin & Site of Mutation & $\begin{array}{l}\text { Effect of } \\
\text { Mutation }\end{array}$ & $\begin{array}{l}\text { Hearing } \\
\text { Onset }\end{array}$ & $\begin{array}{l}\text { Hearing } \\
\text { Impairment }\end{array}$ & $\begin{array}{l}\text { Vestibular Dysfunction } \\
\text { Subjective }\end{array}$ \\
\hline Netherlands & $\begin{array}{l}\text { IVS7, } \\
\text { Objective }\end{array}$ \\
\hline Netherlands & IVS7-6 C>G & Exon 8 del & $\begin{array}{l}5-15 \mathrm{y} \\
\text { high frequency }\end{array}$ & absent & absent \\
\hline China & IVS7-20-22del CTT & Exon 8 del & $7-30 \mathrm{y}$ & high frequency & $\begin{array}{l}\text { not } \\
\text { Exonallable }\end{array}$ & not available \\
\hline
\end{tabular}

IVS, intervening sequence (intron); bp, base pair; del, deletion, ins, insertion, y, years.

\section{Genetic aspects}

DFNA5 was mapped to chromosome 7 p15 in $1995 .^{78}$ By positional cloning, a complex insertion/deletion mutation was found in intron 7 that caused skipping of exon 8 in the messenger RNA (mRNA). This leads to premature termination of the encoded protein. ${ }^{76}$ In the second Dutch DFNA5 family, a mutation was present in the splice acceptor site of intron 7 that caused skipping of exon 8 in part of the mRNA. ${ }^{75}$ Furthermore, another mutation in the Chinese DFNA5 family with deletion in intron 7 also caused skipping of exon 8 in the mRNA. ${ }^{77}$ All mutations have the same effect on the mRNA and the protein. Therefore, it was hypothesized that the effect of skipping of exon 8 is crucial in the development of hearing impairment in DFNA5.

At present, little is known about the function of the DFNA5 protein. It is expressed in the cochlea and placenta and at low levels in the heart, brain, kidney, 
lung, liver, pancreas and skeletal muscle. ${ }^{76,196}$ In the mouse, Dfna5 expression is present in the developing and mature cochlea. ${ }^{197}$

Van Laer et al. generated Dfna5 knockout mice that did not show cochleovestibular impairment. ${ }^{1}$ Detailed analysis of the cochlea revealed that even at birth, there were significant differences in the number of fourth row outer hair cells between the knockout mice and the wild-type mice. In view of the absence of hearing loss in the knockout mice, it was hypothesized that DFNA5-related hearing impairment in humans is caused by a gain of function. This was supported by the toxic effect of mutant DFNA5 on yeast cells and mammalian cells and their loss of viability. ${ }^{1,2,198}$

Dunø et al. also suggested that DFNA5 mutations caused a gain of function, because two cases with deletion in the 7p14-p15 locus that included DFNA5 had normal hearing. ${ }^{199}$

Examination of Dfna5 knockdown zebrafish revealed malformation of the semicircular canals and pharyngeal cartilage. The Dfna5 knockdown phenotype resembled that of the Jekyll mutant, with a mutated $u g d h$ gene. It also showed loss of ugdh expression and low hyaluronic acid levels. ${ }^{200}$ Hyaluronic acid was considered to be important in the differentiation of cartilage and the projection outgrowth of the protrusions of the semicircular canals in the embryonic ear. ${ }^{201}$ Reductions in hyaluronic acid may lead to uncoordinated growth and collapse of the protrusions, with destruction of the semicircular canals. The DFNA5 mutation is not a null mutation. Therefore, it is difficult to suggest a correlation between the zebrafish phenotype and DFNA5.

Pejvakin, a protein encoded by DFNB59, is a member of the DFNA5DC protein family that also includes DFNA5. ${ }^{202,203}$ DFNB59 causes non-syndromic, prelingual, severe to profound hearing impairment with auditory neuropathy and normal cochlear hair cell function. As DFNA5 and pejvakin occupy homologous regions it has been suggested that they are paralogs. ${ }^{203}$ The function of pejvakin is unknown, so the homology between the proteins does not provide any clues about DFNA5 function.

It has been hypothesized that DFNA5 is involved in different forms of cell death, apoptosis and necrosis during the development of the ear, or later in life. 1,198 DFNA5 is also believed to play a role in tumour biology. ${ }^{204,205}$ 


\section{DFNA9}

\section{Clinical characteristics}

Audiometry

DFNA9 causes autosomal dominant progressive sensorineural hearing impairment, predominantly at the high frequencies, with progressive vestibular dysfunction. ${ }^{104-113,116,119,122,123,206-210}$ The first clinical description comprised a Dutch DFNA9 family, before gene linkage studies had been performed for nonsyndromic autosomal dominant types of hearing loss. ${ }^{104}$ Ten different mutations have been identified in DFNA9 in the $\mathrm{COCH}$ (coagulation factor $\underline{\mathrm{C}}$ homology) gene: Val66Gly, Gly88Glu, Trp117Arg, ${ }^{119}$ Pro51Ser, ${ }^{117,118}$ Ile109Asn, ${ }^{123}$ Ala119Thr, ${ }^{124}$ Val104del, ${ }^{121}$ Cys542Phe, ${ }^{115}$ Gly87Trp ${ }^{120}$ and Ile109Thr ${ }^{122}$ (Table 3). Many families in the Netherlands and Belgium are known to have the Pro51Ser founder mutation ${ }^{211}$ and two families are known to have the Gly88Glu mutation. ${ }^{111,119}$ All the other DFNA9 mutations have been identified in just one family. Hearing impairment started in the second to third decades in the American families with the Val66Gly and Trp117Arg mutations. ${ }^{119,209}$ In contrast, it developed from the fourth to fifth decade onwards in all the other DFNA9 families. ${ }^{111,116-119,121-124}$ The age of onset in the family with the Cys542Phe mutation has not yet been determined. ${ }^{115}$ ATD values were around 2 to $7 \mathrm{~dB}$, which eventually led to (sub)residual hearing threshold levels. ${ }^{107,110,111,113,117}$ Speech recognition was fairly poor, deteriorated rapidly and resembled presbyacusis, but at a younger age. ${ }^{116}$

A high prevalence of vascular disease was found in two families with the Pro51Ser mutation. ${ }^{105,107}$ Although it has been suggested that DFNA9 causes strangulation of cochlear nerves and degeneration of hair cells, cochlear implantation appeared to be successful in DFNA9 patients with severe to profound hearing loss..$^{212-214}$

\section{Vestibular function}

The vestibular dysfunction that is associated with DFNA9 is progressive and its expression varies between families with different mutations. In the families with the Pro51Ser and Ile109Asn mutations, the vestibular phenotype was fully penetrant. ${ }^{113,123}$ In the family harbouring the Gly87Trp substitution and the two families with the Gly88Glu mutation, expression was 89(\%) and 40-50(\%), respectively. ${ }^{111,116,119}$ Expression was also incomplete in the families harbouring the Val66Gly, Trp117Arg and Ile109Thr mutations, but it was not clear in the family with the Ala119Thr mutation. ${ }^{122,124,209,215}$ In the family with the Val104del mutation, vestibular symptoms were only reported in the proband, who had vestibular areflexia. ${ }^{121}$ No subjective vestibular dysfunction was described in the first four 
decades of life in the family that carried the Cys542Phe substitution. ${ }^{115}$ Vestibular tests showed dysfunction of the horizontal semicircular canal and probably the saccular otolith in two of three persons. ${ }^{115}$

In families with the Pro51Ser mutation vestibular impairment started at an earlier age and showed more rapid progression. Ultimately, loss of function was more complete than the hearing impairment. ${ }^{113}$ In the Dutch families that carry the Gly88Glu and Gly87Trp mutations, the frequency of vestibular areflexia was significantly lower and the age of onset of vestibular impairment seemed to be higher than in the families with the Pro51Ser substitution. Age of onset of hearing impairment was similar in all families harbouring the three mutations. ${ }^{111,116}$ The age of onset of vestibular impairment in the family with the Val66Gly mutation was extremely variable and the symptoms started a few years later than the hearing impairment. ${ }^{215}$

Ménière-like vertiginous attacks have been reported in families that carry the Pro51Ser founder mutation and also in other DFNA9 families. ${ }^{106,109,112,118,123,124,208}$ However, unlike Ménière's disease, DFNA9 causes non-fluctuating highfrequency hearing loss, without any signs of endolymphatic hydrops. In addition, $\mathrm{COCH}$ mutations were not found in patients with Ménière's disease. ${ }^{124,216}$ Consequently, the association appears to be epiphenomenal. ${ }^{215}$

\section{Vertical corneal striae}

Recently, subclinical vertical corneal striae have been found in three DFNA9 families, two of whom were carrying the Pro51Ser mutation and one the Gly88Glu mutation. ${ }^{114}$ No vertical corneal striae were present in the general Dutch ophthalmologic population or in a fourth family with the Gly87Trp mutation, except in one family member who had keratoconus. The striae were observed from the age of 47 years onwards in 32 individuals, of whom 27 individuals had a $\mathrm{COCH}$ mutation. Most frequent symptoms were dry and injected eyes and instability of refraction and vision. We found a significant association between the corneal striae and the midlife-onset cochleovestibular dysfunction in the three DFNA9 families, which suggests that these two features are caused by Pro51Ser and Gly88Glu COCH mutations. ${ }^{114}$ A sequence variant in a neighbouring gene might also cause the cosegregation of the corneal phenotype with the DFNA9 mutations. However, it is unlikely because the striae were present in three DFNA9 families with two different $\mathrm{COCH}$ mutations and this phenomenon has not been described previously. The suggestion that $\mathrm{COCH}$ gene mutations caused the vertical corneal striae was supported by the identification of co-deposits of cochlin and acidophilic mucopolysaccharide in the trabecular meshwork of patients with 
primary open angle glaucoma and in glaucomatous DBA/2J mice with progressive hearing loss, which were absent in age-matched control donor eyes. ${ }^{27,218}$ This theory was also sustained by the detection of $\mathrm{COCH}$ gene transcripts in the human cornea using RT-PCR ${ }^{114}$ and in a cDNA library constructed using RNA from the anterior segment of the zebrafish eye. ${ }^{219}$

Table 3. Phenotype and genotype of DFNA9

\begin{tabular}{|c|c|c|c|c|c|c|c|c|}
\hline Origin & Exon & $\begin{array}{l}\text { Amino } \\
\text { Acid } \\
\text { Change }\end{array}$ & $\begin{array}{l}\text { Affected } \\
\text { Domain }\end{array}$ & $\begin{array}{l}\text { Hearing } \\
\text { Onset }\end{array}$ & $\begin{array}{l}\text { Hearing } \\
\text { Impairment }\end{array}$ & \multicolumn{2}{|c|}{$\begin{array}{l}\text { Vestibular Dysfunction } \\
\text { Subjective Objective }\end{array}$} & $\begin{array}{l}\text { Corneal } \\
\text { Involvement }\end{array}$ \\
\hline Netherlands & 4 & Pro51Ser & LCCL & $40 \mathrm{y}$ & $\begin{array}{l}\text { high } \\
\text { frequency }\end{array}$ & $100 \%$ & $100 \%$ & 2 families \\
\hline USA & 4 & Val66Gly & LCCL & $\begin{array}{l}2^{\text {nd }}-3^{\text {rd }} \\
\text { decade }\end{array}$ & $\begin{array}{l}\text { high } \\
\text { frequency }\end{array}$ & 1 out of 3 & 3 out of 3 & not available \\
\hline Netherlands & 5 & Gly87Trp & LCCL & $43 y$ & $\begin{array}{l}\text { high } \\
\text { frequency }\end{array}$ & $\begin{array}{l}15 \text { out of } \\
28\end{array}$ & 10 out of 12 & absent \\
\hline $\begin{array}{l}\text { USA/ } \\
\text { Netherlands }\end{array}$ & 5 & Gly88Glu & LCCL & $46-49 y$ & $\begin{array}{l}\text { high } \\
\text { frequency }\end{array}$ & $40(\%)^{* *}$ & $40 \% * *$ & 1 family \\
\hline Hungary & 5 & Val104del & LCCL & $32 y^{*}$ & $\begin{array}{l}\text { high } \\
\text { frequency }\end{array}$ & 1 out of 1 & areflexia* & not available \\
\hline Netherlands & 5 & Ile109Thr & LCCL & $43 y$ & $\begin{array}{l}\text { high } \\
\text { frequency }\end{array}$ & 5 out of 11 & 4 out of 7 & absent \\
\hline Australia & 5 & Ile109Asn & LCCL & $\begin{array}{l}4^{\text {th }}-5^{\text {th }} \\
\text { decade }^{\circ}\end{array}$ & $\begin{array}{l}\text { high } \\
\text { frequency }\end{array}$ & $100 \%$ & $100 \%$ & not available \\
\hline USA & 5 & Trp117Arg & LCCL & $\begin{array}{l}\text { early } 3^{\text {rd }} \\
\text { decade }\end{array}$ & $\begin{array}{l}\text { high } \\
\text { frequency }\end{array}$ & some & $?$ & not available \\
\hline Japan & 5 & Ala119Thr & LCCL & $30-35 y$ & $\begin{array}{l}\text { high } \\
\text { frequency }\end{array}$ & $\begin{array}{l}\text { not } \\
\text { available }\end{array}$ & $\begin{array}{l}\text { not } \\
\text { available }\end{array}$ & not available \\
\hline USA & 12 & Cys542Phe & vWFA 2 & $\begin{array}{l}\text { not } \\
\text { available }\end{array}$ & $\begin{array}{l}\text { high } \\
\text { frequency }\end{array}$ & absent & $\begin{array}{l}2 \text { out of } 3, \\
\text { no areflexia }\end{array}$ & not available \\
\hline
\end{tabular}

$\mathrm{y}$, years; ${ }^{*} 1$ patient available; ${ }^{* *}$ Data derived from the Dutch Gly88Glu family, ${ }^{\circ}$ in erratum corrected ${ }^{123}$

\section{Genetic aspects}

In 1996, DFNA9 was found to be linked to chromosome 14q12-13.125 The $\mathrm{COCH}$ gene was isolated by using a human foetal cochlear cDNA library with high expression in the cochlear and vestibular labyrinth. ${ }^{220,221} \mathrm{COCH}$ has 12 exons that encode an extracellular matrix protein, called cochlin.222 Cochlin is a stable component of the inner ear and is highly conserved between species. ${ }^{220,221}$ This protein contains a signal peptide, an LCCL domain and two von Willebrand factor A-like domains (vWFA1 and vWFA2). The two domains are involved in haemostasis, the complement and immune system, and the extracellular matrix assembly. ${ }^{223}$ The LCCL domain refers to the three proteins that contain the module $\underline{L}$ imulus factor $\underline{\mathrm{C}}$ clotting protein, $\underline{\text { cochlin }}$ and late gestation lung protein Lgl1. Its 
module is an autonomous folding domain, present in various extracellular modular proteins. ${ }^{224}$ As Factor $C$ is an endotoxin-sensitive serine protease that operates in the Limulus defence system, the LCCL domain plays an important role in antibody-independent host defence. ${ }^{225}$ The expression of Lgl1 in foetal lungs coincides with the production of pulmonary surfactant and might assist in its antimicrobial activity. ${ }^{224,226}$

Liepinsh et al. demonstrated that all the then known mutations (Pro51Ser, Val66Gly, Gly88Glu, Ile109Asn, Trp117Arg) occur in the LCCL domain. With the exception of Trp117Arg, all mutations in the LCCL domain tested disrupt its normal structure and lead to protein misfolding of this domain. ${ }^{226}$ The mutation that causes the Cys542Phe substitution however, is located in the vWFA2 domain. ${ }^{115}$ Cochlin is expressed in the cochlear fibrocytes of the spiral ligament, spiral limbus and osseous spiral lamina and in the fibrocytes of the stroma that underlie the sensory epithelium of the crista ampullaris of the semicircular canals. $^{222,227}$ Cochlin is secreted via the endoplasmatic reticulum and golgi apparatus. It is then cleaved and glycosylated. ${ }^{228}$ Cochlin expression is exclusive to the mesodermal tissues and it is the most abundant protein in the bovine, mouse and human cochlea. 227,229

Results of reverse transcriptase polymerase chain reaction (RT-PCR) on RNA from EBV-transformed cell lines imply that the expression of mutant $\mathrm{COCH}$ transcripts is stable, without degradation in patients with the Pro51Ser mutation. ${ }^{227}$ As no premature termination or truncation is caused by the known $\mathrm{COCH}$ mutations, these findings support the hypothesis of a dominant negative effect in DFNA9, rather than haploinsufficiency. ${ }^{227}$ The presence of cochlin-containing deposits in the inner ear of DFNA9 patients (see next paragraph) and the absence of any obvious pathology in Coch (-/-) mice at five months of age further disputes the concept of haploinsufficiency and confirms that the cochleovestibular dysfunction in DFNA9 is the result of toxic effects by a gain of function mechanism. ${ }^{227,230}$

\section{Histopathology of the inner ear}

The first temporal bone studies of two American DFNA9 families were reported in 1991. ${ }^{209}$ Cellular degeneration of fibrocytes and replacement by homogeneous glycosaminoglycan deposits was found predominantly in the more medial parts of the spiral ligament, the region of the insertion of the spiral limbus into the basilar membrane and in the stroma of the macula and crista in the vestibular labyrinth. Families with Val66Gly, Gly88Glu, Trp117Arg and Pro51Ser mutations showed these anomalies in the temporal bones, which suggests that all the mutations cause the same destruction due to similar pathologic 
processes. ${ }^{209,212,227,231}$ In the labyrinth, the ampullary stroma and wall showed distortion, collapse and thickening. ${ }^{27}$ These inner ear findings are exclusive for DFNA9. Electron microscopic studies revealed that these acidophilic deposits consist of highly branched, non-banded microfibrils, that are believed to lead to disruption of the extracellular matrix assembly. Khetarpal suggested that pathology was secondary to altered interactions between the type A von Willebrand factor domain of cochlin and the surrounding collagens. ${ }^{215}$ Recently it has been found that these deposits do indeed contain cochlin. ${ }^{227}$

Immunohistochemistry on post-mortem tissue from DFNA9 patients with anticochlin antibodies demonstrated staining principally in the areas of the atrophied fibrocytes of the spiral ligament, spiral limbus, spiral lamina, stroma of the crista ampullaris and the ampullary wall. The perivascular areas in the modiolus were also immunopositive for cochlin. Other observations were neuroepithelial and neuronal degeneration in the inner ear. These secondary changes are caused by degradation of the extracellular matrix, or strangulation, or interruption of the blood supply by the accumulation of the mutant cochlin aggregates. ${ }^{215,227}$ Cochlin deposits may aggregate in time and result in late onset progressive cochleovestibular dysfunction. ${ }^{228}$

\section{The function of Cochlin}

Cochlin is believed to have a structural function in the extracellular matrix assembly. Its von Willebrand type A domains may interact with other components of the extracellular matrix assembly, which is crucial to the highly structured architecture. ${ }^{119,221}$

In autoimmune sensorineural hearing loss, cochlin was found to be a key target antigen in immunoglobulin and T-cell mediated mechanisms. Elevated serum levels of anti-cochlin antibodies have been detected in patients with autoimmune sensorineural hearing loss. ${ }^{227,232}$ In the autoimmune-prone SWXJ mice, experimentally induced CD4+ T-cell mediated response to cochlin as a target antigen was reported. The mice became hearing impaired five weeks after immunisation. ${ }^{233,234}$ Inner ear inflammation occurred at the sites with high cochlin expression and the histopathological features in DFNA9. ${ }^{234}$ Furthermore, patients with autoimmune sensorineural hearing loss had significantly higher numbers of circulating $\mathrm{T}$ cells and higher serum cochlin-specific antibody titers than unaffected age-matched controls. ${ }^{235}$

Cochlin may have a role in ion homeostasis. The spiral ligament, spiral lamina and limbus are associated with $\mathrm{K}^{+}$recycling from the hair cells to the endolymph, through a network of gap-junctions. The cochlin deposits present at these sites in 
DFNA9 may cause obstruction of the $\mathrm{K}^{+}$pathways that results in disturbance of ion homeostasis in the inner ear. This condition may be responsible for the cochleovestibular phenotype. ${ }^{118}$

Identification of cochlin in the perivascular areas in the modiolus and the cochlear duct in combination with the high prevalence of cardiovascular disease in families with the Pro51Ser mutation, ${ }^{105,107}$ indicates a possible role of cochlin in blood vessels. This hypothesis was corroborated by the presence of von Willebrand type A domains in cochlin, which are important in haemostasis. ${ }^{224}$

More studies are needed to provide supporting evidence of the different functions of cochlin. In the future, a DFNA9 mouse model will further clarify the long-term changes in the inner ear and eye. This model is currently being evaluated by Robertson et al. ${ }^{227}$

\section{DFNA11}

\section{Clinical characteristics}

DFNA11 causes symmetrical progressive sensorineural hearing impairment at all frequencies and vestibular dysfunction. Five DFNA11 mutations have been described (Table 5): Ala886_Lys888del, ${ }^{9}$ Asn458Ile, ${ }^{136}$ Gly722Arg, ${ }^{133}$ Arg853Cys ${ }^{137}$ and Ala230Val. ${ }^{135}$ The age of onset of DFNA11 varies from early childhood (mostly) to the third decade. Audiograms can have a flat or gently downsloping or sometimes even an ascending shape. The mean ATD is $0.56 \mathrm{~dB}$ per year ${ }^{132}$ or varies between 0.3 and $0.9 \mathrm{~dB}$ per year, depending on the frequency ${ }^{134}$ and hearing impairment becomes moderate to severe. Speech recognition is well preserved in the families with the Asn458Ile and Ala886_Lys888del mutations, but it has not been evaluated in the other families. In contrast with the recessive phenotype DFNB2 and Usher 1B, hearing impairment in DFNA11 families is initially mild and shows only slow progression. The cochleovestibular phenotype was comparable in all the DFNA11 families, except for two. Family members with the Gly722Arg mutation did not have any vestibular problems ${ }^{133}$, while the family with the Ala230Val substitution had more profound hearing impairment and all three patients investigated had vestibular areflexia; the youngest patient was 11 years old. ${ }^{135}$ The clinical and genetic data are listed in Table 4.

DFNA9 and DFNA11 are the only types of autosomal dominant hearing impairment known to be associated with vestibular dysfunction. However, Ishiyama et al. described a new audiovestibular syndrome with a dominant pattern of inheritance, but no mutations in the COCH or MYO7A gene. ${ }^{236}$ Hearing 
impairment started primarily at the high frequencies in the fourth decade, while the onset of vestibular dysfunction occurred early in the seventh decade.

Subclinical retinal dysfunction was present exclusively in the DFNA11 family with the Asn458Ile mutation. Abnormalities were found using Goldmann perimetry, electro-oculography and electroretinography. These findings suggested subtle defects in the retinal pigment epithelial cells and photoreceptor cells, in which myosin VIIa was expressed. ${ }^{134}$

Table 4. Phenotype and genotype of DFNA11

\begin{tabular}{|c|c|c|c|c|c|c|c|c|}
\hline Origin & Exon & $\begin{array}{l}\text { Amino } \\
\text { Acid } \\
\text { Change }\end{array}$ & $\begin{array}{l}\text { Affected } \\
\text { Domain }\end{array}$ & $\begin{array}{l}\text { Hearing } \\
\text { Onset }\end{array}$ & Hearing Impairment & $\begin{array}{l}\text { Vestibular } \\
\text { Subjective }\end{array}$ & $\begin{array}{l}\text { Oysfunction } \\
\text { Objective }\end{array}$ & $\begin{array}{l}\text { Retinal } \\
\text { Involvement }\end{array}$ \\
\hline Italy & 7 & Ala230Val & $\begin{array}{l}\text { motor } \\
\text { domain }\end{array}$ & $6 y$ & $\begin{array}{l}\text { Moderate-profound, } \\
\text { flat }\end{array}$ & minor & all areflexia & absent \\
\hline Netherlands & 13 & Asn458Ile & $\begin{array}{l}\text { motor } \\
\text { domain }\end{array}$ & $4-43 y$ & 1.f. $\rightarrow$ flat $\rightarrow$ downsloping & 4 out of 6 & 4 out of 6 & subclinical \\
\hline US & 17 & Gly722Arg & $\begin{array}{l}\text { motor } \\
\text { domain }\end{array}$ & $20-30 y$ & 1.f. $\rightarrow$ flat $\rightarrow$ downsloping & absent & $\begin{array}{l}\text { not } \\
\text { available }\end{array}$ & absent \\
\hline Germany & 21 & Arg853Cys & $\begin{array}{l}\text { IQ5 } \\
\text { domain }\end{array}$ & childhood & not available & $\begin{array}{l}\text { not } \\
\text { available }\end{array}$ & 1 out of 5 & absent \\
\hline Japan & 22 & $\begin{array}{l}\text { Ala886_- } \\
\text { Lys888del }\end{array}$ & $\begin{array}{l}\text { coiled } \\
\text { coil } \\
\text { region }\end{array}$ & $12-16 y$ & flat $\rightarrow$ downsloping & absent & 3 out of 5 & absent \\
\hline
\end{tabular}

1.f., low frequency; $y$, years.

\section{Genetic aspects}

In 1996, DFNA11 was localized to chromosome 11q13.5. ${ }^{8}$ DFNA11 is caused by mutations in $M Y O 7 A$. This gene is also involved in autosomal recessive hearing impairment (DFNB2), ${ }^{10,11}$ Usher syndrome type 1B (USH1B) ${ }^{12}$ and atypical Usher syndrome. ${ }^{13,14}$ Mutations in other unconventional myosin genes are also responsible for different types of hereditary hearing impairment, such as MYO1A in DFNA48, MYO3A in DFNB30, MYO6 in DFNA22 and DFNB37 and MYO15A in DFNB3 (Hereditary Hearing Loss Homepage.) These different interactions demonstrate the important function of myosins in the inner ear. Myosin VIIa consists of a highly conserved motor domain with actin and ATP-binding sites, five IQ regions that are expected to bind calmodulin ${ }^{137,237}$ and a long tail. The tail contains a coiled-coil dimerization domain and two MyTH4 FERM repeats, separated by a poorly conserved SH3 domain. ${ }^{238-241}$ Although the function of the MyTH4 domains is not yet understood, the FERM domains are involved in membrane attachment. ${ }^{240}$ 
Myosin VIIa is expressed exclusively in epithelial tissues, principally those that contain cilia or microvilli, such as the inner ear, retina, testis, lung, kidney, liver, olfactory epithelium and intestine. ${ }^{242-244}$ In the cochlea, myosin VIIa expression is more prominent in inner hair cells than in outer hair cells. Localization is mainly in the stereocilia, but it also occurs in the cell bodies, the cuticular plate, the pericuticular necklace and the synaptic region. ${ }^{239,242,244-246}$ In addition, myosin VIIa is present in type I and type II hair cells of the utricle and semicircular canals. ${ }^{242}$ In the eye, it is highly expressed in the apical microvilli-like processes of retinal pigment epithelial cells and in the connecting cilium and photoreceptor synapses of the photoreceptor cells. ${ }^{244-247}$

Mutations in DFNA11 cause structural changes in either the motor domain or the IQ5 domain and lead to dysfunctional ATP/ADP binding, impairment of the power-stroke or the molecular switch. ${ }^{136,137}$ It has been suggested that MYO7A mutations have a dominant negative effect due to incorrect dimerization in the family with the Ala886_Lys888del mutation, ${ }^{132}$ or due to correct dimerization with wild-type proteins that impair protein function in the families with Asn458Ile and Arg853Cys mutations. ${ }^{136,137}$ Clinical severity varied between two branches of the family described by Street et al., which suggests the presence of a genetic modifier. $^{133}$

\section{The function of myosin VIIa}

Myosin VIIa is a membrane-associated motor molecule that moves along actin filaments by means of actin-activated ATP-ase activity. Owing to its spatiotemporal expression pattern and binding of the tail domain to a number of ligands, ${ }^{248,249}$ it is thought to be involved in several processes, such as the development, differentiation and organization of the cochlear hair cell stereocilia. ${ }^{250-252}$

Part of the Usher syndrome type 1 protein network is formed by myosin VIIa. This protein is present in the developing hair bundles, beneath the cuticular plate of the cochlear and vestibular hair cells and in the synaptic region. ${ }^{249}$ The protein network is important to development, differentiation, and cohesion of the stereocilia during hair cell maturation. ${ }^{251,252}$ In mature hair cells, the Usher protein network may contribute to mechano-electrical transduction processes in the stereocilia ${ }^{241,251-254}$ and it may also be involved in the organization and/or function of cochlear hair cells and retinal photoreceptor synapses. ${ }^{252,255}$

In the eye, myosin VIIa is involved in the migration of melanosomes in the retinal pigment epithelial cells, ${ }^{256-258}$ in the phagocytosis of photoreceptor outer segment tips by retinal pigment epithelial cells ${ }^{259}$ and in the ciliary transport of opsin in 
photoreceptor cells. ${ }^{260}$ The latter is demonstrated by abnormal opsin accumulation in the membrane of the connected cilium in Myo7a-deficient shaker- 1 mice. ${ }^{260}$

The transmembrane protein vezatin is a ubiquitous protein of adherence cell-cell junctions and is attached to cadherin by myosin VIIa. Myosin VIIa is thought to link the actin cytoskeleton to adherence junctions between hair cells and supporting cells to consolidate cell-cell adhesion. The interaction of myosin VIIa with vezatin at the ankle links attachment sites suggests that there is a tension force between the stereocilia that strengthens the cohesion of the hair bundles. ${ }^{241,261}$ In conformity with these notions, shaker-1 mutant mice with a null mutation in Myo7a showed disorganization and disruption of the hair bundles during development and in the end loss of the stereocilia. ${ }^{250,262}$ An autosomal dominant mutation was found in Myo7a in headbanger mice that caused low-frequency hearing loss and vestibular dysfunction. Scanning electron microscopy revealed abnormal stereocilia bundle development from an early age that was more prominent in the apex than in the base of the cochlea. Therefore, this "headbanger gene" was also found to be involved in stereocilia development and preservation. ${ }^{263}$ Further studies on these mouse models are necessary to gain greater understanding of the effects of the different mutations in DFNA11 on the function of myosin VIIa.

\section{Aims of this study}

The core objective of this thesis was to generate new clinical knowledge from a number of families with a non-syndromic autosomal dominant type of hearing loss by performing thorough phenotypic analyses in relation to the genotype in families with DFNA5, DFNA9 and DFNA11. Audiometric and vestibular data were collected from the families to outline and analyse the cochlear and vestibular phenotype. Blood samples were taken for linkage and mutation analyses to identify the genetic defect. Additionally, other clinical aspects were examined, such as speech recognition scores. It also proved worthwhile to include ophthalmologic examinations to obtain data on subclinical features in two of the DFNA types included in this thesis.

Research into hereditary hearing loss has been complicated by the small numbers of patients with each type, the inaccessibility of the inner ear to biopsy and the lack of representative animal models for many types of hereditary hearing loss. Due to the high degree of genetic heterogeneity and the low incidence of the genetic subtypes, diagnostic screening will be too expensive and time-consuming. Therefore, it is important to study phenotype-genotype correlations in order to 
make more specific diagnoses. The major goal is to create phenotypic criteria, such as the ARTA, to enable differentiation between subgroups of non-syndromic hearing impairment. Routine DNA diagnostics are available for the more frequent types of hereditary hearing impairment, such as DFNA9. DNA diagnostics are also available for the MYO7A gene, in the form of the Usher microarray to test known mutations. Knowledge about the different genotypes and the genotypephenotype correlations is important to genetic counselling and provides insight into the molecular biology and function of the inner ear on the basis of the gene product. Greater understanding will contribute to the development of preventive and therapeutic strategies for hearing impairment.

\section{Aims of the study}

1. To evaluate the phenotype and genotype of a DFNA5 family and to compare the phenotypic features to the data on the original DFNA5 family.

2. To evaluate and analyse audiometric and electronystagmographic data obtained from many carriers of the Pro51Ser mutation in the $\mathrm{COCH}$ gene.

3. To describe peculiar vertical corneal striae in families with DFNA9, to test whether there is an association between these vertical corneal striae and $\mathrm{COCH}$ gene mutations and to formulate a plausible causative relation.

4. To outline the cochleovestibular phenotype in a DFNA11 family and to compare the data to the phenotypes of three other DFNA11 families.

5. To describe subclinical retinal abnormalities that are unique to DFNA11.

The results of this study are described in five chapters. Chapter 2 provides clinical and genetic descriptions of a family with DFNA5. Chapter 3 focuses closely on cochleovestibular dysfunction in DFNA9. In Chapter 3.1 an in-depth analysis is reported on many individuals with the Pro51Ser mutation. Chapter 3.2 describes the vertical corneal striae in patients with DFNA9. Chapter 4 reports the cochleovestibular and retinal features in a DFNA11 family. Chapter 5 summarises all the studies and includes the general conclusions and discussion. A Dutch translation of chapter 5 is given in Chapter 6 .

\section{References}

1. Van Laer L, Pfister M, Thys S, Vrijens K, Mueller M, Umans L, Serneels L, Van Nassauw L, Kooy F, Smith RJH, Timmermans JP, Van Leuven F, Van Camp G. Mice lacking Dfna5 show a diverging number of cochlear fourth row outer hair cells. Neurobiol Dis 2005;19:386-399.

2. Gregan J, Van Laer L, Lieto LD, Van Camp G, Kearsey SE. A yeast model for the study of human DFNA5, a gene mutated in nonsyndromic hearing impairment. Biochim Biophys Acta 2003;1638:179-186. 
3. Marazita ML, Ploughman LM, Rawlings B, Remington E, Arnos KS, Nance WE. Genetic epidemiological studies of early-onset deafness in the U.S. school-age population. Am J Med Genet 1993 ;46:486-491.

4. Morton NE. Genetic epidemiology of hearing impairment. Ann N Y Acad Sci 1991;630:16-31.

5. Mehl AL, Thomson V. The Colorado newborn hearing screening project, 1992-1999: on the threshold of effective population-based universal newborn hearing screening. Pediatrics 2002;109:e7.

6. Willems PJ. Genetic causes of hearing loss. N Engl J Med 2000;342:1101-1109.

7. Wang QJ, Lu CY, Li N, Rao SQ, Shi YB, Han DY, Li X, Cao JY, Yu LM, Li QZ, Guan MX, Yang WY, Shen Y. Y-linked inheritance of non-syndromic hearing impairment in a large Chinese family. J Med Genet 2004;41:e80.

8. Tamagawa Y, Kitamura K, Ishida T, Ishikawa K, Tanaka H, Tsuji S, Nishizawa M. A gene for a dominant form of non-syndromic sensorineural deafness (DFNA11) maps within the region containing the DFNB2 recessive deafness gene. Hum Mol Genet 1996;5:849-852.

9. Liu XZ, Walsh J, Tamagawa Y, Kitamura K, Nishizawa M, Steel KP, Brown SD. Autosomal dominant non-syndromic deafness caused by a mutation in myosin VIIA gene. Nat Genet 1997;17:268-269.

10. Liu XZ, Walsh J, Mburu P, Kendrick-Jones J, Cope MJ, Steel KP, Brown SD. Mutations in the myosin VIIA gene cause non-syndromic recessive deafness. Nat Genet 1997;16:188-190.

11. Liu XZ. The clinical presentation of DFNB2. In: Cremers CWRJ, Smith RJH (eds). Genetic hearing impairment. Its clinical presentations. Advances in Oto-Rhino-Laryngol Vol, 61. Basel: Karger 2002;61:120-123.

12. Weil D, Blanchard S, Kaplan J, Guilford P, Gibson F, Walsh J, Mburu P, Varela A, Levilliers J, Weston MD, Kelley PM, Kimberling WJ, Wagenaar M, Levi-Acobas F, Larget-Piet D, Munnich A, Steel KP, Brown SDM, Petit C. Defective myosin VIIA gene responsible for Usher syndrome type 1B. Nature 1995;374:60-61.

13. Liu XZ, Hope C, Walsh J, Newton V, Ke XM, Liang CY, Xu LR, Zhou JM, Trump D, Steel KP, Bundey S, Brown SD. Mutations in the myosin VIIA gene cause a wide phenotypic spectrum, including atypical Usher syndrome. Am J Hum Genet 1998;63:909-912.

14. Ben Zina Z, Masmoudi S, Ayadi H, Chaker F, Ghorbel AM, Drira M, Petit C. From DFNB2 to Usher syndrome: variable expressivity of the same disease. Am J Med Genet 2001;101:181183.

15. Wilde NR. Practical observations on aural surgery and the nature and treatment of diseases of the ear. Philadelphia: Blanchard and Lea. London, Philadelphia: Churchill 1853.

16. Liebreich R. Abkunft aus Ehen unter Blutverwandten als Grund von Retinitis Pigmentosa. Dtsch Arch Klin Med 1861;13:53-55.

17. Hartmann A. Taubstummheit und Taubstummenbildung nach den vorhanden quellen sowie nach eigen Beobachtungen und Erfahrungen. Stuttgart: Verlag Ferdinand Enke, 1880.

18. Politzer A. Lehrbuch der Ohrenheilkunde für praktische Ärzte und Studierenden. Stuttgart: Verlag Ferdinand Enke, 1876 1ste ed, 1887 2te ed.

19. Mendel G. Versuche über Pflantzenhybriden. Verhandlungen des Naturvorschenden Vereins. Brühn, 1865.

20. Albrecht W. Über der Vererbung der hereditären Labyrinthschwerkörigkeit und der Otosclerose. Arch. Ohrenheilk. Nas. Kehlkopfheilk 1922;110:15-48.

21. Kluyskens P, Geldof H. La surdité héréditaire. Acta Oto-Rhino-Laryngologica Belgica 1965;19:1-755.

22. Black FO, Bergstrom L, Downs M, Hemenway W. Congenital deafness. In: A new approach to early detection of deafness through a high risk register. Colorado Associated University Press, Boulder, Colorado 1971. 
23. Cremers CWRJ, Wehberg H. Erfelijke vormen van doofheid geassocieerd met andere symptomen. In Hereditaire aspecten van vroegkinderlijke doofheid. Academisch proefschrift, Nijmegen 1976.

24. Konigsmark BW, Gorlin RJ. Genetic and metabolic deafness. 1976 WB Sanders Company Philadelphia.

25. Fraser GR. The causes of profound deafness in childhood. 1976 The Johns Hopkins University Press, Baltimore.

26. Cremers CWRJ, Hageman MJ, Huizing EH. Erfelijke doofheid en slechthorendheid, Bohn Stafleu en Holkema, Utrecht -Antwerpen 1982.

27. Cremers CWRJ, Fikkers-van Noord M. The earpits-deafness syndrome. Clinical and genetic aspects. Int J Ped Otorhinolaryngol 1980;2:309-322.

28. Brunner HG, van Bennekom A, Lambermon EM, Oei TL, Cremers CWRJ, Wieringa B, Ropers $\mathrm{HH}$. The gene for $\mathrm{X}$-linked progressive mixed deafness with perilymphatic gusher during stapes surgery (DFN3) is linked to PGK. Hum Genet 1988;80:337-340.

29. de Kok YJM, van der Maarel SM, Bitner-Glindzicz M, Huber I, Monaco AP, Malcolm S, Pembrey ME, Ropers HH, Cremers FPM. Association between X-linked mixed deafness and mutations in the POU domain gene POU3F4. Science 1995;267:685-688.

30. Huygen PLM, Pennings RJE, Cremers CWRJ. Characterizing and distinguishing progressive phenotypes in nonsyndromic autosomal dominant hearing impairment. Audiol Med 2003;1:37-46.

31. Huygen PLM, Pauw RJ, Cremers CWRJ. Audiometric profiles associated with genetic nonsyndromic hearing impairment, a review and phenotype analysis. Audiological Medicine. In press.

32. Mazzoli M, Van Camp G, Newton V, Giarbini N, Declau F, Parving A. Recommendations for the description of genetic and audiological data for families with nonsyndromic hereditary hearing impairment. Hereditary Hearing Loss Homepage. Van Camp G, Smith RJH. Hereditary Hearing Loss Homepage. URL: http:// webhost.ua.ac.be/hhh/.

33. León PE, Bonilla JA, Sanchez JR, Vanegas R, Villalobos M, Torres L, Leon F, Howell AL, Rodriguez JA. Low frequency hereditary deafness in man with childhood onset. Am J Hum Genet 1981;33:209-214.

34. Lalwani AK, Jackler RK, Sweetow RW, Lynch ED, Raventos H, Morrow J, King MC, León PE. Further characterization of the DFNA1 audiovestibular phenotype. Arch Otolaryngol Head Neck Surg 1998;124:699-702.

35. Lynch ED, Lee MK, Morrow JE, Welcsh PL, León PE, King MC. Nonsyndromic deafness DFNA1 associated with mutation of a human homolog of the Drosophila gene diaphanous. Science 1997;278:1315-1318.

36. León PE, Raventos H, Lynch E, Morrow J, King MC. The gene for an inherited form of deafness maps to chromosome 5q31. Proc Natl Acad Sci USA Lancet 1992;89:5181-5184.

37. Marres HAM, van Ewijk M, Huygen PLM, Kunst HPM, van Camp G, Coucke PJ, Willems PJ, Cremers CWRJ. Inherited nonsyndromic hearing loss. An audiovestibular study in a large family with autosomal dominant progressive hearing loss related to DFNA2. Arch Otolaryngol Head Neck Surg 1997;123:573-577.

38. Kunst HPM, Marres HAM, Huygen PLM, Ensink RJ, Van Camp G, Van Hauwe P, Coucke PJ, Willems PJ, Cremers CWRJ. Nonsyndromic autosomal dominant progressive sensorineural hearing loss: audiologic analysis of a pedigree linked to DFNA2. Laryngoscope 1998;108:7480.

39. Ensink RJ, Huygen PLM, Van Hauwe P, Coucke PJ, Cremers CWRJ, Van Camp G. A Dutch family with progressive sensorineural hearing impairment linked to the DFNA2 region. Eur Arch Otorhinolaryngol 2000;257:62-67. 
40. Akita J, Abe S, Shinkawa H, Kimberling WJ, Usami S. Clinical and genetic features of nonsyndromic autosomal dominant sensorineural hearing loss: KCNQ4 is a gene responsible in Japanese. J Hum Genet 2001;46:355-361.

41. De Leenheer EMR, Huygen PLM, Coucke PJ, Admiraal RJC, van Camp G, Cremers CWRJ. Longitudinal and cross-sectional phenotype analysis in a new, large Dutch DFNA2/KCNQ4 family. Ann Otol Rhinol Laryngol 2002;111:267-274.

42. De Leenheer EMR, Ensink RJ, Kunst HPM, Marres HAM, Talebizadeh Z, Declau F, Smith SD, Usami S, Van de Heyning PH, Van Camp G, Huygen PLM, Cremers CWRJ. DFNA2/KCNQ4 and its manifestations. In: Cremers CWRJ, Smith RJH (eds). Genetic hearing impairment. Its clinical presentations. Advances in Oto-Rhino-Laryngol Vol, 61. Basel: Karger 2002;61:41-46.

43. Stern RE, Lalwani AK. Audiologic evidence for further genetic heterogeneity at DFNA2. Acta Otolaryngol 2002;122:730-735.

44. Topsakal V, Pennings RJE, te Brinke H, Hamel B, Huygen PLM, Kremer H, Cremers CWRJ. Phenotype determination guides swift genotyping of a DFNA2/KCNQ4 family with a hot spot mutation (W276S). Otol Neurotol 2005;26:52-58.

45. Coucke PJ, Van Hauwe P, Kelley PM, Kunst HPM, Schatteman I, Van Velzen D, Meyers J, Ensink RJ, Verstreken M, Declau F, Marres HAM, Kastury K, Bhasin S, McGuirt WT, Smith RJH, Cremers CWRJ, Van de Heyning PH, Willems PJ, Smith SD, Van Camp G. Mutations in the KCNQ4 gene are responsible for autosomal dominant deafness in four DFNA2 families. Hum Mol Genet 1999;8:1321-1328.

46. Kamada F, Kure S, Kudo T, Suzuki Y, Oshima T, Ichinohe A, Kojima K, Niihori T, Kanno J, Narumi Y, Narisawa A, Kato K, Aoki Y, Ikeda K, Kobayashi T, Matsubara Y. A novel KCNQ4 one-base deletion in a large pedigree with hearing loss: implication for the genotypephenotype correlation. J Hum Genet 2006;51:455-460.

47. Van Hauwe P, Coucke PJ, Ensink RJ, Huygen PLM, Cremers CWRJ, Van Camp G. Mutations in the KCNQ4 K+ channel gene, responsible for autosomal dominant hearing loss, cluster in the channel pore region. Am J Med Genet 2000;93:184-187.

48. Van Camp G, Coucke PJ, Kunst HPM, Schatteman I, Van Velzen D, Marres HAM, van Ewijk M, Declau F, Van Hauwe P, Meyers J, Kenyon J, Smith SD, Smith RJH, Djelantik B, Cremers CWRJ, Van de Heyning PH, Willems PJ. Linkage analysis of progressive hearing loss in five extended families maps the DFNA2 gene to a $1.25-\mathrm{Mb}$ region on chromosome 1p. Genomics 1997;41:70-74.

49. Talebizadeh Z, Kelley PM, Askew JW, Beisel KW, Smith SD. Novel mutation in the KCNQ4 gene in a large kindred with dominant progressive hearing loss. Hum Mutat 1999;14:493-501.

50. Kubisch C, Schroeder BC, Friedrich T, Lutjohann B, El-Amraoui A, Marlin S, Petit C, Jentsch TJ. KCNQ4, a novel potassium channel expressed in sensory outer hair cells, is mutated in dominant deafness. Cell 1999;96:437-446.

51. Coucke PJ, Van Camp G, Djoyodiharjo B, Smith SD, Frants RR, Padberg GW, Darby JK, Huizing EH, Cremers CWRJ, Kimberling WJ, Oostra BA, Van de Heyning PH, Willems PJ. Linkage of autosomal dominant hearing loss to the short arm of chromosome 1 in two families. N Engl J Med 1994;331:425-431.

52. Xia JH, Liu CY, Tang BS, Pan Q, Huang L, Dai HP, Zhang BR, Xie W, Hu DX, Zheng D, Shi XL, Wang DA, Xia K, Yu KP, Liao XD, Feng Y, Yang YF, Xiao JY, Xie DH, Huang JZ. Mutations in the gene encoding gap junction protein beta-3 associated with autosomal dominant hearing impairment. Nat Genet 1998;20:370-373. Erratum in: Nat Genet 1999;21:241.

53. Tekin M, Arnos KS, Xia XJ, Oelrich MK, Liu XZ, Nance WE, Pandya A. W44C mutation in the connexin 26 gene associated with dominant non-syndromic deafness. Clin Genet 2001;59:269273.

54. Denoyelle F, Lina-Granade G, Petit C. DFNA3. In: Cremers CWRJ, Smith RJH (eds). Genetic hearing impairment. Its clinical presentations. Advances in Oto-Rhino-Laryngol Vol, 61. Basel: Karger 2002;61:47-52. 
55. Primignani P, Castorina P, Sironi F, Curcio C, Ambrosetti U, Coviello DA. A novel dominant missense mutation--D179N--in the GJB2 gene (Connexin 26) associated with non-syndromic hearing loss. Clin Genet 2003;63:516-521.

56. Kelsell DP, Dunlop J, Stevens HP, Lench NJ, Liang JN, Parry G, Mueller RF, Leigh IM.Connexin 26 mutations in hereditary non-syndromic sensorineural deafness. Nature 1997;387:80-83.

57. Denoyelle F, Lina-Granade G, Plauchu H, Bruzzone R, Chaib H, Levi-Acobas F, Weil D, Petit C. Connexin 26 gene linked to a dominant deafness. Nature 1998;393:319-320.

58. Melchionda S, Bicego M, Marciano E, Franze A, Morgutti M, Bortone G, Zelante L, Carella M, D'Andrea P. Functional characterization of a novel Cx26 (T55N) mutation associated to nonsyndromic hearing loss. Biochem Biophys Res Commun 2005;337:799-805.

59. Richard G, White TW, Smith LE, Bailey RA, Compton JG, Paul DL, Bale SJ. Functional defects of Cx26 resulting from a heterozygous missense mutation in a family with dominant deafmutism and palmoplantar keratoderma. Hum Genet 1998;103:393-399.

60. Feldmann D, Denoyelle F, Blons H, Lyonnet S, Loundon N, Rouillon I, Hadj-Rabia S, Petit C, Couderc R, Garabedian EN, Marlin S. The GJB2 mutation R75Q can cause nonsyndromic hearing loss DFNA3 or hereditary palmoplantar keratoderma with deafness. Am J Med Genet A 2005 30;137:225-227.

61. Piazza V, Beltramello M, Menniti M, Colao E, Malatesta P, Argento R, Chiarella G, Gallo LV, Catalano M, Perrotti N, Mammano F, Cassandro E. Functional analysis of R75Q mutation in the gene coding for Connexin 26 identified in a family with nonsyndromic hearing loss. Clin Genet 2005;68:161-166.

62. Loffler J, Nekahm D, Hirst-Stadlmann A, Gunther B, Menzel HJ, Utermann G, Janecke AR. Sensorineural hearing loss and the incidence of Cx26 mutations in Austria. Eur J Hum Genet 2001;9:226-230.

63. Xiao ZA, Xie DH. GJB2 (Cx26) gene mutations in Chinese patients with congenital sensorineural deafness and a report of one novel mutation. Chin Med J (Engl). 2004;117:17971801.

64. Morle L, Bozon M, Alloisio N, Latour P, Vandenberghe A, Plauchu H, Collet L, Edery P, Godet J, Lina-Granade G. A novel C202F mutation in the connexin26 gene (GJB2) associated with autosomal dominant isolated hearing loss. J Med Genet 2000;37:368-370.

65. Chaib H, Lina-Granade G, Guilford P, Plauchu H, Levilliers J, Morgon A, Petit C. A gene responsible for a dominant form of neurosensory non-syndromic deafness maps to the NSRD1 recessive deafness gene interval. Hum Mol Genet 1994;3:2219-2222.

66. Grifa A, Wagner CA, D'Ambrosio L, Melchionda S, Bernardi F, Lopez-Bigas N, Rabionet R, Arbones M, Monica MD, Estivill X, Zelante L, Lang F, Gasparini P. Mutations in GJB6 cause nonsyndromic autosomal dominant deafness at DFNA3 locus. Nat Genet. 1999;23:16-18.

67. Kelley PM, Abe S, Askew JW, Smith SD, Usami S, Kimberling WJ. Human connexin 30 (GJB6), a candidate gene for nonsyndromic hearing loss: molecular cloning, tissue-specific expression, and assignment to chromosome 13q12.Genomics 1999;62:172-176.

68. Pusch CM, Meyer B, Kupka S, Smith RJ, Lalwani AK, Zenner HP, Blin N, Nurnberg P, Pfister M. Refinement of the DFNA4 locus to a 1.44 Mb region in 19q13.33. J Mol Med 2004;82:398402.

69. Yang T, Pfister M, Blin N, Zenner HP, Pusch CM, Smith RJ. Genetic heterogeneity of deafness phenotypes linked to DFNA4. Am J Med Genet A 2005;139:9-12.

70. Donaudy F, Snoeckx R, Pfister M, Zenner HP, Blin N, Di Stazio M, Ferrara A, Lanzara C, Ficarella R, Declau F, Pusch CM, Nurnberg P, Melchionda S, Zelante L, Ballana E, Estivill X, Van Camp G, Gasparini P, Savoia A. Nonmuscle myosin heavy-chain gene MYH14 is expressed in cochlea and mutated in patients affected by autosomal dominant hearing impairment (DFNA4). Am J Hum Genet 2004;74:770-776. 
71. Chen AH, Ni L, Fukushima K, Marietta J, O'Neill M, Coucke P, Willems P, Smith RJ. Linkage of a gene for dominant non-syndromic deafness to chromosome 19. Hum Mol Genet 1995;4:1073-1076.

72. Huizing EH, van Bolhuis AH, Odenthal DW. Studies on progressive hereditary perceptive deafness in a family of 335 members. I. Genetical and general audiologic results. Acta Otolaryngol 1966;61:35-41.

73. Huizing EH, van den Wijngaart WSIM, Verschuure J. A follow-up study in a family with dominant progressive inner ear deafness. Acta Otolaryngol 1983;95:620-626.

74. De Leenheer EMR, van Zuijlen DA, Van Laer L, Van Camp G, Huygen PLM, Huizing EH, Cremers CWRJ. Clinical features of DFNA5. In: Cremers CWRJ, Smith RJH (eds). Genetic hearing impairment. Its clinical presentations. Advances in Oto-Rhino-Laryngol Vol, 61. Basel: Karger 2002;61:53-59.

75. Bischoff AMLC, Luijendijk MWJ, Huygen PLM, van Duijnhoven G, De Leenheer EMR, Oudesluijs GG, Van Laer L, Cremers FPM, Cremers CWRJ, Kremer H. A novel mutation identified in the DFNA5 gene in a Dutch family: A clinical and genetic evaluation. Audiol Neurootol 2004;9:34-46.

76. Van Laer L, Huizing EH, Verstreken M, van Zuijlen D, Wauters JG, Bossuyt PJ, Van de Heyning P, McGuirt WT, Smith RJ, Willems PJ, Legan PK, Richardson GP, Van Camp G. Nonsyndromic hearing impairment is associated with a mutation in DFNA5. Nat Genet 1998; 20:194-197.

77. Yu C, Meng X, Zhang S, Zhao G, Hu L, Kong X. A 3-nucleotide deletion in the polypyrimidine tract of the intron 7 of the DFNA5 gene causes nonsyndromic hearing impairment in a Chinese family. Genomics 2003;82: 575-579.

78. Van Camp G, Coucke P, Balemans W, van Velsen D, van de Bilt C, Van Laer L, Smith RJH, Fukushima K, Padberg GW, Frants RR, Van de Heyning P, Smith SD, Huizing EH, Willems PJ. Localisation of a gene for non-syndromic hearing loss (DFNA5) to chromosome 7p15. Hum Mol Genet 1995;4:2159-2163.

79. Vanderbilt University Hereditary Deafness Study Group. Dominantly inherited lowfrequency hearing loss. Arch Otolaryngol 1968;88:242-250.

80. Kunst HPM, Marres HAM, Huygen PLM, Van Camp G, Joosten F, Cremers CWRJ. Autosomal dominant non-syndromal low-frequency sensorineural hearing impairment linked to chromosome 4p16 (DFNA14): statistical analysis of hearing threshold in relation to age and evaluation of vestibulo-ocular functions. Audiology 1999;38:165-173.

81. Bom SJH, Van Camp G, Cryns K, Admiraal RJC, Huygen PLM, Cremers CWRJ. Autosomal dominant low-frequency hearing impairment (DFNA6/14): a clinical and genetic family study. Otol Neurotol 2002;23:876-884.

82. Pennings RJE, Bom SJH, Cryns K, Flothmann K, Huygen PLM, Kremer H, Van Camp G, Cremers CWRJ. Progression of low-frequency sensorineural hearing loss (DFNA6/14-WFS1). Arch Otolaryngol Head Neck Surg 2003;129:421-426.

83. Lesperance MM, Hall JW 3rd, San Agustin TB, Leal SM. Mutations in the Wolfram syndrome type 1 gene (WFS1) define a clinical entity of dominant low-frequency sensorineural hearing loss. Arch Otolaryngol Head Neck Surg 2003;129:411-420.

84. Cryns K, Pfister M, Pennings RJ, Bom SJ, Flothmann K, Caethoven G, Kremer H, Schatteman I, Koln KA, Toth T, Kupka S, Blin N, Nurnberg P, Thiele H, van de Heyning PH, Reardon W, Stephens D, Cremers CWRJ, Smith RJ, Van Camp G. Mutations in the WFS1 gene that cause low-frequency sensorineural hearing loss are small non-inactivating mutations. Hum Genet 2002;110:389-394.

85. Komatsu K, Nakamura N, Ghadami M, Matsumoto N, Kishino T, Ohta T, Niikawa N, Yoshiura K. Confirmation of genetic homogeneity of nonsyndromic low-frequency sensorineural hearing loss by linkage analysis and a DFNA6/14 mutation in a Japanese family. J Hum Genet 2002;47:395-399. 
86. Bespalova IN, Van Camp G, Bom SJ, Brown DJ, Cryns K, DeWan AT, Erson AE, Flothmann K, Kunst HP, Kurnool P, Sivakumaran TA, Cremers CW, Leal SM, Burmeister M, Lesperance MM. Mutations in the Wolfram syndrome 1 gene (WFS1) are a common cause of low frequency sensorineural hearing loss. Hum Mol Genet 2001;10:2501-2508.

87. Kunz J, Marquez-Klaka B, Uebe S, Volz-Peters A, Berger R, Rausch P. Identification of a novel mutation in WFS1 in a family affected by low-frequency hearing impairment. Mutat Res 2003;525:121-124.

88. Young TL, Ives E, Lynch E, Person R, Snook S, MacLaren L, Cater T, Griffin A, Fernandez B, Lee MK, King MC. Non-syndromic progressive hearing loss DFNA38 is caused by heterozygous missense mutation in the Wolfram syndrome gene WFS1. Hum Mol Genet 2001;10:2509-2514.

89. Gürtler N, Kim Y, Mhatre A, Schlegel C, Mathis A, Daniels R, Shelton C, Lalwani AK. Two families with nonsyndromic low-frequency hearing loss harbor novel mutations in Wolfram syndrome gene 1. J Mol Med 2005;83:553-560.

90. Noguchi Y, Yashima T, Hatanaka A, Uzawa M, Yasunami M, Kimura A, Kitamura K. A mutation in Wolfram syndrome type 1 gene in a Japanese family with autosomal dominant low-frequency sensorineural hearing loss. Acta Otolaryngol 2005;125:1189-1194.

91. Lesperance MM, Hall JW 3rd, Bess FH, Fukushima K, Jain PK, Ploplis B, San Agustin TB, Skarka H, Smith RJH, Wills M, Wilcox ER. A gene for autosomal dominant nonsyndromic hereditary hearing impairment maps to 4p16.3. Hum Mol Genet 1995;4:1967-1972.

92. Van Camp G, Kunst H, Flothmann K, McGuirt W, Wauters J, Marres H, Verstreken M, Bespalova IN, Burmeister M, Van de Heyning PH, Smith RJ, Willems PJ, Cremers CW, Lesperance MM. A gene for autosomal dominant hearing impairment (DFNA14) maps to a region on chromosome 4p16.3 that does not overlap the DFNA6 locus. J Med Genet 1999;36:532-536.

93. Fagerheim T, Nilssen O, Raeymaekers P, Brox V, Moum T, Elverland HH, Teig E, Omland $\mathrm{HH}$, Fostad GK, Tranebjaerg L. Identification of a new locus for autosomal dominant nonsyndromic hearing impairment (DFNA7) in a large Norwegian family. Hum Mol Genet 1996;5:1187-1191.

94. Govaerts PJ, De Ceulaer G, Daemers K, Verhoeven K, Van Camp G, Schatteman I, Verstreken $\mathrm{M}$, Willems PJ, Somers T, Offeciers FE. A new autosomal-dominant locus (DFNA12) is responsible for a nonsyndromic, midfrequency, prelingual and nonprogressive sensorineural hearing loss. Am J Otol 1998;19:718-723.

95. Iwasaki S, Harada D, Usami S, Nagura M, Takeshita T, Hoshino T. Association of clinical features with mutation of TECTA in a family with autosomal dominant hearing loss. Arch Otolaryngol Head Neck Surg 2002;128:913-917.

96. Plantinga RF, de Brouwer APM, Huygen PLM, Kunst HPM, Kremer H, Cremers CWRJ. A novel TECTA mutation in a Dutch DFNA8/12 family confirms genotype-phenotype correlation. J Assoc Res Otolaryngol 2006;7:173-181.

97. Balciuniene J, Dahl N, Jalonen P, Verhoeven K, Van Camp G, Borg E, Pettersson U, Jazin EE. Alpha-tectorin involvement in hearing disabilities: one gene--two phenotypes. Hum Genet 1999;105:211-216.

98. Pfister M, Thiele H, Van Camp G, Fransen E, Apaydin F, Aydin O, Leistenschneider P, Devoto M, Zenner HP, Blin N, Nurnberg P, Ozkarakas H, Kupka S. A genotype-phenotype correlation with gender-effect for hearing impairment caused by TECTA mutations. Cell Physiol Biochem 2004;14:369-376.

99. Alloisio N, Morle L, Bozon M, Godet J, Verhoeven K, Van Camp G, Plauchu H, Muller P, Collet L, Lina-Granade G. Mutation in the zonadhesin-like domain of alpha-tectorin associated with autosomal dominant non-syndromic hearing loss. Eur J Hum Genet 1999;7:255-258. 
100. Verhoeven K, Van Laer L, Kirschhofer K, Legan PK, Hughes DC, Schatteman I, Verstreken M, Van Hauwe P, Coucke P, Chen A, Smith RJH, Somers T, Offeciers FE, Van de Heyning PH, Richardson GP, Wachtler F, Kimberling WJ, Willems PJ, Govaerts PJ, Van Camp G. Mutations in the human alpha-tectorin gene cause autosomal dominant non-syndromic hearing impairment. Nat Genet 1998;19:60-62.

101. Moreno Pelayo MA, del Castillo I, Villamar M, Romero L, Hernandez Calvin FJ, Herraiz C, Barbera R, Navas C, Moreno F. A cysteine substitution in the zona pellucida domain of atectorin results in autosomal dominant, postlingual, progressive, mid frequency hearing loss in a Spanish family. J Med Genet 2001;38:E13.

102. Verhoeven K, Van Camp G, Govaerts PJ, Balemans W, Schatteman I, Verstreken M, Van Laer L, Smith RJ, Brown MR, Van de Heyning PH, Somers T, Offeciers FE, Willems PJ. A gene for autosomal dominant nonsyndromic hearing loss (DFNA12) maps to chromosome 11q22-24. Am J Hum Genet 1997;60:1168-1173.

103. Kirschhofer K, Kenyon JB, Hoover DM, Franz P, Weipoltshammer K, Wachtler F, Kimberling WJ. Autosomal-dominant, prelingual, nonprogressive sensorineural hearing loss: localisation of the gene (DFNA8) to chromosome 11q by linkage in an Austrian family. Cytogenet Cell Genet 1998;82:126-130.

104. Verhagen WIM, Huygen PLM, Joosten EMG. Familial progressive vestibulocochlear dysfunction. Arch Neurol 1988;45:766-768.

105. Verhagen WIM, Huygen PLM, Theunissen EJJM, Joosten EMG. Hereditary vestibulo-cochlear dysfunction and vascular disorders. J Neurol Sci 1989;92:55-63.

106. Verhagen WIM, Bom SJH, Fransen E, Van Camp G, Huygen PLM, Theunissen EJJM, Cremers CWRJ. Hereditary cochleovestibular dysfunction due to a $\mathrm{COCH}$ gene mutation (DFNA9): a follow-up study of a family. Clin Otolaryngol 2001;26:477-483.

107. Bom SJH, Kemperman MH, De Kok YJM, Huygen PLM, Verhagen WIM, Cremers FPM, Cremers CWRJ. Progressive cochleovestibular impairment caused by a point mutation in the COCH gene at DFNA9. Laryngoscope 1999;109:1525-1530.

108. Bom SJH, Kemperman MH, Huygen PLM, Luijendijk MWJ, Cremers CWRJ. Cross-sectional analysis of hearing threshold in relation to age in a large family with cochleovestibular impairment thoroughly genotyped for DFNA9/COCH. Ann Otol Rhinol Laryngol 2003;112:280-286.

109. Verstreken M, Declau F, Wuyts FL, D'Haese P, Van Camp G, Fransen E, Van den Hauwe L, Buyle S, Smets REM, Feenstra L, Van der Stappen A, Van de Heyning PH. Hereditary otovestibular dysfunction and Ménière's disease in a large Belgian family is caused by a missense mutation in the COCH gene. Otol Neurotol 2001;22:874-881.

110. Kemperman MH, Bom SJH, Lemaire FX, Verhagen WIM, Huygen PLM, Cremers CWRJ. DFNA9/COCH and its phenotype. In: Cremers CWRJ, Smith RJH (eds). Genetic hearing impairment. Its clinical presentations. Advances in Oto-Rhino-Laryngol Vol, 61. Basel: Karger 2002;61:66-72.

111. Kemperman MH, De Leenheer EMR, Huygen PLM, van Duijnhoven G, Morton CC, Robertson NG, Cremers FPM, Kremer H, Cremers CWRJ. Audiometric, vestibular and genetic aspects of a DFNA9 family with a G88E COCH mutation. Otol Neurotol 2005;26:926933.

112. Lemaire FX, Feenstra L, Huygen PLM, Fransen E, Devriendt K, Van Camp G, Vantrappen G, Cremers CWRJ. Progressive late-onset sensorineural hearing loss and vestibular impairment with vertigo (DFNA9/COCH). Longitudinal analyses in a Belgian family. Otol Neurotol 2003;24:743-748.

113. Bischoff AMLC, Huygen PLM, Kemperman MH, Pennings RJE, Bom SJH, Verhagen WIM, Admiraal RJC, Kremer H, Cremers CWRJ. Vestibular deterioration precedes hearing deterioration in the P51S COCH mutation (DFNA9). An analysis in 74 mutation carriers. Otol Neurotol 2005;26:918-925. 
Introduction

114. Bischoff AMLC, Pauw RJ, Huygen PLM, Aandekerk AL, Kremer H, Cremers CWRJ, Cruysberg JRM. Vertical Corneal Striae in Families with Autosomal Dominant Hearing Loss: DFNA9/COCH. Am J Ophthalmol 2004. In press.

115. Street VA, Kallman JC, Robertson NG, Kuo SF, Morton CC, Phillips JO. A novel DFNA9 mutation in the vWFA2 domain of $\mathrm{COCH}$ alters a conserved cysteine residue and intrachain disulfide bond formation resulting in progressive hearing loss and site-specific vestibular and central oculomotor dysfunction. Am J Med Genet A 2005;139:86-95.

116. Pauw RJ, Collin RWJ, Huygen PLM, Hoefsloot LH, Kremer H, Cremers CWRJ. Clinical characteristics of a Dutch DFNA9 family with a novel $\mathrm{COCH}$ mutation, G87W. Audiol Neurootol 2007;12:77-84.

117. De Kok YJM, Bom SJH, Brunt TM, Kemperman MH, van Beusekom E, van der Velde-Visser SD, Robertson NG, Morton CC, Huygen PLM, Verhagen WIM, Brunner HG, Cremers CWRJ, Cremers FPM. A Pro51Ser mutation in the $\mathrm{COCH}$ gene is associated with late onset autosomal dominant progressive sensorineural hearing loss with vestibular defects. Hum Mol Genet 1999;8:361-366.

118. Fransen E, Verstreken M, Verhagen WIM, Wuyts FL, Huygen PLM, D'Haese P, Robertson NG, Morton CC, McGuirt WT, Smith RJH, Declau F, Van de Heyning PH, Van Camp G. High prevalence of symptoms of Menière's disease in three families with a mutation in the $\mathrm{COCH}$ gene. Hum Mol Genet 1999;8:1425-1429.

119. Robertson NG, Lu L, Heller S, Eavey RD, McKenna M, Nadol JB Jr, Miyamoto RT, Linthicum FH Jr, Lubianca Neto JF, Hudspeth AJ, Seidman CE, Morton CC, Seidman JG. Mutations in a novel cochlear gene cause DFNA9, a human nonsyndromic deafness with vestibular dysfunction. Nat Genet 1998;20:299-303.

120. Collin RW, Pauw RJ, Schoots J, Huygen PL, Hoefsloot LH, Cremers CW, Kremer H. Identification of a novel $\mathrm{COCH}$ mutation, G87W, causing autosomal dominant hearing disorder DFNA9. Am J Med Genet A 2006;140A:1791-1794.

121. Nagy I, Horvath M, Trexler M, Répássy G, Patthy L. A novel COCH mutation, V104del, impairs folding of the LCCL domain of cochlin and causes progressive hearing loss. J Med Genet 2004;41:e9 (http://www.jmedgenet.com/cgi/content/ful/41/1/e9).

122. Pauw RJ, Huygen PLM, Collin RWJ, Cruysberg JRM, Hoefsloot LHS, Kremer H, Cremers CWRJ. Phenotype description of a novel DFNA9/COCH mutation, I109T. Ann Otol Rhinol Laryngol. In press.

123. Kamarinos M, McGill J, Lynch M, Dahl H. Identification of a novel COCH mutation, I109N, highlights the similar clinical features observed in DFNA9 families. Hum Mutat 2001;17:351. Erratum in: Hum Mutat 2001;18:547-548.

124. Usami S, Takahashi K, Yuge I, Ohtsuka A, Namba A, Abe S, Fransen E, Patthy L, Otting G, Van Camp G. Mutations in the $\mathrm{COCH}$ gene are a frequent cause of autosomal dominant progressive cochleo-vestibular dysfunction, but not of Meniere's disease. Eur J Hum Genet 2003;11:744-748.

125. Manolis EN, Yandavi N, Nadol Jr JB, Eavy RD, McKenna M, Rosenbaum S, Khetarpal U, Halpin C, Merchant SN, Duyk GM, MacRae C, Seidman CE, Seidman JG. A gene for nonsyndromic autosomal dominant progressive postlingual sensorineural hearing loss maps to chromosome 14q12-13. Hum Mol Genet 1996; 5:1047-1050.

126. Verstreken M, Declau F, Schatteman I, Van Velzen D, Verhoeven K, Van Camp G, Willems PJ, Kuhweide EW, Verhaert E, D'Haese P, Wuyts FL, Van de Heyning PH. Audiometric analysis of a Belgian family linked to the DFNA10 locus. Am J Otol 2000;21:675-681.

127. De Leenheer EMR, Huygen PLM, Wayne S, Smith RJH, Cremers CWRJ. The DFNA10 phenotype. Ann Otol Rhinol Laryngol 2001;110:861-866.

128. De Leenheer EMR, Huygen PL, Wayne S, Verstreken M, Declau F, Van Camp G, Van de Heyning PH, Smith RJH, Cremers CWRJ. DFNA10/EYA4-The clinical picture. In: Cremers 
CWRJ, Smith RJH (eds). Genetic hearing impairment. Its clinical presentations. Advances in Oto-Rhino-Laryngol Vol, 61. Basel: Karger 2002;61:73-78.

129. Wayne S, Robertson NG, DeClau F, Chen N, Verhoeven K, Prasad S, Tranebjärg L, Morton CC, Ryan AF, Van Camp G, Smith RJ. Mutations in the transcriptional activator EYA4 cause late-onset deafness at the DFNA10 locus. Hum Mol Genet 2001;10:195-200.

130. Pfister M, Toth T, Thiele H, Haack B, Blin N, Zenner HP, Sziklai I, Nurnberg P, Kupka S. A 4bp insertion in the eya-homologous region (eyaHR) of EYA4 causes hearing impairment in a Hungarian family linked to DFNA10. Mol Med 2002;8:607-611.

131. O'Neill ME, Marietta J, Nishimura D, Wayne S, Van Camp G, Van Laer L, Negrini C, Wilcox ER, Chen A, Fukushima K, Ni L, Sheffield VC, Smith RJ. A gene for autosomal dominant lateonset progressive non-syndromic hearing loss, DFNA10, maps to chromosome 6. Hum Mol Genet 1996;5:853-856.

132. Tamagawa Y, Ishikawa K, Ishikawa K, Ishida T, Kitamura K, Makino S, Tsuru T, Ichimura K. Phenotype of DFNA11: a nonsyndromic hearing loss caused by a myosin VIIA mutation. Laryngoscope 2002;112:292-297.

133. Street VA, Kallman JC, Kiemele KL. J Med Genet 2004; Modifier controls severity of a novel dominant low-frequency MyosinVIIA (MYO7A) auditory mutation. J Med Genet 2004; 41:e62.

134. Bischoff AMLC, Pennings RJE, Huygen PLM, Luijendijk MWJ, van Wijk E, Cruysberg JRM, Kremer H, Cremers CWRJ. Cochleovestibular and ocular features in a Dutch DFNA11 family. Otol Neurotol 2006;27:323-331.

135. Di Leva F, D'Adamo P, Cubellis MV, D'Eustacchio A, Errichiello M, Saulino C, Auletta G, Giannini P, Donaudy F, Ciccodicola A, Gasparini P, Franze A, Marciano E. Identification of a Novel Mutation in the Myosin VIIA Motor Domain in a Family with Autosomal Dominant Hearing Loss (DFNA11). Audiol Neurootol. 2006;11:157-164.

136. Luijendijk MWJ, Van Wijk E, Bischoff AMLC, Krieger E, Huygen PLM, Pennings RJE, Brunner HG, Cremers CWRJ, Cremers FPM, Kremer H. Identification and molecular modeling of a mutation in the motor head domain of myosin VIIA in a family with autosomal dominant hearing impairment (DFNA11). Hum Genet 2004;115:149-156.

137. Bolz H, Bolz SS, Schade G, Kothe C, Mohrmann G, Hess M, Gal A. Impaired calmodulin binding of myosin-7A causes autosomal dominant hearing loss (DFNA11). Hum Mutat 2004;24:274-275.

138. Kunst HPM, Huybrechts C, Marres HAM, Huygen PLM, Van Camp G, Cremers CWRJ. The phenotype of DFNA13/COL11A2: nonsyndromic autosomal dominant mid-frequency and high-frequency sensorineural hearing impairment. Am J Otol 2000;21:181-187.

139. De Leenheer EMR, McGuirt WT, Kunst HPM, Huygen PLM, Smith RJH, Cremers CWRJ. The phenotype of DFNA13/COL11A2. In: Cremers CWRJ, Smith RJH (eds). Genetic hearing impairment. Its clinical presentations. Advances in Oto-Rhino-Laryngol Vol, 61. Basel: Karger 2002;61:85-91.

140. De Leenheer EMR, Kunst HPM, McGuirt WT, Prasad SD, Brown MR, Huygen PLM, Smith $\mathrm{RJH}$, Cremers CWRJ. Autosomal dominant inherited hearing impairment caused by a missense mutation in COL11A2 (DFNA13). Arch Otolaryngol Head Neck Surg 2001;127:1317.

141. De Leenheer EMR, Bosman AJ, Kunst HPM, Huygen PLM, Cremers CWRJ. Audiological characteristics of some affected members of a Dutch DFNA13/COL11A2 family. Ann Otol Rhinol Laryngol 2004;113:922-929.

142. McGuirt WT, Prasad SD, Griffith AJ, Kunst HP, Green GE, Shpargel KB, Runge C, Huybrechts C, Mueller RF, Lynch E, King MC, Brunner HG, Cremers CW, Takanosu M, Li SW, Arita M, Mayne R, Prockop DJ, Van Camp G, Smith RJH. Mutations in COL11A2 cause non-syndromic hearing loss (DFNA13). Nat Genet 1999;23:413-419. 
Introduction

143. Brown MR, Tomek MS, Van Laer L, Smith S, Kenyon JB, Van Camp G, Smith RJH. A novel locus for autosomal dominant nonsyndromic hearing loss, DFNA13, maps to chromosome 6p. Am J Hum Genet 1997;61:924-927.

144. Frydman M, Vreugde S, Nageris BI, Weiss S, Vahava O, Avraham KB. Clinical characterization of genetic hearing loss caused by a mutation in the POU4F3 transcription factor. Arch Otolaryngol Head Neck Surg 2000;126:633-637.

145. Gottfried I, Huygen PL, Avraham KB. The clinical presentation of DFNA15/POU4F3. In: Cremers CWRJ, Smith RJH (eds). Genetic hearing impairment. Its clinical presentations. Advances in Oto-Rhino-Laryngol Vol, 61. Basel: Karger 2002;61:92-97.

146. Vahava O, Morell R, Lynch ED, Weiss S, Kagan ME, Ahituv N, Morrow JE, Lee MK, Skvorak AB, Morton CC, Blumenfeld A, Frydman M, Friedman TB, King MC, Avraham KB. Mutation in transcription factor POU4F3 associated with inherited progressive hearing loss in humans. Science 1998;279:1950-1954.

147. Fukushima K, Kasai N, Ueki Y, Nishizaki K, Sugata K, Hirakawa S, Masuda A, Gunduz M, Ninomiya Y, Masuda Y, Sato M, McGuirt WT, Coucke P, Van Camp G, Smith RJH. A gene for fluctuating, progressive autosomal dominant nonsyndromic hearing loss, DFNA16, maps to chromosome 2q23-24.3. Am J Hum Genet 1999;65:141-150.

148. Lalwani AK, Goldstein JA, Mhatre AN. Auditory phenotype of DFNA17. In: Cremers CWRJ, Smith RJH (eds). Genetic hearing impairment. Its clinical presentations. Advances in OtoRhino-Laryngol Vol, 61. Basel: Karger 2002;61:107-112.

149. Lalwani AK, Goldstein JA, Kelley MJ, Luxford W, Castelein CM, Mhatre AN. Human nonsyndromic hereditary deafness DFNA17 is due to a mutation in nonmuscle myosin MYH9. Am J Hum Genet 2000;67:1121-1128.

150. Lalwani AK, Luxford WM, Mhatre AN, Attaie A, Wilcox ER, Castelein CM. A new locus for nonsyndromic hereditary hearing impairment, DFNA17, maps to chromosome 22 and represents a gene for cochleosaccular degeneration. Am J Hum Genet 1999;64:318-323.

151. Bönsch D, Scheer P, Neumann C, Lang-Roth R, Seifert E, Storch P, Weiller C, LamprechtDinnesen A, Deufel T. A novel locus for autosomal dominant, non-syndromic hearing impairment (DFNA18) maps to chromosome 3q22 immediately adjacent to the DM2 locus. Eur J Hum Genet 2001;9:165-170.

152. Green G, Whitehead S, Van Camp G, Smith RJH. 1998. Identification of a new locus DFNA19 - for dominant hearing impairment. The Molecular Biology of Hearing and Deafness meeting, Bethesda, Maryland October:A107.

153. Teig E. Hereditary progressive perceptive deafness in a family of 72 patients. Acta Otolaryng 1968;65: 365-372.

154. Elfenbein JL, Fisher RA, Wei S, Morell RJ, Stewart C, Friedman TB, Friderici K. Audiologic aspects of the search for DFNA20: a gene causing late-onset, progressive, sensorineural hearing loss. Ear Hear 2001;22:279-288.

155. DeWan AT, Parrado AR, Leal SM. A second kindred linked to DFNA20 (17q25.3) reduces the genetic interval. Clin Genet 2003;63:39-45.

156. Kemperman MH, De Leenheer EMR, Huygen PLM, van Wijk E, van Duijnhoven G, Cremers FPM, Kremer H, Cremers CWRJ. A Dutch family with hearing loss linked to the DFNA20/26 locus: longitudinal analysis of hearing impairment. Arch Otolaryngol Head Neck Surg 2004;130:281-288.

157. Zhu M, Yang T, Wei S, DeWan AT, Morell RJ, Elfenbein JL, Fisher RA, Leal SM, Smith RJH, Friderici KH. Mutations in the gamma-actin gene (ACTG1) are associated with dominant progressive deafness (DFNA20/26). Am J Hum Genet 2003;73:1082-1091.

158. van Wijk E, Krieger E, Kemperman MH, De Leenheer EMR, Huygen PLM, Cremers CWRJ, Cremers FPM, Kremer H. A mutation in the gamma actin 1 (ACTG1) gene causes autosomal dominant hearing loss (DFNA20/26). J Med Genet 2003;40:879-884. 
159. Rendtorff ND, Zhu M, Fagerheim T, Antal TL, Jones M, Teslovich TM, Gillanders EM, Barmada M, Teig E, Trent JM, Friderici KH, Stephan DA, Tranebjaerg L. A novel missense mutation in ACTG1 causes dominant deafness in a Norwegian DFNA20/26 family, but ACTG1 mutations are not frequent among families with hereditary hearing impairment. Eur J Hum Genet 2006;14:1097-1105.

160. Morell RJ, Friderici KH, Wei S, Elfenbein JL, Friedman TB, Fisher RA. A new locus for lateonset, progressive, hereditary hearing loss DFNA20 maps to 17q25. Genomics 2000;63:1-6.

161. Kunst HPM, Marres H, Huygen PLM, Van Duijnhoven G, Krebsova A, Van Der Velde S, Reis A, Cremers F, Cremers CWRJ. Non-syndromic autosomal dominant progressive non-specific mid-frequency sensorineural hearing impairment with childhood to late adolescence onset (DFNA21). Clin. Otolaryng 2000;25:45-54.

162. de Brouwer AP, Kunst HPM, Krebsova A, van Asseldonk K, Reis A, Snoeckx RL, Van Camp G, Cremers CWRJ, Cremers FPM, Kremer H. Fine mapping of autosomal dominant nonsyndromic hearing impairment DFNA21 to chromosome 6p24.1-22.3. Am J Med Genet A 2005;137:41-46.

163. Topsakal V, Hilgert N, Van Dinther J, Vrijens K, Van Camp G, van de Heyning PH. A Belgian family with non-syndromic, autosomal dominant, progressive, sensorineural hearing loss linked to DFNA22. Genetic deafness; A clinical audiological approach. Thesis Topsakal 2006:57-86.

164. Melchionda S, Ahituv N, Bisceglia L, Sobe T, Glaser F, Rabionet R, Arbones ML, Notarangelo A, Di Iorio E, Carella M, Zelante L, Estivill X, Avraham KB, Gasparini P. MYO6, the human homologue of the gene responsible for deafness in Snell's waltzer mice, is mutated in autosomal dominant nonsyndromic hearing loss. Am J Hum Genet 2001;69:635-640.

165. Salam AA, Häfner FM, Linder TE, Spillmann T, Schinzel AA, Leal SM. A novel locus (DFNA23) for prelingual autosomal dominant nonsyndromic hearing loss maps to 14q21-q22 in a Swiss German kindred. Am J Hum Genet 2000;66:1984-1988.

166. Ruf RG, Xu PX, Silvius D, Otto EA, Beekmann F, Muerb UT, Kumar S, Neuhaus TJ, Kemper MJ, Raymond RM Jr, Brophy PD, Berkman J, Gattas M, Hyland V, Ruf EM, Schwartz C, Chang EH, Smith RJ, Stratakis CA, Weil D, Petit C, Hildebrandt F. SIX1 mutations cause branchio-oto-renal syndrome by disruption of EYA1-SIX1-DNA complexes. Proc Natl Acad Sci USA 2004;101:8090-8095.

167. Santos RL, Häfner FM, Huygen PLM, Linder TE, Schinzel AA, Spillmann T, Leal SM. Phenotypic characterization of DFNA24: prelingual progressive sensorineural hearing impairment. Audiol Neurootol 2006;11:269-275.

168. Häfner FM, Salam AA, Linder TE, Balmer D, Baumer A, Schinzel AA, Spillmann T, Leal SM. A novel locus (DFNA24) for prelingual nonprogressive autosomal dominant nonsyndromic hearing loss maps to 4q35-qter in a large Swiss German kindred. Am J Hum Genet 2000;66: 1437-1442.

169. Greene CC, McMillan PM, Barker SE, Kurnool P, Lomax MI, Burmeister M, Lesperance MM. DFNA25, a novel locus for dominant nonsyndromic hereditary hearing impairment, maps to 12q21-24. Am J Hum Genet 2001;68:254-260.

170. Fridell RA, Boger EA, San Agustin T, Brownstein MJ, Friedman TB, Morell RJ: DFNA27, a new locus for autosomal dominant hearing impairment on chromosome 4. Am J Hum Genet 1999;65:A249.

171. Peters LM, Anderson DW, Griffith AJ, Grundfast KM, San Agustin TB, Madeo AC, Friedman TB, Morell RJ. Mutation of a transcription factor, TFCP2L3, causes progressive autosomal dominant hearing loss, DFNA28. Hum Mol Genet 2002;11:2877-2885.

172. Mangino M, Flex E, Capon F, Sangiuolo F, Carraro E, Gualandi F, Mazzoli M, Martini A, Novelli G, Dallapiccola B. Mapping of a new autosomal dominant nonsyndromic hearing loss locus (DFNA30) to chromosome 15q25-26. Eur J Hum Genet 2001;9:667-671. 
173. Ensink RJ, Huygen PLM, Snoeckx RL, Caethoven G, Van Camp G, Cremers CWRJ. A Dutch family with progressive autosomal dominant non-syndromic sensorineural hearing impairment linked to DFNA13. Clin Otolaryngol Allied Sci 2001;26:310-316.

174. Snoeckx RL, Kremer H, Ensink RJ, Flothmann K, de Brouwer A, Smith RJH, Cremers CW, Van Camp G. A novel locus for autosomal dominant non-syndromic hearing loss, DFNA31, maps to chromosome 6p21.3. J Med Genet 2004;41:11-13.

175. Li XC, Saal HM, Friedman TB, Friedman RA: A New Gene For Autosomal Dominant Nonsyndromic Sensorineural Hearing Loss (DFNA32) Maps to 11p15. Am J Hum Genet 2000; 67(suppl 2):314.

176. Kurima K, Szymko Y, Rudy S, Morell RJ, Friedman TB, Griffith AJ: Genetic map localisation of DFNA34 and DFNA36, two autosomal dominant nonsyndromic deafness loci. Am J Hum Genet 2000; 67(suppl 2):300.

177. Makishima T, Kurima K, Brewer CC, Griffith AJ. Early onset and rapid progression of dominant nonsyndromic DFNA36 hearing loss. Otol Neurotol 2004;25:714-719.

178. Kurima K, Peters LM, Yang Y, Riazuddin S, Ahmed ZM, Naz S, Arnaud D, Drury S, Mo J, Makishima T, Ghosh M, Menon PS, Deshmukh D, Oddoux C, Ostrer H, Khan S, Riazuddin S, Deininger PL, Hampton LL, Sullivan SL, Battey JF Jr, Keats BJ, Wilcox ER, Friedman TB, Griffith AJ. Dominant and recessive deafness caused by mutations of a novel gene, TMC1, required for cochlear hair-cell function. Nat Genet 2002;30:277-284.

179. Talebizadeh Z, Kenyon JB, Askew JW, Smith SD : A new locus for dominant progressive hearing loss DFNA37 mapped to chromosome 1p21. Am J Hum Genet 2000; 67(suppl 2):3014.

180. Xiao S, Yu C, Chou X, Yuan W, Wang Y, Bu L, Fu G, Qian M, Yang J, Shi Y, Hu L, Han B, Wang Z, Huang W, Liu J, Chen Z, Zhao G, Kong X. Dentinogenesis imperfecta 1 with or without progressive hearing loss is associated with distinct mutations in DSPP. Nat Genet. 2001;27:201-204. Erratum in: Nat Genet 2001;27:345.

181. Blanton SH, Liang CY, Cai MW, Pandya A, Du LL, Landa B, Mummalanni S, Li KS, Chen ZY, Qin XN, Liu YF, Balkany T, Nance WE, Liu XZ. A novel locus for autosomal dominant nonsyndromic deafness (DFNA41) maps to chromosome 12q24-qter. J Med Genet 2002;39:567570.

182. Xia J, Deng H, Feng Y, Zhang H, Pan Q, Dai H, Long Z, Tang B, Deng H, Chen Y, Zhang R, Zheng D, He Y, Xia K. A novel locus for autosomal dominant nonsyndromic hearing loss identified at 5q31.1-32 in a Chinese pedigree. J Hum Genet 2002;47:635-640.

183. Flex E, Mangino M, Mazzoli M, Martini A, Migliosi V, Colosimo A, Mingarelli R, Pizzuti A, Dallapiccola B. Mapping of a new autosomal dominant non-syndromic hearing loss locus (DFNA43) to chromosome 2p12. J Med Genet 2003;40:278-281.

184. Modamio-Høybjør S, Moreno-Pelayo MA, Mencia A, del Castillo I, Chardenoux S, Armenta D, Lathrop M, Petit C, Moreno F. A novel locus for autosomal dominant nonsyndromic hearing loss (DFNA44) maps to chromosome 3q28-29. Hum Genet 2003;112:242-8.

185. D'Adamo P, Donaudy F, D'Eustacchio A, Di Iorio E, Melchionda S, Gasparini P. A new locus (DFNA47) for autosomal dominant non-syndromic inherited hearing loss maps to 9p21-22 in a large Italian family. Eur J Hum Genet 2003;11:121-124.

186. Donaudy F, Ferrara A, Esposito L, Hertzano R, Ben-David O, Bell RE, Melchionda S, Zelante L, Avraham KB, Gasparini P. Multiple mutations of MYO1A, a cochlear-expressed gene, in sensorineural hearing loss. Am J Hum Genet 2003;72:1571-1577.

187. D'Adamo P, Pinna M, Capobianco S, Cesarani A, D'Eustacchio A, Fogu P, Carella M, Seri M, Gasparini P. A novel autosomal dominant non-syndromic deafness locus (DFNA48) maps to 12q13-q14 in a large Italian family. Hum Genet 2003;112:319-320.

188. Moreno-Pelayo MA, Modamio-Høybjør S, Mencia A, del Castillo I, Chardenoux S, Fernandez-Burriel M, Lathrop M, Petit C, Moreno F. DFNA49, a novel locus for autosomal dominant non-syndromic hearing loss, maps proximal to DFNA7/DFNM1 region on chromosome 1q21-q23. J Med Genet 2003;40:832-836. 
189. Modamio-Høybjør S, Moreno-Pelayo MA, Mencia A, del Castillo I, Chardenoux S, Morais D, Lathrop M, Petit C, Moreno F. A novel locus for autosomal dominant nonsyndromic hearing loss, DFNA50, maps to chromosome 7q32 between the DFNB17 and DFNB13 deafness loci. J Med Genet 2004 Feb;41:e14.

190. Yan D, Ke X, Blanton SH, Ouyang XM, Pandya A, Du LL, Nance WE, Liu XZ. A novel locus for autosomal dominant non-syndromic deafness, DFNA53, maps to chromosome 14q11.2q12. J Med Genet 2006;43:170-174.

191. Gürtler N, Kim Y, Mhatre A, Schlegel C, Mathis A, Lalwani AK. DFNA54, a third locus for low-frequency hearing loss. J Mol Med 2004;82:775-780.

192. Huizing EH, van Bolhuis AH, Odenthal DW. Studies on progressive hereditary perceptive deafness in a family of 335 members. II. Characteristic pattern of hearing deterioration. Acta Otolaryngol 1966;61:161-167.

193. Huizing EH, Odenthal DW, van Bolhuis AH. Results of further studies on progressive hereditary sensorineural hearing loss. Audiology 1972;12:261-263.

194. van den Wijngaart WSIM, Verschuure J, Brocaar MP, Huizing EH. Follow-up study in a family with dominant progressive hereditary sensorineural hearing impairment. I. Analysis of hearing deterioration. Audiology 1985;24:233-240.

195. van den Wijngaart WSIM, Huizing EH, Niermeijer MF, Verschuure J, Brocaar MP, Blom W. Follow-up study in a family with dominant progressive hereditary sensorineural hearing impairment. II. Clinical aspects. Audiology 1985;24:336-342.

196. Thompson DA, Weigel RJ. Characterization of a gene that is inversely correlated with estrogen receptor expression (ICERE-1) in breast carcinomas. Eur J Biochem 1998;252:169-177.

197. Maeda Y, Fukushima K, Kasai N, Maeta M, Nishizaki K. Quantification of TECTA and DFNA5 expression in the developing mouse cochlea. Neuroreport 2001;12:3223-3226.

198. Van Laer L, Vrijens K, Thys S, Van Tendeloo VF, Smith RJH, Van Bockstaele DR, Timmermans JP, Van Camp G. DFNA5: hearing impairment exon instead of hearing impairment gene? J Med Genet 2004;41:401-406.

199. Dunø M, Hove H, Kirchhoff M, Devriendt K, Schwartz M. Mapping genomic deletions down to the base: a quantitative copy number scanning approach used to characterise and clone the breakpoints of a recurrent 7p14.2p15.3 deletion. Hum Genet 2004;115:459-467.

200. Busch-Nentwich E, Söllner C, Roehl H, Nicolson T. The deafness gene dfna5 is crucial for ugdh expression and HA production in the developing ear in zebrafish. Development 2004;131:943951.

201. Haddon CM, Lewis JH. Hyaluronan as a propellant for epithelial movement: the development of semicircular canals in the inner ear of Xenopus. Development 1991;112:541550.

202. Katoh M, Katoh M. Identification and characterization of human DFNA5L, mouse Dfna5l, and rat Dfna5l genes in silico. Int J Oncol 2004;25:765-770.

203. Delmaghani S, del Castillo FJ, Michel V, Leibovici M, Aghaie A, Ron U, Van Laer L, Ben-Tal N, Van Camp G, Weil D, Langa F, Lathrop M, Avan P, Petit C. Mutations in the gene encoding pejvakin, a newly identified protein of the afferent auditory pathway, cause DFNB59 auditory neuropathy. Nat Genet 2006;38:770-778.

204. Grottke C, Mantwill K, Dietel M, Schadendorf D, Lage H. Identification of differentially expressed genes in human melanoma ells with acquired resistance to various antineoplastic drugs. Int J Cancer 2000;88:535-546.

205. Lage H, Helmbach H, Grottke C, Dietel M, Schadendorf D. DFNA5 (ICERE-1) contributes to acquired etoposide resistance in melanoma cells. FEBS Lett 2001;494:54-59.

206. Verhagen WIM, Huygen PLM. Familial progressive vestibulocochlear dysfunction. Arch Neurol 1991;48:262. 
207. Verhagen WIM, Huygen PLM, Bles W. A new autosomal dominant syndrome of idiopathic progressive vestibulo-cochlear dysfunction with middle-age onset. Acta Otolaryngol 1992;112:899-906.

208. Verhagen WIM, Bom SJH, Huygen PLM, Fransen E, Van Camp G, Cremers CWRJ. Familial progressive vestibulocochlear dysfunction caused by a $\mathrm{COCH}$ mutation (DFNA9). Arch Neurol 2000;57:1045-1047.

209. Khetarpal U, Schuknecht HF, Gacek RR, Holmes LB. Autosomal dominant sensorineural hearing loss. Pedigrees, audiologic findings, and temporal bone findings in two kindreds. Arch Otolaryngol Head Neck Surg 1991;117:1032-1042.

210. Halpin C, Khetarpal U, McKenna M. Autosomal-dominant progressive sensorineural hearing loss in a large North American family. Am J Audiol 1996;5:105-111.

211. Fransen E, Verstreken M, Bom SJH, Lemaire F, Kemperman MH, De Kok YJ, Wuyts FL, Verhagen WI, Huygen PL, McGuirt WT, Smith RJ, Van Maldergem LV, Declau F, Cremers $\mathrm{CW}$, Van De Heyning PH, Cremers FP, Van Camp G. A common ancestor for $\mathrm{COCH}$ related cochleovestibular (DFNA9) patients in Belgium and The Netherlands bearing the P51S mutation. J Med Genet 2001;38:61-65.

212. Merchant SN, Linthicum FH, Nadol JB Jr. Histopathology of the inner ear in DFNA9. In: Kitamura K, Steel KP (eds). Genetics in Otorhinolaryngology. Advances in Oto-RhinoLaryngology, vol. 56. Basel: Karger 2000;56:212-217.

213. Eavey RD, Manolis EN, Lubianca J, Merchant S, Seidman JG, Seidman C. Mutations in COCH (formerly Coch5b2) cause DFNA9. In: Kitamura K, Steel KP (eds). Genetics in Otorhinolaryngology. Advances in Oto-Rhino-Laryngology, vol. 56. Basel: Karger 2000;56: 101-102.

214. Vermeire K, Brokx JP, Wuyts FL, Cochet E, Hofkens A, De Bodt M, Van de Heyning PH. Good speech recognition and quality-of-life scores after cochlear implantation in patients with DFNA9. Otol Neurotol 2006;27:44-49.

215. Khetarpal U. DFNA9 is a progressive audiovestibular dysfunction with a microfibrillar deposit in the inner ear. Laryngoscope 2000;110:1379-1384.

216. Sanchez E, Lopez-Escamez JA, Lopez-Nevot MA, Lopez-Nevot A, Cortes R, Martin J. Absence of $\mathrm{COCH}$ mutations in patients with Meniere disease. Eur J Hum Genet 2004;12:7578.

217. Bhattacharya SK, Rockwood EJ, Smith SD, Bonilha VL, Crabb JS, Kuchtey RW, Robertson NG, Peachey NS, Morton CC, Crabb JW. Proteomics reveal cochlin deposits associated with glaucomatous trabecular meshwork. J Biol Chem 2005;280:6080-6084.

218. Bhattacharya SK, Annangudi SP, Salomon RG, Kuchtey RW, Peachey NS, Crabb JW. Cochlin deposits in the trabecular meshwork of the glaucomatous DBA/2J mouse. Exp Eye Res 2005;80:741-744.

219. Vihtelic TS, Fadool JM, Gao J, Thornton KA, Hyde DR, Wistow G. Expressed sequence tag analysis of zebrafish eye tissues for NEIBank. Mol Vis 2005;11:1083-1100.

220. Robertson NG, Khetarpal U, Gutierrez-Espeleta GA, Bieber FR, Morton CC. Isolation of novel and known genes from a human fetal cochlear cDNA library using subtractive hybridization and differential screening. Genomics. 1994;23:42-50.

221. Robertson NG, Skvorak AB, Yin Y, Weremowicz S, Johnson KR, Kovatch KA, Battey JF, Bieber FR, Morton CC. Mapping and characterization of a novel cochlear gene in human and in mouse: a positional candidate gene for a deafness disorder, DFNA9. Genomics 1997;46:345354.

222. Robertson NG, Resendes BL, Lin JS, Lee C, Aster JC, Adams JC, Morton CC. Inner ear localisation of mRNA and protein products of $\mathrm{COCH}$, mutated in the sensorineural deafness and vestibular disorder, DFNA9. Hum Mol Genet 2001;10:2493-2500. 
223. Colombatti A, Bonaldo P. The superfamily of proteins with von Willebrand factor type A-like domains: one theme common to components of extracellular matrix, hemostasis, cellular adhesion, and defense mechanisms. Blood 1991;77:2305-2315.

224. Trexler M, Banyai L, Patthy L. The LCCL module. Eur J Biochem 2000;267:5751-5757.

225. Nakamura T, Tokunaga F, Morita T, Iwanaga S, Kusumoto S, Shiba T, Kobayashi T, Inoue K. Intracellular serine-protease zymogen, factor $\mathrm{C}$, from horseshoe crab hemocytes. Its activation by synthetic lipid A analogues and acidic phospholipids. Eur J Biochem 1988;176:89-94.

226. Liepinsh E, Trexler M, Kaikkonen A, Weigelt J, Banyai L, Patthy L, Otting G. NMR structure of the LCCL domain and implications for DFNA9 deafness disorder. EMBO J 2001;20:53475353.

227. Robertson NG, Cremers CWRJ, Huygen PLM, Ikezono T, Krastins B, Kremer H, Kuo SF, Liberman MC, Merchant SN, Miller CE, Nadol JB Jr, Sarracino DA, Verhagen WI, Morton CC. Cochlin immunostaining of inner ear pathologic deposits and proteomic analysis in DFNA9 deafness and vestibular dysfunction. Hum Mol Genet 2006;15:1071-1085.

228. Robertson NG, Hamaker SA, Patriub V, Aster JC, Morton CC. Subcellular localisation, secretion, and post-translational processing of normal cochlin, and of mutants causing the sensorineural deafness and vestibular disorder, DFNA9. J Med Genet 2003;40:479-486.

229. Ikezono T, Omori A, Ichinose S, Pawankar R, Watanabe A, Yagi T. Identification of the protein product of the Coch gene (hereditary deafness gene) as the major component of bovine inner ear protein. Biochim Biophys Acta 2001;1535:258-265.

230. Makishima T, Rodriguez CI, Robertson NG, Morton CC, Stewart CL, Griffith AJ. Targeted disruption of mouse Coch provides functional evidence that DFNA9 hearing loss is not a COCH haploinsufficiency disorder. Hum Genet 2005;118:29-34.

231. Khetarpal U. Autosomal dominant sensorineural hearing loss: further temporal bone findings. Arch Otolaryngol Head Neck Surg 1993;119:106-108.

232. Boulassel MR, Tomasi JP, Deggouj N, Gersdorff M. COCH5B2 is a target antigen of anti-inner ear antibodies in autoimmune inner ear diseases. Otol Neurotol 2001;22:614-618.

233. Billings P. Experimental autoimmune hearing loss. J Clin Invest 2004;113:1114-1117.

234. Solares CA, Edling AE, Johnson JM, Baek MJ, Hirose K, Hughes GB, Tuohy VK. Murine autoimmune hearing loss mediated by CD4+ $\mathrm{T}$ cells specific for inner ear peptides. J Clin Invest 2004;113:1210-1217.

235. Baek MJ, Park HM, Johnson JM, Altuntas CZ, Jane-Wit D, Jaini R, Solares CA, Thomas DM, Ball EJ, Robertson NG, Morton CC, Hughes GB, Tuohy VK. Increased frequencies of cochlinspecific $\mathrm{T}$ cells in patients with autoimmune sensorineural hearing loss. J Immunol 2006;177:4203-4210.

236. Ishiyama A, Ishiyama G, Lopez I, Jen J, Kim G, Baloh RW. Temporal bone histopathology in dominantly inherited audiovestibular syndrome. Neurology 2004;63:1859-1862.

237. Udovichenko IP, Gibbs D, Williams DS. Actin-based motor properties of native myosin VIIa. J Cell Sci 2002;115:445-450.

238. Chen ZY, Hasson T, Kelley PM, Schwender BJ, Schwartz MF, Ramakrishnan M, Kimberling WJ, Mooseker MS, Corey DP. Molecular cloning and domain structure of human myosinVIIa, the gene product defective in Usher syndrome 1B. Genomics 1996;36:440-448.

239. Weil D, Levy G, Sahly I, Levi-Acobas F, Blanchard S, El-Amraoui A, Crozet F, Philippe H, Abitbol M, Petit C. Human myosin VIIA responsible for the Usher 1B syndrome: a predicted membrane-associated motor protein expressed in developing sensory epithelia. Proc Natl Acad Sci USA 1996;93:3232-3237.

240. Chishti AH, Kim AC, Marfatia SM, Lutchman M, Hanspal M, Jindal H, Liu SC, Low PS, Rouleau GA, Mohandas N, Chasis JA, Conboy JG, Gascard P, Takakuwa Y, Huang SC, Benz EJ Jr, Bretscher A, Fehon RG, Gusella JF, Ramesh V, Solomon F, Marchesi VT, Tsukita S, Tsukita S, Arpin M, Louvard D, Tonks NK, Anderson JM, Fanning AS, Bryant PJ, Woods DF, 
Hoover KB. The FERM domain: a unique module involved in the linkage of cytoplasmic proteins to the membrane. Trends Biochem Sci 1998;23:281-282.

241. Etournay R, El-Amraoui A, Bahloul A, Blanchard S, Roux I, Pezeron G, Michalski N, Daviet L, Hardelin JP, Legrain P, Petit C. PHR1, an integral membrane protein of the inner ear sensory cells, directly interacts with myosin 1c and myosin VIIa. J Cell Sci 2005;118:2891-2899.

242. Hasson T, Gillespie PG, Garcia JA, MacDonald RB, Zhao Y, Yee AG, Mooseker MS, Corey DP. Unconvential myosins in inner-ear sensory epithelia. J Cell Biol 1997;137:1287-1307.

243. Sahly I, El-Amraoui A, Abitbol M, Petit C, Dufier JL. Expression of myosin VIIA during mouse embryogenesis. Anat Embryol 1997;196:159-170.

244. Wolfrum U, Liu X, Schmitt A, Udovichenko IP, Williams DS. Myosin VIIa as a common component of cilia and microvilli. Cell Motil Cytoskel 1998;40:261-271.

245. Hasson T, Heintzelman MB, Santos-Sacchie J, Corey DP, Mooseker MS. Expression in cochlea and retina of myosin VIIa, the gene product defective in Usher syndrome type 1B. Proc Natl Acad Sci USA 1995;92:9815-9819.

246. El-Amraoui A, Sahly I, Picaud S, Sahel J, Abitbol M, Petit C. Human Usher 1B/mouse shaker1: the retinal phenotype discrepancy explained by the presence/absence of myosin VIIA in the photoreceptor cells. Hum Mol Genet 1996;5:1171-1178.

247. Liu X, Vansant G, Udovichenko IP, Wolfrum U, Williams DS. Myosin VIIa, the product of the Usher 1B syndrome gene, is concentrated in the connecting cilia of photoreceptor cells. Cell Motil Cytoskeleton 1997;37:240-252.

248. Wolfrum U. The cellular function of the usher gene product myosin VIIa is specified by its ligands. Adv Exp Med Biol 2003;533:133-142.

249. Reiners J, Nagel-Wolfrum K, Jurgens K, Marker T, Wolfrum U. Molecular basis of human Usher syndrome: deciphering the meshes of the Usher protein network provides insights into the pathomechanisms of the Usher disease. Exp Eye Res 2006;83:97-119.

250. Self T, Mahony M, Fleming J, Walsh J, Brown SDM, Steel KP. Shaker-1 mutations reveal roles for myosin VIIA in both development and function of cochlear hair cells. Development 1998;125:557-566.

251. Boëda B, El-Amraoui A, Bahloul A, Goodyear R, Daviet L, Blanchard S, Perfettini I, Fath KR, Shorte S, Reiners J, Houdusse A, Legrain P, Wolfrum U, Richardson G, Petit C. Myosin VIIa, harmonin and cadherin 23, three Usher I gene products that cooperate to shape the sensory hair cell bundle. EMBO J 2002;21:6689-6699.

252. Adato A, Michel V, Kikkawa Y, Reiners J, Alagramam KN, Weil D, Yonekawa H, Wolfrum U, El-Amraoui A, Petit C. Interactions in the network of Usher syndrome type 1 proteins. Hum Mol Genet 2005;14:347-356.

253. Kros CJ, Marcotti W, van Netten SM, Self TJ, Libby RT, Brown SD, Richardson GP, Steel KP. Reduced climbing and increased slipping adaptation in cochlear hair cells of mice with Myo7a mutations. Nat Neurosci 2002;5:41-47.

254. Siemens J, Lillo C, Dumont RA, Reynolds A, Williams DS, Gillespie PG, Muller U. Cadherin 23 is a component of the tip link in hair-cell stereocilia. Nature 2004;428:950-955.

255. Reiners J, van Wijk E, Marker T, Zimmermann U, Jurgens K, te Brinke H, Overlack N, Roepman R, Knipper M, Kremer H, Wolfrum U. Scaffold protein harmonin (USH1C) provides molecular links between Usher syndrome type 1 and type 2. Hum Mol Genet 2005;14:3933-3943.

256. Liu X, Ondek B, Williams DS. Mutant myosin VIIa causes defective melanosome distribution in the RPE of shaker-1 mice. Nat Genet 1998;19:117-118.

257. El-Amraoui A, Schonn JS, Kussel-Andermann P, Blanchard S, Desnos C, Henry JP, Wolfrum U, Darchen F, Petit C. MyRIP, a novel Rab effector, enables myosin VIIa recruitment to retinal melanosomes. EMBO Rep 2002;3:463-470. 
258. Gibbs D, Azarian SM, Lillo C, Kitamoto J, Klomp AE, Steel KP, Libby RT, Williams DS. Role of myosin VIIa and Rab27a in the motility and localisation of RPE melanosomes. J Cell Sci 2004;117:6473-6483.

259. Gibbs D, Kitamoto J, Williams DS. Abnormal phagocytosis by retinal pigmented epithelium that lacks myosin VIIa, the Usher syndrome 1B protein. Proc Natl Acad Sci USA 2003;100:6481-6486.

260. Liu X, Udovichenko IP, Brown SDM, Steel KP, Williams DS. Myosin VIIa participates in opsin transport through the photoreceptor cilium. J Neurosci 1999;19:6267-6274.

261. Kussel-Andermann P, El-Amraoui A, Safieddine S, Nouaille S, Perfettini I, Lecuit M, Cossart $\mathrm{P}$, Wolfrum U, Petit C. Vezatin, a novel transmembrane protein, bridges myosin VIIA to the cadherin-catenins complex. EMBO J 2000;19:6020-6029.

262. Gibson F, Walsh J, Mburu P, Varela A, Brown KA, Antonio M, Beisel KW, Steel KP, Brown SD. A type VII myosin encoded by the mouse deafness gene shaker-1. Nature 1995;374:62-64.

263. Rhodes CR, Hertzano R, Fuchs H, Bell RE, de Angelis MH, Steel KP, Avraham KB. A Myo7a mutation cosegregates with stereocilia defects and low-frequency hearing impairment. Mamm Genome 2004;15:686-697. 



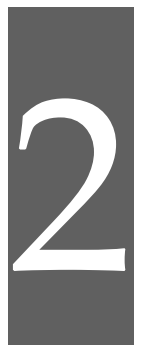

DFNA5 



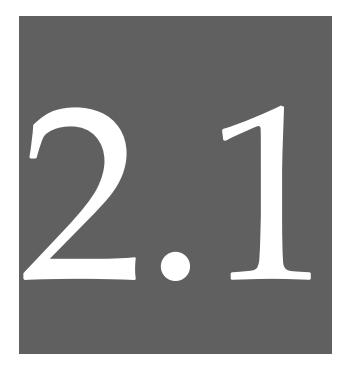

\title{
A novel mutation identified in the DFNA5 gene in a Dutch family: A clinical and genetic evaluation.
}

\author{
*A.M.L.C. Bischoff \\ *M.W.J. Luijendijk \\ P.L.M. Huygen \\ G. van Duijnhoven \\ E.M.R. De Leenheer \\ G.G. Oudesluijs \\ L. Van Laer \\ F.P.M. Cremers \\ C.W.R.J. Cremers \\ H. Kremer
}





\begin{abstract}
A novel DFNA5 mutation was found in a Dutch family, of which 37 members were examined. A nucleotide substitution was identified in the splice-acceptor site of intron 7, leading to skipping of exon 8 in part of the transcripts. The mutation was found in 18 individuals. Sensorineural hearing impairment was nonsyndromic and symmetric. In early life, presumably congenital, hearing impairment amounted to $30 \mathrm{~dB}$ in the high frequencies. Progression was most pronounced at $1 \mathrm{kHz}(1.8 \mathrm{~dB} / \mathrm{y})$. Speech recognition was relatively good with a phoneme score of about $50 \%$ at age 70 . Onset age was 37 years and recognition deteriorated by $1.3 \%$ per year. The recognition score deteriorated by $1.0 \%$ per $\mathrm{dB}$ threshold increase from a mean pure tone average (PTA 1,2,4 kHz) of $63 \mathrm{~dB}$ onwards. Vestibular function was generally normal. The second mutation identified in the DFNA5 gene results in hearing impairment, similar to that in the original DFNA5 family in terms of pure tone thresholds, but with more favourable speech recognition.
\end{abstract}

\title{
Introduction
}

Since the early nineties, gene linkage and mutation analysis have been applied to increase our knowledge of hereditary non-syndromic hearing impairment. At the moment about 75 genetically different types of non-syndromic hearing impairment have been identified. ${ }^{1}$ Knowledge of hereditary hearing impairment at a molecular level is still limited, but can be enhanced by studies on genotypephenotype correlations in families with hearing impairment. ${ }^{2}$ This is especially true for autosomal dominant types, which display the largest phenotypic variation. The results of such family studies are also of value for genetic counselling purposes.

Up to now, only one DFNA5 family has been described..$^{3-9}$ In this family, nonsyndromic symmetric hearing impairment was most severe at the high frequencies and progression was most distinct in the first three decades of life. In 1995 the responsible gene was localized to a region on chromosome 7p15. The locus was designated DFNA5. ${ }^{10}$ After sequencing of the DFNA5 gene, a complex insertion/deletion mutation was found in intron 7, causing skipping of exon 8 and leading to premature termination of the encoded protein. As there was no indication for its function, the gene was called DFNA5 by default. ${ }^{11}$ The DFNA5 protein is not homologous to any other human protein described up to now. Also no information is available about the structure or the subcellular localization of the protein. 
In this report we describe a second mutation in the DFNA5 gene in a Dutch family with autosomal dominant sensorineural hearing impairment. A nucleotide substitution was identified in the splice-acceptor site of intron 7, which leads to alternative splicing. This results in skipping of exon 8 in part of the transcripts. Pure tone and speech audiometric data were analysed and compared to the results in the previously described DFNA5 family. ${ }^{12}$ Although a larger part of the transcripts is normal in comparison to the original DFNA5 family, the severity of hearing impairment is fairly similar in terms of pure tone hearing thresholds.

\section{Patients and methods}

A five-generation pedigree was established for the present family (W00-212). Thirty-seven family members participated in this study. The medical history was taken and otologic examination was performed. Attention was paid to the presence of any syndromic features. Non-hereditary causes of hearing loss were excluded and written informed consent was obtained. All individuals included in this study underwent pure tone audiometry; speech audiometry was performed in affected persons only. Vestibular function was tested in six cases. One affected person underwent computerized tomography of the temporal bones. Blood samples were obtained for linkage analysis from 18 presumably affected family members and 19 presumably unaffected persons.

\section{Audiometric analysis}

Pure tone and speech audiometry were performed in a sound treated room, conforming to the International Standards Organization (ISO) standards. ${ }^{13,14}$ The individual $95^{\text {th }}$ percentile threshold values of presbyacusis $\left(\mathrm{P}_{95}\right)$ in relation to the patient's sex and age were derived for each frequency using the ISO 7029 method. ${ }^{15}$ Persons were considered affected if the best hearing ear showed thresholds beyond the $P_{95}$. Mean binaural threshold levels were used to perform longitudinal regression analyses. Analysis of covariance (ANCOVA, Prism PC version 3.02; GraphPad, San Diego CA, USA) was performed to compare between slopes and intercepts of the regression lines pertaining to individual measurements per frequency. Where pooled regression was inappropriate, the median slope (called annual threshold deterioration, ATD, expressed in $\mathrm{dB} /$ year) and intercept were calculated, following inspection of the corresponding frequency distributions. Outlying values were excluded, if necessary. The lines defined by the combination of the slope and intercept values thus obtained were called trend lines. These lines were used to construct Age Related Typical 
Audiograms (ARTA). To compare the audiological data of the original ${ }^{12}$ and the present DFNA5 family, we transformed the ARTA into a one-dimensional threshold features array, which can be used for chi-square analysis, as previously described. ${ }^{2}$

\section{Speech audiometry}

Speech audiometry was performed, using standard monosyllabic Dutch word lists. The maximum monaural phoneme score (\% correct recognition) was derived from a performance versus intensity plot. Cross-sectional binaural mean phoneme recognition scores were plotted for all mutation carriers against age and binaural mean PTA $1,2,4 \mathrm{kHz}$ (pure tone average in $\mathrm{dB}$ hearing level). Non-linear regression analysis was used to fit sigmoidal dose-response curves with variable slope, as previously described. ${ }^{16}$ Outlying values were excluded, if necessary. The age of onset and onset level were defined at a maximum phoneme recognition score of $90 \%$ in the performance versus age and performance versus impairment plots. The slope was called deterioration rate in the performance versus age plot, whereas it was called deterioration gradient in the performance versus impairment plot. To simplify the results and allow for additional testing, "local average" slope (i.e. on a linear scale) for $X>X_{90}(X$ is either age or PTA level) was obtained by fitting a straight line as previously described, ${ }^{17}$ using a non-linear method to estimate $X_{90 .}{ }^{18}$ Student's $t$ test (with Welch correction if significantly unequal variance was detected by Bartlett's test) was employed to test age of onset, deterioration rate, onset level or deterioration gradient between the present and the original DFNA5 family. ${ }^{18}$

\section{Linkage analysis}

DNA was isolated from peripheral blood according to Miller et al. ${ }^{19}$ Analysis of the micro satellite markers was performed as described by Kremer et al. ${ }^{20}$ Twopoint lod scores were calculated with the MLINK subroutine of the LINKAGE package version 5.1. ${ }^{21-23}$ A penetrance of 0.95, a disease allele frequency of 0.0001 and a phenocopy rate of 0.001 were assumed for the calculations.

\section{Mutation analysis}

Amplification of the protein-coding region from chromosomal DNA was performed using standard PCR conditions. Primer sequences have been described by van Laer et al., ${ }^{24}$ except for amplification of exon 9 for which we used 5'GGTCCCACCTGGGAGGTTGC-3' and 5'-CTGTGACTGTGATTTTTCTCTCC-3'. Prior to sequencing, PCR fragments were purified with the QIAquick gel 
extraction kit (Qiagen). Sequence analysis was performed using the ABI PRISM Big Dye Terminator cycle sequencing V2.0 ready reaction kit and an ABI PRISM 3700 DNA analyser (Applied Bio systems). For testing the segregation of the mutation in the family genomic DNA was amplified with the primers $5^{\prime}$ GTCTCCAGCTGTGTCATGACC-3' and 5'-CCATTTCTTTCATTTTCTTTTCTCC3'. PCR fragments were digested with RsaI (Invitrogen) and separated on an agarose gel (Seakem agarose, FMC).

\section{Reverse transcriptase polymerase chain reaction}

Messenger RNA of lymphoblastoid cells cultured under standard conditions was isolated according to the RNA-Bee (Campro Scientific) protocol.

To establish the effect of nonsense mediated RNA decay (NMD), lymphoblastoid cells were treated with cycloheximide $(100 \mu \mathrm{g} / \mathrm{mL}$ cell culture medium) for 4.5 hours. For the initial testing of skipping of exon 8 and detection of other alternative splicing products, cDNA was synthesized by random priming with MMLV reverse transcriptase (Invitrogen). Subsequently PCR was performed with the primers 5'-CCTGAGCCACAACAGACAGC-3' in exon 7 and 5' GGCACAGTGTGGGAATGATCTG-3' in exon 9 and fragments were analysed on agarose gel. For the detection of alternative splicing in other parts of the mRNA, the following primers were used: 5' -AGAGGCCCCGACATCTCC-3' in exon 1, 5' TCGACCTGCATGTGCTCAG-3' in exon 4, 5' -GGTGCTGGAAGGAAGGAATG$3^{\prime}$ in exon 4, 5'-CAAATAGGACCGCCTGGAAG-3' in exon 7, 5'TCTGCTGGGCACTTGCTG-3' in exon 9 and ${ }^{\prime}$ TCATCATGCAAAATGTCACCAC-3' in exon 10. For comparison of the ratio between the normal and the short DFNA5 mRNA, cDNA synthesis was performed with the One-Step RT-PCR kit (Qiagen). The ${ }^{32} \mathrm{P}$-labelled primers given above for testing of exon 8 skipping were used and 25 cycles of PCR were performed. After denaturation, fragments were separated on a denaturing acrylamide gel. Phospo-imaging was performed using the Gs-363 molecular imaging system (Biorad). The data were analysed and the ratios between normal and short mRNA were determined with the molecular analyst software, version 1.5 (Biorad). As control samples for RT-PCR, mRNA was isolated from lymphoblastoid cells of 22 normal-hearing individuals. One individual was 15 years old, all others were between 20 and 83 years old.

\section{Southern blot analysis}

Chromosomal DNA (about $8 \mu \mathrm{g}$ per lane) was digested with MspI, EcoRI and TaqI and separated on a $0.8 \%$ agarose gel (Seakem agarose, FMC) and blotted onto 
GeneScreen plus (NEN Life Science Products) by a standard dry blot transfer method. As probe, $40 \mathrm{ng}$ of a cDNA encompassing the complete coding region was labelled with $\alpha\left[{ }^{32} \mathrm{P}\right] \mathrm{dCTP}$ by random primer extension and hybridized under standard conditions.

\section{Results}

The pedigree (Figure 1) comprised 27 affected family members, 18 of whom were still alive and participated in this study. The pattern of inheritance is clearly autosomal dominant.

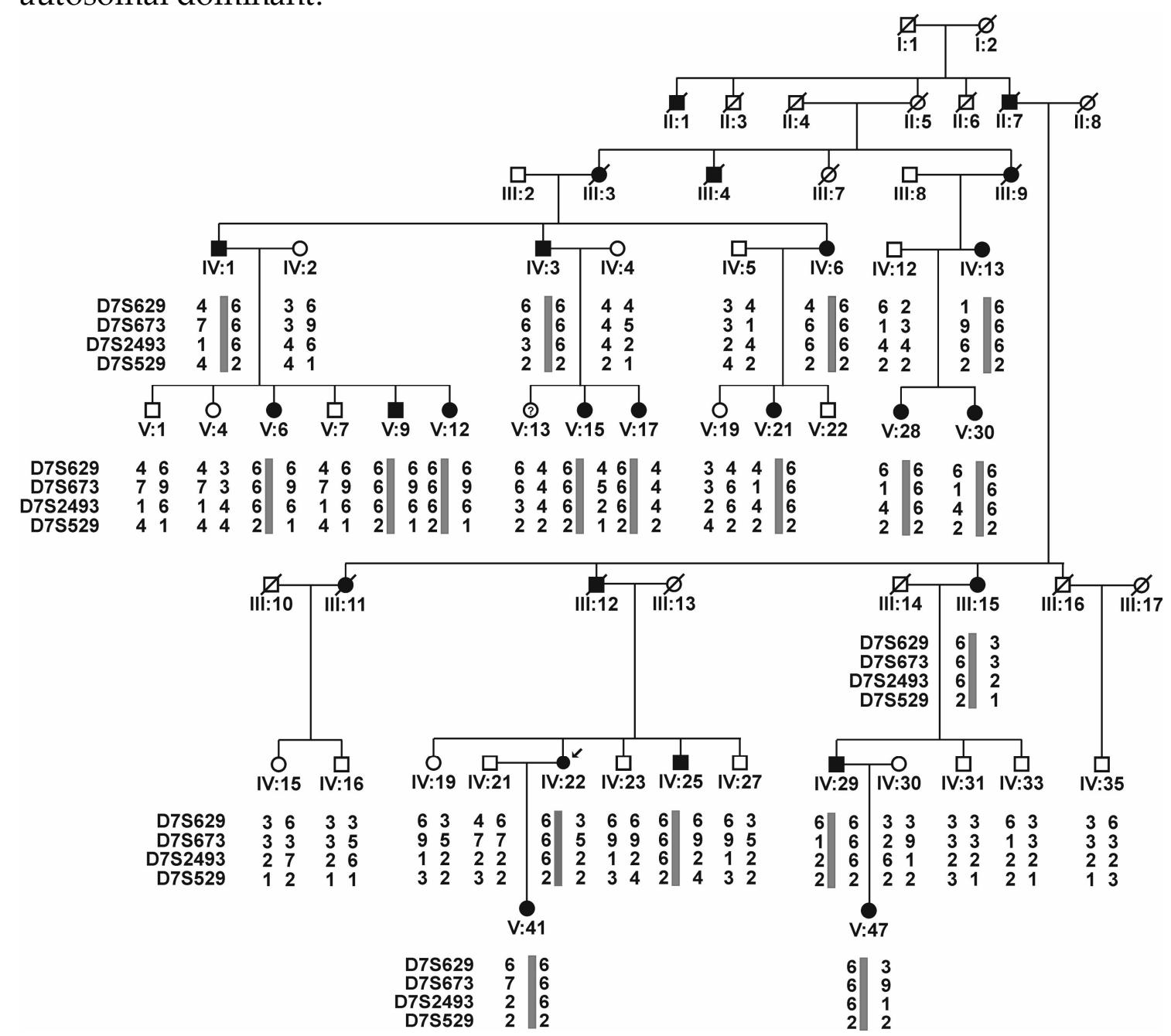

Figure 1. Pedigree of family W00-212 and genotypic data for markers flanking the DFNA5 gene, listed in centromere-to-telomere order. The most likely haplotypes are shown. A bar indicates the haplotype that is associated with the affected status. The marker order is in agreement with the most recent freeze (June 2002) of the Human Genome Working Draft (http://genome.ucse.edu). Men are indicated with squares, women are indicated with circles and filled symbols indicate affected persons. The proband is indicated with an arrow. Haplotype reconstruction for the individuals III:2 and III:3 suggests the presence of five alleles. However, assuming one allele change for marker D7S2493 for either individual IV:3 or IV:6 can explain this. 

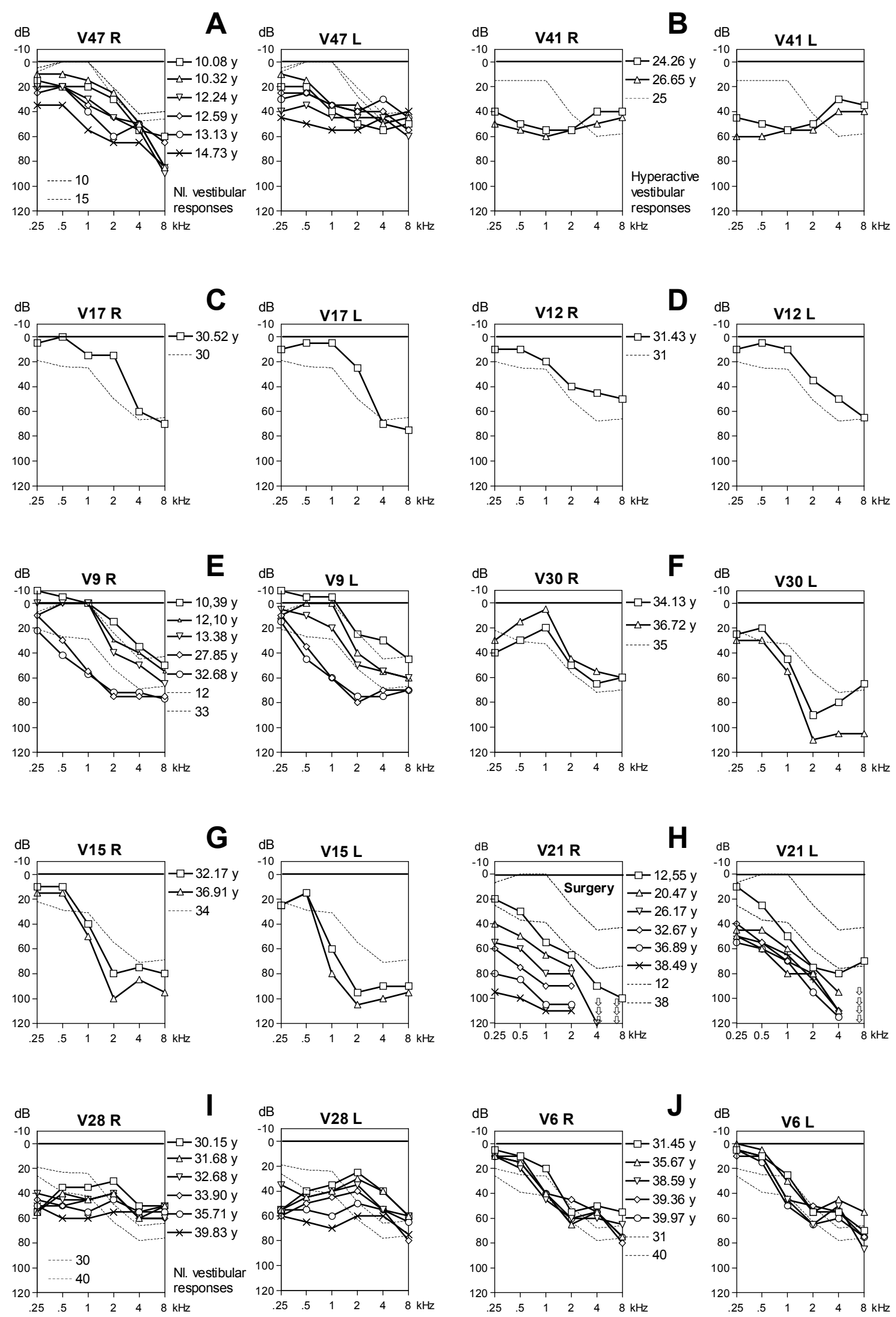

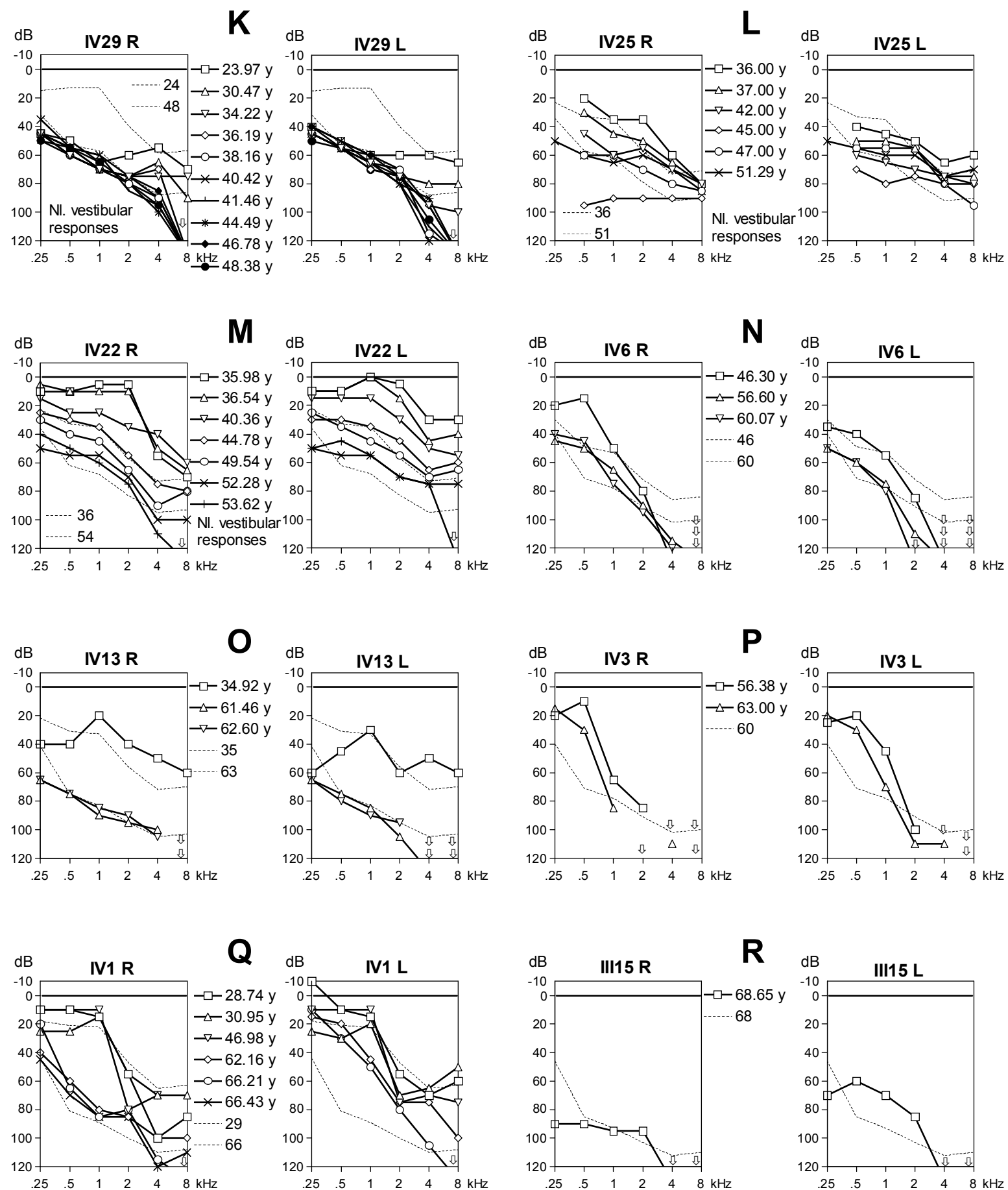

Figure 2. Audiograms of the 18 affected family members. $\mathrm{Nl}=$ normal. The dotted lines represent the predicted thresholds at the ages specified, as derived from the ARTA. The results of the six vestibulo-ocular function tests are also indicated. 
Affected persons showed no evidence of other causes of hearing impairment than otitis in the past, except for case V:21, who had a cholesteatoma in the right ear at 14 years of age that required surgery. The data for the right ear in this individual were therefore excluded from the present analysis. The first symptoms of hearing impairment were reported at ages ranging from 0 to 40 years. Vestibular symptoms were not reported. Otoscopy was generally normal. Pure tone audiograms (Figure 2) showed a so-called Z-shape curve; predominantly high frequency hearing loss with a large drop for the mid-frequencies and relatively sparing of the lower frequencies. The audiograms were fairly symmetric, except for cases V:21 and V:30.

\section{Audiometric analysis}

All available data, combining individual longitudinal (11 cases) and single snapshot (7 cases) measurements are shown in Figure 3. As ANCOVA indicated that pooling of regression lines was not allowed for any frequency, the trend lines were derived. At each frequency, it was checked that fairly similar numbers of individuals were represented by threshold values - either pertaining to longitudinal or snapshot observations - above and below the trend line. The ATD increased from $0.7 \mathrm{~dB} /$ year at $0.25 \mathrm{kHz}$ to $1.8 \mathrm{~dB} /$ year at $1 \mathrm{kHz}$ and then decreased to $1.2 \mathrm{~dB} /$ year at $4-8 \mathrm{kHz}$.

The constructed ARTA (Figure 4) show a configuration with congenital high frequency hearing impairment of about $30 \mathrm{~dB}$ and progression that was most pronounced at 0.5 and $1 \mathrm{kHz}$. To check whether the constructed ARTA was reliable, we plotted the thresholds predicted for minimum and maximum age, derived from the ARTA, in the original audiograms (Figure 2, dotted lines). This highlighted threshold variability, especially in the younger individuals, who seemed to be more severely affected than was indicated by their expected audiograms. Attempts to improve prediction for the younger patients only succeeded at the cost of the quality of prediction in the remaining cases and were therefore abandoned.

In Figure 4, the ARTA published for the original DFNA5 family ${ }^{12}$ is illustrated, in juxtaposition with the ARTA derived for the present family. For both families, we created a threshold features array as described elsewhere. ${ }^{2}$ A chi-square test did not detect a significant difference between the families, although in the first four decades the ARTA of the present family exhibited better thresholds at the speech frequencies. 

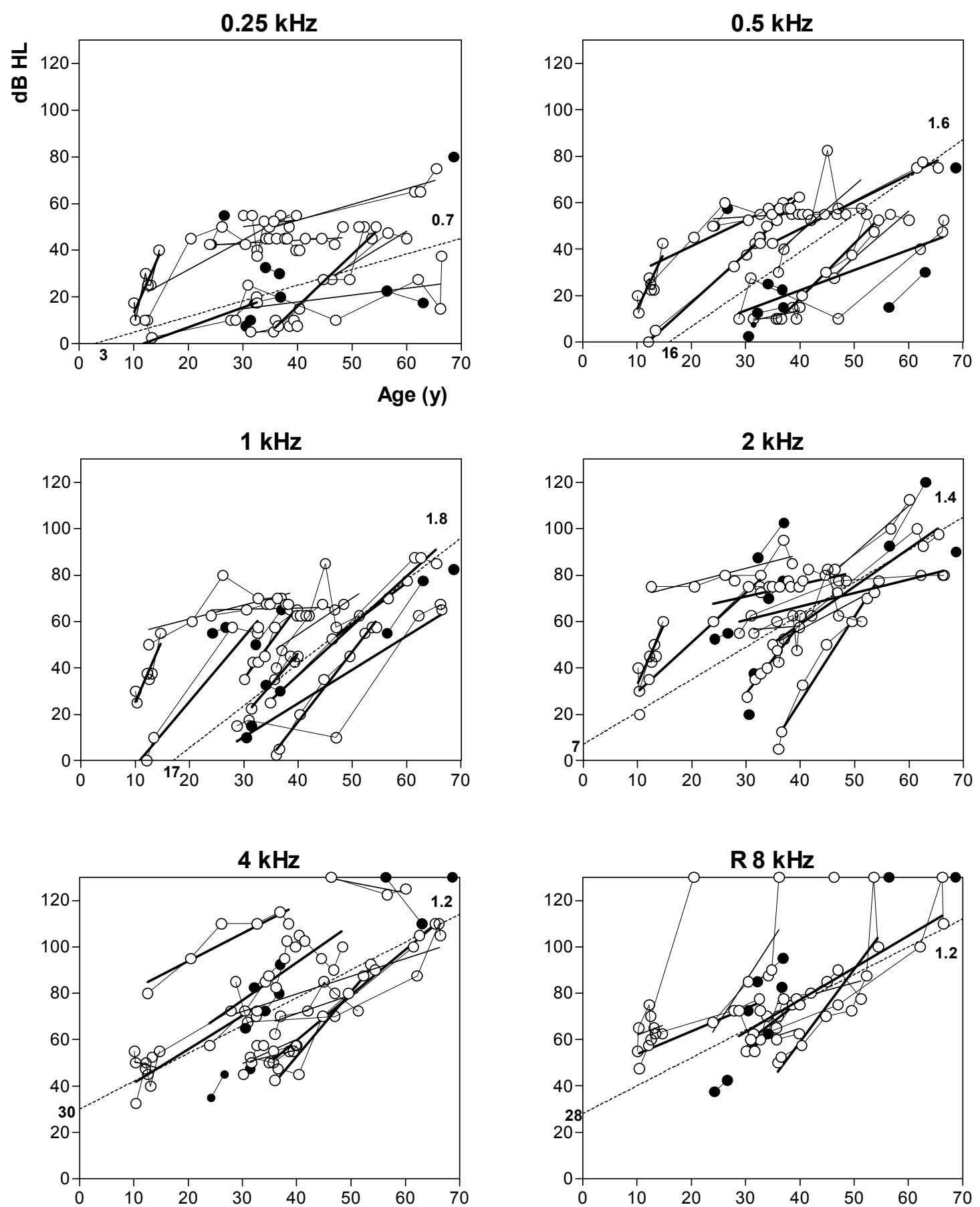

Figure 3. Longitudinal analysis (binaural air conduction threshold). Threshold data (in $\mathrm{dB}$ hearing level) versus age (in years) for the frequencies from 0.25 to $8 \mathrm{kHz}$ combining individual longitudinal data (open circles) with connecting hairlines and single snapshot measurements (filled circles) of the 18 mutation carriers. The regression lines fitted to the individual longitudinal measurements are included (a bold line indicates significant progression). The dotted line pertains to the trend line derived for the ensemble of these data points (see text). Slopes and intercepts are indicated in bold. 
We checked for the presence of phenotypic differences related to gender since the expression of DFNA5, also known as ICERE-1, is higher in estrogen-receptornegative than in estrogen-receptor-positive breast carcinomas; ${ }^{25}$ none were found.

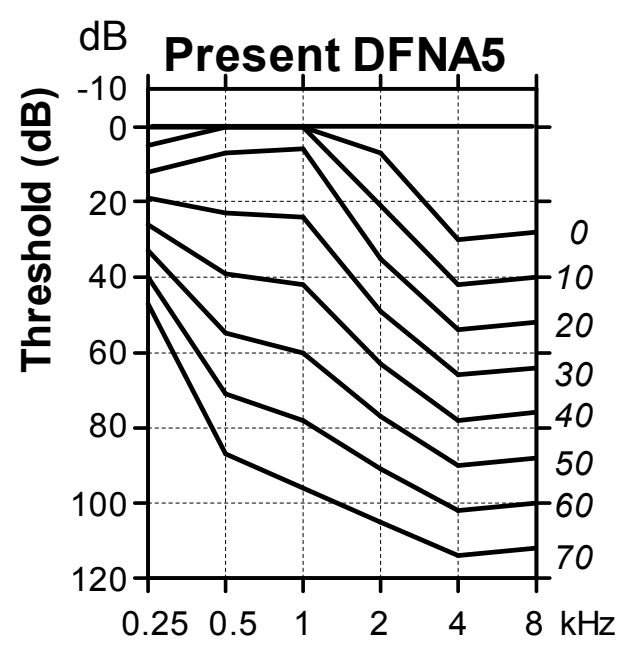

Frequency $(\mathbf{k H z})$

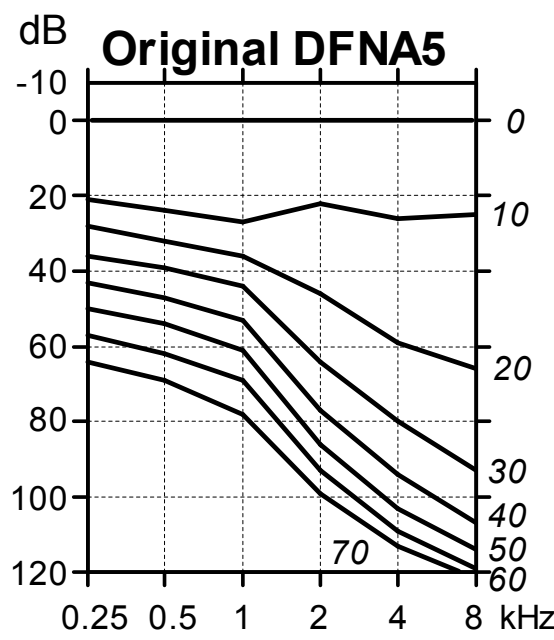

Figure 4. ARTA of the original and the present DFNA5 family from birth to the 7th decade. Italics indicate age (years) in decade steps.

A

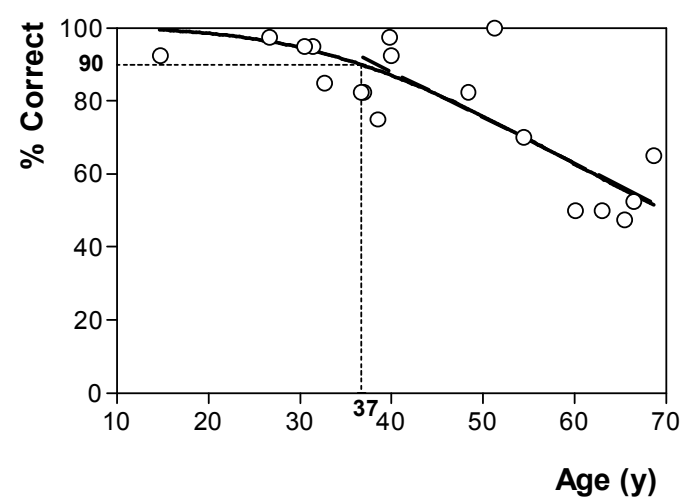

B

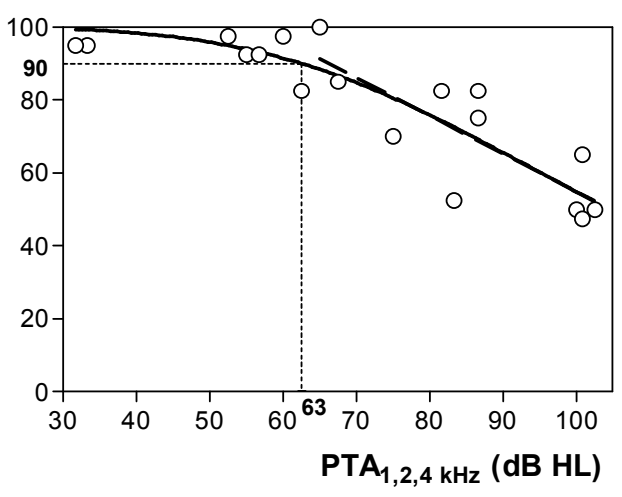

Figure 5. Cross-sectional binaural mean speech recognition scores at last visit against age (A) and PTA at 1, 2 and $4 \mathrm{kHz}$ (B). The continuous curve was fitted using a non-linear equation. A straight line (dashed) approximated the relevant part of this curve, see methods. The onset age and onset level were derived, using the non-linear curve.

\section{Speech recognition}

Speech recognition was relatively good, with scores still better than $50 \%$ at the age of 70 . The recognition score (Figure 5A) declined substantially from onset at the age of 37 with a deterioration rate of $1.3 \%$ per year (Table 1 ). The $90 \%$ recognition score was found at a PTA level of $63 \mathrm{~dB}$ hearing level and deteriorated by about $1.0 \%$ per $\mathrm{dB}$ (Figure $5 \mathrm{~B}$ ). 
Table 1. Speech recognition scores

\begin{tabular}{|c|c|c|c|c|}
\hline & $\begin{array}{l}\text { Present } \\
\text { DFNA5 }\end{array}$ & $\begin{array}{l}\text { Original } \\
\text { DFNA5 }\end{array}$ & DFNA2 & DFNA9 \\
\hline \multicolumn{5}{|l|}{ Age, years } \\
\hline $\mathrm{n}$ & 12 & 33 & 43 & 41 \\
\hline Onset age & $38(28-49) \quad S$ & $16(10-22)$ & 34 & 43 \\
\hline SE & 5 & 3 & & \\
\hline Det. Rate & $1.3(0.5-2.0) \mathrm{NS}$ & $0.7(0.4-0.9)$ & 0.3 & 1.8 \\
\hline SE & 0.3 & 0.1 & & \\
\hline \multicolumn{5}{|l|}{ PTA, dB } \\
\hline $\mathrm{n}$ & 11 & 33 & 42 & 41 \\
\hline Onset level & $66(53-80) \quad S$ & $41(31-52)$ & 65 & 46 \\
\hline SE & 6 & 5 & & \\
\hline Det. gradient & $1.0(0.5-1.6) \mathrm{S}$ & $0.4(0.3-0.6)$ & 0.5 & 1.2 \\
\hline SE & 0.3 & 0.1 & & \\
\hline
\end{tabular}

Onset age (years), onset level $(\mathrm{dB})$, deterioration (det.) rate (\% per year) and deterioration gradient (\% per $\mathrm{dB}$ ) are shown for the original and the present DFNA5 families. Age and PTA indicate $95 \%$ confidence intervals. $\mathrm{n}=$ number of cases, $\mathrm{SE}=$ standard error, $\mathrm{S}=$ significantly different, $\mathrm{NS}=$ not significantly different. The data of the present family shown in the table were derived from the linear curve that approximates the most relevant part of the continuous curve. The two columns on the right show reference data of DFNA2 and DFNA9.

\section{A}

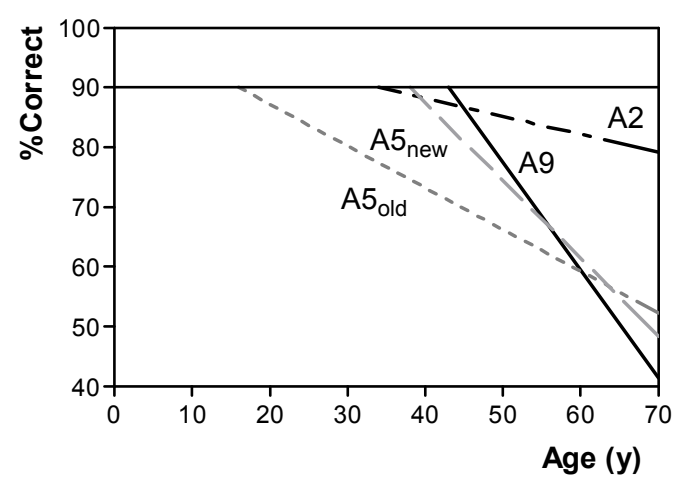

B

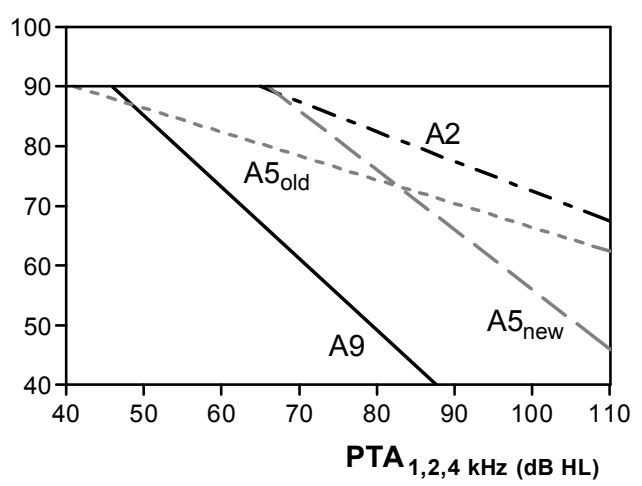

Figure 6. Comparison of performance versus age (A) and performance versus impairment level (B) plots pertaining to speech recognition scores of the present (A5new, dashed line) and the original (A5old, dotted line) DFNA5 patients. Linear plots $\left(X>X_{90}\right)$ for the scores of DFNA2 (A2) and DFNA9 (A9) patients are included for comparison (see text).

The present findings were compared to and tested against the previously reported results for the original DFNA5 family. ${ }^{18}$ It appeared that onset age, onset level and deterioration gradient were significantly higher in the present family (Table 1, Figure 6). The deterioration rate of the present family, although substantially higher, did not differ significantly from the one derived for the original DFNA5 
family. Figure 6 demonstrates that at an age of $<65$ years, our patients had better recognition scores than the patients from the original family, which could also be explained by their more favourable thresholds in the speech frequencies in the first four decades of life (Figure 4). Patients from the two DFNA5 families tended to develop similar poor phoneme scores at an advanced age (Figure 6A) and around a PTA level of about $85 \mathrm{~dB}$ at a score of about 75\% (Figure 6B). Remarkably, Figure 6B suggests the scores to diverge at higher PTA levels, with relatively better performance in the original DFNA5 family. ${ }^{18}$

\section{Vestibular function tests and computerized tomography}

Although no vestibular problems appeared to be present in this family, vestibular function was tested in six cases. Electronystagmography was normal in five cases (IV:22, IV:25, IV:29, V:28 and V:47) and revealed a hyperactive vestibulo-ocular reflex in one individual (V:41). Computerized tomography (case IV:22) showed a normal temporal bone configuration.

\section{Linkage analysis}

Due to the similarity of the type of hearing loss in the present family as compared to that in the DFNA5 family described previously, linkage studies were initiated with markers flanking the DFNA5 gene. The markers D7S629 and D7S673 are located telomeric to the DFNA5 gene at 7p15.3 and D7S2493 and D7S529 are derived from the region centromeric to the gene. All markers are located within a distance of $2.5 \mathrm{cM}$ from the gene according to the Généthon genetic map. ${ }^{26}$ All affected individuals were included in the linkage analysis, as well as non-affected individuals from the age of 30 years onwards. As already suggested by the type of hearing loss, there was cosegregation of the disorder and a specific haplotype in the DFNA5 interval (Figure 1). A maximum lod score of 6.82 was calculated for marker D7S673. The maximum lod score for markers D7S629, D7S2493 and D7S529 were 3.85, 2.34 and 5.09 respectively.

\section{Mutation analysis}

For two affected family members the coding region and the exon-intron boundaries of the DFNA5 gene were analysed for the presence of a mutation by DNA sequencing. When compared to the published sequence, two changes were found. The first change was a $C$ to $G$ transversion in the splice acceptor site of intron 7 at position -6 , which was heterozygously present. The second change was a c. $1200 \mathrm{G}>\mathrm{A}$ transition in exon 9 (1257 G>A), ${ }^{24}$ which was homozygously present. This was a silent mutation that was also seen homozygously in patients from the 
original DFNA5 family and the majority of the control individuals. The $\mathrm{C}$ to $\mathrm{G}$ transversion created an $R s a \mathrm{I}$ restriction site. The cosegregation of the mutation in the family could therefore be shown by restriction digestion of a 265-bp PCR fragment encompassing the mutated splice site, resulting in two fragments of 226 $\mathrm{bp}$ and $39 \mathrm{bp}$ in length (Figure 7). The mutation was not present in 100 control individuals.

No deletions in any part of the gene could be detected by probing a Southern blot containing genomic DNA of two patients (III:15 and IV:1) and two control individuals with cDNA representing the entire protein-coding region (data not shown).

A

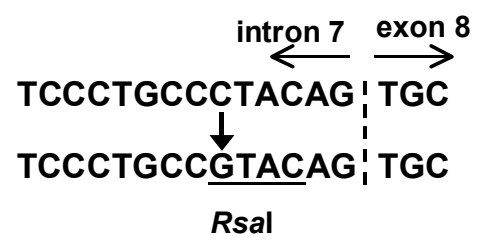

B

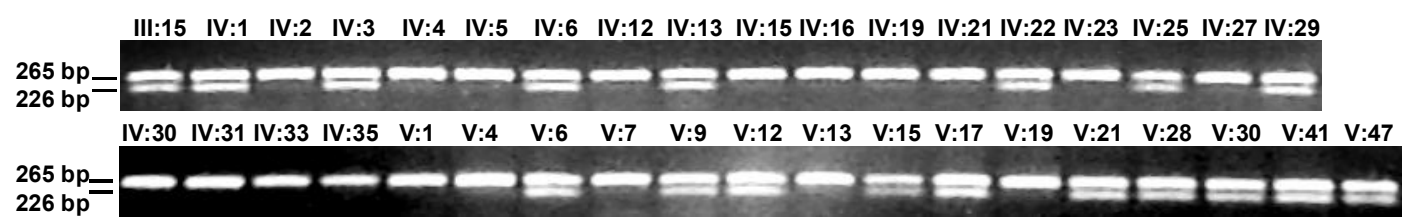

Figure 7. Restriction analysis. A. Sequence of the boundary of intron 7 and exon 8 of the DFNA5 gene, showing that the mutation creates an RsaI restriction site. B. Restriction digestion with $R s a \mathrm{I}$ in 18 affected and 19 non-affected family members. The $265 \mathrm{bp}$ fragment represents the wild type allele, the 226-bp fragment the mutant allele.

\section{Effect of the splice site mutation on splicing efficiency}

Although the detected mutation in the splice acceptor site of intron 7 was not predicted $^{27}$ to lead to a reduced splicing efficiency (86.25 versus 85.00$)$, mRNA derived from cultured lymphoblastoid cells was analysed for the presence of alternative splicing products. RT-PCR with primers located in exon 7 and exon 9 resulted in a fragment of $152 \mathrm{bp}$, which indicates skipping of exon 8 (Figure 8A). The absence of exon 8 in the fragment was confirmed by sequence analysis. Skipping of exon 8 causes a frame shift at amino acid 330. This leads to 41 aberrant codons followed by a premature stopcodon. The amount of the short PCR product found in this study was lower than the amount found in patients from the original DFNA5 family, in which the mutation also affects splicing of intron 7 leading to skipping of exon 8 (Figure 8A). ${ }^{11} \mathrm{~A}$ variable but low amount of the PCR fragment representing the alternatively spliced mRNA was also seen in some control individuals. Therefore, 22 control individuals, two patients of the 
present family and two patients of the original DFNA5 family were compared with regard to the ratio between RT-PCR products with and without exon 8 by phospho-imaging (Figure 8B). One control measurement was detected as an outlier because the background signal was higher than the signal from the alternatively spliced mRNA resulting in a negative measured ratio. Therefore this measurement was excluded from the analysis.

For the remaining control measurements, the mean was 0.034 and SD 0.024 . Ninety-five \% tolerance limits, according to $t$ statistics ranged from -0.017 to 0.084. All DFNA5 patients had values above the upper tolerance limit. Skipping of exon 8 was therefore statistically significantly more frequent in patients of the present and the original family as compared to control individuals.

A
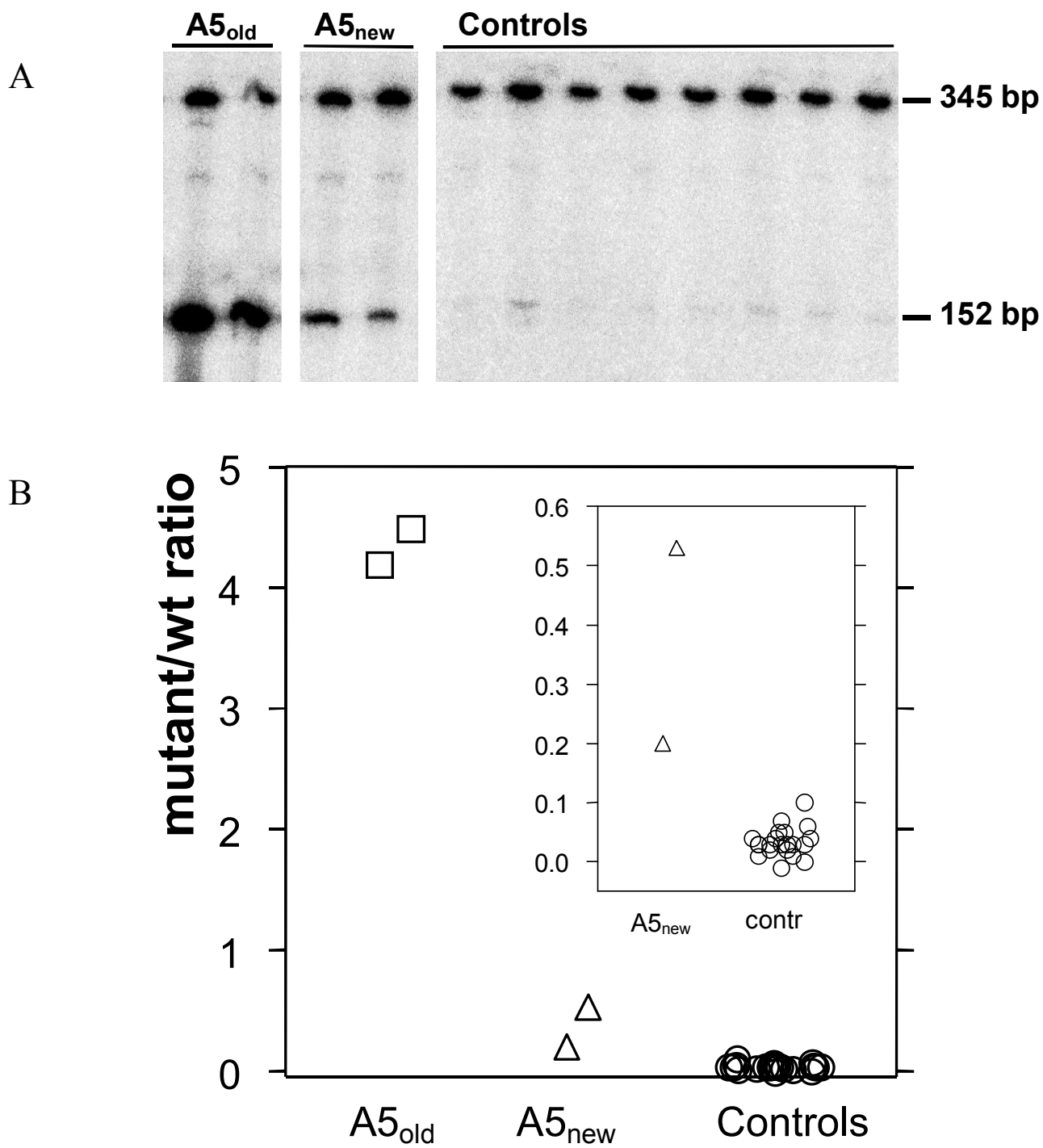

Figure 8. (A) Aberrant splicing of exon 8 of the DFNA5 gene. Two affected persons from the original DFNA5 family (A5old), two affected persons from the present DFNA5 family (A5new) and 8 control individuals (controls) are shown. The wild type splice product is 345 bp long; the aberrant splice product in which exon 8 is skipped is $152 \mathrm{bp}$ long. (B) Ratios of mutant versus wild type (wt) cDNA of two patients of the present family (A5new), two 
patients of the original DFNA5 family (A5old) and 22 control individuals (controls). The inset shows part of the data on a different scale.

Student's $t$ test detected a significant difference $(\mathrm{P}=0.003)$ between the original DFNA5 patients (mean 4.34) and the present DFNA5 patients (mean 0.365). Consequently, splicing in the patients of the present family was less severely disturbed than in the original DFNA5 family. The presence of the C-to-G transversion in the splice acceptor site of intron 7 at position -6 was excluded in the 22 normal hearing control individuals by restriction digestion with RsaI and DNA sequencing.

To exclude that the difference in the amount of the long and the short splice product was a result of preferential degradation of the short splice form by NMD, we used the chemical cycloheximide to inhibit NMD in lymphoblastoid cell cultures of two affected individuals from the present family. We found a 1.36-fold increase of the average ratio when cycloheximide was added (data not shown). Because the amounts of long as well as short splice product were increased after treatment, 1.33- and 1.80-fold respectively, the increased ratio could only be attributed to the presence of an increased amount of short splice product.

To exclude additional intronic mutations that affect correct splicing of the DFNA5 mRNA, RT-PCR was performed with mRNA derived from cultured lymphoblastoid cells of two patients and two control individuals. Six primer combinations were used, which amplify overlapping cDNA fragments, covering the entire coding region. There were no indications for additional alternative splicing (data not shown).

\section{Discussion}

We report the second family with a mutation in the DFNA5 gene associated with hearing loss. Noticeable is the fact that the mutation in both families influences the splicing process resulting in skipping of exon 8 , however to a different extent. The relatively fast elucidation of the molecular defect in this second DFNA5 family, based on a comparison of the type of hearing loss with that of a genetically elucidated type, illustrates the value of a detailed analysis of hearing loss in families.

Sensorineural hearing impairment in the present family was non-syndromic and symmetric with progression of $0.7-1.8 \mathrm{~dB} /$ year, depending on the frequency. Progression was fairly constant, in contrast with the original family, in which there was more rapid progression in the first four decades than at more advanced ages, especially in the high frequencies (about $1 \mathrm{~dB} /$ year at $0.25-1 \mathrm{kHz}$ and $1-4$ $\mathrm{dB} /$ year at 2-8 kHz). ${ }^{12}$ The ARTA, however, were not significantly different. A 
large variation in audiogram types was found, which was also reported in the original family. There was more severe hearing loss in the younger individuals than was predicted following the present procedures.

Speech recognition scores were relatively good with an onset age of 37 years and deterioration rate of $1.3 \%$ per year. Recognition scores decreased by $1.0 \%$ per $\mathrm{dB}$ threshold increase from a mean PTA $1,2,4 \mathrm{kHz}$ of $63 \mathrm{~dB}$ onwards. The difference in speech recognition between both families might be explained by the more favourable thresholds in the speech frequencies in the first four decades of life, demonstrated by the ARTA of the present family.

Table 1 and Figure 6 include data derived from previous reports on DFNA2 and DFNA9. ${ }^{16}$ Straight lines were fitted for $X>X_{90}$ to approximate the regression curves. The original DFNA5 patients tended to show similar phoneme scores as the DFNA9 patients, only at a more advanced age. The present DFNA5 patients showed this similarity already from a younger age onwards. At a given level of hearing impairment, DFNA5 patients of both families generally showed recognition scores that were better than those of DFNA9 patients, but worse than those of DFNA2 patients. As previously described, ${ }^{16}$ the high-frequency sensorineural hearing impairment of DFNA2 may be attributed to lack of expression of potassium channels, especially in the inner hair cells of the lower turns in the cochlea. Relatively better speech recognition in DFNA2 was explained by relative sparing of outer hair cell function in this region preserving pre-amplification and fine-tuning mechanisms. The relatively good speech recognition performance in DFNA5 patients might be explained in a similar way.

The similarities in the type of hearing loss and a comparable effect of the mutations, namely skipping of exon 8 in both the present and the original DFNA5 family, indicate that the mutation in the present family indeed is the diseasecausing genetic defect. Also, despite one silent mutation, no other mutations were found in the DFNA5 gene in the present family. Although the transcript without exon 8 was also seen in control individuals, the relative amount was statistically significantly higher in the two patients from the present family than in the control individuals. Also, we have to note that the effect of the mutation on splicing might be different in the inner ear compared to lymphoblastoid cell lines. The $1200 \mathrm{G}>\mathrm{A}$ polymorphism has no major influence on the splicing efficiency of exon 8 , because the disease-causing mutation in the present and the original family co-segregates with an A at position 1200. Also, the majority of the controls were homozygous A. Even though our results show that the ratio between the long and short splice form increases after treatment with cycloheximide, the lower amount of short splice product as compared to the amount of long splice product in the present 
family remains, indicating that this difference is not a result of NMD. Also, the low amount of short mRNA in the present family as compared to the previously described family cannot be explained by a higher rate of NMD in the present family.

The relatively low amount of the short transcript in the present family suggests that not haploinsufficiency but a dominant negative effect of the mutant protein causes the hearing loss, which might be attributed to the relatively large stretch of 41 aberrant amino acids. Since the alternative splicing product is also seen in a variable but lower ratio to the normal mRNA in controls, there might be a critical threshold for the aberrant protein above which it becomes pathogenic. This is underlined by the fact that the mutations in the only two DFNA5 families known so far have the same effect on the mRNA and the putative protein but affect a different amount of the transcript. Unfortunately, nothing is known about either the wild type or the short protein. Both proteins have very different $C$ termini and neither have similarity with any other protein or protein domain. Whether the differences in speech recognition between the two families are due to the different ratios of normal versus short protein remains to be elucidated. One might think about discrepancies in pathogenic thresholds in different parts of the cochlea. Alternatively, modifier genes might be involved. Findings in additional families with a mutation in the DFNA5 gene may elucidate whether other mutations in the gene also lead to a similar type of hearing impairment or that only the putative short protein causes hearing loss.

The function of the DFNA5 protein is still unknown, although there are some clues indicating that DFNA5, also designated ICERE-1, might be involved in the regulation of apoptosis. Recently, Lage et al. reported that decreased DFNA5 mRNA levels are associated with a decrease in apoptotic events after exposure to the drug etoposide. ${ }^{28}$ It appeared that increased DFNA5 mRNA levels are associated with increased cellular disposition to programmed cell death mediated by activation of caspase 3. However, it has not yet been proven that there is a direct effect of DFNA5 on apoptosis. Interestingly, apoptosis is important in the development of the inner ear predominantly during the embryonic and early postnatal development of the cochlea. ${ }^{29-32}$ Two studies of caspase 3 knockout mice have shown that caspase 3 deficiency results in severe hearing loss. ${ }^{33,34}$ Hyperplasia of supporting cells and degeneration of sensory hair cells and cochlear neurons are seen in the inner ear of these mice. ${ }^{33,34}$ In 5-week-old homozygous caspase 3 knockout mice extensive loss of hair cells was observed in the basal and middle turns of the cochlea but not in the apical turns. Degeneration of the hair cells begins early, progressively worsens with age and spreads from 
the basal turns towards the apical turns. These findings might explain why DFNA5 is a progressive form of hearing loss that affects the higher and middle frequencies earlier and more severely than the lower frequencies. The ongoing characterization of mice in which the mutation in the original DFNA5 family is mimicked will elucidate whether the cochlear defects are similar to those in the caspase-3-deficient mice and thus whether DFNA5 might have a function in apoptosis in the inner ear. ${ }^{35}$

\section{References}

1. Van Camp G, Smith RJH. Hereditary Hearing Loss Homepage. World Wide Web URL: http://www.uia.ac.be/dnalab/hhh.

2. Huygen PLM, Pennings RJE, Cremers CWRJ. Characterizing and distinguishing progressive phenotypes in nonsyndromic autosomal dominant hearing impairment. Audiol Med 2003;1;37-46.

3. Huizing EH, van Bolhuis AH, Odenthal DW. Onderzoekingen over progressieve hereditaire perceptiedoofheid bij een uit 355 leden bestaande familie. Ned Tijdschr Geneesk 1965;109:499-503.

4. Huizing EH, van Bolhuis AH, Odenthal DW. Studies on progressive hereditary perceptive deafness in a family of 335 members. I. Genetical and general audiologic results. Acta Otolaryngol 1966;61:35-41.

5. Huizing EH, van Bolhuis AH, Odenthal DW. Studies on progressive hereditary perceptive deafness in a family of 335 members. II. Characteristic pattern of hearing deterioration. Acta Otolaryngol 1966;61:161-167.

6. Huizing EH, Odenthal DW, van Bolhuis AH. Results of further studies on progressive hereditary sensorineural hearing loss. Audiology 1972;12:261-263.

7. Huizing EH, van den Wijngaart WSIM, Verschuure J. A follow-up study in a family with dominant progressive inner ear deafness. Acta Otolaryngol 1983;95:620-626.

8. van den Wijngaart WSIM, Verschuure J, Brocaar MP, Huizing EH. Follow-up study in a family with dominant progressive hereditary sensorineural hearing impairment. I. Analysis of hearing deterioration. Audiology 1985;24:233-240.

9. van den Wijngaart WSIM, Huizing EH, Niermeijer MF, Verschuure J, Brocaar MP, Blom W. Follow-up study in a family with dominant progressive hereditary sensorineural hearing impairment. II. Clinical aspects. Audiology 1985;24:336-342.

10. Van Camp G, Coucke P, Balemans W, van Velsen D, van de Bilt C, Van Laer L, Smith RJH, Fukushima K, Padberg GW, Frants RR, Van de Heyning P, Smith SD, Huizing EH, Willems PJ. Localisation of a gene for non-syndromic hearing loss (DFNA5) to chromosome 7p15. Hum Mol Genet 1995;4:2159-2163.

11. Van Laer L, Huizing EH, Verstreken M, van Zuijlen D, Wauters JG, Bossuyt PJ, Van de Heyning P, McGuirt WT, Smith RJH, Willems PJ, Legan PK, Richardson GP, Van Camp G. Nonsyndromic hearing impairment is associated with a mutation in DFNA5. Nat Genet 1998;20:194-197.

12. De Leenheer EMR, van Zuijlen DA, Van Laer L, Van Camp G, Huygen PLM, Huizing EH, Cremers CWRJ. Clinical features of DFNA5. In: Cremers CWRJ, Smith RJH (eds). Genetic hearing impairment. Its clinical presentations. Advances in Oto-Rhino-Laryngol. Basel: Karger 2002, vol 61. pp 53-59.

13. ISO 389. Acoustics. Standard reference zero for the calibration of pure tone air conduction audiometers. Geneva: International Organisation for Standardization, 1985. 
14. ISO 8253-1.Acoustics. Audiometric test methods, I: basic pure tone air and bone conduction threshold audiometry. Geneva: International Organisation for Standardization, 1989.

15. ISO 7029. Acoustics. Threshold as hearing by air conduction as a function of age and sex for otologically normal persons. Geneva: International Organisation for Standardization, 1984.

16. Bom SJH, De Leenheer EMR, Lemaire FX, Kemperman MH, Verhagen WIM, Marres HAM, Kunst HPM, Ensink RJH, Bosman AJ, Van Camp G, Cremers FPM, Huygen PLM. Speech recognition scores related to age and degree of hearing impairment in DFNA2/KCNQ4 and DFNA9/COCH. Arch Otolaryngol Head Neck Surg 2001;127:1045-1048.

17. Bom SJH, Kemperman MH, Huygen PLM, Luijendijk MWJ, Cremers CWRJ. Cross-sectional analysis of hearing threshold in relation to age in a large family with cochleovestibular impairment thoroughly genotyped for DFNA9/COCH. Ann Otol Rhinol Laryngol 2003;112:280-286.

18. De Leenheer EMR, van Zuijlen DA, Van Laer L, Van Camp G, Huygen PLM, Huizing EH, Cremers CWRJ. Further delineation of the DFNA5 phenotype. Results of speech recognition tests. Ann Otol Rhinol Laryngol 2002;111:639-641.

19. Miller S, Dykes D, Polensky H. A simple salting out procedure for extracting DNA from human nucleated cells. Nucleic Acids Res 1988;16:1215.

20. Kremer H, Kuyt L, van den Helm B, van Reen M, Leunissen JA, Hamel BC, Jansen C, Mariman EC, Frants RR, Padberg GW. Localization of a gene for Möbius syndrome to chromosome 3q by linkage analysis in a Dutch family. Hum Mol Genet 1996;5:1367-1371.

21. Lathrop G, Lalouel J. Easy calculations of lod scores and genetic risks on small computers. Am J Hum Genet 1984;36:460-465.

22. Lathrop G, Lalouel J, Julier C, Ott J. Strategies for multilocus linkage analysis in humans. Proc Natl Acad Sci USA 1984;81:3443-3446.

23. Lathrop G, Lalouel J, White R. Construction of human linkage maps: likelihood calculations for multilocus linkage analysis. Genet Epidemiol 1986;3:39-52.

24. Van Laer L, DeStefano AL, Myers RH, Flothman K, Thys S, Fransen E, Gates GA, Van Camp G, Baldwin CT. Is DFNA5 a susceptibility gene for age-related hearing impairment? Eur J Hum Gen 2002;10:883-886.

25. Thompson DA, Weigel RJ. Characterization of a gene that is inversely correlated with estrogen receptor expression (ICERE-1) in breast carcinomas. Eur J Biochem 1998;252:169177.

26. Dib C, Faure S, Fizames C, Samson D, Drouot N. Vignal A, Millasseau P, Marc S, Hazan J, Seboun E, Lathrop M, Gyapay G, Morissette J, Weissenbach J. A comprehensive genetic map of the human genome based on 5,264 microsatellites. Nature 1996;380:152-154.

27. Shapiro MB, Senapathy P. RNA splice junctions of different classes of eukaryotes: sequence statistics and functional implications in gene expression. Nucleic Acids Res 1987;15:71557174 .

28. Lage H, Helmbach H, Grottke C, Dietel M, Schadendorf D. DFNA5 (ICERE-1) contributes to acquired etoposide resistance in melanoma cells. FEBS Letters 2001;494:54-59.

29. Jókay I, Soós G, Répássy G, Dezsõ B. Apoptosis in the human inner ear. Detection by in situ end-labeling of fragmented DNA and correlation with other markers. Hearing Res 1998;117:131-139.

30. Nishizaki K, Anniko M, Orita Y, Karita K, Masuda Y, Yosino T. Programmed cell death in the developing epithelium of the mouse inner ear. Acta Otolaryngol 1998; 18: 96-100.

31. Nishikori T, Hatta T, Kawauchi H, Otani H. Apoptosis during inner ear development in human and mouse embryos: An analysis by computer-assisted three dimensional reconstruction. Anat Embryol 1999;200:19-26.

32. Nikolic P, Järlebark LE, Billett TE, Thorne PR. Apoptosis in the developing rat cochlea and its related structures. Devel Brain Res 2000;119:75-83. 
Phenotype and genotype of a novel DFNA5 family

33. Morishita H, Makishima T, Kaneko C, Lee YS, Segil N, Takahashi K, Kuraoka A, Nakagawa T, Nabekura J, Nakayama K, Nakayama KI. Deafness due to degeneration of cochlear neurons in caspase-3-deficient mice. Biochem Biophys Res Comm 2001;284:142-149.

34. Takahashi K, Kamiya K, Urase K, Suga M, Takizawa T, Mori H, Yoshikawa Y, Ichimura K, Kuida K, Momoi T. Caspase-3-deficiency induces hyperplasia of supporting cells and degeneration of sensory cells resulting in hearing loss. Brain Res 2001;894:359-367.

35. Van Laer L, Pfister M, Thys S, Umans L, Serneels L, Kooy F, Timmermans JP, Van Leuven F, Van Camp G. The DFNA5 mouse: the first analysis of the phenotype. Acta Otorhinolaryngol Belg 2002;56:267 (Abstract). 


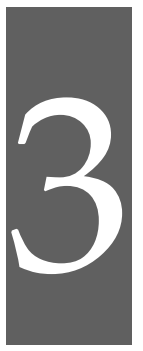

DFNA9 



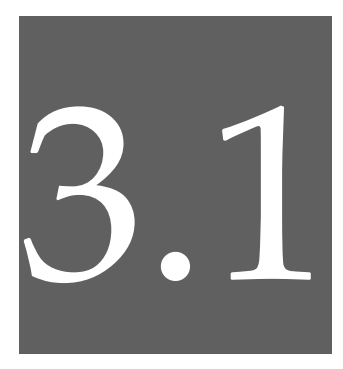

\title{
Vestibular deterioration precedes hearing deterioration in the P51S $\mathrm{COCH}$ mutation (DFN9): an analysis in 74 mutation carriers
}

\author{
A.M.L.C. Bischoff \\ P.L.M. Huygen \\ M.H. Kemperman \\ R.J.E. Pennings \\ S.J.H. Bom \\ W.I.M. Verhagen \\ R.J.C. Admiraal \\ H. Kremer \\ C.W.R.J. Cremers
}





\begin{abstract}
Objectives: To analyse cochleovestibular impairment features in P51S COCH mutation carriers $(n=22)$ in a new, large Dutch family and to compare the results to those obtained in previously identified similar mutation carriers $(n=52)$. To evaluate age-related features between progressive hearing and vestibular impairment of all mutation carriers $(n=74)$.

Study Design: Family study.

Methods: Regression analysis was performed in relation to age to outline the development of hearing thresholds, speech recognition scores and vestibuloocular reflex time constant as the key vestibular response parameter.

Results: Pure tone thresholds, phoneme recognition scores and vestibular responses of the mutation carriers in the new family were essentially similar to those previously established in all other mutation carriers. Hearing started to deteriorate in all mutation carriers from 43 years of age onwards, whereas deterioration of vestibular function started from the age of 34 .

Conclusion: Vestibular impairment starts earlier, progresses more rapidly, and eventually, is more complete than hearing impairment in P51S COCH mutation carriers.
\end{abstract}

\title{
Introduction
}

The first DFNA9 family with progressive cochleovestibular dysfunction developing after the age of 40, was reported by Verhagen et al. ${ }^{1}$ Eight years later, genetic linkage was found for several American families to a locus, designated DFNA9, on chromosome 14q12-13. ${ }^{2}$ The COCH gene was isolated from a human foetal cochlear cDNA library and high levels of expression were found in the cochlear and vestibular labyrinth. ${ }^{3}$ So far, five different mutations have been identified: three American (V66G, G88E, W117R), a Dutch/Belgian (P51S) and most recently, an Australian I109N missense mutation. ${ }^{4-6}$ Whereas hearing impairment started in the second to third decade in the Australian and American V66G and W117R mutations, ${ }^{4,6}$ it developed from the fifth decade onwards in the other mutations. In 1991, glucosaminoglycan-like depositions were reported for the first time in the cochlea of DFNA9 patients, which have been suggested to cause the progressive cochleovestibular dysfunction. ${ }^{7}$ An alternative hypothesis is that $\mathrm{COCH}$ plays a role in endolymph homeostasis. ${ }^{8}$ DFNA9 and DFNA119 are presently the only types of non-syndromic autosomal dominant hearing impairment with concomitant vestibular impairment. 
This report is an account of an in-depth analysis of cochleovestibular impairment features in P51S COCH mutation carriers in a new large Dutch family, combined with previously identified mutation carriers, ${ }^{1,10-17}$ as well as some additional newly identified ones. Clinical results of several Dutch/Belgian P51S families have previously been described, 7,10,13,15,18 including follow-up findings ${ }^{14,16,17}$ and a comprehensive cross-sectional analysis of pure tone thresholds related to age. ${ }^{12}$ These studies only included limited longitudinal observations of cochleovestibular features. Recently, we identified a new large P51S COCH family with many carriers, of which extensive longitudinal data were available. Ophthalmologic examination in this family disclosed the presence of a peculiar pattern of corneal striae, which is the subject of a separate paper. ${ }^{19}$ Therefore, whether the cochleovestibular findings in this family differed from those obtained in previous studies on Dutch P51S families was checked before any data were pooled for further analysis.

Previous cross-sectional analyses of speech recognition scores in P51S COCH mutation carriers were limited to retrospectively collected incidental measurements in patients from several different families. ${ }^{11}$ In the present family, it was possible to perform speech audiometry prospectively in all mutation carriers with hearing impairment symptoms.

Vestibular examination was also performed in most of these affected family members, which allowed for an intrafamilial quantitative cross-sectional analysis of vestibular response parameters in relation to age. This analysis could be extended to a suprafamilial one by adding vestibular test results obtained in all our other P51S COCH mutation carriers. Finally, it was possible to compare between estimates of onset age and rate of hearing deterioration as well as vestibular dysfunction from all simultaneously collected cross-sectional vestibular and hearing impairment data.

\section{Patients and Methods}

Forty-four family members of a new Dutch family harbouring the P51S COCH trait participated in this study. Signed informed consent was obtained from all participants. Medical history was taken, with special attention paid to vestibular, ocular and hearing impairment symptoms. Non-hereditary causes of hearing impairment were excluded. All individuals underwent otoscopic examination and pure tone audiometry. Speech audiometry and vestibular function tests were performed in clinically affected persons. Guided by the clinical findings, $\mathrm{COCH}$ 
mutation analysis was performed in all of them, which confirmed the presence of the disease-causing P51S mutation.

Four affected family members underwent brainstem auditory evoked potential (BAEP) examination. One person previously underwent computerized tomography and in four persons magnetic resonance imaging of the temporal bones was performed.

\section{Audiometry and data analysis}

Pure tone and speech audiometry were performed in a sound treated room, the former according to the norms of the International Standard Organization (ISO). ${ }^{20,21}$ Threshold was fixed at an arbitrary value of $130 \mathrm{~dB}$ hearing level for outof-scale measurements. The individual $95^{\text {th }}$ percentile threshold values of presbyacusis $\left(\mathrm{P}_{95}\right)$ in relation to sex and age were derived for each frequency, using the ISO 7029 method. ${ }^{22}$ Subjects were considered affected if the best hearing ear showed thresholds beyond the $P_{95}$. Longitudinal regression analysis was performed of binaural mean threshold values on age in clinically affected persons with three or more consecutive measurements at ages of less than 70 years and a follow-up interval of more than three years. The regression coefficient (slope) was called annual threshold deterioration (ATD), expressed in $\mathrm{dB}$ per year. Progression was significant if the 95\% confidence interval of the ATD did not include zero. An analysis similar to analysis of covariance (ANCOVA, Prism PC version 3; GraphPad, San Diego CA, U.S.A.) was performed to compare between slopes and intercepts of the regression lines, pertaining to individual measurements per frequency. As pooled regression appeared to be inappropriate, the median slope (ATD) and $Y$ intercept were calculated to establish a trend line for each frequency. Because the trend line thus obtained only reflected the longitudinally analysed cases, we checked whether the total numbers of affected persons (i.e. including those who did not undergo longitudinal analysis) covered by data points above and below the trend line were about equal for each separate frequency. The results obtained in the present longitudinal data analysis were compared with the results of a cross-sectional analysis (last-visit thresholds) for 52 other Dutch P51S COCH mutation carriers (see Table 3 for equation). ${ }^{12}$ Those carriers were selected who had simultaneous measurements of pure tone hearing thresholds and vestibulo-ocular reflex (VOR) parameters. In addition, longitudinal measurements (follow-up interval 2-12 years) were available in 10 of these carriers.

Speech audiometry was performed using a standard monosyllabic (consonantvocal-consonant) Dutch word list. Maximum monaural phoneme scores 
(percentage correct recognition) were derived from individual performance versus intensity plots. Cross-sectional recognition scores averaged for both ears were plotted against age and binaural mean pure tone average (PTA1,2,4 $\mathrm{kHz})$ in $\mathrm{dB}$ hearing level. In the performance versus impairment plot, nonlinear regression analysis was used to fit sigmoidal dose-response curves with a variable slope (same equation as included in Table 3). The age of onset and the onset level (i.e. $X_{90}$ ) were defined at a recognition score of $90 \%$ in a performance $(Y)$ versus age or versus impairment $(X)$ plot, respectively. For $X_{90}>X>X_{30}$, a straight line was fitted to simplify the results and to obtain an estimate of local average slope that was called deterioration rate in the score-against-age plot and deterioration gradient in the score-against-PTA $1,2,4 \mathrm{kHz}$ plot. ${ }^{11}$ The results of the present singlefamily cross-sectional data analysis were compared with the results of a previously performed multi-family analysis in P51S COCH mutation carriers. ${ }^{11}$

\section{Vestibular examination and data analysis}

Twenty-two of the affected persons underwent vestibular examination with eyes open in the dark, employing a rotatory chair to perform a $90 \%$ velocity step test in two directions with electronystagmography and computer analysis, as previously described. ${ }^{23}$ The time constant $\mathrm{T}$ (s) was elected to characterize the VOR. As this parameter shows a lognormal distribution, ${ }^{24}$ the geometric mean for both nystagmus directions was used for further analysis. The $90 \%\left(\mathrm{P}_{5}-\mathrm{P}_{95}\right)$ confidence interval for this parameter was 13-23 s. Table 1 shows the classification by $\mathrm{T}$ value used to categorize the VOR findings obtained with the velocity step test.

Table 1. Classification of VOR findings in velocity-step test according to the value of the time constant $(\mathrm{T})$.

\begin{tabular}{|l|l|}
\hline $\mathrm{T}(\mathrm{s})$ & Classification \\
\hline$>23$ & Hyperreflexia \\
\hline $13-23$ & Normal \\
\hline $5-12$ & Hyporeflexia \\
\hline$<5$ & Severe hyporeflexia, close to areflexia \\
\hline 0 & Areflexia \\
\hline
\end{tabular}

Hyporeflexia with an ultrashort time constant $(\mathrm{T}<5 \mathrm{~s})$ was labelled as a separate category of severe hyporeflexia, that is close to areflexia, because primate afferent vestibular neuron recordings have shown that time constants generally are not below five seconds. ${ }^{25}$ In addition, nystagmic rotatory responses exhibiting shorter 
time constants have been suggested to be of extravestibular, presumably somatosensory, origin. ${ }^{15,26}$ An arbitrary zero $(\mathrm{T}=0)$ was assigned to rotatory responses showing no or just a few nystagmus beats. For the sake of comparison, as well as for potential extension of the present data, we retrieved similar last-visit data from the 52 additional mutation carriers specified above. Nonlinear crosssectional regression analysis of $\mathrm{T}$ on age was performed for this combined group and the present group of mutation carriers, separately. Student's $t$ test was used to compare between the fitted parameter values in both groups, including Welch's correction if Bartlett's test identified unequal variances. The level of significance used in all tests was $\mathrm{p}=0.05$.

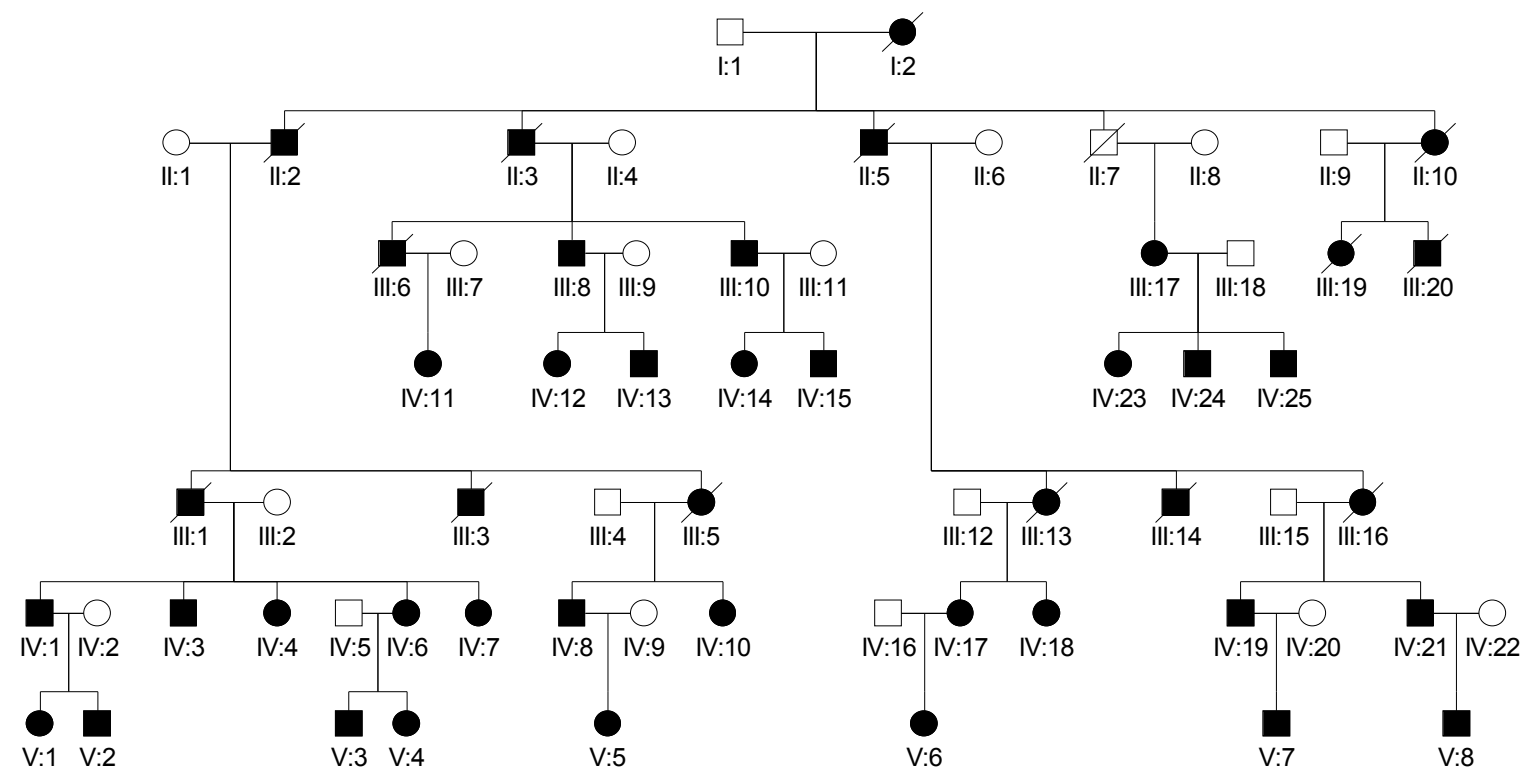

Figure 1. Part of the pedigree of a new Dutch family with the P51S COCH trait. Only affected participants are included, together with deceased affected family members. Filled symbols indicate mutation carriers, open symbols non-carriers. Squares represent men, circles women. Please note that generation lines of different lengths have been used to compact the figure; generation is included in symbol legend.

\section{Results}

\section{General findings and hearing impairment symptoms}

The pedigree of the new family (Figure 1; modified for reasons of privacy) comprised 44 affected family members, 30 of whom were alive and participated in this study. By history, person II:7 did not have profound cochleovestibular impairment when he died at 54 years of age. Otoscopic examination was normal, except in one case (IV:25), in which a granulomatous thickened eardrum (myringitis granulomatosa) was noted. One patient (III:8) had received a cochlear 
implant at age 70 and only the pre-implant data were used for the present analysis. The mean onset of self-reported hearing impairment symptoms was 39 years (range: 18-51 years). Pure tone audiometry indicated that 20 family members were affected. Asymmetry and variability in progression were also noted in this family and were fairly similar to previous findings in other P51S families. ${ }^{11,17,27}$ Tinnitus was mentioned by nine of the present mutation carriers (Table 2).

Table 2. VOR findings in 22 examined mutation carriers of the present family shown together with age, binaural mean hearing level and presence or absence of tinnitus

\begin{tabular}{|c|c|c|c|c|}
\hline $\begin{array}{l}\text { Person } \\
\text { nr. }\end{array}$ & $\begin{array}{l}\text { Age } \\
\text { (years) }\end{array}$ & $\begin{array}{l}\text { Vestibular function: } \\
\text { VOR }\end{array}$ & $\begin{array}{l}\mathrm{PTA}_{1,2,4 \mathrm{kHz}} \\
(\mathrm{dB} \mathrm{HL})\end{array}$ & Tinnitus \\
\hline$V: 3$ & 24 & Hyperreflexia & 2 & No \\
\hline $\mathrm{V}: 7$ & 25 & Normal & 13 & No \\
\hline $\mathrm{V}: 1$ & 31 & Normal & 2 & No \\
\hline IV:11 & 41 & Severe hyporeflexia & 51 & Yes \\
\hline IV:25 & 41 & Severe hyporeflexia & 26 & No \\
\hline IV:13 & 42 & Severe hyporeflexia & 4 & No \\
\hline$V: 5$ & 43 & Normal & 2 & No \\
\hline IV:24 & 44 & Severe hyporeflexia & 46 & No \\
\hline IV:23 & 48 & Hyporeflexia & 34 & No \\
\hline IV:7 & 50 & Areflexia & 38 & Yes \\
\hline IV:18 & 50 & Hyporeflexia & 32 & Yes \\
\hline IV:3 & 53 & Areflexia & 52 & Yes \\
\hline IV:6 & 54 & Severe hyporeflexia & 54 & Yes \\
\hline IV:17 & 54 & Areflexia & 64 & No \\
\hline IV:4 & 56 & Areflexia & 38 & Yes \\
\hline IV:1 & 57 & Areflexia & 48 & Yes \\
\hline IV:19 & 60 & Areflexia & 69 & No \\
\hline IV:10 & 61 & Areflexia & 64 & Yes \\
\hline III:10 & 61 & Areflexia & 83 & No \\
\hline III:8 & 69 & Areflexia & 128 & No \\
\hline IV:8 & 69 & Areflexia & 85 & No \\
\hline III:17 & 77 & Areflexia & 97 & Yes \\
\hline
\end{tabular}

Figure 2 compares the binaural mean PTA $1,2,4 \mathrm{kHz}$ levels between mutation carriers with and without tinnitus, controlling for the patient's age. The two regression 
lines were not significantly different, which implies that the presence of tinnitus did not clearly depend on the extent of hearing impairment.

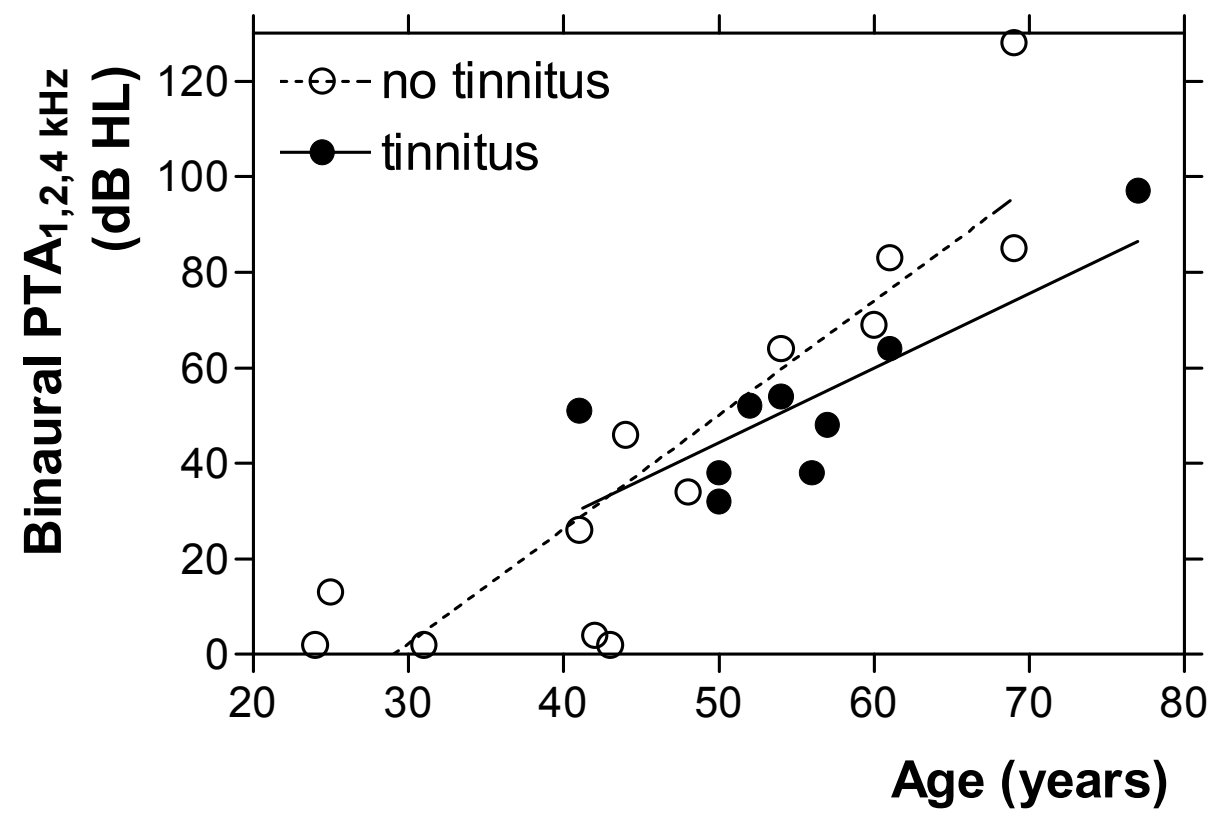

Figure 2. Comparison of binaural mean $\mathrm{PTA}_{1,2,4 \mathrm{kHz}}(\mathrm{dB}$ hearing level) between the present mutation carriers with (filled circles, solid regression line) and without (open circles, dashed regression line) tinnitus, controlling for age (years). The regression lines do not differ significantly (see Methods). HL, hearing level.

BAEP examination showed pathological delay with amplitude attenuation of wave $\mathrm{V}$ on the left side in one person (IV:1). BAEPs were normal in three other affected persons (IV:8, IV:12 and IV:24). Additional computerized tomography scans (III:8) and magnetic resonance imaging analyses (IV:1, IV:3, IV:17 and IV:24) were unremarkable.

\section{Audiologic and vestibular features related to age}

Figure 3 covers all individual threshold measurements obtained from affected members of the present family. The ATD (dotted trend line) ranged from $1.9 \mathrm{~dB}$ per year $(0.5 \mathrm{kHz})$ to $3.3 \mathrm{~dB}$ per year $(8 \mathrm{kHz})$. Extrapolation of the trend line to threshold zero suggested an onset age for progression in the range of 27 to 42 years; however, the validity of such an extrapolation can be doubted. The sigmoidal curves, fitted to the cross-sectional threshold data of the previously identified mutation carriers (see Methods; data points not shown in Figure 3), matched fairly well with the threshold data of the present mutation carriers. We therefore concluded that pooling of cross-sectional hearing threshold data of all mutation carriers was appropriate (see below). 


\section{$0.25 \mathrm{kHz}$}

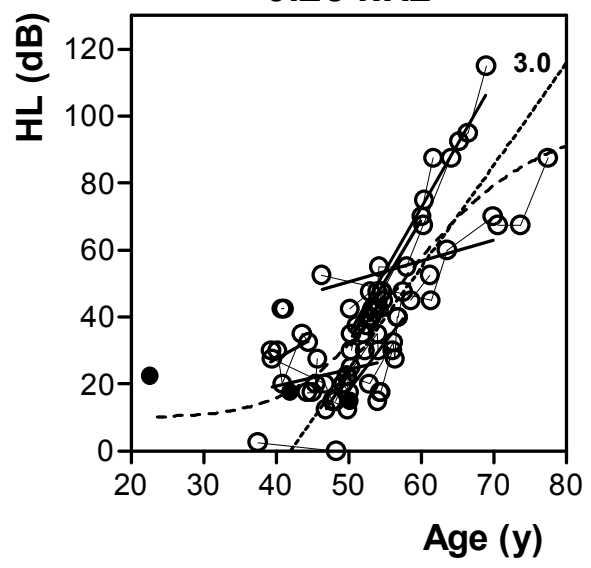

$1 \mathrm{kHz}$
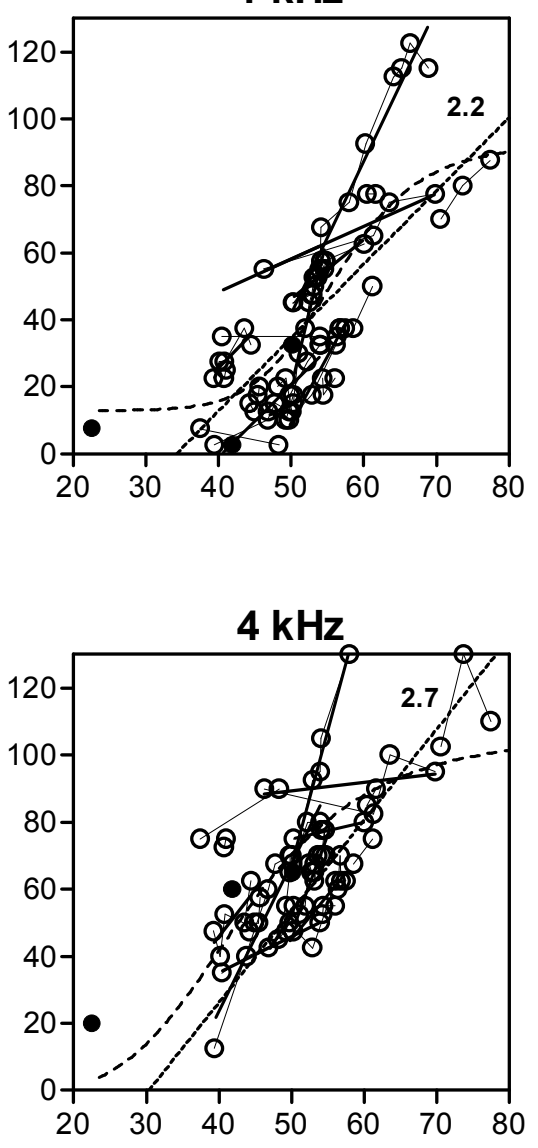

\section{$0.5 \mathrm{kHz}$}

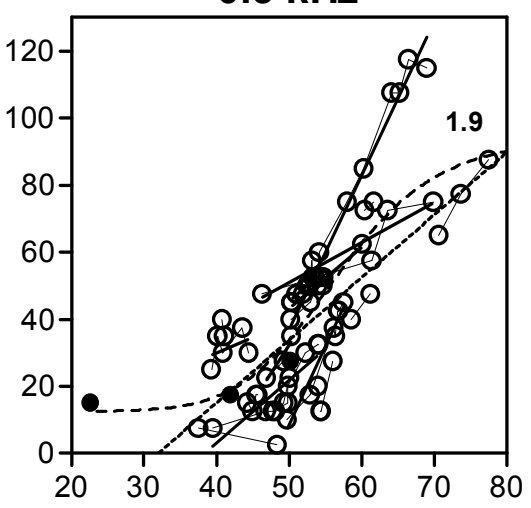

$2 \mathrm{kHz}$

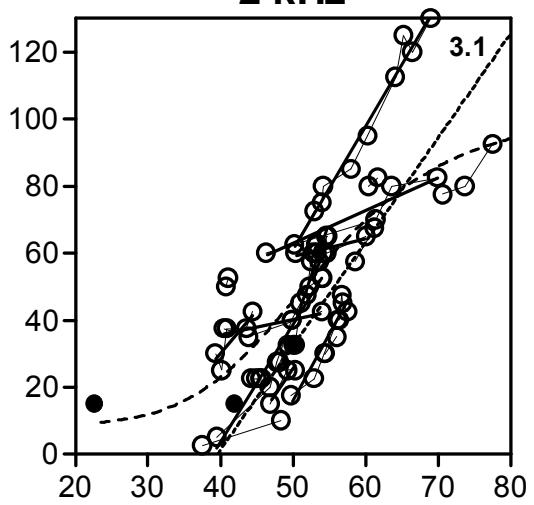

$8 \mathrm{kHz}$

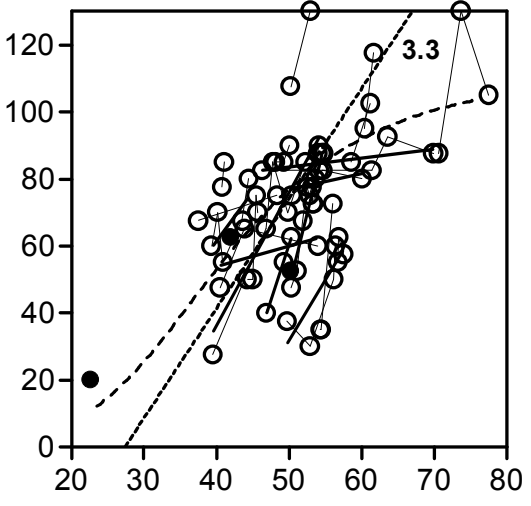

Figure 3. Longitudinal analyses of hearing impairment (binaural mean air conduction threshold) for the present mutation carriers. Threshold data (in $\mathrm{dB}$ hearing level) versus age (in years) for all frequencies combining individual longitudinal data (open circles with connecting hairlines and fitted regression line) and other measurements (open circles without regression lines, or filled circles) of 20 mutation carriers. Bold regression lines indicate significant progression. In each panel, the dotted straight line is the trend line (see Methods). The slope of this line is indicated in bold. The dashed sigmoidal curve in bold was fitted to the cross-sectional threshold-on-age data (data points themselves not shown) of all mutation carriers except those of the present family. HL, hearing level. 

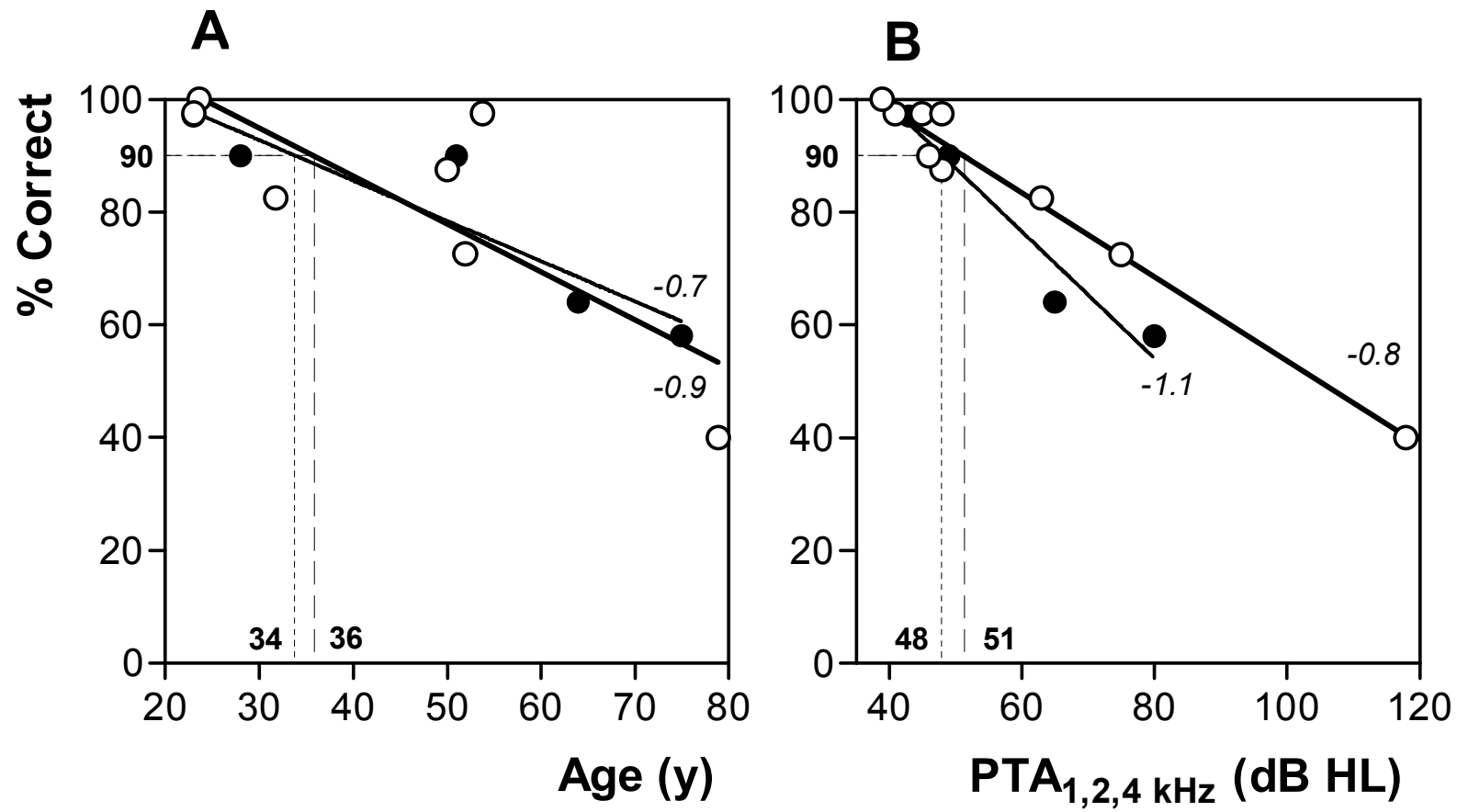

Figure 4. Cross-sectional binaural mean phoneme recognition scores at last visit against age (A) and binaural mean PTA 1,2,4 kHz (B) in the present mutation carriers. The dotted lines with bold number indicate onset age and onset level. The dashed line in part $\mathrm{B}$ indicates "local average" slope for $X_{90}>X>X_{30}$. Bold numbers near the regression line indicate deterioration rate (A) and deterioration gradient (B). HL, hearing level.

The speech recognition scores of the present mutation carriers gradually declined to about $50 \%$ at an age of 66 years (Figure $4 \mathrm{~A}$ ). Onset age $X_{90}$ was 49 years, from which the recognition score deteriorated by $2.3 \%$ per year on average. The $90 \%$ recognition score was found at a $\mathrm{PTA}_{1,2,4} \mathrm{kHz}$ level of $46 \mathrm{~dB}$ (Figure $4 \mathrm{~B}$ ) and deteriorated by $1.2 \%$ per $\mathrm{dB}$ on average for $X_{90}>X>X_{30}$. These values compared fairly well to those previously obtained in P51S mutation carriers from several different families ${ }^{11}$ (original data and fitted parameter values not shown).

Vestibular examination was performed on 22 mutation carriers of the present family, preferably those having cochleovestibular symptoms, aged 24 to 77 years (Table 2). Eighteen persons had experienced characteristic vestibular symptoms. ${ }^{15,17}$ Eleven persons had experienced attacks of vertigo lasting up to half a day, most often not accompanied by nausea. Three persons had paroxysmal vertigo similar to Ménière's disease, with hearing impairment and symptoms of threshold fluctuations. Aural fullness was absent.

In Table 2 the classified results of the vestibular examination are shown sorted by age. Vestibular areflexia was found in 11 of the 13 persons aged 50 years and older. Severe hyporeflexia was found in four of the nine younger individuals at ages 41 to 44 years. Person IV:13 showed severe hyporeflexia with still normal 
audiometry. He recently experienced his first attack of vertigo. Person V:5 was the only one older than 40 years who had not yet developed any vestibular or hearing impairment symptoms. Unfortunately there were no affected family members examined between the ages of 31 and 41; however, five persons clearly remembered the distinct appearance of vestibular symptoms prior to hearing impairment symptoms.

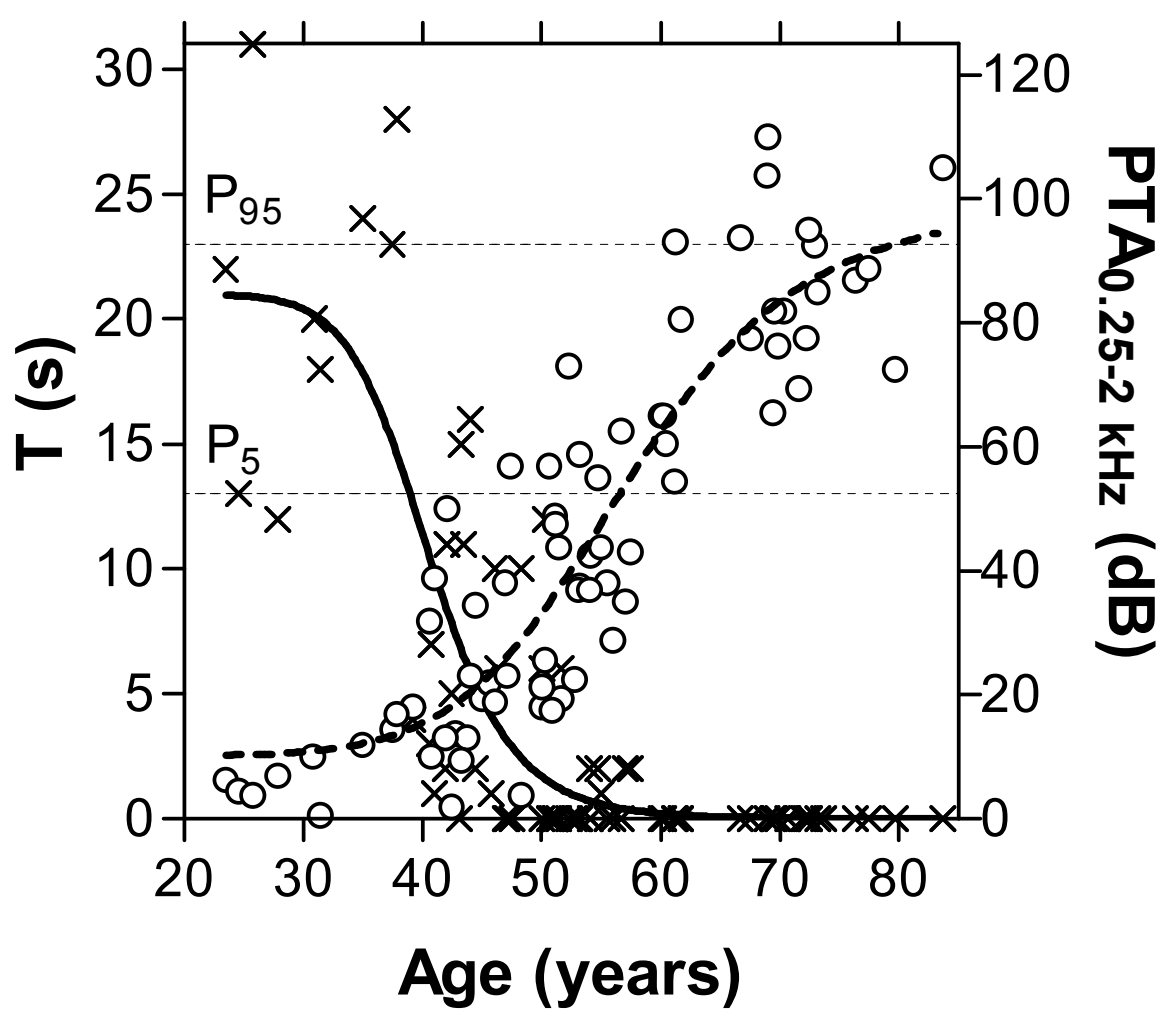

Figure 5. Mean VOR time constant (symbol $x$, solid sigmoidal curve in bold, $\mathrm{T}$ in s, left $Y$ axis) plotted against age (years) for all available $(n=74)$ P51S COCH mutation carriers including the present mutation carriers $(n=22)$. An arbitrary zero represents vestibular areflexia. The $5^{\text {th }}$ and $95^{\text {th }}$ normal percentile values are shown as dashed horizontal hairlines. For comparison, simultaneously measured PTA $0.25-2 \mathrm{kHz}$ values ( $\mathrm{dB}$ hearing level) data of all mutation carriers (open circles, dashed curve in bold, right axis) are included. See Table 3 for parameter values of sigmoidal curves.

Figure 5 shows the value of the response parameter $\mathrm{T}$ plotted against age for all carriers of the P51S mutation $(n=74)$, including the carriers of the present family $(n=22)$. In case of vestibular areflexia, arbitrary zeros were plotted. Because no significant difference was found of any fitted regression parameter value (data not shown) in the present mutation carriers $(n=22)$ compared with those of the other carriers $(n=52)$, pooling of data was performed. T decreased from a top level of 21 seconds to virtually zero. In the initial stage of vestibular dysfunction, some of the carriers showed long time constants $(\mathrm{T}>23 \mathrm{~s}$ ), characteristic of vestibular hyper- 
reactivity. The plot demonstrates that hyporeflexia $(\mathrm{T}<13 \mathrm{~s})$ is likely to appear by the end of the fourth decade of life and severe hyporeflexia $(T<5 \mathrm{~s})$ somewhere in the fifth decade.

Table 3. Results of nonlinear regression analysis (sigmoidal model, equation below) of $Y$, i.e. threshold (binaural mean PTA $0.25-2 \mathrm{kHz}$ ) or VOR time constant $T$, on $X$, i.e. age in the P51S mutation carriers.

\begin{tabular}{|c|c|c|c|}
\hline \multicolumn{4}{|c|}{ Parameter } \\
\hline $\mathrm{Y}$ & $\begin{array}{l}\text { PTA } \\
\text { (dB HL) }\end{array}$ & $\begin{array}{l}\mathrm{T} \\
(\mathrm{s})\end{array}$ & Comment \\
\hline $\mathrm{N}$ & 74 & 74 & \\
\hline Bottom & $10 \pm 5^{a}$ & 0 constant & $\begin{array}{l}\text { Offset level } \\
(0 \% \text { of deterioration trajectory) }\end{array}$ \\
\hline Top & $99 \pm 11$ & $21 \pm 2$ & $\begin{array}{l}\text { Saturation (completion) level } \\
\text { (100\% of deterioration trajectory) }\end{array}$ \\
\hline $\mathrm{H}^{*}$ & $7.7 \pm 2.1$ & $-12 \pm 3$ & $\begin{array}{l}\text { Hill slope, slope factor } \\
\text { or Hill coefficient }\end{array}$ \\
\hline$X_{10^{*}}$ & $43(38-49)^{b}$ & $49(45-53)$ & $\begin{array}{l}\text { Onset age (hearing) and } \\
\text { completion age (vestibular) of impairment }\end{array}$ \\
\hline$X_{50} *$ & $57(53-62)$ & $41(38-43)$ & $\begin{array}{l}\text { Age at } 50 \% \text { of } \\
\text { deterioration trajectory }\end{array}$ \\
\hline$X_{90}{ }^{*}$ & 76 (61-95) & $34(29-39)$ & $\begin{array}{l}\text { Onset age (vestibular) and } \\
\text { completion age (hearing) of impairment }\end{array}$ \\
\hline \multicolumn{4}{|c|}{$\begin{array}{l}\text { Equation } \\
Y=\text { Bottom }+(\text { Top-Bottom }) /\left(1+10^{(\log \times 50-\log X) \mathrm{H}}\right) \\
X \text {, age }(\text { years }) ; Y, T(\mathrm{~s}) \text { or PTA } 0.25-2 \mathrm{kHz}(\mathrm{dB} H \mathrm{HL})\end{array}$} \\
\hline
\end{tabular}

Onset age of obvious deterioration of the VOR $\left(X_{90}\right)$ was estimated at 34 years of age (Table 3). From that age onwards, T showed a maximum decrease of 1.5 seconds per year (Figure 5). Onset of vestibular impairment seems to occur earlier than onset of obvious progression in hearing impairment. We therefore tested for the pooled, simultaneously collected hearing threshold and vestibular response data in the P51S mutation carriers $(n=74)$ whether the difference in onset age was significant in a formal way, that is, pertaining to $\log \mathrm{X}_{90}$ (vestibular, regression of $\mathrm{T}$ on $\log$ age) and $\log \mathrm{X}_{10}$ (hearing loss, regression of $\mathrm{PTA}_{0.25-2 \mathrm{kHz}}$ on log age). We chose the PTA $0.25-2 \mathrm{kHz}$ to represent the threshold findings, first because the sigmoidal regression curves were fairly similar within the frequency range of 0.25 
to $2 \mathrm{kHz}$ and second because there was reason to believe that regression at 4 to 8 $\mathrm{kHz}$ was biased. ${ }^{12}$ Student's $\mathrm{t}$ test indeed revealed a significant difference. Similar findings were obtained when testing the ages at which $50 \%$ or $90 \%$ completion of progression (vestibular versus hearing impairment) had been accomplished (Table 3). Notably, the difference between the 90\% completion ages (49 and 76 years, respectively) was far greater (27 years) than the difference between the onset ages (9 years). Such a finding suggests higher progression of vestibular impairment than of hearing impairment, although the difference in Hill slope between the curves was not significant (see below). It was also remarkable that the VOR was lost, whereas residual hearing persisted (Table 3, top level, i.e. 100\% completion of progression trajectory for PTA $0.25-2 \mathrm{kHz}$, was $99 \mathrm{~dB}$ hearing level).
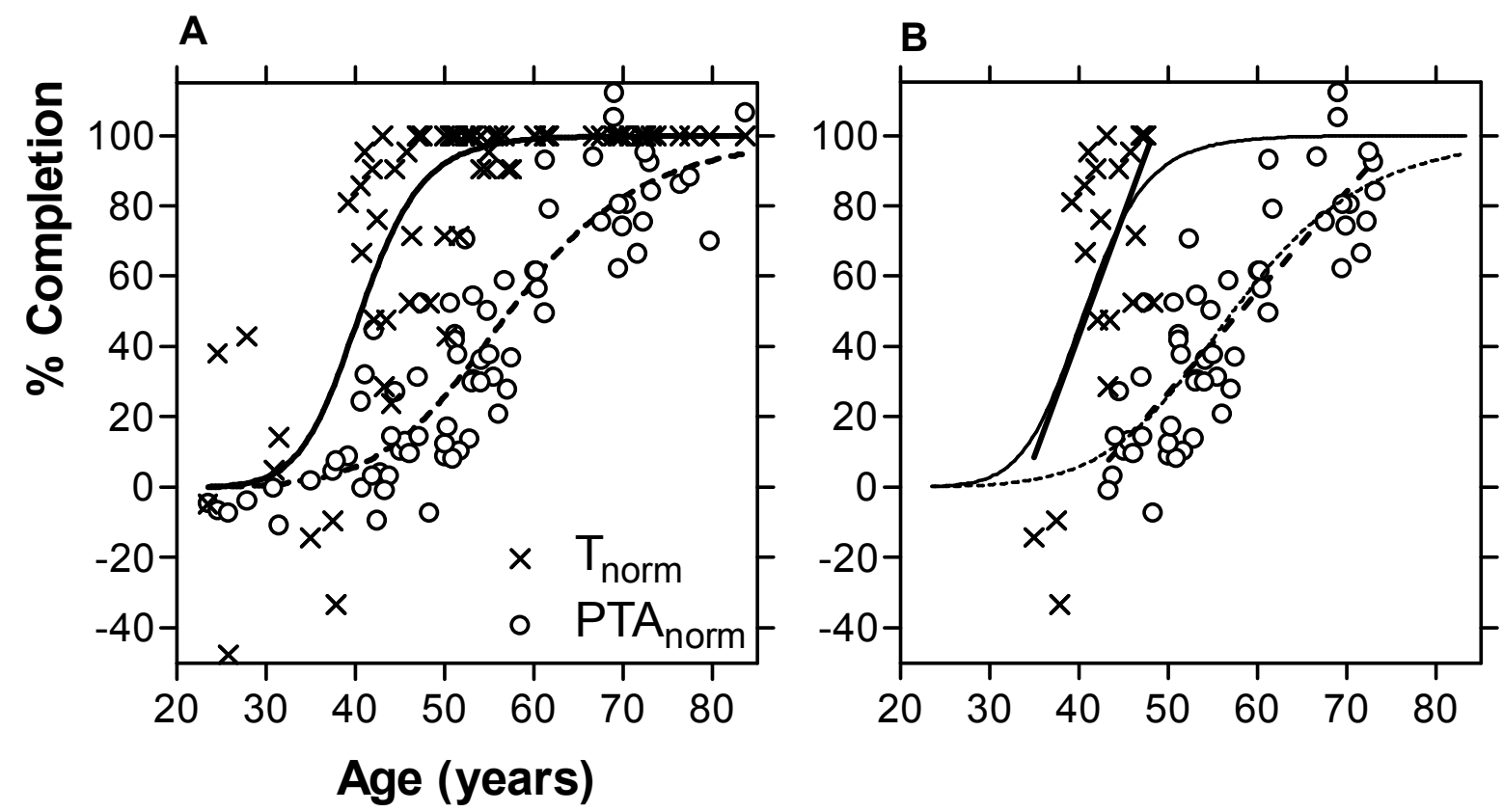

Figure 6. Normalizing transformations (0-100\% completion scale) for $\mathrm{T}$ ( $\mathrm{T}_{\mathrm{norm}}$, symbol $\mathrm{x}$ and solid line) and PTA $0.25-2 \mathrm{kHz}$ (PTAnorm, open circles and dashed line) plotted against age (A) and linear regression lines (bold, solid for T, dashed for PTA) fitted for $X_{10}<X<X_{90}$ (B). Thin curves in part B are the same as bold ones in part A. Note that $0 \%$ completion of impairment corresponds to $\mathrm{T}=21 \mathrm{~s}$ and $\mathrm{PTA}_{0.25-2 \mathrm{kHz}}=10 \mathrm{~dB}$ hearing level, respectively (see Table 3); 100\% completion of impairment corresponds to $\mathrm{T}=0$ and PTA $0.25-2 \mathrm{kHz}=99$ $\mathrm{dB}$ hearing level, respectively, and the curve spans $21 \mathrm{~s}$ for $\mathrm{T}$ and $89 \mathrm{~dB}$ hearing level for PTA $0.25-2 \mathrm{kHz}$. 
To facilitate a comparison between the trajectories outlining the deterioration of vestibular function and hearing, the data were normalized (Figure 6). The sigmoidal curves were now fitted with constant values for top (100\% completion) and bottom ( $0 \%$ completion), which might improve the fit of Hill slope. The values of Hill slope still did not differ significantly between the curves (Figure $6 \mathrm{~A})$. We therefore focused the analysis on slope and fitted the linear regression line close to the inflection point of the sigmoidal curve for $\mathrm{X}_{10}<\mathrm{X}<\mathrm{X}_{90}$ to obtain an estimate of local average slope, similar to the analyses of phoneme scores (Methods). The slope, now equivalent to a deterioration rate, was $6.9 \%$ per year for normalized $\mathrm{T}$ and $2.9 \%$ per year for normalized PTA $0.25-2 \mathrm{kHz}$, respectively (Figure 6B). ANCOVA indicated that the slopes were significantly different.

\section{Discussion}

In this report, a new, large family is described with the frequently observed P51S mutation in the $\mathrm{COCH}$ gene and cochleovestibular features similar to those previously outlined in other P51S mutation carriers. Sensorineural hearing impairment was predominant in the high frequencies and started at the lower frequencies, presumably from about 40 years onwards. Asymmetric thresholds and variable progression occur often in P51S COCH families. ${ }^{10,17,27}$ The present family showed similar types of audiograms and ATD, as has been previously described in other P51S COCH families. ${ }^{10,12,17}$ Therefore, the picture emerging from the present cross-sectional analysis of the pooled threshold-on-age data can be regarded as representative for all P51S COCH mutation carriers.

The results of the analysis of speech recognition scores in the present family, were comparable to those previously reported for P51S carriers from several different families. ${ }^{11}$ Specific retrocochlear pathology could not be traced.

The present cross-sectional data of vestibular impairment were similar to those previously reported for several different P51S COCH families. 1, 10,13,15-17 For this reason, the present analysis can also be taken as representative for all P51S

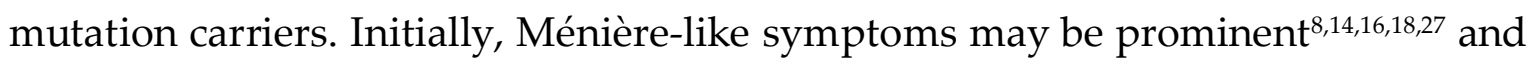
vestibular hyperreactivity may be found. ${ }^{16}$ Later on, loss of vestibular function develops, eventually leading to vestibular areflexia. Complete failure of function develops not only in the semicircular canal system but also in the otolith system. ${ }^{15}$ Head-movement-dependent oscillopsia therefore develops in all of these mutation carriers and underwater orientation becomes impossible. Enhancement of the cervico-ocular reflex attests to the absence of VOR activity. ${ }^{28}$ This enhanced reflex, 
as well as enhanced optokinetic nystagmus responses, ${ }^{29}$ compensate for at least part of the deficit of the afferent vestibular system.

The present quantitative data analysis outlines the development of progressive vestibular failure developing from the fourth decade into the sixth decade of life. It appeared that vestibular impairment developed by about 9 years earlier and deteriorated more rapid ( $4 \%$ per year) than hearing impairment. Deterioration of vestibular function had almost completed when hearing deterioration had only just begun to develop (Figures 5 and 6, Table 3). In addition, residual hearing persisted into old age.

\section{Concluding remarks}

The analyses performed in the present family provided convincing evidence that all key features were comparable across all Dutch P51S COCH mutation carriers examined, including the present ones. The development of vestibular failure could now be analysed in a formal, quantitative way, not only for the present subjects but also for the whole ensemble of mutation carriers. A similar analysis of the development of hearing impairment with advancing age, based on simultaneous original measurements in the same mutation carriers, some still being asymptomatic, allowed for statistical testing between the respective onset ages and slopes. It showed that vestibular deterioration starts earlier, progresses more rapidly and, eventually, is more complete than hearing deterioration in P51S $\mathrm{COCH}$ mutation carriers.

\section{References}

1. Verhagen WIM, Huygen PLM, Joosten EMG. Familial progressive vestibulocochlear dysfunction. Arch Neurol 1988;45:766-768.

2. Manolis EN, Yandavi N, Nadol Jr JB, Eavy RD, McKenna M, Rosenbaum S, Khetarpal U, Halpin C, Merchant SN, Duyk GM, MacRae C, Seidman CE, Seidman JG. A gene for nonsyndromic autosomal dominant progressive postlingual sensorineural hearing loss maps to chromosome 14q12-13. Hum Mol Genet 1996;5:1047-1050.

3. Robertson NG, Skvorak AB, Yin Y, Weremowicz S, Johnson KR, Kovatch KA, Battey JF, Bieber FR, Morton CC. Mapping and characterization of a novel cochlear gene in human and in mouse: a positional candidate gene for a deafness disorder, DFNA9. Genomics 1997; 46:345-354.

4. Kamarinos M, McGill J, Lynch M, Dahl H. Identification of a novel COCH mutation, I109N, highlights the similar clinical features observed in DFNA9 families. Hum Mutat 2001;17:351. Erratum in: Hum Mutat 2001;18:547-548.

5. de Kok YJM, Bom SJH, Brunt TM, Kemperman MH, van Beusekom E, van der Velde-Visser SD, Robertson NG, Morton CC, Huygen PLM, Verhagen WIM, Brunner HG, Cremers CWRJ, Cremers FPM. A Pro51Ser mutation in the $\mathrm{COCH}$ gene is associated with late onset autosomal dominant progressive sensorineural hearing loss with vestibular defects. Hum Mol Genet 1999;8:361-366. 
6. Robertson NG, Lu L, Heller S, Eavey RD, McKenna M, Nadol JB Jr, Miyamoto RT, Linthicum FH Jr, Lubianca Neto JF, Hudspeth AJ, Seidman CE, Morton CC, Seidman JG. Mutations in a novel cochlear gene cause DFNA9, a human nonsyndromic deafness with vestibular dysfunction. Nat Genet 1998;20:299-303.

7. Khetarpal U, Schuknecht HF, Gacek RR, Holmes LB. Autosomal dominant sensorineural hearing loss. Pedigrees, audiologic findings, and temporal bone findings in two kindreds. Arch Otolaryngol Head Neck Surg 1991;117:1032-1042.

8. Fransen E, Verstreken M, Verhagen WIM, Wuyts FL, Huygen PLM, D'Haese P, Robertson NG, Morton CC, McGuirt WT, Smith RJH, Declau F, Van de Heyning PH, Van Camp G. High prevalence of symptoms of Menière's disease in three families with a mutation in the COCH gene. Hum Mol Genet 1999;8:1425-1429.

9. Tamagawa $Y$, Ishikawa K, Ishikawa K, Ishida T, Kitamura K, Makino S, Tsuru T, Ichimura K. Phenotype of DFNA11: A nonsyndromic hearing loss caused by a Myosin VIIA mutation. Laryngoscope 2002;112:292-297.

10. Bom SJH, Kemperman MH, De Kok YJM, Huygen PLM, Verhagen WIM, Cremers FPM, Cremers CWRJ. Progressive cochleovestibular impairment caused by a point mutation in the COCH gene at DFNA9. Laryngoscope 1999;109:1525-1530.

11. Bom SJH, De Leenheer EMR, Lemaire FX, Kemperman MH, Verhagen WIM, Marres HAM, Kunst HPM, Ensink RJH, Bosman AJ, Van Camp G, Cremers FPM, Huygen PLM, Cremers CWRJ. Speech recognition scores related to age and degree of hearing impairment in DFNA2/KCNQ4 and DFNA9/COCH. Arch Otolaryngol Head Neck Surg 2001;127:10451048.

12. Bom SJH, Kemperman MH, Huygen PLM, Luijendijk MWJ, Cremers CWRJ. Cross-sectional analysis of hearing threshold in relation to age in a large family with cochleovestibular impairment thoroughly genotyped for DFNA9/COCH. Ann Otol Rhinol Laryngol 2003; 112:280-286.

13. Verhagen WIM, Huygen PLM, Theunissen EJJM, Joosten EMG. Hereditary vestibulocochlear dysfunction and vascular disorders. J Neurol Sci 1989;92:55-63.

14. Verhagen WIM, Huygen PLM. Familial progressive vestibulocochlear dysfunction (letter). Arch Neurol 1991;48:262.

15. Verhagen WIM, Huygen PLM, Bles W. A new autosomal dominant syndrome of idiopathic progressive vestibulo-cochlear dysfunction with middle-age onset. Acta Otolaryngol 1992; 112:899-906.

16. Verhagen WIM, Bom SJH, Huygen PLM, Fransen E, Van Camp G, Cremers CWRJ. Familial progressive vestibulocochlear dysfunction caused by a $\mathrm{COCH}$ mutation (DFNA9). Arch Neurol 2000;57:1045-1047.

17. Verhagen WIM, Bom SJH, Fransen E, Van Camp G, Huygen PLM, Theunissen EJJM, Cremers CWRJ. Hereditary cochleovestibular dysfunction due to a $\mathrm{COCH}$ gene mutation (DFNA9): a follow-up study of a family. Clin Otolaryngol 2001;26:477-483.

18. Lemaire FX, Feenstra L, Huygen PLM, Fransen E, Devriendt K, Van Camp G, Vantrappen G, Cremers CWRJ. Progressive late-onset sensorineural hearing loss and vestibular impairment with vertigo (DFNA9/COCH). Longitudinal analyses in a belgian family. Otol Neurotol 2003;24:743-748.

19. Bischoff AMLC, Pauw RJ, Huygen PLM, Aandekerk AL, Kremer H, Cremers CWRJ, Cruysberg JRM. Vertical Corneal Striae in Families with Autosomal Dominant Hearing Loss: DFNA9/COCH. Am J Ophthalmol 2007. In press.

20. ISO 389. Acoustics. Standard reference zero for the calibration of pure tone air conduction audiometers. Geneva: International Organisation for Standardization, 1985.

21. ISO 8253-1.Acoustics. Audiometric test methods, I: basic pure tone air and bone conduction threshold audiometry. Geneva: International Organisation for Standardization, 1989. 
22. ISO 7029. Acoustics. Threshold as hearing by air conduction as a function of age and sex for otologically normal persons. Geneva: International Organisation for Standardization, 1984.

23. Marres HAM, van Ewijk M, Huygen PLM, Kunst HPM, van Camp G, Coucke PJ, Willems PJ, Cremers CWRJ. Inherited nonsyndromic hearing loss. An audiovestibular study in a large family with autosomal dominant progressive hearing loss related to DFNA2. Arch Otolaryngol Head Neck Surg 1997;123:573-577.

24. Theunissen EJJM, Huygen PLM, Folgering HT. Vestibular hyperreactivity and hyperventilation. Clin Otolaryngol 1986;11:161-169.

25. Fernández C, Goldberg JM. Physiology of peripheral neurons innervating semicircular canals of the squirrel monkey. I. Response to sinusoidal stimulations and dynamics of peripheral vestibular system. J Neurophysiol 1971;34:661-674.

26. Bles W, de Jong JMBV, de Wit G. Somatosensory compensation for loss of labyrinthine function. Acta Otolaryngol 1984;97:213-221.

27. Verstreken M, Declau F, Wuyts FL, D'Haese P, Van Camp G, Fransen E, Van den Hauwe L, Buyle S, Smets REM, Feenstra L, Van der Stappen A, Van de Heyning PH. Hereditary otovestibular dysfunction and Ménière's disease in a large Belgian family is caused by a missense mutation in the COCH gene. Otol Neurotol 2001;22:874-881.

28. Huygen PLM, Verhagen WIM, Nicolasen MGM. Cervico-ocular reflex enhancement in labyrinthine-defective and normal subjects. Exp Brain Res 1991;87:457-464.

29. Huygen PLM, Verhagen WIM, Theunissen EJJM, Nicolasen MGM. Compensation of total loss of vestibulo-ocular reflex by enhanced optokinetic response. Acta Otolaryngol 1989; 468:359-364. 


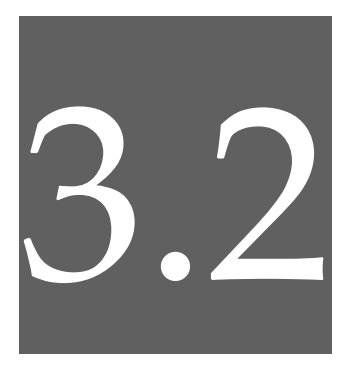

\section{Vertical corneal striae in families with autosomal dominant hearing loss: DFNA9/COCH}

A.M.L.C. Bischoff

R.J. Pauw

P.L.M. Huygen

A.L. Aandekerk

H. Kremer

C.W.R.J. Cremers

J.R.M. Cruysberg

American Journal Ophthalmology 2007, in press. 



\section{Abstract}

Purpose: Investigation of a possible association between vertical corneal striae and mutations in the $\mathrm{COCH}$ gene, observed in four DFNA9 families with autosomal dominant hearing loss and vestibular dysfunction.

Design: Prospective case-series.

Methods: Ophthalmologic examinations with photography of the cornea after instillation of fluorescein were performed in 98 family members with 61 mutation carriers of four DFNA9 families at the Radboud University Nijmegen Medical Centre. Families 1 and 2 harbour the Pro51Ser mutation, and families 3 and 4 harbour the Gly88Glu and the Gly87Trp mutation, respectively. Statistical analysis was performed to find an association between the vertical corneal striae and the $\mathrm{COCH}$ mutation for each family and to test whether the four families were different in this respect.

Results: The vertical corneal striae were exclusively visible after instillation of fluorescein. They caused minor problems, as dry eye symptoms, and were not present in the general Dutch ophthalmologic population. The striae were present from an age of 47 years in 32 individuals, of whom 27 individuals had a $\mathrm{COCH}$ mutation. Statistical analysis on the striae and the $\mathrm{COCH}$ mutations showed a significant association in families 1,2 and 3 ( $\mathrm{p}=0.0006$ ), but not in family 4 $(\mathrm{p}=0.63)$.

Conclusions: Data analysis demonstrated a significant association between vertical corneal striae and the Pro51Ser and Gly88Glu mutations in the $\mathrm{COCH}$ gene in DFNA9 families 1, 2 and 3 with cochleovestibular dysfunction. Our findings suggest that the vertical corneal striae and cochleovestibular dysfunction may be caused by the same $\mathrm{COCH}$ mutations.

\section{Introduction}

Non-syndromic autosomal dominantly inherited forms of hearing loss are designated as "DFNA", followed by the number of identification in chronological sequence. DFNA9/COCH causes mid-life onset of progressive hearing loss, predominantly in the high frequencies, with mid-life onset of progressive vestibular dysfunction. ${ }^{1-15}$ At present, nine different exonic and non-synonymous mutations have been identified in the $\mathrm{COCH}$ gene: Val66Gly, ${ }^{16}$ Gly88Glu, ${ }^{16}$ Trp117Arg, ${ }^{16}$ Pro51Ser, ${ }^{7,17}$ Ile109Asn, ${ }^{10}$ Ala119Thr, ${ }^{18}$ Val104del ${ }^{19}{ }^{1}$ Cys542Phe ${ }^{20}$ and Gly87Trp..$^{21}$ All mutations affect the LCCL domain of the protein, ${ }^{22}$ except for the mutation causing the Cys542Phe substitution, which is located at the vWFA2 domain. ${ }^{20}$ The function of cochlin is still unclear. $\mathrm{COCH}$ encodes the extracellular 
matrix protein cochlin, which is expressed in the cochlear fibrocytes of the spiral ligament, spiral limbus and osseous spiral lamina and in the fibrocytes of the stroma underlying the sensory epithelium of the crista ampullaris of the semicircular canals..$^{23,24}$

We discovered a peculiar pattern of mid-life onset vertical corneal striae in three families with a mutation in the $\mathrm{COCH}$ gene, which is not found in the general Dutch ophthalmologic population. This is the first report on structural abnormalities in the anterior part of the eye in DFNA9/COCH. Ocular abnormalities in non-syndromic autosomal dominant sensorineural hearing loss have only been reported earlier in DFNA11/MYO7A with subclinical retinal involvement. ${ }^{25}$

\section{Methods}

Ninety-eight family members of four Dutch DFNA9/COCH families participated in this study at the Radboud University Nijmegen Medical Centre. Families 1 and 2 carried the Pro51Ser mutation, ${ }^{8,15}$ family 3 carried the Gly88Glu mutation, ${ }^{13}$ and the Gly87Trp mutation was present in family $4 . .^{21}$ The mutations were identified by restriction digestion in the first two families ${ }^{8,15}$ and by sequencing the gene in the third and fourth family. ${ }^{13,21}$ The study was approved by the local medical ethics committee. Signed informed consent was obtained from all participants. Medical history was recorded with special attention to ocular symptoms. Ophthalmologic history data were retrieved. Haplotype analysis proved that families 1 and 2 have the same common founder as has been previously shown for the majority of the investigated families with the Pro51Ser mutation. ${ }^{26}$

Slit-lamp examination was performed with and without fluorescein paper strips, using the slit-lamp microscope with a broad beam and a cobalt blue filter. Corneas were photographed by tangential illumination of the limbus with white light and after application of fluorescein. After fluorescein installation in the lower fornix, the patient was asked to blink several times. Rubbing the eyes was strictly avoided. The corneal surface curvature was measured by Javal keratometry and the corneal thickness was tested by ultrasound pachymetry. Best corrected visual acuity was documented. Intraocular pressure was determined by Goldmann applanation tonometry. The Schirmer test was used to evaluate tear production. To screen for possible age-dependent differences, intraocular pressure, keratometry, Schirmer test, and visual acuity data were plotted against age. Both mutation carriers with the vertical striae and carriers who did not exhibit these striae were compared. 


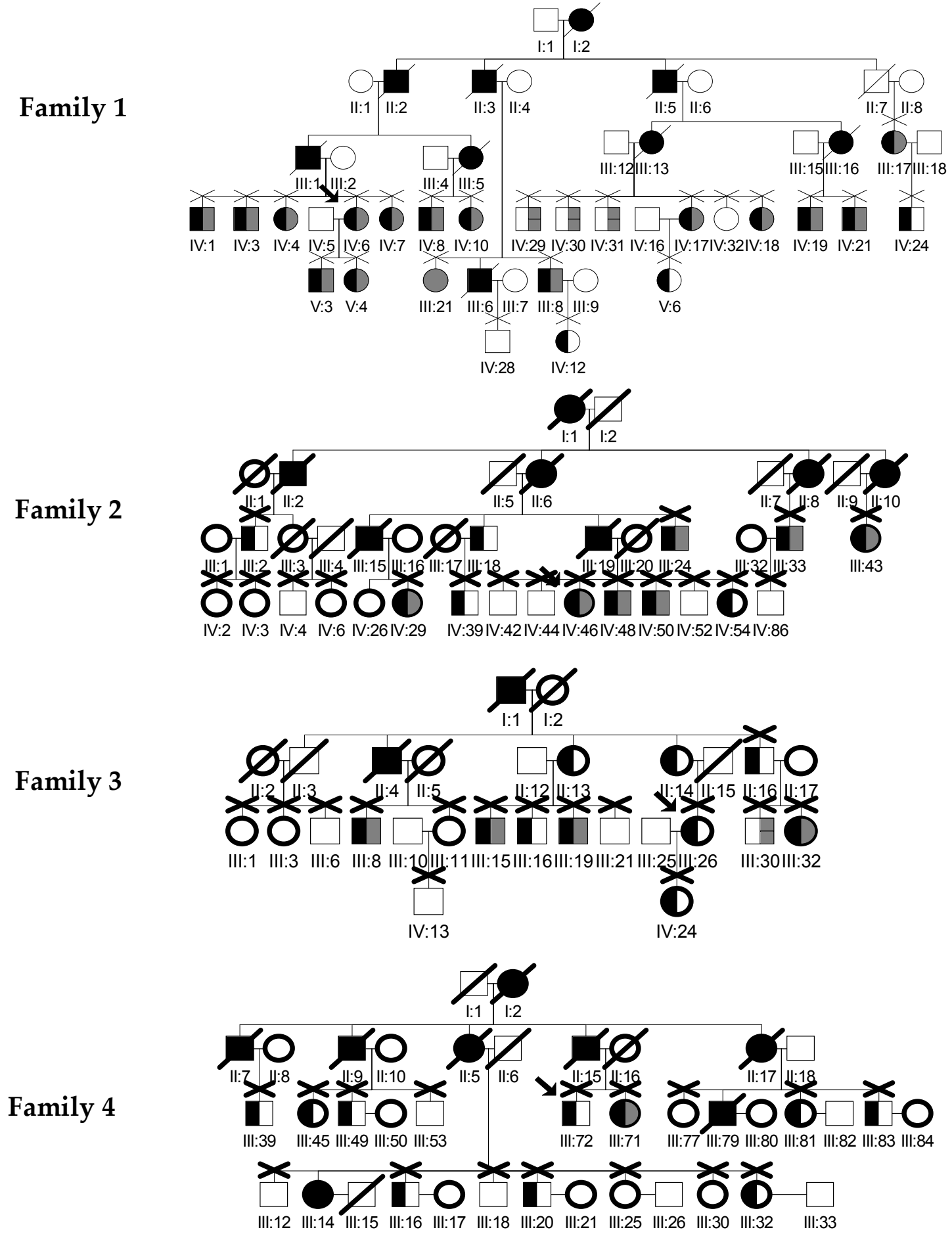

Figure 1. Pedigrees of DFNA9 families 1 to 4 with vertical corneal striae. A cross above the symbol indicates that ophthalmologic examination has been performed. Grey right half of the symbol = vertical corneal striae; black left half of the symbol = $\mathrm{COCH}$ mutation; open right half symbol $=$ no vertical corneal striae; open left half symbol $=$ no $C O C H$ mutation; square $=$ man; circle $=$ woman; Slashed symbol $=$ deceased person; completely black symbol = clinically affected individual, not genetically tested; arrow = proband. The individuals have the same identification number as in the original pedigrees. The pedigrees have been simplified. The youngest generation is not shown for privacy reasons. Please note that generation lines of different lengths have been used in order to compact the figure. 
Fisher's exact test and chi-square test were performed on separate and grouped family data to detect a possible association between the occurrences of the vertical corneal striae and the $\mathrm{COCH}$ mutation.

\section{Results}

In the pedigrees of the four DFNA9/COCH families shown in Figure 1, the presence of both the vertical corneal striae and the $\mathrm{COCH}$ mutation are indicated. The first number of the identification code is the number of the family. The second number (after the dot) represents the identification code of the patient in the original pedigree. The mean age of the participants was 50.6 years. Sixty-one individuals were mutation carriers. Vertical corneal striae were present in 32 individuals, of whom 27 had a $\mathrm{COCH}$ mutation. Surprisingly, the striae were also found in corneas of four members of family 1 without a $\mathrm{COCH}$ mutation (1.IV29$31,1 . I I I 21)$ and in one member of family 3 without a COCH mutation (3.III30). In family 1 , three were siblings and one of them only showed vertical corneal striae exclusively on the right cornea. In family 4, only one of the 19 mutation carriers (4.III71) had vertical corneal striae in the right eye. This person had keratoconus of both eyes and the left cornea had been transplanted.

Most of the affected persons had dry eye symptoms and showed conjunctival hyperaemia and instability of refraction and vision. Foreign body sensation and photophobia were also prominent symptoms. Several persons experienced problematic adjustment to glasses because of refraction instability. The proband of family 1 (1.IV6) had the most severe symptoms. Because of severely dry eyes, she had permanent lacrimal punctal plugs inserted bilaterally. She and her sister (1.IV4) complained about such fluctuations in vision for years to the extent that spectacles no longer helped. They had used bandage lenses for years. Only two persons $(1 . \mathrm{V} 3,1 . \mathrm{V} 13)$ used hard contact lenses, of which individual 1.V3 showed the vertical corneal striae.

Slit-lamp examination with fluorescein revealed spontaneously visible (i.e. not elicited by rubbing) vertical corneal striae highlighted in the superficial layers of the central cornea (Figure 2). However, in one person without a $\mathrm{COCH}$ mutation (3.III30), the striae were only visible in the upper half of both corneas. Illuminated striae were visible against a dark blue background. The vertical corneal striae were best visible after cessation of blinking for about ten seconds and gradually vanished after a few minutes. The vertical corneal striae had a mid-life onset, as they were visible from an age of about 47 years onwards. In the younger generation, only the two siblings of the proband (1.V3, 4; age 25, 23 years) showed very faint striae. The pattern of the striae appeared to be reproducible: photo- 
graphs taken one hour to several months later still showed the same pattern of striae (Figure 2). As a rule, rubbing was avoided, but, apart from that, rubbing was done deliberately in some cases to see if this changed the pattern. The vertical corneal striae became more pronounced by rubbing, but the pattern itself did not change. Efforts to expose the vertical corneal striae by tangential illumination of the limbus with white light were unsuccessful. Ultrasound pachymetry of the central cornea of eight eyes (four patients) with vertical corneal striae were within normal limits (mean, $519 \mu \mathrm{m}$; range 507-554 $\mu \mathrm{m}$ ). Other results of ophthalmologic examinations are shown in the appendix table.

A

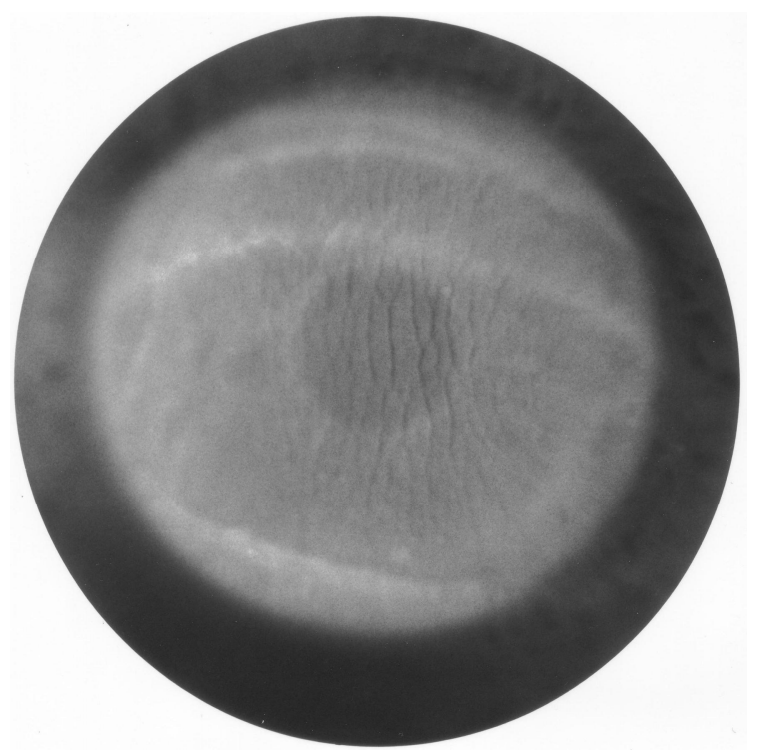

B

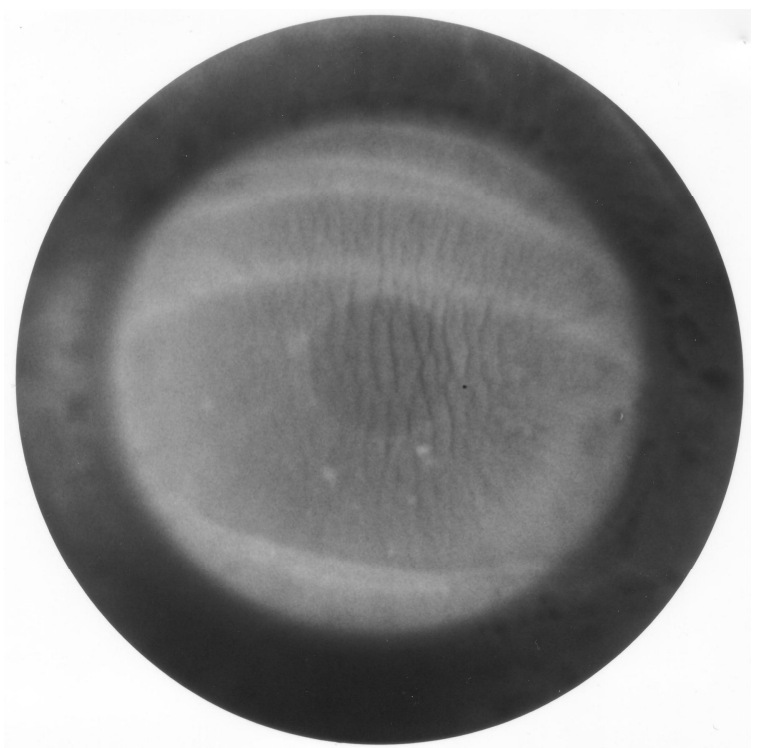

Figure 2. Vertical corneal striae in one person with DFNA9. Corneal photographs of case 1.IV6 (2A), and of case 1.IV6 taken two weeks later (2B), showing the typical vertical corneal striae to be reproducible.

The results of intraocular pressure measurements, Schirmer test, Javal keratometry, and visual acuity test of the mutation carriers with versus without the vertical corneal striae were compared (Figure 3), to find out whether there were age-dependent differences. The mutation carriers with the vertical corneal striae tended to show higher intraocular pressure and a lower corneal radius. However, no significant difference in either of these parameters could be detected between mutation carriers with or without striae (Figure 3A and 3C). Intraocular pressures were within the normal range. Most of the mutation carriers with the striae had reduced tear production with the Schirmer test (Figure 3B), in line with their symptoms and clinical examination. There was no significant difference detected in visual acuity. 
A Intraocular pressure

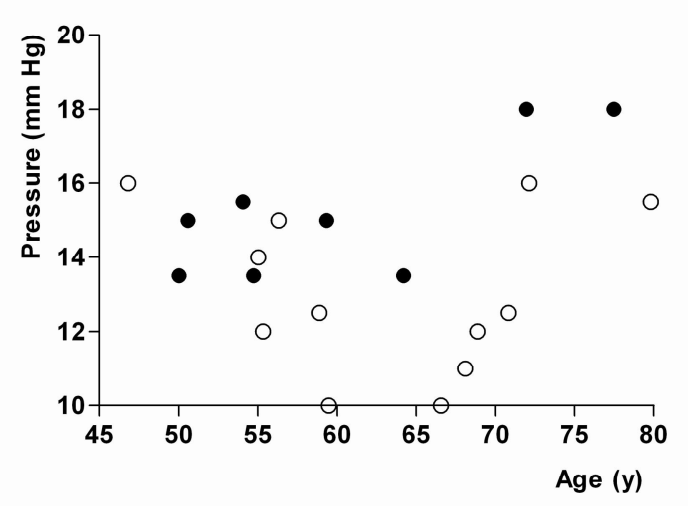

C Javal keratometry

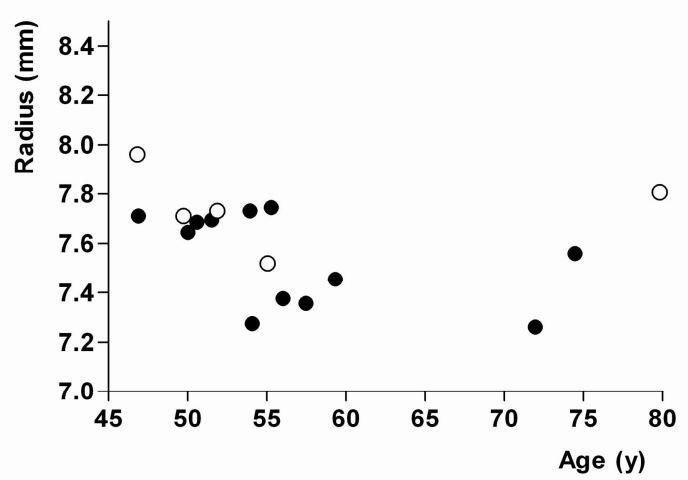

B
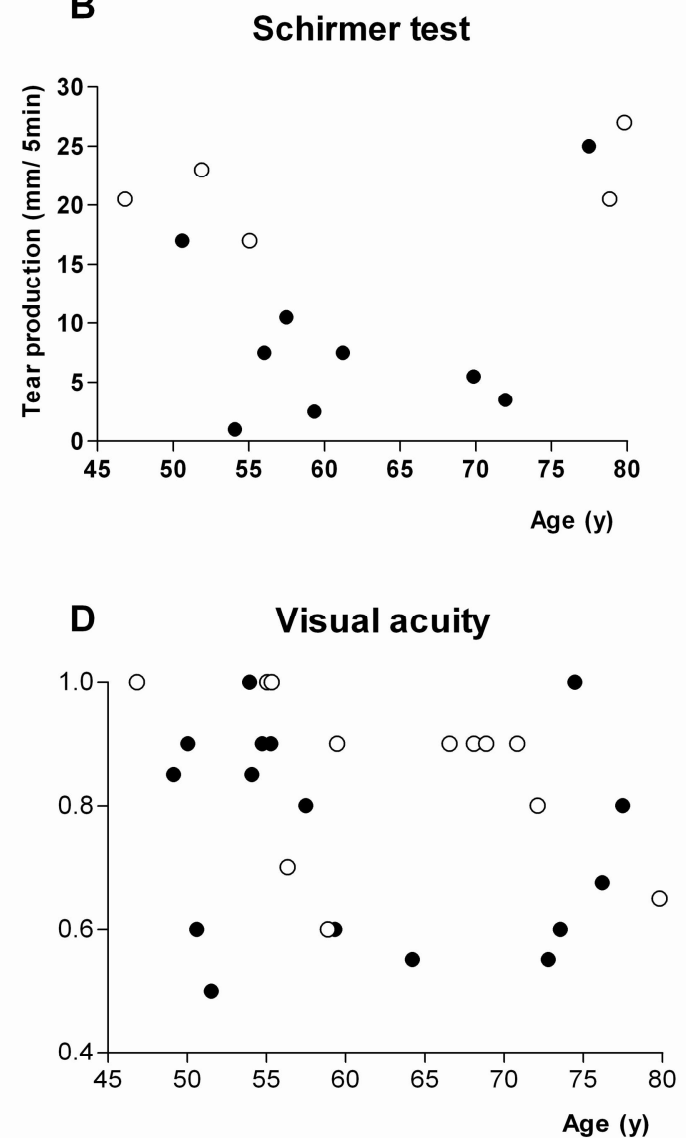

Figure 3. Ocular testing of DFNA9 family members with and without the vertical corneal striae. Comparison of the ocular test results between mutation carriers with the vertical corneal striae (filled circles) and mutation carriers without the striae (open circles) plotted against age. Figure 3A. Results of intraocular pressure in mmHg. Figure 3B. Results of Schirmer lacrimation test in $\mathrm{mm}$ per $5 \mathrm{~min}$. Figure 3C. Results of Javal keratometry readings in $\mathrm{mm}$. Figure 3D. Results of visual acuity test.

The percentage prevalence of vertical corneal striae in mutation carriers in families 1 to 4 (age > 47 years) was 93, 78, 57, and 10 (\%) respectively ( $\mathrm{n}_{\text {total, fam1- }}$ ${ }_{4}=40$ ). With Fisher's exact test a significant association was found between the presence of the $\mathrm{COCH}$ mutation and the vertical corneal striae in families 1, 2, and $3(p=0.0006)$, but not in family $4(p=0.63)$. There was no significant difference in prevalence detected among the first three families $(\mathrm{p}=0.37)$.

\section{Discussion}

The spontaneously visible vertical corneal striae were present in 32 individuals of four DFNA9/COCH families, of whom 27 were mutation carriers. A significant association between the cochleovestibular and corneal features was found in families 1, 2, and 3, but not in family 4 . Because families 1, 2, and 3 harbour two 
different $\mathrm{COCH}$ mutations (Pro51Ser and Gly88Glu) and the vertical corneal striae are not found in the normal Dutch ophthalmologic population, the association of the $\mathrm{COCH}$ mutations and the corneal vertical striae suggests that mutations in the $\mathrm{COCH}$ gene can cause both the cochleovestibular and the corneal features. This is corroborated by the fact that transcripts of the $\mathrm{COCH}$ gene can be detected in the cornea by RT-PCR (Kremer, unpublished data). There is as yet no clear explanation why the corneal phenotype was not present in family 4 (Gly87Trp), and why the vertical corneal striae also were present in five individuals without a proven $\mathrm{COCH}$ mutation.

The possibility of two different neighbour genes causing cosegregation of two phenotypes could still not be ruled out. Recombination events could explain why four persons in family 1 and one person in family 2 with vertical corneal striae did not have the mutation in the $\mathrm{COCH}$ gene and why not all $\mathrm{COCH}$ mutation carriers exhibit the striae. On the contrary, the latter can be caused by reduced penetrance. Nevertheless, this two-gene theory is not likely, because the striae are not described previously and are visible in individuals with two different mutations in the $\mathrm{COCH}$ gene.

Further support of a $\mathrm{COCH}$ gene mutation as a cause of the vertical corneal striae is the identification of co-deposits of cochlin and acidophilic mucopolysaccharide in the trabecular meshwork around Schlemm's canal of patients with primary open angle glaucoma, and in glaucomatous DBA/2J mice with progressive hearing loss. ${ }^{27,28}$ Similar abnormal acidophilic mucopolysaccharide deposits have been described in the inner ear of DFNA9 patients. ${ }^{24,30-32}$ Electron microscopic examination of the cochlea showed that the deposits consist of highly branched microfibrils that interfere with normal fibrillogenesis, causing degradation of collagen type II. ${ }^{33}$ This decrease in collagen type II was also found in the trabecular meshwork from an age of 40 to 45 years onwards. Remarkably, the cochleovestibular dysfunction becomes prominent from this age in DFNA9 patients, as well as the vertical corneal striae.

The cochleovestibular phenotype is similar in all four DFNA9/COCH families. Hearing impairment starts in the fifth decade with progression to profound hearing loss. Vestibular dysfunction begins, prior to the hearing impairment, at 35 years of age and leads to complete vestibular areflexia in families with the Pro51Ser mutation (families 1 and 2). The onset age seems to be higher in the families with the Gly88Glu and the Gly87Trp mutation (family 3 and 4). Vestibular dysfunction is fully penetrant in families with the Pro51ser mutation, and is less in the families with the Gly87Trp substitution and the Gly88Glu mutation. $8,13,15,29$ 
The vertical corneal striae in the present study were different from the anterior corneal mosaic, described by Bron. ${ }^{34-38}$ Whereas the vertical corneal striae in the DFNA9 patients were visible without any manipulation or abnormal intraocular pressure, the anterior corneal mosaic was only detectable in hypotonic eyes or in normal eyes after applying external pressure. ${ }^{35,39}$ Also, the vertical corneal striae in the present corneas had an exclusively vertical direction, but the pattern of the anterior corneal mosaic was honeycomb-like, elongated obliquely, vertically or horizontally. ${ }^{36}$

Comparison of the ophthalmologic examinations in the mutation carriers with and without the vertical corneal striae is important to investigate the pathogenesis of the vertical corneal striae. From the age of 47 years onwards, intraocular pressures in the mutation carriers with the vertical corneal striae were within normal limits, but tended to be higher than in the carriers without the striae. The results of the Schirmer test showed subnormal values in the older age group with the vertical corneal striae, supporting the association of the striae with dry eye syndrome.

These findings indicate that ophthalmologic examination of DFNA9/COCH families, especially those having a mutation different from the Pro51Ser mutation, may be worthwhile to strengthen or weaken the suggestion that $\mathrm{COCH}$ mutations cause both the cochleovestibular and corneal features. Additional studies at the molecular level of the inner ear and cornea are necessary to elucidate the pathogenic mechanism.

\section{References}

1. Verhagen WIM, Huygen PLM, Joosten EMG. Familial progressive vestibulocochlear dysfunction. Arch Neurol 1988;45:766-768.

2. Verhagen WIM, Huygen PLM, Theunissen EJJM, Joosten EMG. Hereditary vestibulocochlear dysfunction and vascular disorders. J Neurol Sci 1989;92:55-63.

3. Verhagen WIM, Huygen PLM. Familial progressive vestibulocochlear dysfunction. Arch Neurol 1991;48:262.

4. Verhagen WIM, Huygen PLM, Bles W. A new autosomal dominant syndrome of idiopathic progressive vestibulo-cochlear dysfunction with middle-age onset. Acta Otolaryngol 1992; 112:899-906.

5. Verhagen WIM, Bom SJH, Huygen PLM, Fransen E, Van Camp G, Cremers CWRJ. Familial progressive vestibulocochlear dysfunction caused by a $\mathrm{COCH}$ mutation (DFNA9). Arch Neurol 2000;57:1045-1047.

6. Verhagen WIM, Bom SJH, Fransen E, Van Camp G, Huygen PLM, Theunissen EJJM, Cremers CWRJ. Hereditary cochleovestibular dysfunction due to a $\mathrm{COCH}$ gene mutation (DFNA9): a follow-up study of a family. Clin Otolaryngol 2001;26:477-483.

7. De Kok YJM, Bom SJH, Brunt TM, Kemperman MH, van Beusekom E, van der Velde-Visser SD, Robertson NG, Morton CC, Huygen PLM, Verhagen WIM, Brunner HG, Cremers CWRJ, Cremers FPM. A Pro51Ser mutation in the $\mathrm{COCH}$ gene is associated with late onset 
autosomal dominant progressive sensorineural hearing loss with vestibular defects. Hum Mol Genet 1999;8:361-366.

8. Bom SJH, Kemperman MH, De Kok YJM, Huygen PLM, Verhagen WIM, Cremers FPM, Cremers CWRJ. Progressive cochleovestibular impairment caused by a point mutation in the COCH gene at DFNA9. Laryngoscope 1999;109:1525-1530.

9. Bom SJH, Kemperman MH, Huygen PLM, Luijendijk MWJ, Cremers CWRJ. Cross-sectional analysis of hearing threshold in relation to age in a large family with cochleovestibular impairment thoroughly genotyped for DFNA9/COCH. Ann Otol Rhinol Laryngol 2003;112: 280-286.

10. Kamarinos M, McGill J, Lynch M, Dahl H. Identification of a novel COCH mutation, I109N, highlights the similar clinical features observed in DFNA9 families. Hum Mutat 2001;17:351. Erratum in: Hum Mutat 2001;18:547-548.

11. Verstreken M, Declau F, Wuyts FL, D'Haese P, Van Camp G, Fransen E, Van den Hauwe L, Buyle S, Smets REM, Feenstra L, Van der Stappen A, Van de Heyning PH. Hereditary otovestibular dysfunction and Ménière's disease in a large Belgian family is caused by a missense mutation in the COCH gene. Otol Neurotol 2001;22:874-881.

12. Kemperman MH, Bom SJH, Lemaire FX, Verhagen WIM, Huygen PLM, Cremers CWRJ. DFNA9/COCH and its phenotype. In: Cremers CWRJ, Smith RJH (eds). Genetic Hearing Impairment. Its clinical presentations. Advances in Oto-Rhino-Laryngology, vol. 61. Basel: Karger, 2002;61:66-72.

13. Kemperman MH, De Leenheer EMR, Huygen PLM, van Duijnhoven G, Morton CC, Robertson NG, Cremers FPM, Kremer H, Cremers CWRJ. Audiometric, vestibular and genetic aspects of a DFNA9 family with a G88E COCH mutation. Otol Neurotol 2005;26:926933.

14. Lemaire FX, Feenstra L, Huygen PLM, Fransen E, Devriendt K, Van Camp G, Vantrappen G, Cremers CWRJ. Progressive late-onset sensorineural hearing loss and vestibular impairment with vertigo (DFNA9/COCH). Longitudinal analyses in a Belgian family. Otol Neurotol 2003;24:743-748.

15. Bischoff AMLC, Huygen PLM, Kemperman MH, Pennings RJE, Bom SJH, Verhagen WIM, Admiraal RJC, Kremer $\mathrm{H}$, Cremers CWRJ. Vestibular deterioration precedes hearing deterioration in the P51S COCH mutation (DFNA9). An analysis in 74 mutation carriers. Otol Neurotol 2005;26:918-925.

16. Robertson NG, Lu L, Heller S, Eavey RD, McKenna M, Nadol JB Jr, Miyamoto RT, Linthicum FH Jr, Lubianca Neto JF, Hudspeth AJ, Seidman CE, Morton CC, Seidman JG. Mutations in a novel cochlear gene cause DFNA9, a human nonsyndromic deafness with vestibular dysfunction. Nat Genet 1998;20:299-303.

17. Fransen E, Verstreken M, Verhagen WIM, Wuyts FL, Huygen PLM, D'Haese P, Robertson NG, Morton CC, McGuirt WT, Smith RJH, Declau F, Van de Heyning PH, Van Camp G. High prevalence of symptoms of Menière's disease in three families with a mutation in the COCH gene. Hum Mol Genet 1999;8:1425-1429.

18. Usami S, Takahashi K, Yuge I, Ohtsuka A, Namba A, Abe S, Fransen E, Patthy L, Otting G, Van Camp G. Mutations in the $\mathrm{COCH}$ gene are a frequent cause of autosomal dominant progressive cochleo-vestibular dysfunction, but not of Meniere's disease. Eur J Hum Genet 2003;11:744-748.

19. Nagy I, Horvath M, Trexler M, Répássy G, Patthy L. A novel COCH mutation, V104del, impairs folding of the LCCL domain of cochlin and causes progressive hearing loss. J Med Genet 2004;41:e9 (http://www.jmedgenet.com/cgi/content/ful/41/1/e9).

20. Street VA, Kallman JC, Robertson NG, Kuo SF, Morton CC, Phillips JO. A novel DFNA9 mutation in the vWFA2 domain of $\mathrm{COCH}$ alters a conserved cysteine residue and intrachain disulfide bond formation resulting in progressive hearing loss and site-specific vestibular and central oculomotor dysfunction. Am J Med Genet A 2005;139:86-95. 
21. Collin RW, Pauw RJ, Schoots J, Huygen PL, Hoefsloot LH, Cremers CW, Kremer H. Identification of a novel $\mathrm{COCH}$ mutation, G87W, causing autosomal dominant hearing disorder DFNA9. Am J Med Genet A. 2006;140A:1791-1794.

22. Liepinsh E, Trexler M, Kaikkonen A, Weigelt J, Banyai L, Patthy L, Otting G. NMR structure of the LCCL domain and implications for DFNA9 deafness disorder. EMBO J 2001;20:53475353.

23. Robertson NG, Resendes BL, Lin JS, Lee C, Aster JC, Adams JC, Morton CC. Inner ear localization of mRNA and protein products of $\mathrm{COCH}$, mutated in the sensorineural deafness and vestibular disorder, DFNA9. Hum Mol Genet 2001;10:2493-2500.

24. Robertson NG, Cremers CWRJ, Huygen PLM, Ikezono T, Krastins B, Kremer H, Kuo SF, Liberman MC, Merchant SN, Miller CE, Nadol JB Jr, Sarracino DA, Verhagen WI, Morton CC. Cochlin immunostaining of inner ear pathologic deposits and proteomic analysis in DFNA9 deafness and vestibular dysfunction. Hum Mol Genet 2006;15:1071-1085.

25. Bischoff AMLC, Pennings RJE, Huygen PLM, Luijendijk MWJ, van Wijk E, Cruysberg JRM, Kremer H, Cremers CWRJ. Cochleovestibular and ocular features in a Dutch DFNA11 family. Otol Neurotol 2006;27:323-331.

26. Fransen E, Verstreken M, Bom SJH, Lemaire F, Kemperman MH, De Kok YJ, Wuyts FL, Verhagen WI, Huygen PL, McGuirt WT, Smith RJ, Van Maldergem LV, Declau F, Cremers CW, Van De Heyning PH, Cremers FP, Van Camp G. A common ancestor for COCH related cochleovestibular (DFNA9) patients in Belgium and The Netherlands bearing the P51S mutation. J Med Genet 2001;38:61-65.

27. Bhattacharya SK, Rockwood EJ, Smith SD, Bonilha VL, Crabb JS, Kuchtey RW, Robertson NG, Peachey NS, Morton CC, Crabb JW. Proteomics reveal cochlin deposits associated with glaucomatous trabecular meshwork. J Biol Chem 2005;280:6080-6084.

28. Bhattacharya SK, Annangudi SP, Salomon RG, Kuchtey RW, Peachey NS, Crabb JW. Cochlin deposits in the trabecular meshwork of the glaucomatous DBA/2J mouse. Exp Eye Res 2005;80:741-744.

29. Pauw RJ, Collin RWJ, Huygen PLM, Hoefsloot LH, Kremer H, Cremers CWRJ. Clinical characteristics of a Dutch DFNA9 family with a novel COCH mutation, G87W. Audiol Neurootol 2007;12:77-84.

30. Khetarpal U, Schuknecht HF, Gacek RR, Holmes LB. Autosomal dominant sensorineural hearing loss. Pedigrees, audiologic findings, and temporal bone findings in two kindreds. Arch Otolaryngol Head Neck Surg 1991;117:1032-1042.

31. Khetarpal U. Autosomal dominant sensorineural hearing loss: further temporal bone findings. Arch Otolaryngol Head Neck Surg 1993;119:106-108.

32. Merchant SN, Linthicum FH, Nadol JB. Histopathology of the inner ear in DFNA9. In: Kitamura K, Steel KP (eds). Genetics in Otorhinolaryngology. Advances Oto-RhinoLaryngology, vol. 56. Basel: Karger, 2000;56:212-217.

33. Khetarpal U. DFNA9 is a progressive audiovestibular dysfunction with a microfibrillar deposit in the inner ear. Laryngoscope 2000;110:1379-1384.

34. Bhattacharya SK. Focus on molecules: cochlin. Exp Eye Res 2006;82:355-356.

35. Bron AJ. Anterior Corneal Mosaic. Br J Ophthalmol 1968;52:659-669.

36. Bron AJ, Tripathi RC. Anterior Corneal Mosaic. Further observations. Br J Ophthalmol 1969; 53:760-764.

37. Bron AJ, Tripathi RC. Anterior Corneal Mosaic. Br J Physiol Opt 1970;25:8-13.

38. Bron AJ. Photography of corneal pattern. Arch Ophthalmol 1968;79:119-120.

39. Bron AJ. The architecture of the corneal stroma. Br J Ophthalmol 2001;85:379-381.

40. Norn MS. Schweitzer's polygonal fluorescein pattern of the cornea. A clinical study and comparison with an applanation-prism-net-phenomenon. Acta Ophthalmol 1968;46:700-711. 


\section{APPENDIX}

Results of $\mathrm{COCH}$ Mutation Analysis, Corneal Findings and Ophthalmologic Examination in Four DFNA9 Families

\section{Family 1}

\begin{tabular}{|c|c|c|c|c|c|c|c|c|c|c|c|c|c|}
\hline \multicolumn{2}{|c|}{$\begin{array}{l}\text { Patient } \\
\text { Code }\end{array}$} & \multirow{3}{*}{$\begin{array}{l}\text { Gender } \\
\text { Age (y) } \\
\text { F, } 18\end{array}$} & \multirow{3}{*}{$\begin{array}{l}\text { Mutation } \\
\\
-\end{array}$} & \multirow{2}{*}{\multicolumn{2}{|c|}{$\begin{array}{l}\text { Visual } \\
\text { acuity } \\
\text { RE LE }\end{array}$}} & \multirow{2}{*}{ Cornea } & \multicolumn{2}{|c|}{$\begin{array}{l}\text { Keratometry } \\
(\mathrm{mm})\end{array}$} & \multicolumn{2}{|c|}{$\begin{array}{l}\text { Schirmer } \\
(\mathrm{mm} 5 \\
\text { min) }\end{array}$} & \multicolumn{2}{|c|}{$\begin{array}{l}\text { IOP } \\
(\mathrm{mm} \mathrm{Hg})\end{array}$} & \multirow[t]{2}{*}{ Other findings } \\
\hline & & & & & & & RE & LE & RE & LE & RE & LE & \\
\hline 1 & $1 . \mathrm{V9}$ & & & 0.9 & 0.9 & Normal & 7,42 & 7,42 & & & & & Congenital cataract \\
\hline 2 & 1.V12 & M, 19 & - & 1.0 & 1.0 & Normal & 7,51 & 7,43 & & & & & \\
\hline 3 & $1 . \mathrm{V} 4$ & $\mathrm{~F}, 23$ & + & 1.0 & 1.0 & Corneal striae & & & & & & & Dry eyes \\
\hline 4 & $1 . \mathrm{V} 3$ & M, 25 & + & 1.0 & 1.0 & Corneal striae & & & & & & & $\begin{array}{l}\text { Dry eyes, hard contact } \\
\text { lenses }\end{array}$ \\
\hline 5 & $1 . \mathrm{V7}$ & M, 26 & + & 1.0 & 1.0 & Normal & 7,61 & 7,62 & & & & & \\
\hline 6 & $1 . \mathrm{V} 6$ & $\mathrm{~F}, 27$ & + & 1.0 & 1.0 & Normal & & & & & & & \\
\hline 7 & 1.V13 & $\mathrm{F}, 29$ & - & 1.0 & 1.0 & Normal & 7,88 & 7,89 & & & & & Hard contact lenses \\
\hline 8 & $1 . \mathrm{V} 8$ & M, 30 & + & 1.0 & 1.0 & Normal & & & & & & & \\
\hline 9 & 1.V1 & $\mathrm{F}, 31$ & + & 1.0 & 1.0 & Normal & 7,49 & 7,55 & & & & & Refraction instability \\
\hline 10 & 1.V711 & $\mathrm{F}, 38$ & - & 1.0 & 1.0 & Normal & & & & & & & \\
\hline 12 & $1 . \mathrm{V} 25$ & M, 42 & + & 1.0 & 1.2 & Normal & 8,28 & 8,30 & & & 13 & 15 & \\
\hline 13 & $1 . \mathrm{V} 5$ & $\mathrm{~F}, 43$ & + & 1.0 & 1.0 & Normal & & & & & & & \\
\hline 14 & 1.IV24 & M, 45 & + & 1.0 & 1.0 & Normal & & & & & & & \\
\hline 15 & 1.IV12 & $\mathrm{F}, 47$ & + & 1.0 & 1.0 & Normal & 8,00 & 7,92 & 22 & 19 & 16 & 16 & \\
\hline 16 & 1.IV3 & M, 48 & + & 1.0 & 1.0 & Corneal striae & & & & & & & \\
\hline 17 & 1.IV28 & M, 48 & - & 1.0 & 1.0 & Normal & & & & & & & \\
\hline 18 & 1.IV31 & M, 49 & - & 1.0 & 0.8 & Corneal striae & & & & & & & Dry eyes \\
\hline 19 & 1.IV7 & $\mathrm{F}, 50$ & + & 1.0 & 0.8 & Corneal striae & 7,48 & 7,81 & & & & & Incipient cataract \\
\hline 20 & 1.IV18 & $\mathrm{F}, 50$ & + & & & Corneal striae & & & & & & & \\
\hline 21 & 1.IV32 & $\mathrm{F}, 52$ & - & 1.0 & 1.0 & Normal & 7,70 & 7,76 & 21 & 25 & & & Cornea guttata \\
\hline 22 & 1.IV30 & M, 53 & - & & & Corneal striae & & & & & & & \\
\hline 23 & 1.IV29 & M, 54 & - & 1.0 & 0.8 & Corneal striae & & & & & 14 & 13 & Dry eyes \\
\hline 24 & 1.IV21 & M, 54 & + & 1.0 & 1.0 & Corneal striae & 7,63 & 7,83 irr & & & & & \\
\hline 25 & $1 . I V 6$ & $\mathrm{~F}, 54$ & + & 0.8 & 0.9 & Corneal striae & 7,46 & 7,31 & 0 & 2 & 15 & 16 & $\begin{array}{l}\text { Dry eyes, refraction } \\
\text { instability }\end{array}$ \\
\hline 27 & 1.IV4 & $\mathrm{F}, 56$ & + & 1.0 & 1.0 & Corneal striae & 7,39 & 7,36 irr & 8 & 7 & & & Refraction instability \\
\hline 28 & 1.IV1 & M, 57 & + & 0.8 & 0.8 & Corneal striae & 7,28 & 7,43 & 10 & 11 & & & $\begin{array}{l}\text { Refraction instability, } \\
\text { corneal Stahli line }\end{array}$ \\
\hline 29 & 1.IV19 & M, 60 & + & & & Corneal striae & & & & & & & $\begin{array}{l}\text { Cornea guttata, } \\
\text { cataract surgery }\end{array}$ \\
\hline 30 & 1.IV10 & $\mathrm{F}, 61$ & + & & & Corneal striae & & & 9 & 6 & & & Strabismus surgery \\
\hline 31 & 1.IV8 & M, 70 & + & & & Corneal striae & & & 5 & 6 & 18 & 16 & \\
\hline 32 & 1.III8 & M, 72 & + & 0.6 & 0.8 & Corneal striae & & & 2 & 5 & 18 & 18 & \\
\hline 33 & 1.III21 & $\mathrm{F}, 76$ & - & 1.3 & 0.1 & Corneal striae & & & & & & & Venous occlusion LE \\
\hline 34 & 1.III17 & $\mathrm{F}, 77$ & + & 0.8 & 0.8 & Corneal striae & & & 25 & 25 & 18 & 18 & Dry eyes \\
\hline
\end{tabular}

$\mathrm{IOP}=$ intraocular pressure; $\mathrm{RE}=$ right eye; $\mathrm{LE}=$ left eye; irr = irregular; Visual acuity = best corrected visual acuity. 
Family 2

\begin{tabular}{|c|c|c|c|c|c|c|c|c|c|c|c|c|c|}
\hline \multirow{2}{*}{\multicolumn{2}{|c|}{$\begin{array}{l}\text { Patient } \\
\text { Code }\end{array}$}} & \multirow{4}{*}{$\begin{array}{l}\text { Gender } \\
\text { Age (y) } \\
\text { M, } 41\end{array}$} & \multirow{4}{*}{$\begin{array}{l}\text { Mutation } \\
-\end{array}$} & \multirow{2}{*}{\multicolumn{2}{|c|}{$\begin{array}{l}\text { Visual } \\
\text { acuity }\end{array}$}} & \multirow{4}{*}{$\begin{array}{l}\text { Cornea } \\
\text { Normal }\end{array}$} & \multirow{2}{*}{\multicolumn{2}{|c|}{$\begin{array}{l}\text { Keratometry } \\
(\mathrm{mm})\end{array}$}} & \multicolumn{2}{|c|}{$\begin{array}{l}\text { Schirmer } \\
(\mathrm{mm} 5 \\
\min )\end{array}$} & \multicolumn{2}{|c|}{$\begin{array}{l}\text { IOP } \\
(\mathrm{mm} \mathrm{Hg})\end{array}$} & \multirow[t]{2}{*}{ Other findings } \\
\hline & & & & & & & & & & & & & \\
\hline & & & & \multirow{2}{*}{$\frac{\mathrm{RE}}{1.0}$} & \multirow{2}{*}{$\frac{\mathrm{LE}}{1.0}$} & & \multirow{2}{*}{$\frac{\mathrm{RE}}{7,92}$} & \multirow{2}{*}{$\frac{\mathrm{LE}}{7,88}$} & \multirow{2}{*}{ RE } & \multirow{2}{*}{ LE } & \multirow{2}{*}{ RE } & \multirow{2}{*}{ LE } & \\
\hline 1 & 2.IV86 & & & & & & & & & & & & \\
\hline 2 & 2.IV54 & $\mathrm{F}, 44$ & + & 1.0 & 1.0 & Normal & & & & & & & Dry eyes \\
\hline 3 & 2.IV3 & $\mathrm{F}, 46$ & - & 1.0 & 1.0 & Normal & 7,74 & 7,74 & 17 & 17 & 17 & 17 & \\
\hline 4 & 2.IV50 & $\mathrm{M}, 47$ & + & 1.0 & 1.0 & Corneal striae & 7,74 & $7,68 \mathrm{irr}$ & & & & & \\
\hline 5 & 2.IV2 & $\mathrm{F}, 48$ & - & 0.3 & 1.0 & Normal & 7,82 & 7,78 & 5 & 5 & 12 & 12 & $\begin{array}{l}\text { Anisometropic } \\
\text { amblyopia RE }\end{array}$ \\
\hline 6 & 2.IV52 & M, 49 & - & 1.0 & 1.0 & Normal & 8,07 & 8,06 & 33 & 11 & 14 & 16 & \\
\hline 7 & 2.IV48 & M, 49 & + & 0.8 & 0.9 & Corneal striae & & & & & & & Dry eyes \\
\hline 8 & 2.IV39 & M, 49 & + & 1.0 & 1.0 & Normal & 7,74 & 7,68 & & & & & \\
\hline 9 & 2.IV6 & $\mathrm{F}, 50$ & - & 1.0 & 1.0 & Normal & 7,49 & 7,52 & 25 & 20 & 14 & 15 & \\
\hline 10 & 2.IV46 & $F, 52$ & + & 0.5 & 0.5 & Corneal striae & 7.60 & 7.79 & & & & & $\begin{array}{l}\text { Astigmatism, } \\
\text { exophoria }\end{array}$ \\
\hline 11 & 2.IV4 & $\mathrm{M}, 54$ & - & 1.0 & 1.0 & Normal & 7,50 & 7,53 & 11 & 14 & 20 & 20 & \\
\hline 12 & 2.IV29 & $\mathrm{F}, 55$ & + & 0.9 & 0.9 & Corneal striae & 7,60 & $7,79 \mathrm{irr}$ & & & & & Corneal arcus \\
\hline 13 & 2.IV44 & $\mathrm{M}, 57$ & - & 1.0 & 1.0 & Normal & 7,82 & 7,75 & 13 & 15 & 15 & 15 & \\
\hline 14 & 2.IV42 & $M, 58$ & - & 1.0 & 1.0 & Normal & 7,84 & 7,85 & 0 & 1 & 12 & 12 & \\
\hline 15 & 2.III43 & $\mathrm{F}, 73$ & + & 0.5 & 0.6 & Corneal striae & & & & & & & Dry eyes \\
\hline 16 & 2.III33 & $\mathrm{M}, 74$ & + & 0.6 & 0.6 & Corneal striae & irreg & dlar & & & & & Refraction instability \\
\hline 17 & 2.III24 & $\mathrm{M}, 74$ & + & & & Corneal striae & 7,56 & 7.56 & & & & & Incipient cataract \\
\hline 18 & 2.III2 & $\mathrm{M}, 80$ & + & 0.5 & 0.8 & Normal & 7,71 & 7,90 & 28 & 26 & 17 & 14 & $\begin{array}{l}\text { Cataract surgery RLE, } \\
\text { retinal detachment } \\
\text { surgery RE, ectropion } \\
\text { surgery RE }\end{array}$ \\
\hline
\end{tabular}

IOP, intraocular pressure; RE, right eye; LE, left eye; irr, irregular; Visual acuity = best corrected visual acuity. 
Chapter 3.2

Family 3

\begin{tabular}{|c|c|c|c|c|c|c|c|c|c|c|c|c|c|}
\hline \multicolumn{2}{|c|}{$\begin{array}{l}\text { Patient } \\
\text { Code }\end{array}$} & \multirow[t]{2}{*}{$\begin{array}{l}\text { Gender } \\
\text { Age (y) }\end{array}$} & \multirow[t]{2}{*}{ Mutation } & \multicolumn{2}{|c|}{$\begin{array}{l}\text { Visual } \\
\text { acuity }\end{array}$} & \multirow[t]{2}{*}{ Cornea } & \multicolumn{2}{|c|}{$\begin{array}{l}\text { Keratometry } \\
(\mathrm{mm})\end{array}$} & \multicolumn{2}{|c|}{$\begin{array}{l}\text { Schirmer } \\
\text { (mm } 5 \mathrm{~min})\end{array}$} & \multicolumn{2}{|c|}{$\begin{array}{l}\text { IOP } \\
(\mathrm{mm} \mathrm{Hg})\end{array}$} & \multirow[t]{2}{*}{ Other findings } \\
\hline & & & & RE & LE & & RE & LE & RE & LE & RE & LE & \\
\hline 1 & 3.IV13 & M, 28 & - & 1.0 & 1.0 & Normal & 7,58 & 7,70 & 15 & 15 & 14 & 14 & Retinal detachment \\
\hline 2 & 3.IV14 & M, 30 & + & 1.0 & 1.0 & Normal & & & & & & & \\
\hline 3 & 3.IV24 & $\mathrm{F}, 33$ & + & 1.0 & 1.0 & Normal & 7,63 & 7,73 & 15 & 15 & 14 & 14 & \\
\hline 4 & 3.III32 & $\mathrm{F}, 48$ & + & 1.0 & 1.0 & Corneal striae & & & & & & & \\
\hline 5 & 3.III21 & M, 50 & - & 1.0 & 1.0 & Normal & & & & & & & \\
\hline 6 & 3.III19 & M, 51 & + & 0.6 & 0.6 & Corneal striae & 7,84 & 7,53 & 15 & 15 & 15 & 15 & $\begin{array}{l}\text { Cataract, } \\
\text { astigmatism LE }\end{array}$ \\
\hline 7 & 3.III16 & M, 55 & + & 1.0 & 1.0 & Normal & & & & & & & \\
\hline 8 & 3.III30 & M, 55 & - & 1.0 & 1.0 & Corneal striae & 7,92 & 7,90 & 2 & 2 & 15 & 15 & \\
\hline 9 & 3.III26 & $\mathrm{F}, 55$ & + & 1.0 & 1.0 & Normal & 7,52 & 7,52 & 15 & 15 & 14 & 14 & \\
\hline 10 & 3.III15 & M, 56 & + & 1.0 & 1.0 & Corneal striae & & & & & & & \\
\hline 11 & 3.III11 & $\mathrm{F}, 57$ & - & 1.0 & 0.1 & Normal & 7,55 & 7,59 & 25 & 20 & 19 & 20 & $\begin{array}{l}\text { Anisometropicopia, } \\
\text { retinal laser } \\
\text { coagulation }\end{array}$ \\
\hline 12 & 3.III6 & M, 58 & - & 1.0 & 1.0 & Normal & 7,81 & 7,79 & 12 & 25 & 12 & 12 & \\
\hline 13 & 3.III8 & M, 59 & + & 0.6 & 0.6 & Corneal striae & 7,40 & 7,51 & 2 & 2 & 15 & 15 & Myopic fundus \\
\hline 14 & 3.III3 & $F, 63$ & - & 1.0 & 1.0 & Normal & 7,43 & 7,48 & 13 & 7 & 18 & 18 & $\begin{array}{l}\text { Asteroid } \\
\text { hyelopathy, iris cyst } \\
\text { RE }\end{array}$ \\
\hline 15 & 3.III1 & $\mathrm{F}, 65$ & - & 1.0 & 1.0 & Normal & 8,11 & 8,20 & 20 & 22 & 14 & 14 & \\
\hline 16 & 3.II16 & M, 79 & + & 1.0 & 1.0 & Normal & & & 18 & 23 & & & \\
\hline
\end{tabular}

IOP, intraocular pressure; RE, right eye; LE, left eye; Visual acuity = best corrected visual acuity. 
Family 4

\begin{tabular}{|c|c|c|c|c|c|c|c|c|c|}
\hline \multicolumn{2}{|c|}{$\begin{array}{l}\text { Patient } \\
\text { Code }\end{array}$} & \multirow[t]{2}{*}{$\begin{array}{l}\text { Gender } \\
\text { Age (y) }\end{array}$} & \multirow[t]{2}{*}{ Mutation } & \multicolumn{2}{|c|}{$\begin{array}{l}\text { Visual } \\
\text { acuity }\end{array}$} & \multirow[t]{2}{*}{ Cornea } & \multicolumn{2}{|c|}{$\begin{array}{l}\text { IOP } \\
(\mathrm{mm} \mathrm{Hg})\end{array}$} & \multirow[t]{2}{*}{ Other findings } \\
\hline & & & & RE & LE & & RE & LE & \\
\hline 1 & 4.IV38 & F, 26 & + & 1.0 & 1.0 & Normal & 14 & 14 & \\
\hline 2 & 4.IV82 & M, 29 & + & 1.0 & 1.0 & Normal & 13 & 13 & \\
\hline 3 & 4.IV81 & $\mathrm{F}, 30$ & + & 1.0 & 1.0 & Normal & 12 & 12 & Dry eyes \\
\hline 4 & 4.IV83 & M, 32 & + & 1.0 & 1.0 & Normal & 12 & 12 & \\
\hline 5 & 4.IV80 & F, 33 & - & 1.0 & 1.0 & Normal & 12 & 12 & \\
\hline 6 & 4.IV31 & M, 34 & - & 1.0 & 1.0 & Normal & 13 & 12 & Exophoria \\
\hline 7 & 4.IV21 & $F, 36$ & + & 1.0 & 1.0 & Normal & 10 & 10 & \\
\hline 8 & 4.IV27 & F, 39 & + & 1.0 & 1.0 & Normal & 12 & 12 & \\
\hline 9 & 4.IV43 & $F, 39$ & - & 1.0 & 1.0 & Normal & 10 & 10 & \\
\hline 10 & 4.IV18 & M, 39 & + & 1.0 & 1.0 & Normal & 12 & 13 & \\
\hline 11 & 4.IV17 & $\mathrm{F}, 40$ & + & 1.0 & 1.0 & Normal & 13 & 13 & \\
\hline 12 & 4.IV16 & $\mathrm{F}, 41$ & - & 1.0 & 1.0 & Normal & 16 & 14 & \\
\hline 13 & 4.IV20 & M, 34 & + & 1.0 & 1.0 & Normal & 11 & 11 & \\
\hline 14 & 4.IV19 & $\mathrm{F}, 44$ & - & 1.0 & 1.0 & Normal & 11 & 11 & \\
\hline 15 & 4.III83 & M, 55 & + & 1.0 & 1.0 & Normal & 12 & 12 & Dry eyes \\
\hline 16 & 4.III32 & $\mathrm{F}, 56$ & + & 0.8 & 0.8 & Normal & 15 & 15 & \\
\hline 17 & 4.III30 & $\mathrm{F}, 58$ & - & 1.0 & 1.0 & Normal & 12 & 12 & \\
\hline 18 & 4.III72 & M, 59 & + & 1.0 & 0.2 & Normal & 12 & 13 & Dry eyes, bullous keratopathy \\
\hline 19 & 4.III81 & $\mathrm{F}, 59$ & + & 1.0 & 0.8 & Normal & 10 & 10 & Dry eyes, exophthalmos (Graves) \\
\hline 20 & 4.III25 & $\mathrm{F}, 62$ & - & 1.0 & 1.0 & Normal & 13 & 13 & Dry eyes \\
\hline 21 & 4.III53 & M, 63 & - & 0.6 & 1.0 & Normal & 14 & 13 & Incipient cataract \\
\hline 22 & 4.III77 & $\mathrm{F}, 63$ & - & 1.0 & 1.0 & Normal & 14 & 14 & Dry eyes \\
\hline 23 & 4.III71 & $\mathrm{F}, 64$ & + & 0.3 & 0.8 & Corneal striae RE & 12 & 15 & $\begin{array}{l}\text { Dry eyes, keratoconus, cataract } \\
\text { surgery LE, corneal } \\
\text { transplantation LE }\end{array}$ \\
\hline 24 & 4.III20 & M, 67 & + & 1.0 & 0.8 & Normal & 10 & 10 & Strabismus (esotropia) LE \\
\hline 25 & 4.III49 & M, 68 & + & 1.0 & 0.8 & Normal & 12 & 10 & \\
\hline 26 & 4.III39 & M, 69 & + & 0.8 & 1.0 & Normal & 12 & 12 & \\
\hline 27 & 4.III18 & M, 70 & - & 0.8 & 0.8 & Normal & 12 & 12 & $\begin{array}{l}\text { Retinal detachment surgery RE, } \\
\text { cataract surgery }\end{array}$ \\
\hline 28 & 4.III16 & M, 71 & + & 0.8 & 1.0 & Normal & 12 & 13 & Incipient cataract \\
\hline 29 & 4.III 45 & $\mathrm{~F}, 72$ & + & 0.6 & 1.0 & Normal & 16 & 16 & Incipient cataract \\
\hline 30 & 4.III12 & M, 74 & - & 1.0 & 1.0 & Normal & 14 & 12 & Incipient cataract \\
\hline
\end{tabular}

IOP, intraocular pressure; RE, right eye; LE, left eye; Visual acuity = best corrected visual acuity. 


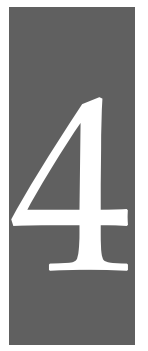

DFNA11 



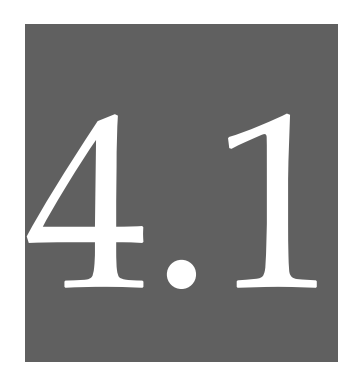

\title{
Cochleovestibular and ocular features in a Dutch DFNA11
}

family

\author{
A.M.L.C. Bischoff \\ R.J.E. Pennings \\ P.L.M. Huygen \\ M.W.J. Luijendijk \\ E. van Wijk \\ J.R.M. Cruysberg \\ H. Kremer \\ C.W.R.J. Cremers
}





\section{Abstract}

Objectives: To report hearing impairment and vestibular and ocular features in a Dutch DFNA11 family and to compare these results to reported data on three other DFNA11 families.

Study Design: Family study.

Methods: Regression analysis was performed in relation to age to outline the development of hearing thresholds and speech recognition scores. Vestibular and ocular functions were examined.

Results: First symptoms of hearing impairment started between the ages of four and 43 years. Most of the audiograms were symmetric and flat or downsloping. The annual threshold deterioration increased from 0.2 to $2.6 \mathrm{~dB}$ per year at 0.25 to $8 \mathrm{kHz}$ in the longitudinal analyses and in the cross-sectional analysis from 0.3 to $0.9 \mathrm{~dB}$ per year. The speech recognition score was quite good, deteriorating by $0.9 \%$ per year from a $90 \%$ score at the age of 36 years onwards. Remarkably, extensive ocular examination including corrected visual acuity and refraction measurements, slit-lamp examination, ophthalmoscopy, Goldmann perimetry, electroretinography and electro-oculography revealed signs of subclinical retinal dysfunction. None of the patients showed the classic triad of retinitis pigmentosa. Pure-tone thresholds, phoneme recognition scores and vestibular responses of the mutation carriers were fairly similar to previously described DFNA11 families.

Conclusion: Even though the diverse mutations are located in different regions of the myosin VIIa gene, the cochleovestibular phenotype is fairly similar in all DFNA11 families. Surprisingly, subclinical retinal dysfunction was detected only in this family.

\section{Introduction}

Since about 15 years, linkage and mutation analysis have been used extensively to gain insight into the causes of hereditary non-syndromic hearing impairment. Genetic hearing impairment is heterogeneous. Nowadays, more than 50 loci and 21 genes are known for non-syndromic autosomal dominant forms of hearing impairment (DFNA). Also, 57 loci and 21 genes have been identified for autosomal recessive forms (DFNB). ${ }^{1}$ In some cases, the same gene is responsible for syndromic as well as non-syndromic hearing impairment, such as WFS1 for Wolfram syndrome ${ }^{2}$ and DFNA6/14/38.,3 Other genes are involved in both autosomal dominant and autosomal recessive hearing impairment. Mutations in the TECTA gene, for example, can cause either DFNA8/12 or DFNB21.5,6 
Mutations in the myosin VIIa (MYO7A) gene cause non-syndromic autosomal dominant (DFNA11) ${ }^{7,8}$ and autosomal recessive (DFNB2) hearing impairment, ${ }^{9,10}$ Usher syndrome Type 1B (USH1B) ${ }^{11}$ and atypical Usher syndrome. ${ }^{12,13}$ MYO7A encodes the protein myosin VIIa and is expressed in the inner ear, retina, testis, lung, kidney, liver, olfactory epithelium and intestine. ${ }^{14-16}$ Expression is more prominent in inner hair cells than in outer hair cells of the cochlea, where the protein is located in the stereocilia, the cell body and the cuticular plate. ${ }^{14}$ Myosin VIIa is also present in Type I and Type II hair cells of the utricle and semicircular canals. In the eye, this protein has been found in the retinal pigment epithelial cells and in photoreceptor cells, mainly in the inner, the base of the outer segments and the synaptic endings. ${ }^{17}$ In addition, Liu et al. identified myosin VIIa to be situated in the connecting cilium of photoreceptor cells. ${ }^{18}$ Myosin VIIa is an unconventional myosin motor molecule that moves along actin filaments using actin-activated ATP-ase activity for its force and motion. It is involved in the organization of the cochlear hair cell stereocilia and is essential for structural integrity of hair bundles..$^{15} \mathrm{It}$ is also important in the distribution of melanosomes in the retinal pigment epithelial cells ${ }^{19}$ and opsin transport in photoreceptor cells ${ }^{20}$ and is part of the Usher I protein complex. ${ }^{21,22}$

Four DFNA11 families have currently been described. ${ }^{23-27}$ Recently a fifth DFNA11 family with a 699 C-to-T transition in MYO7A which leads to an Ala230Val substitution in the motor domain, was presented by poster..$^{28}$ The present report describes the results of audiovestibular and ocular examinations of affected family members in the Dutch DFNA11 family. Luijendijk et al. previously reported on an Asn458Ile mutation in the motor domain of myosin VIIa that causes DFNA11 in this family. ${ }^{25}$ Pure-tone and speech audiometric data were analysed and compared with the results described for the other DFNA11 families. ${ }^{23,24,26,27}$

\section{Patients and Methods}

\section{Patients}

Twenty-six individuals of the Dutch DFNA11 family (W02-011) participated in this study. Signed informed consent was obtained from all participants. The study was approved by the local medical ethics committee. Medical history was taken, with emphasis on hearing impairment and vestibular and ocular symptoms. Nonhereditary causes of hearing impairment were excluded.

Blood samples were obtained for linkage and mutation analysis. Guided by the phenotypic features, linkage analysis for DFNA11 confirmed linkage to the locus of MYO7A. Mutation analysis of MYO7A revealed a c.1373A>T nucleotide 
substitution in exon 13, which is predicted to cause an Asn458Ile amino acid substitution in the motor domain of myosin VIIa. ${ }^{25}$

\section{Audiovestibular examination and analysis}

All participants underwent otologic examination. Pure-tone and speech audiometry were performed in a sound-treated room, according to common clinical standards. Vestibular examination, carried out as previously described, ${ }^{29}$ was performed in six affected mutation carriers. Binaural mean pure-tone threshold levels were calculated for each frequency. Longitudinal and crosssectional analyses were performed for each frequency separately, using linear regression analysis. Longitudinal analysis covered the cases with sufficient serial audiograms. Cross-sectional analysis included the threshold data of the singlesnapshot measurements as well as the mean threshold-by-age values derived for the longitudinal cases. Slope was called annual threshold deterioration (ATD), expressed in $\mathrm{dB}$ per year. When the $95 \%$ confidence interval for slope did not include zero, slope (or ATD) was labelled as significant. Because the longitudinal regression lines generally could not be pooled according to an analysis similar to analysis of covariance (ANCOVA, Prism PC Version 3; GraphPad, San Diego CA, U.S.A.), a trend line was drawn for each frequency on the basis of median slope and intercept values. The longitudinal and cross-sectional trend lines were used to construct two different sets of age-related typical audiograms (ARTA). ${ }^{30}$ To compare audiologic results between the present and the other DFNA11 families, ARTA were also derived for the Japanese and the American families, using available cross-sectional data. ${ }^{23,26}$

Speech audiometry was performed with a standard phonetically balanced Dutch word list. Maximum monaural phoneme recognition scores were derived from individual performance-versus-intensity plots. Cross-sectional recognition scores averaged for both ears were plotted against age and binaural mean pure-tone average (PTA1,2,4 kHz) in decibels hearing level (HL). Onset age and onset level were identified at a maximum phoneme recognition score of $90 \%$ in the performanceversus-age and performance-versus-impairment plots. The slope was called deterioration rate and deterioration gradient, respectively. ${ }^{31}$ Only speech recognition scores of the Japanese family were available for comparison of speech deterioration between the DFNA11 families. ${ }^{23}$

\section{Ocular examination}

In five clinically affected mutation carriers, ocular examinations consisting of corrected visual acuity and refraction measurements, slit-lamp examination, 
ophthalmoscopy and Goldmann perimetry were performed to detect signs of retinitis pigmentosa. Additional ophthalmologic tests included electroretinography (ERG) and electro-oculography (EOG), which were performed and evaluated according to the International Society for Clinical Electrophysiology of Vision Standards. ${ }^{32,33}$ Intraocular pressure measurements (applanation tonometry) and keratometry readings were also obtained.

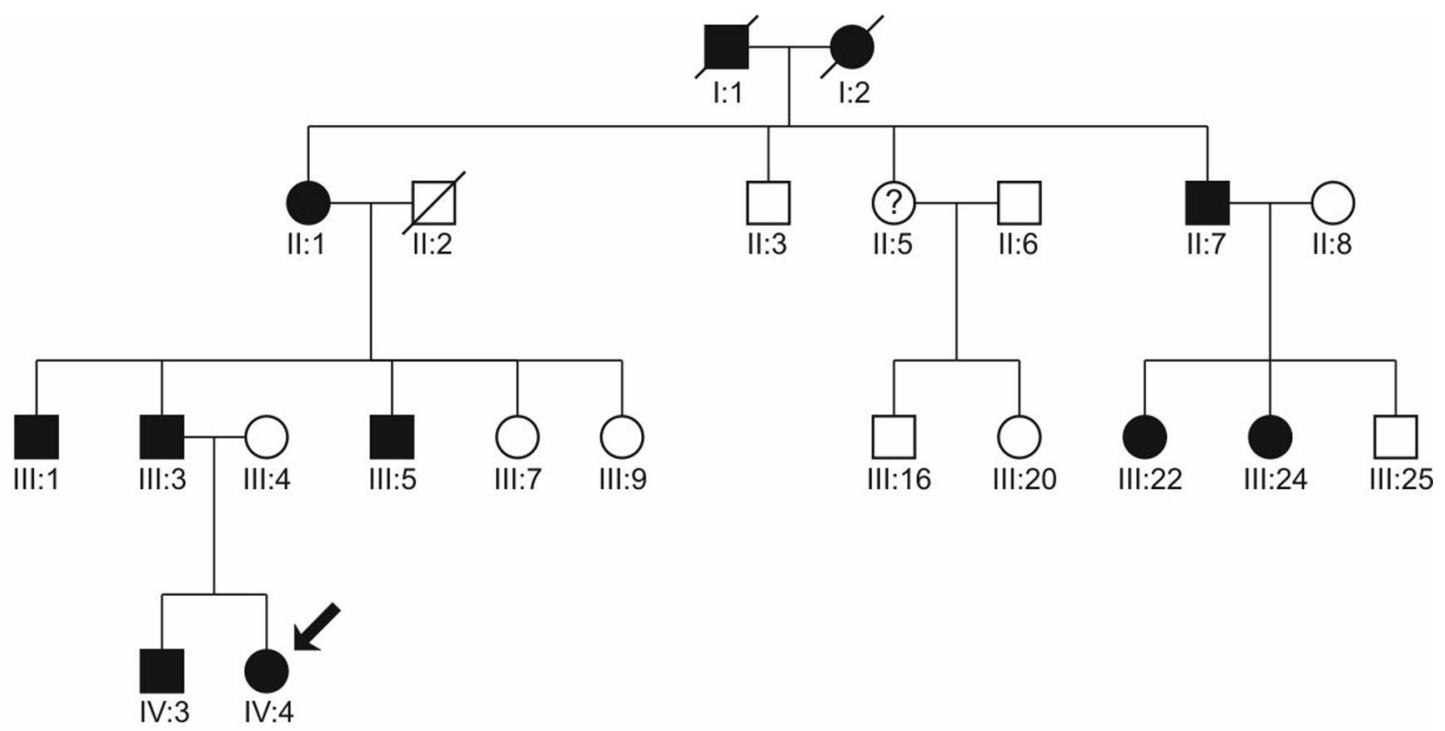

Figure 1. Part of the pedigree of the present DFNA11 family. Square, man; circle, woman; filled symbol, affected person; open symbol, not affected; arrow, proband. The hearing status of individual II:5 was unclear. Mutation analysis showed that this person was not a mutation carrier.

\section{Results}

The pedigree (Figure 1) comprised 11 affected family members. Both spouses in the first-generation marriage had severe hearing impairment, which complicated the genetic analysis. The hearing status of individual II:5 was not completely clear. Audiometric data coincided with the individual $95^{\text {th }}$ percentile threshold values of presbyacusis $\left(\mathrm{P}_{95}\right)^{34}$ Mutation analysis showed that this person was not a mutation carrier. ${ }^{25}$ Except for mild noise exposure in two cases (III:1 and III:5), no other causes of hearing impairment were noticed in this family. First symptoms of hearing impairment were dated between the ages of four and 43 years, whereas affected individual II:7 reported the first symptoms at 63 years of age. 

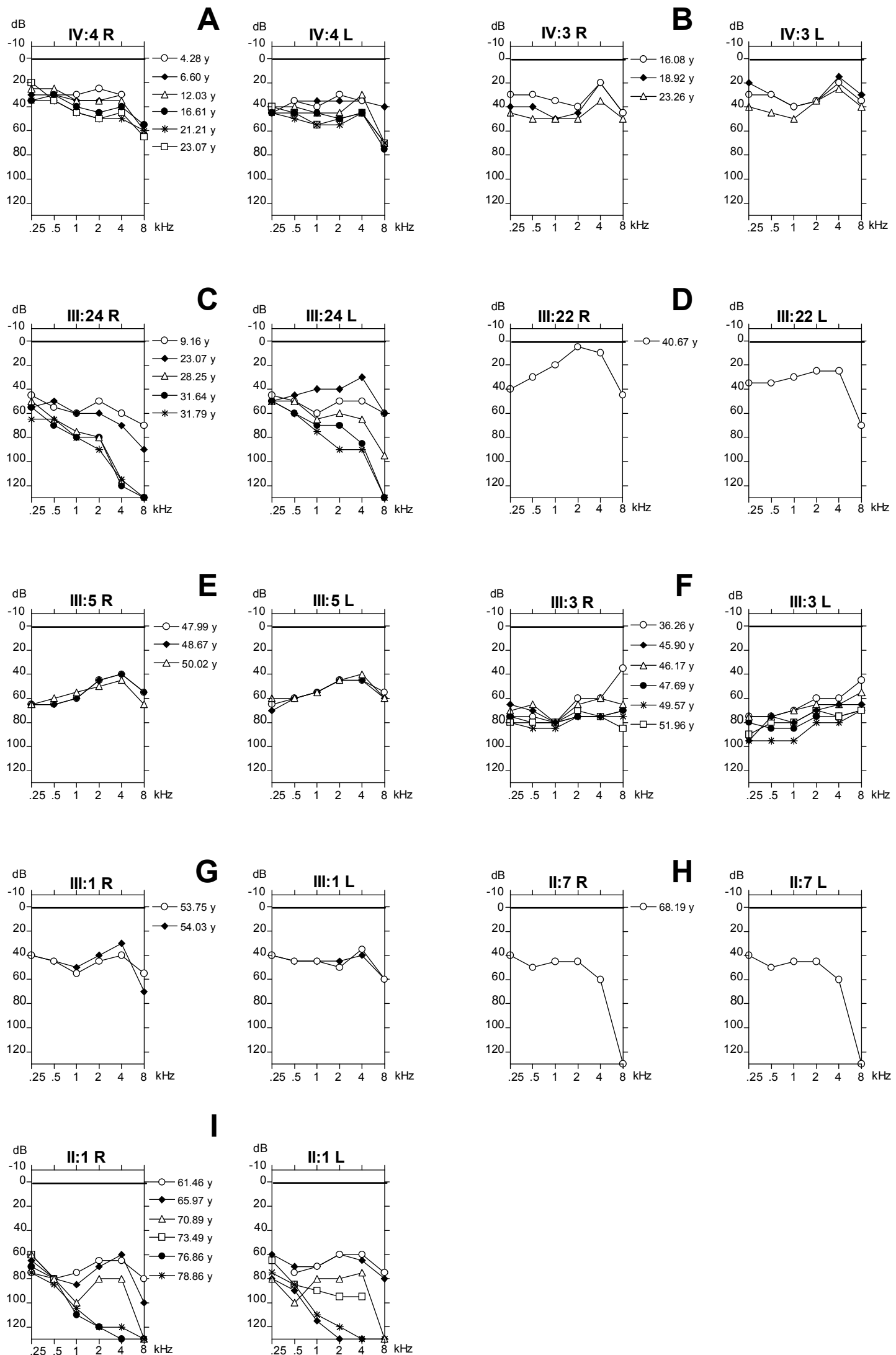

Figure 2 (A-I). Audiograms of the nine affected family members ordered by age at last visit. 
Four persons also reported having tinnitus. Otoscopic examination was normal in all affected individuals. Pure-tone audiograms (Figure 2) were fairly symmetric with moderate to severe hearing loss. The threshold configuration was variable. The pattern was fairly flat at 0.25 to $4 \mathrm{kHz}$ in three individuals (Figure 2B, 2G, $2 \mathrm{H})$, whereas a low-frequency (i.e. a generally ascending configuration) was seen in three other individuals (Figure $2 \mathrm{D}-\mathrm{F}$ ). In the last three individuals it was, or eventually became, gently (Fig 2A) or steeply (Figure 2C, 2I) downsloping. Siblings II:1 and II:7, as well as siblings III:1,3,5 and siblings III:22,24 showed remarkable differences in reported onset age and/or severity of hearing impairment.

\section{Audiometric analysis}

Figure 3 shows longitudinal data of six cases with their regression lines and trend lines. The ATD increased from $0.2 \mathrm{~dB}$ per year $(0.25 \mathrm{kHz})$ to $2.6 \mathrm{~dB}$ per year $(8$ $\mathrm{kHz}$ ). Most of the individual regression lines showed significant progression at the high frequencies. Progression by $2.6 \mathrm{~dB}$ per year at $8 \mathrm{kHz}$ certainly implies progression beyond (median) presbyacusis.

Figure 4 shows a cross-sectional plot of both the three single-snapshot cases and the mean threshold of the longitudinal cases plotted at the mean age for each frequency together with the calculated regression line. Equal weights were thus assigned to all data points, no matter whether they pertained to single-snapshot or longitudinal measurements. The ATD increased from $0.3 \mathrm{~dB}$ per year $(0.25$ $\mathrm{kHz})$ to $0.9 \mathrm{~dB}$ per year $(8 \mathrm{kHz})$. There was no significant progression at any frequency.

Fisher's exact test was used to test for the possible presence of ascertainment bias in the cross-sectional data. Relative to an arbitrary imaginary dividing line (close to the regression line), the counts of data points representing longitudinal or single-snapshot cases were entered in a $2 \times 2$ contingency table. The singlesnapshot measurements represented a cluster of threshold values that were significantly lower than those pertaining to the longitudinal cases, except for 8 $\mathrm{kHz}$. This suggests the presence of an ascertainment bias: subjects who had presented themselves or had been referred as a patient for repeated measurements can be associated with more severe and progressive hearing impairment than subjects who had audiometry only once (Figure 4).

There was an obvious discrepancy between the results obtained from the longitudinal (Figure 3) and the cross-sectional analyses (Figure 4): the former indicated more progression than the latter. 

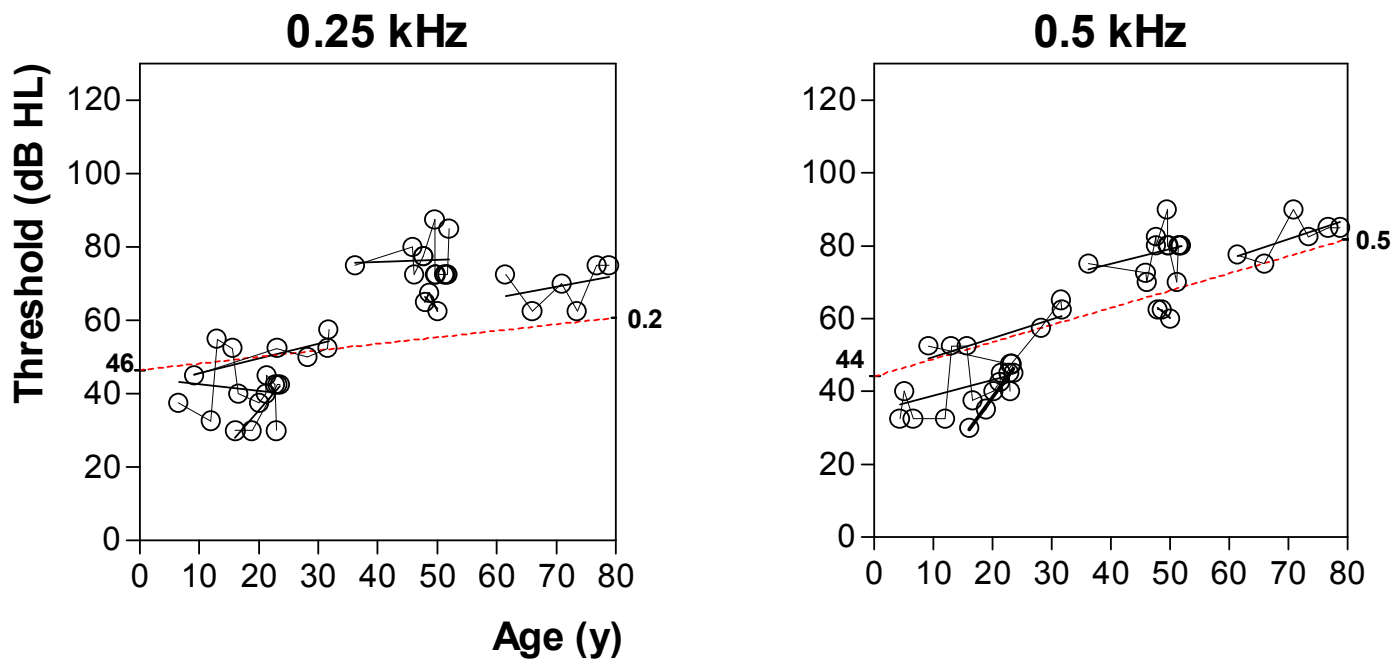

\section{$1 \mathrm{kHz}$}

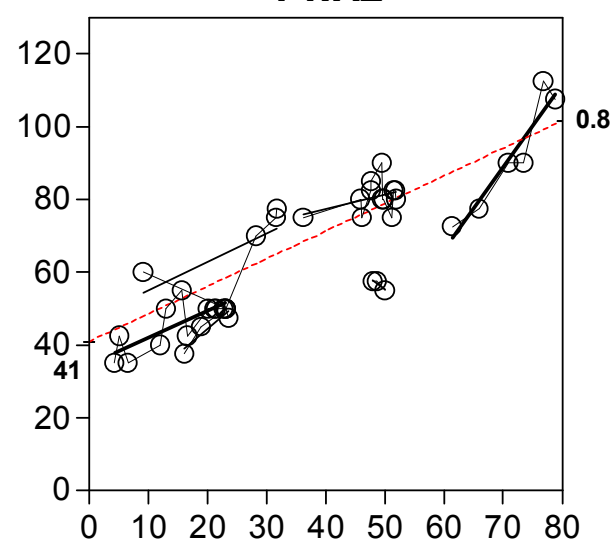

\section{$2 \mathrm{kHz}$}

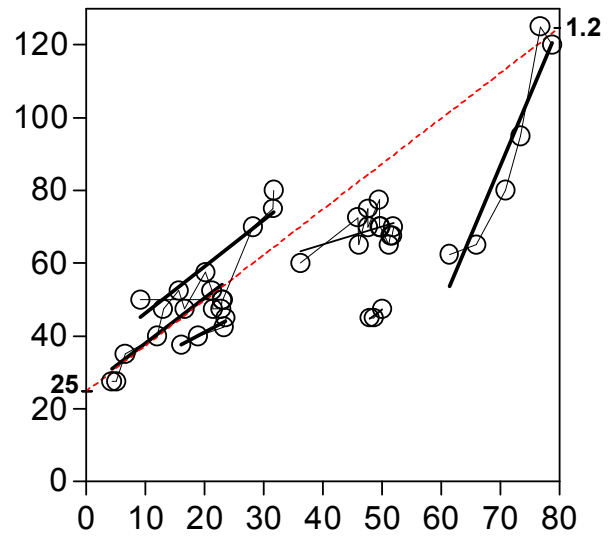

$4 \mathrm{kHz}$

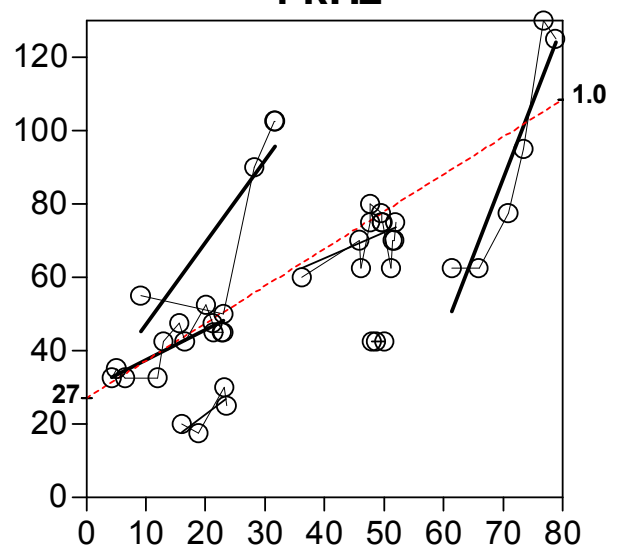

$8 \mathrm{kHz} 2.6$

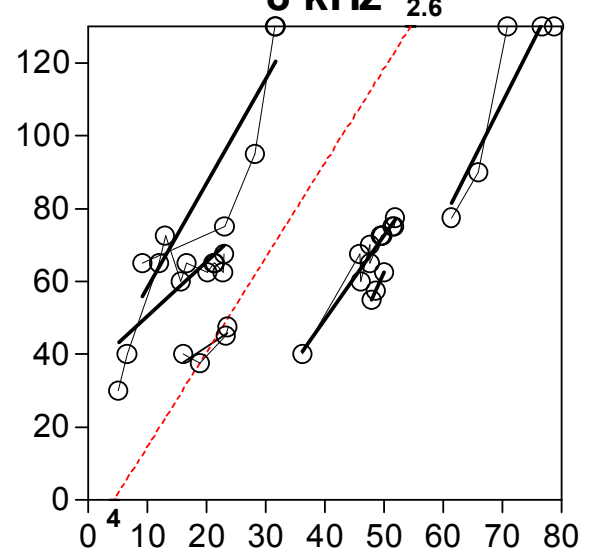

Figure 3. Longitudinal analysis of binaural air conduction thresholds (in decibels hearing level) versus age (in years). Individual longitudinal data (open circles) of six mutation carriers with connecting hairlines are plotted for the frequencies 0.25 to $8 \mathrm{kHz}$. The longitudinal regression lines are included; boldface lines indicate significant progression. The trend lines (dotted) are added to show the ATD per frequency. ATD and intercepts are indicated in boldface figures. 

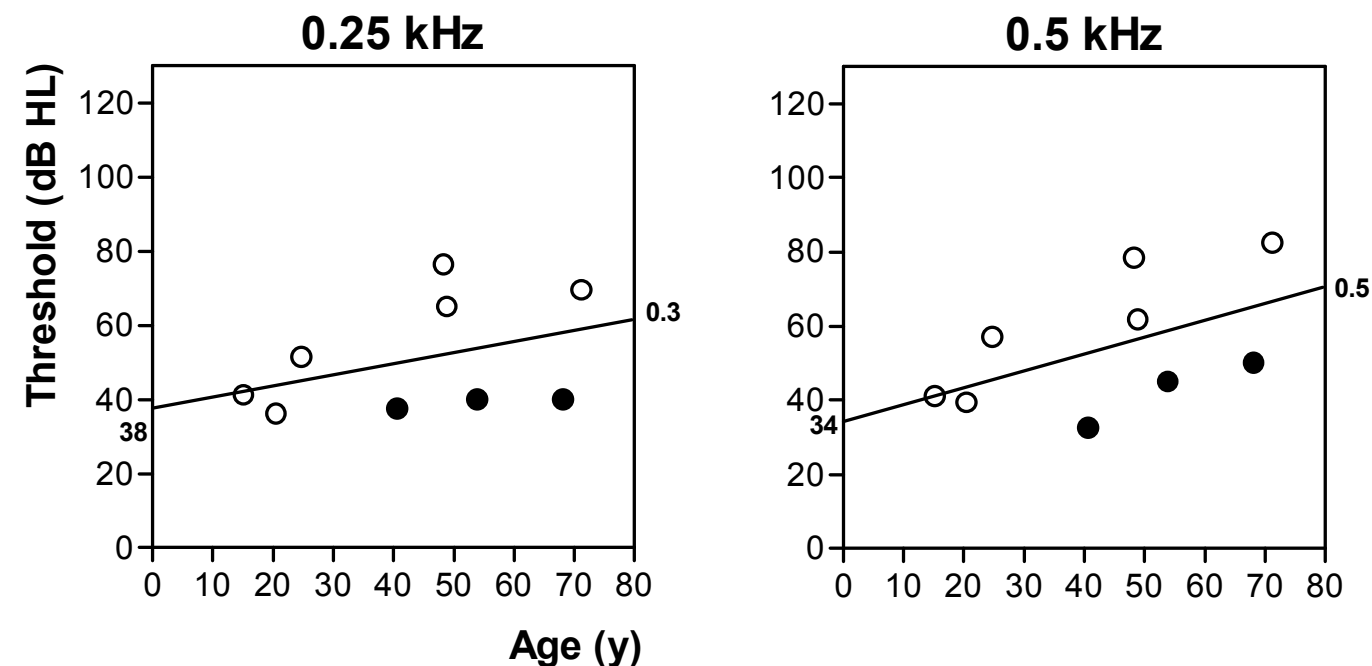

$1 \mathrm{kHz}$

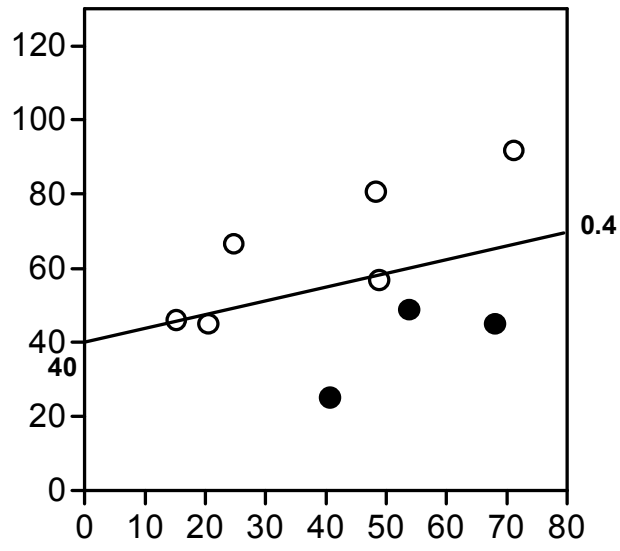

$2 \mathrm{kHz}$

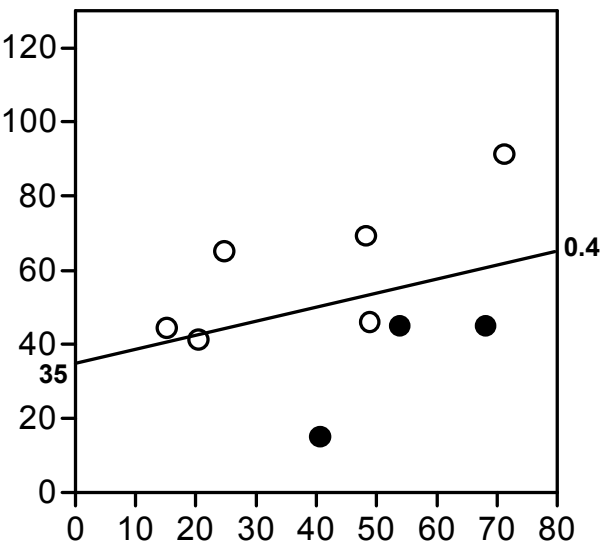

$4 \mathrm{kHz}$

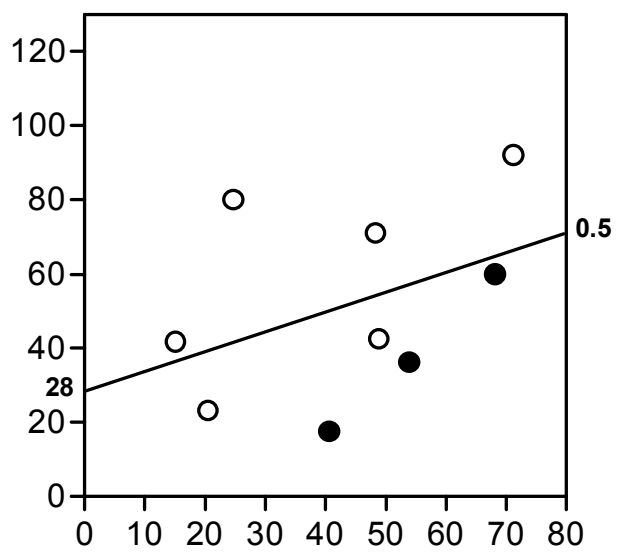

\section{$8 \mathrm{kHz}$}

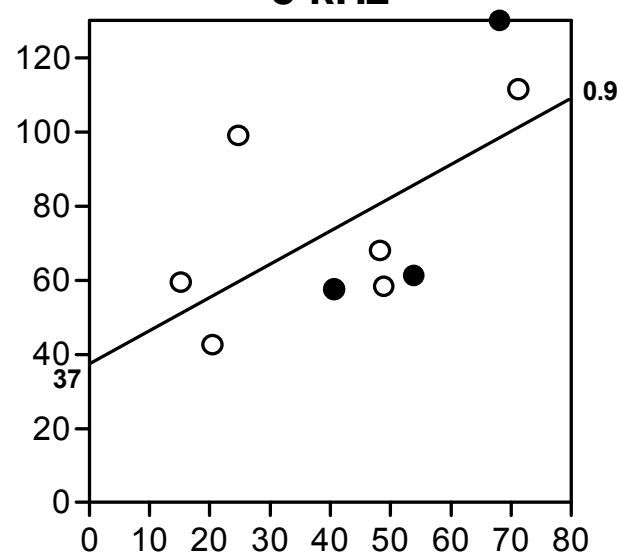

Figure 4. Cross-sectional analysis of the nine mutation carriers of the present family; threshold data of the single-snapshot cases (three cases, filled circles) with the mean threshold and age values derived for the longitudinal cases (six cases, open circles). Regression analysis with ATD and intercepts indicated in boldface type. 
This was attributed to inclusion of the single snapshots in the cross-sectional analysis (Figure 4) and the observation of fairly similar progression in different individuals, apparently showing largely different onset ages (Figure 3).

A

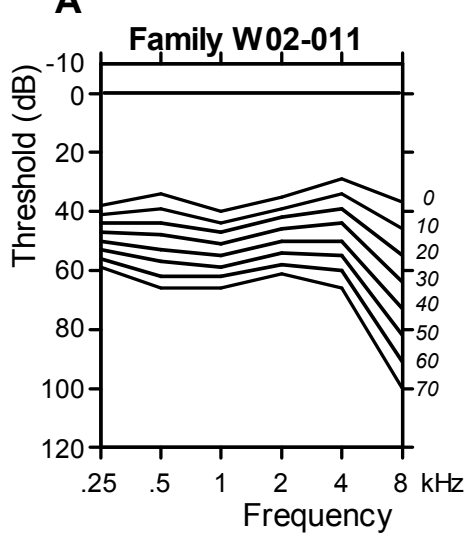

B

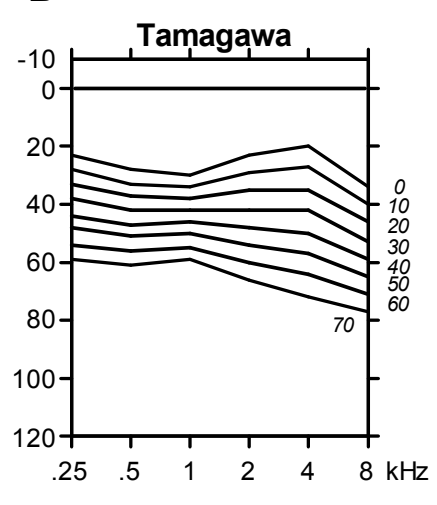

C

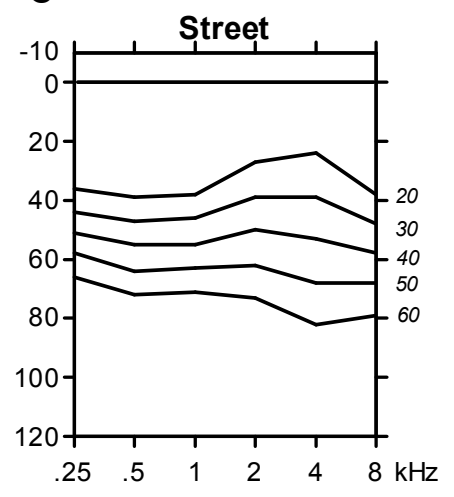

Figure 5. ARTA based on cross-sectional analyses of the present family (A) and derived from cross-sectional data reported for the Japanese and the American DFNA11 families (B and $\mathrm{C}$ ), respectively. Italics indicate age in decade steps. Thin lines in panels A and B indicate absence of threshold estimated age in panel C.

Figure 5 shows the ARTA derived from the cross-sectional analyses of the present family (Figure 5A), together with the ARTA based on the cross-sectional data of the Japanese family (Figure 5B) and the American family (Figure 5C). ${ }^{23,26}$ The results of attempted longitudinal analyses were fairly comparable to those of the cross-sectional analysis in these families, but the numbers of longitudinal data were limited. The ARTA of the present family (Figure 5A) show a fairly flat threshold curve at young ages and only modest progression, most clearly at the high frequencies. The ARTA of the Japanese family (Figure 5B) are quite similar to that of the Dutch family, with less progression in the high frequencies. The ARTA of the American family (Figure 5C) are also fairly similar, with slightly more progression. Speech recognition was remarkably well preserved in the present family, with a score of $50 \%$ correct still present at 80 years of age. From an onset age (i.e. $90 \%$ score) of 36 years onwards, the speech recognition score declined at a deterioration rate of $0.9 \%$ per year (Figure $6 \mathrm{~A}$ ). Figure $6 \mathrm{~B}$ shows the binaural mean speech recognition score as a function of binaural mean PTA1,2, kHz, with an onset level (i.e. $90 \%$ score) of $51 \mathrm{~dB}$ HL, decreasing by $0.8 \%$ per $\mathrm{dB}$. Speech recognition scores in the present family were comparable to those reported for the Japanese family; only the deterioration gradients were significantly different according to ANCOVA (Figure 6, Table 1). There were no reports on speech recognition in the other families. 

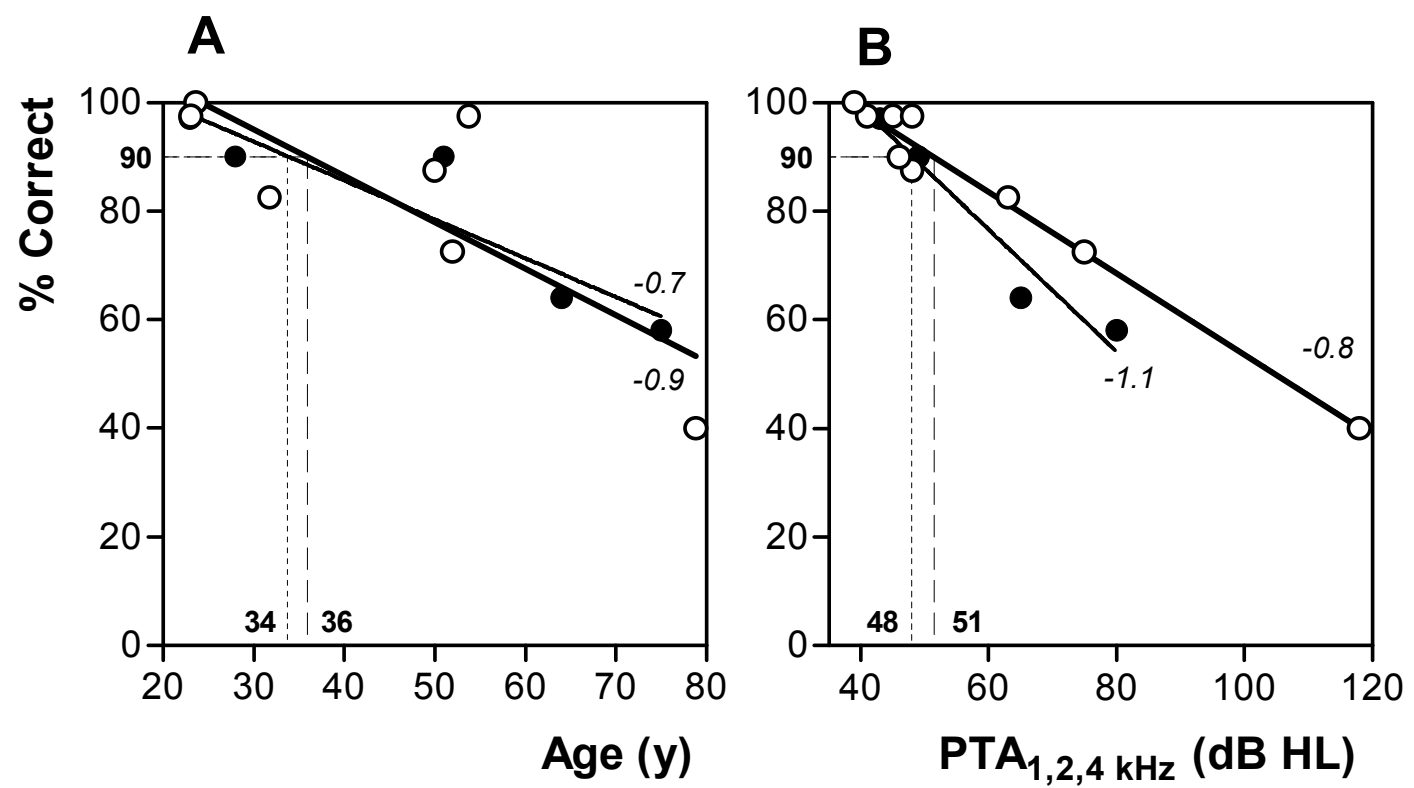

Figure 6. Cross-sectional binaural mean speech recognition scores (\% correct) of the Dutch and Japanese families at last visit against age (in years) (A) and binaural mean

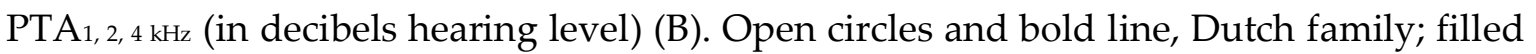
circles and thin line, Japanese family. The dashed (Dutch family) or dotted (Japanese family) lines with bold number indicate onset age (A) and onset level (B). Numbers near the regression line indicate deterioration rate $(\mathrm{A})$ and deterioration gradient $(\mathrm{B})$.

Table 1. Comparison of clinical and genetic data of all DFNA11 families

\begin{tabular}{|c|c|c|c|c|}
\hline & $\begin{array}{l}\text { Present family }{ }^{25} \\
\text { nr: W02-011 }\end{array}$ & Tamagawa et al. 23,24 & Bolz et al. ${ }^{27}$ & $\begin{array}{l}\text { Street et al. }{ }^{26} \\
\text { nr: HL2 }\end{array}$ \\
\hline Nucleotide change & Asn458Ile & Ala886_Lys888del & Arg853Cys & Gly722Arg \\
\hline Location & Motor domain & Coiled coil region & IQ5 domain & Motor domain \\
\hline Onset age (y) & $4-43$ & $12-16$ & Childhood & $20-30$ \\
\hline Audiogram & 1.f. $\rightarrow$ flat $\rightarrow$ downsloping & Flat $\rightarrow$ downsloping & Not available & 1.f. $\rightarrow$ flat $\rightarrow$ downsloping \\
\hline ATD/progression & $0.3-0.9 \mathrm{~dB}$ per $\mathrm{y}^{*}$ & mean: $0.56 \mathrm{~dB}$ per $\mathrm{y}^{*}$ & Slow & Present \\
\hline Vestibular symptoms & 4 of 6 patients & Absent & Not available & Absent \\
\hline Vestibular dysfunction & 4 of 6 patients & 3 of 5 patients & 1 of 5 patients & Not available \\
\hline Retinal dysfunction & Subclinical & Absent & Absent & Absent \\
\hline $\begin{array}{l}\text { Speech: } \\
\text { Age of onset (y) }\end{array}$ & 36 & 34 & & \\
\hline Deterioration rate $(\% / y)$ & 0.9 & 0.7 & & \\
\hline Level of onset (dB HL) & 51 & 48 & & \\
\hline $\begin{array}{l}\text { Deterioration gradient } \\
\text { (\% per } \mathrm{dB} \mathrm{HL})\end{array}$ & 0.8 & 1.0 & & \\
\hline
\end{tabular}

del, deletion; l.f., low frequency; $\mathrm{nr}$, number; $\mathrm{y}$, years; $\mathrm{dB}$, decibel; $\mathrm{HL}$, hearing level; ${ }^{*}$, derived from cross-sectional data. 


\section{Vestibular examination}

Vestibular examination was performed in six mutation carriers, aged 23 to 78 years. Two individuals had no vestibular symptoms and normal vestibular responses (III:5 and III:22). Vestibular symptoms, such as vertigo and unsteady walking, especially in the dark, were present in four individuals. Spontaneous nystagmus was not observed. The oldest person (II:1) showed vestibular areflexia. Remarkably, only two years previously she had had a vertigo attack for the first time. The youngest person (IV:4, proband) showed mild hyporeflexia of the velocity-step response, with borderline low caloric responses. Hyporeflexia of the rotatory responses with bilateral caloric weakness was found in the other two individuals, III:1 and III:3, of which the former had more severe and asymmetric vestibular dysfunction.

\section{Ocular examinations}

Ocular symptoms were not reported. Nobody complained about night blindness. The results of ocular function tests are presented in Table 2. Corrected visual acuity was subnormal in carrier II:1, caused by cataract. Intraocular pressures and keratometry readings were normal. Ophthalmoscopy showed little dots of pigmentation in the fundus periphery in one person (III:5), but no bone-spicule pigmentations were present. Signs of typical retinitis pigmentosa, such as arteriolar attenuation and optic disc pallor, were not found. Goldmann perimetry showed a generally decreased sensitivity in individual II:1 and an enlarged blind spot in three persons (III:3, III:5 and IV:4). Also, individual III:5 had a relative scotoma in the temporal visual fields of both eyes. A contraction of both nasal visual fields was seen in individual IV:4.

Table 2. Ocular examination of 5 mutation carriers of the present family

\begin{tabular}{|c|c|c|c|c|c|c|c|c|}
\hline $\begin{array}{l}\text { Mutation } \\
\text { Carrier; } \\
\text { (sex/age) }\end{array}$ & Eye & $\begin{array}{l}\text { Visual } \\
\text { Acuity }\end{array}$ & Fundus & Visual field & $\begin{array}{l}\text { EOG } \\
\text { Lp/Dt }\end{array}$ & Cone-ERG & Rod-ERG & Conclusion \\
\hline $\begin{array}{l}\text { II-1 } \\
\text { E, } 80 y\end{array}$ & $\begin{array}{l}\mathrm{R} \\
\mathrm{L}\end{array}$ & $\begin{array}{l}0.8 \\
0.6\end{array}$ & $\begin{array}{l}\text { Normal } \\
\text { Normal }\end{array}$ & $\begin{array}{l}\downarrow \text { Sensitivity } \\
\downarrow \text { Sensitivity }\end{array}$ & $\begin{array}{l}1.8 \\
1.6\end{array}$ & $\begin{array}{l}\text { Normal } \\
\text { Normal }\end{array}$ & $\begin{array}{l}\text { Normal } \\
\text { Normal }\end{array}$ & $\begin{array}{l}\text { Mild RPE involvement } \\
\text { Mild RPE involvement }\end{array}$ \\
\hline $\begin{array}{l}\text { III: } 1 \\
\text { M, } 54 \text { y }\end{array}$ & $\begin{array}{l}\mathrm{R} \\
\mathrm{L}\end{array}$ & $\begin{array}{l}0.8 \\
1.2\end{array}$ & $\begin{array}{l}\text { Normal } \\
\text { Normal }\end{array}$ & $\begin{array}{l}\text { Normal } \\
\text { Normal }\end{array}$ & $\begin{array}{l}2.1 \\
2.1\end{array}$ & $\begin{array}{l}\text { Normal } \\
\text { Subnormal }\end{array}$ & $\begin{array}{l}\text { Subnormal } \\
\text { Subnormal }\end{array}$ & $\begin{array}{l}\text { PR involvement } \\
\text { PR involvement }\end{array}$ \\
\hline $\begin{array}{l}\text { III:3 } \\
\text { M, } 53 \text { y }\end{array}$ & $\begin{array}{l}\mathrm{R} \\
\mathrm{L}\end{array}$ & $\begin{array}{l}1.0 \\
1.0\end{array}$ & $\begin{array}{l}\text { Normal } \\
\text { Normal }\end{array}$ & $\begin{array}{l}\uparrow \text { Blind spot } \\
\uparrow \text { Blind spot }\end{array}$ & $\begin{array}{l}1.9 \\
1.9\end{array}$ & $\begin{array}{l}\text { Normal } \\
\text { Normal }\end{array}$ & $\begin{array}{l}\text { Normal } \\
\text { Normal }\end{array}$ & $\begin{array}{l}\text { Normal } \\
\text { Normal }\end{array}$ \\
\hline $\begin{array}{l}\text { III:5 } \\
\text { M, } 51 \text { y }\end{array}$ & $\begin{array}{l}\mathrm{R} \\
\mathrm{L}\end{array}$ & $\begin{array}{l}1.0 \\
1.0\end{array}$ & $\begin{array}{l}\text { Pigment } \\
\text { Normal }\end{array}$ & $\begin{array}{l}\text { Temporal scotoma } \\
\text { Temporal scotoma }\end{array}$ & $\begin{array}{l}1.9 \\
2.2\end{array}$ & $\begin{array}{l}\text { Abnormal } \\
\text { Abnormal }\end{array}$ & $\begin{array}{l}\text { Abnormal } \\
\text { Abnormal }\end{array}$ & $\begin{array}{l}\text { PR involvement } \\
\text { PR involvement }\end{array}$ \\
\hline $\begin{array}{l}\text { IV:4 } \\
\text { F, } 24 y\end{array}$ & $\begin{array}{l}\mathrm{R} \\
\mathrm{L}\end{array}$ & $\begin{array}{l}1.0 \\
1.2\end{array}$ & $\begin{array}{l}\text { Normal } \\
\text { Normal }\end{array}$ & $\begin{array}{l}\uparrow \text { Blind spot } \\
\uparrow \text { Blind spot }\end{array}$ & $\begin{array}{l}2.7 \\
2.5\end{array}$ & $\begin{array}{l}\text { Normal } \\
\text { Normal }\end{array}$ & $\begin{array}{l}\text { Normal } \\
\text { Normal }\end{array}$ & $\begin{array}{l}\text { Normal } \\
\text { Normal }\end{array}$ \\
\hline
\end{tabular}


The EOG light peak/dark trough ratio was abnormal $(<1.9)$ in one person (II:1), corresponding to mild involvement of the retinal pigment epithelial cells. The ERG results were subnormal in two individuals (III:1 and III:5). This might suggest subclinical involvement of retinal photoreceptor cells in these two brothers, however, typical retinitis pigmentosa was not seen.

\section{Discussion}

The clinical features of a Dutch family with a MYO7A mutation causing DFNA11 are presented in this study. Hearing impairment was symmetric and progressed by 0.2 to $2.6 \mathrm{~dB}$ per year according to the longitudinal analyses. Cross-sectional analysis showed a more constant, milder progression over all frequencies of 0.3 to $0.9 \mathrm{~dB}$ per year. Subjective onsets varied between childhood and 43 years of age, with one exception of age 63 years. Variability in onset age and severity was seen across affected family members. Speech recognition scores were relatively good, with an onset age of 36 years and a deterioration rate of $0.9 \%$ per year. Recognition scores decreased by $0.8 \%$ per $\mathrm{dB}$ threshold increase, from a PTA $1,2,4$ kHz of $51 \mathrm{~dB}$ HL onward.

Comparison of the data of the present DFNA11 family with those reported for the other DFNA11 families showed pure-tone and speech audiometric data to be fairly similar. The range in onset ages was relatively wide compared with the other families (Table 1). Variability in onset age and severity across affected family members was observed in the present and the American family. ${ }^{25,26}$ As compared with USH1B and DFNB2, sensorineural hearing impairment was mild to moderate in all families, with minor progression. The audiometric configuration varies between fairly flat to gently downsloping, or more steeply downsloping at advanced ages in all three available families. In addition, some members of the present and of the American family (initially) showed lowfrequency characteristics. ${ }^{25,26}$ Four members of the present family reported having tinnitus. In the other families, only one Japanese mutation carrier had tinnitus. ${ }^{24}$ All three sets of ARTA were fairly similar, based on the comparison of crosssectional analyses. ${ }^{24,26}$ The American family seemed to have a slightly higher progression, which was most pronounced at the high frequencies. ${ }^{26}$

Vestibular dysfunction in the present family is not fully penetrant. It varied from normal function in two persons (aged 42 and 50 years) to complete vestibular areflexia in one person (aged 79 years). It seems likely that vestibular impairment is progressive, but the number of observations was too small to confirm this. In the other families, only one person had vestibular symptoms. ${ }^{27}$ Tamagawa et al. 
reported no vestibular symptoms. Nevertheless, three of five patients had spontaneous nystagmus and caloric hypofunction. ${ }^{23,24}$ In the German family, mild vestibular dysfunction was found in one person. ${ }^{27}$ Thus, in the present family, more pronounced vestibular failure has been recorded than in the other DFNA11 families.

Ocular symptoms, including night blindness, were not reported in the present family. Ophthalmoscopy showed little dots of pigmentation in the fundus periphery in one person. Goldmann perimetry was abnormal in four individuals. The EOG light peak/dark trough ratio was abnormal in one person and ERG values were subnormal in two individuals. These findings might suggest subclinical involvement of both retinal pigment epithelial cells and retinal photoreceptor cells. Similar findings were not reported in the other families. In the Japanese family, visual acuity tests, ophthalmoscopy, intraocular pressure measurements and slit-lamp examination were performed on five carriers and ERG on four affected persons. In the American family, fundus examination was performed on two mutation carriers and in the German family ERG, fundus examination and dark adaptation studies were performed on four affected persons. ${ }^{26,27}$ Because of the limited data, subclinical signs of retinal abnormalities that remained unnoticed in the other families cannot be excluded.

In the present family, a 1373 A-to-T transversion in exon 13 was found that leads to an Asn458Ile substitution in the switch II $\alpha$-helix of myosin VIIa. ${ }^{25} \mathrm{~A}$ molecular model of myosin VIIa, based on the structure of myosin II heavy chain from Dictyostelium discoideum, predicted the Asn458Ile substitution to be responsible for the hearing impairment. The differently shaped side chain of isoleucine at position 458 is presumed to cause important structural changes in the motor head domain. This is believed to disrupt ATP/ADP binding and/or impair the power stroke. ${ }^{25}$

The Japanese DFNA11 family $7,23,24,35$ carries an in-frame-9-bp deletion in exon 22 of MYO7A, leading to the loss of three amino acids (Ala886_Lys888del) in the coiledcoil domain of the protein. ${ }^{8}$ In the American family, a c. $2164 \mathrm{G}>\mathrm{C}$ mutation is present in exon 17, leading to a Gly722Arg nucleotide substitution in the motor domain of the protein. ${ }^{26}$ In the German family, a c. $2557 \mathrm{C}>\mathrm{T}$ mutation in exon 21 causes a substitution of cysteine for arginine at residue 853 in the fifth IQ motif. ${ }^{27}$ Although the four different mutations in MYO7A affect three different domains of the expressed protein, the clinical presentation of the families is fairly similar. This is difficult to explain with regard to what is known about the function of these protein domains. 
Up to now, a dominant negative effect was suggested through, for example, improper dimerization of the aberrant protein in the Japanese family ${ }^{23}$ and through correct dimerization that also impairs the function of the wild-type proteins. ${ }^{25-27}$ One might suggest that all DFNA11 mutations reduce the stability of the protein, as suggested by the results of Rhodes et al. for the headbanger mouse. However, the reduction in levels of myosin VIIa ${ }^{36}$ does not explain why carriers of truncating mutations that are causative for Usher syndrome Type 1B have no symptoms and signs of hearing impairment, ${ }^{37}$ unless dimerization with mutant myosin VIIa causes a dominant negative effect. This would then result in less than $50 \%$ of the normal protein level causing cochleovestibular and retinal dysfunction, whereas $50 \%$ of normal protein causes no dysfunction in carriers of Usher syndrome Type $1 \mathrm{~B}$.

It is interesting to compare the clinical presentations of DFNA11 and USH1B. Usher syndrome Type I is characterized by congenital profound hearing impairment, with sparing only of residual hearing at the lower frequencies, ${ }^{38}$ together with vestibular areflexia and prepubertal retinitis pigmentosa. ${ }^{39}$ DFNA11 patients have mild to moderate progressive hearing impairment and vestibular dysfunction associated with subclinical retinal abnormalities in the present family. Carriers of Usher Type 1B showed ophthalmologic abnormalities as well, but had normal hearing. ${ }^{37,40}$ However, the genetic diagnosis was based on gene linkage results, as mutation analysis on $M Y O 7 A$ was not performed by that time.

\section{Conclusion}

In summary, we found fairly similar cochleovestibular phenotype in all DFNA11 families with subclinical retinal abnormalities exclusively in the present family. Investigations with in vivo models will be needed to reveal the mechanisms involved in DFNA11 and myosin VIIa.

\section{References}

1. Van Camp G, Smith RJH. Hereditary Hearing Loss Homepage. URL: http://dnalabwww.uia.ac.be/dnalab/hhh/. Accessed June, 2005.

2. Strom T, Hortnagel K, Hofmann S, Gekeler F, Scharfe C, Rabl W, Gerbitz K, Meitinger T. Diabetes insipidus, diabetes mellitus, optic atrophy and deafness (DIDMOAD) caused by mutations in a novel gene (wolframin) coding for a predicted transmembrane protein. Hum Mol Genet 1998;7:2021-2028.

3. Bespalova I, Van Camp G, Bom SJH, Brown D, Cryns K, DeWan A, Erson A, Flothmann K, Kunst HPM, Kurnool P, Sivakumaran T, Cremers CWRJ, Leal S, Burmeister M, Lesperance M. Mutations in the Wolfram syndrome 1 gene (WFS1) are a common cause of low frequency sensorineural hearing loss. Hum Mol Genet 2001;10:2501-2508. 
4. Young T, Ives E, Lynch E, Person R, Snook S, MacLaren L, Cater T, Griffin A, Fernandez B, Lee $\mathrm{M}$, King M, Cator T. Non-syndromic progressive hearing loss DFNA38 is caused by heterozygous missense mutation in the Wolfram syndrome gene WFS1. Hum Mol Genet 2001;10:3111.

5. Verhoeven K, Van Laer L, Kirschhofer K, Legan PK, Hughes DC, Schatteman I. Mutations in the human alpha-tectorin gene cause autosomal dominant non-syndromic hearing impairment. Nat Genet 1999;21:449.

6. Mustapha M, Weil D, Chardenoux S, Elias S, El-Zir E, Beckmann JS. An alpha-tectorin gene defect causes a newly identified autosomal recessive form of sensorineural pre-lingual nonsyndromic deafness, DFNB21. Hum Mol Genet. 1999;8:409-412.

7. Tamagawa Y, Kitamura K, Ishida T, Ishikawa K, Tanaka H, Tsuji S, Nishizawa M. A gene for a dominant form of non-syndromic sensorineural deafness (DFNA11) maps within the region containing the DFNB2 recessive deafness gene. Hum Mol Genet 1996;5:849-852.

8. Liu XZ, Walsh J, Tamagawa Y, Kitamura K, Nishizawa M, Steel KP, Brown SD. Autosomal dominant non-syndromic deafness caused by a mutation in myosin VIIA gene. Nat Genet 1997;17:268-269.

9. Liu XZ, Walsh J, Mburu P, Kendrick-Jones J, Cope MJ, Steel KP, Brown SD. Mutations in the myosin VIIA gene cause non-syndromic recessive deafness. Nat Genet 1997;16:188-190.

10. Liu XZ. The clinical presentation of DFNB2. In: Cremers CWRJ, Smith RJH (eds). Genetic Hearing Impairment. Its clinical presentations. Advances in Oto-Rhino-Laryngology, vol. 61. Basel: Karger, 2002;61:120-123.

11. Weil D, Blanchard S, Kaplan J, Guilford P, Gibson F, Walsh J, Mburu P, Varela A, Levilliers J, Weston MD, Kelley PM, Kimberling WJ, Wagenaar M, Levi-Acobas F, Larget-Piet D, Munnich A, Steel KP, Brown SDM, Petit C. Defective myosin VIIA gene responsible for Usher syndrome type 1B. Nature 1995;374:60-61.

12. Liu XZ, Hope C, Walsh J, Newton V, Ke XM, Liang CY, Xu LR, Zhou JM, Trump D, Steel KP, Bundey S, Brown SD. Mutations in the myosin VIIA gene cause a wide phenotypic spectrum, including atypical Usher syndrome. Am J Hum Genet 1998;63:909-912.

13. Ben Zina Z, Masmoudi S, Ayadi H, Chaker F, Ghorbel AM, Drira M, Petit C. From DFNB2 to Usher syndrome: variable expressivity of the same disease. Am J Med Genet 2001;101:181-183.

14. Hasson T, Heintzelman MB, Santos-Sacchie J, Corey DP, Mooseker MS. Expression in cochlea and retina of myosin VIIa, the gene product defective in Usher syndrome type $1 \mathrm{~B}$. Proc Natl Acad Sci USA 1995;92:9815-9819.

15. Hasson T, Gillespie PG, Garcia JA, MacDonald RB, Zhao Y, Yee AG, Mooseker MS, Corey DP. Unconvential myosins in inner-ear sensory epithelia. J Cell Biol 1997;137:1287-1307.

16. Wolfrum U, Liu X, Schmitt A, Udovichenko IP, Williams DS. Myosin VIIa as a common component of cilia and microvilli. Cell Motil Cytoskel 1998;40:261-271.

17. El-Amraoui A, Sahly I, Picaud S, Sahel J, Abitbol M, Petit C. Human Usher 1B/mouse shaker-1: the retinal phenotype discrepancy explained by the presence/absence of myosin VIIA in the photoreceptor cells. Hum Mol Genet 1996;5:1171-1178.

18. Liu X, Vansant G, Udovichenko IP, Wolfrum U, Williams DS. Myosin VIIa, the product of the Usher $1 \mathrm{~B}$ syndrome gene, is concentrated in the connecting cilia of photoreceptor cells. Cell Motil Cytoskel 1997;37:240-252.

19. Gibbs D, Azarian SM, Lillo C, Kitamoto J, Klomp AE, Steel KP, Libby RT, Williams DS. Role of myosin VIIa and Rab27a in the motility and localization of RPE melanosomes. J Cell Sci 2004;117:6473-6483.

20. Liu X, Udovichenko IP, Brown SDM, Steel KP, Williams DS. Myosin VIIa participates in opsin transport through the photoreceptor cilium. J Neurosci 1999;19:6267-6274.

21. Boëda B, El-Amraoui A, Bahloul A, Goodyear R, Daviet L, Blanchard S, Perfettini I, Fath KR, Shorte S, Reiners J, Houdusse A, Legrain P, Wolfrum U, Richardson G, Petit C. Myosin VIIa, 
harmonin and cadherin 23 , three Usher I gene products that cooperate to shape the sensory hair cell bundle. EMBO J 2002;21:6689-6699.

22. Adato A, Michel V, Kikkawa Y, Reiners J, Alagramam KN, Weil D, Yonekawa H, Wolfrum $\mathrm{U}$, El-Amraoui A, Petit C. Interactions in the network of Usher syndrome type 1 proteins. Hum Mol Genet 2005;14:347-356.

23. Tamagawa Y, Ishikawa K, Ishida T, Kitamura K, Makino S, Tsuru T, Ichimura K. Phenotype of DFNA11: a nonsyndromic hearing loss caused by a myosin VIIA mutation. Laryngoscope 2002;112:292-297.

24. Tamagawa $Y$, Ishikawa K, Ishikawa K, Ishida T, Kitamura K, Makino S, Tsuru T, Ichimura K. Clinical presentation of DFNA11 (MYO7A). In: Cremers CWRJ, Smith RJH (eds). Genetic Hearing Impairment. Its clinical presentations. Advances in Oto-Rhino-Laryngology, vol. 61. Basel: Karger, 2002;61:79-84.

25. Luijendijk MWJ, van Wijk E, Bischoff AMLC, Krieger E, Huygen PLM, Pennings RJE, Brunner HG, Cremers CWRJ, Cremers FPM, Kremer H. Identification and molecular modeling of a mutation in the motor head domain of myosin VIIA in a family with autosomal dominant hearing impairment (DFNA11). Hum Genet 2004;115:149-156.

26. Street VA, Kallman JC, Kiemele KL. J Med Genet 2004; Modifier controls severity of a novel dominant low-frequency MyosinVIIA (MYO7A) auditory mutation. J Med Genet 2004; 41: e62.

27. Bolz H, Bolz SS, Schade G, Kothe C, Mohrmann G, Hess M, Gal A. Impaired calmodulin binding of myosin-7A causes autosomal dominant hearing loss (DFNA11). Hum Mutat 2004;24:274-275.

28. Auletta G, Ciccodicola A, Cubellis MV, D'Adamo AP, D'Eustacchio A, Di Leva F, Donaudy F, Errichiello M, Saulino C, Franze A, Gasparini P, Marciano F. A novel mutation in the Myosin VIIA motor domain in a family with autosomal dominant hearing loss (DFNA11). Presented at the meeting "Genes, Hearing and Deafness. From Molecular Biology to Clinical Practice". Caserta, Italy, March 17-19, 2005.

29. Marres HAM, van Ewijk M, Huygen PLM, Kunst HPM, Van Camp G, Coucke P, Cremers CWRJ. Inherited nonsyndromic hearing loss. An audiovestibular study in a large family with autosomal dominant progressive hearing loss related to DFNA2. Arch Otolaryngol Head Neck Surg 1997;123:573-577.

30. Huygen PLM, Pennings RJE, Cremers CWRJ. Characterizing and distinguishing progressive phenotypes in nonsyndromic autosomal dominant hearing impairment. Audiol Med 2003; 1:37-46.

31. Bom SJH, De Leenheer EMR, Lemaire FX, , Kemperman MH, Verhagen WIM, Marres HAM, Kunst HPM, Ensink RJH, Bosman AJ, Van Camp G, Cremers CWRJ, Huygen PLM. Speech recognition scores related to age and degree of hearing impairment in DFNA2/KCNQ4 and DFNA9/COCH. Arch Otolaryngol Head Neck Surg 2001;127:1045-1048.

32. Marmor MF, Zrenner E. Standard for clinical electro-oculography. International Society for Clinical Electrophysiology of Vision. Doc Ophthalmol 1993;85:115-124.

33. Marmor MF, Zrenner E. Standard for clinical electroretinography (1999 update). International Society for Clinical Electrophysiology of Vision. Doc Ophthalmol 1998-99;97: 143-156.

34. ISO 7029. Acoustics. Threshold as hearing by air conduction as a function of age and sex for otologically normal persons. Geneva: International Organisation for Standardization, 1984.

35. Tamagawa Y, Kitamura K, Ishida T, Nishizawa M, Liu X-Z, Walsh J, Steel KP, Brown SDM. Sensorineural hearing impairment, non-syndromic, dominant DFNA11. In: Kitamura K, Steel KP (eds). Genetics in Otorhinolaryngology. Advances in Oto-Rhino-Laryngology, vol. 56. Basel: Karger 2000;56:103-106. 
36. Rhodes CR, Hertzano R, Fuchs H, Bell RE, de Angelis MH, Steel KP, Avraham KB. A Myo7a mutation cosegregates with stereocilia defects and low-frequency hearing impairment. Mamm Genome 2004;15:686-697.

37. Wagenaar M, Snik AF, Kimberling WJ, Cremers CWRJ. Carriers of the Usher syndrome type IB: is audiometric identification possible? Am J Otol 1996;17:853-858.

38. Wagenaar M, van Aarem A, Huygen PLM, Pieke-Dahl S, Kimberling WJ, Cremers CWRJ. Hearing impairment related to age in Usher syndrome types 1B and 2A. Arch Otolaryngol Head Neck Surg 1999;125:441-445.

39. Kimberling WJ, Möller C. Clinical and molecular genetics of Usher syndrome. J Am Acad Audiol 1995;6:63-72.

40. Wagenaar M, ter Rahe B, van Aarem A, Huygen PLM, Admiraal RJC, Bleeker-Wagemakers E, Pinckers A, Kimberling WJ, Cremers CWRJ. Clinical findings in obligate carriers of type I Usher syndrome. Am J Med Genet 1995;59:375-379. 



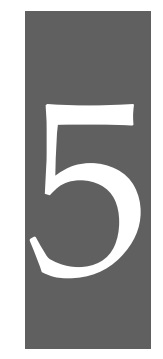

Discussion and Summary 



\section{Discussion}

The principal aim of this thesis was to contribute to our knowledge on phenotypegenotype correlation in autosomal dominant types of non-syndromic hearing impairment: DFNA5, DFNA9 and DFNA11. In the chapters of this thesis the clinical features of the investigated families have been described in relation to the genotypes. Other families, in which genetic analysis has not been successful yet, are not included in this thesis. Two new mutations have been identified in the DFNA5 and the DFNA11 families, of which the latter has been reported by Luijendijk et al. ${ }^{1}$ Detailed audiometric analyses of the DFNA5, DFNA9 and DFNA11 families have been made and they have been compared with other families described in the literature, where possible. It is very important to evaluate if the phenotypes are (dis)similar in families with different mutations in the same gene for a more specific diagnosis and better counselling.

In chapter 2, a DFNA5 family is described and the phenotype has been compared to that of the original family. ${ }^{2}$ The phenotype of a third DFNA5 family was not available during the preparation of this article for a distinct comparison with the other families. ${ }^{3}$ All three known families showed a similar phenotype with progressive sensorineural hearing impairment starting in the high frequencies, with better speech discrimination in the family described in the present article. Although the mutations are different in all families, they have the same effect on the mRNA, namely skipping of exon 8 . This suggests that these DFNA5 mutations are "gain of function" mutations that lead to a truncated protein with a deleterious novel function. Studies by Van Laer et al. $^{4}$ supported this by demonstrating significantly increased necrotic cell death in mammalian HEK293T cells transfected with mutant DFNA5 compared to cells transfected with wild-type DFNA5. Although DFNA5 expression is present at low levels in multiple tissues, only progressive hearing loss occurs in mutation carriers. This can be explained by a larger susceptibility of cochlear cells to the damaging actions of mutant DFNA5, because DFNA5 might interact with cellular components that are only expressed in cochlear cells. ${ }^{4}$

The cochleovestibular phenotype of DFNA9 patients with the Pro51Ser mutation in the $\mathrm{COCH}$ gene is comparable. Hearing impairment develops from the fourth to fifth decade, similar to the phenotype of the following mutations in the $\mathrm{COCH}$ gene: Gly87Trp, Gly88Glu, Val104del, Ile109Asn, Ile109Thr, Ala119Thr, and Cys542Phe. ${ }^{5-13}$ In contrast, it starts in the second to third decades in the American families with the Val66Gly and Trp117Arg mutations.,14 Different from other 
COCH mutations, ${ }^{11,12}$ the vestibular dysfunction starts almost a decade before the hearing impairment begins in patients with the Pro51Ser mutation.

In chapter 4, a DFNA11 family has been described and the phenotype has been compared to that of three other DFNA11 families. ${ }^{15-18}$ A fifth family was not described at the time. ${ }^{19}$ Although the mutations in myosin VIIa were different in all families, the inner ear phenotype was similar. Patients suffered from sensorineural hearing impairment with steady progression at all frequencies and vestibular dysfunction. However, the vestibular function was intact in the family described by Street et al. ${ }^{18}$

In DFNA9 and DFNA11 two ophthalmologic features are described that have not been observed before. The subtle subclinical retinal abnormalities that are shown in the investigated DFNA11 family might be faint reflections of retinitis pigmentosa, which is seen in Usher syndrome Type IB. Mutations in the same gene also cause DFNB2, in which no retinal abnormalities are present. It is still unclear how deficiency of functional myosin VIIa leads to retinal dysfunction in Usher Type 1B and the present DFNA11 family and why there are no retinal features in DFNB2 and in the other three known DFNA11 families described in the literature. Retinal degeneration has not been diagnosed in the shaker-1 mouse model and the Usher Type 1B rat model (Myo7a $\left.\mathrm{a}^{\text {tnd-1Hubr }}\right)^{20,21}$ However, some retinal abnormalities have been found, such as a decreased amplitude in electroretinography of shaker-1 mice, ${ }^{22}$ abnormal opsin accumulation in the connecting cilium and reduction of photoreceptive disk membrane renewal rate in shaker-1 mice ${ }^{23}$ and absence of melanosomes in the apical processes of the retinal pigment epithelium in both animal models. ${ }^{21,24}$ Further investigations will be necessary to clarify this subject, especially in the headbanger mice with an autosomal dominant mutation Myo7a were no attention was paid to possible retinal features. ${ }^{25}$

The characteristic vertical corneal striae found in three DFNA9 families with the Pro51Ser and the Gly88Glu mutation in the $\mathrm{COCH}$ gene cannot easily be explained. We propose that this corneal feature and the cochleovestibular dysfunction are caused by these two mutations in the $\mathrm{COCH}$ gene. This is supported by the detection of co-deposits of cochlin and acidophilic mucopolysaccharide in the trabecular meshwork of patients with primary open angle glaucoma and in glaucomatous DBA/2J mice with progressive hearing loss. ${ }^{26,27}$ Other evidence to support the causative relationship is the identification of COCH transcripts in the human cornea using RT-PCR (Chapter 3.2) and in a cDNA library constructed using RNA from the anterior segment of the zebrafish 
eye. ${ }^{28}$ Additional investigations of the other DFNA9 families with different mutations will be required to strengthen this theory.

Hereditary non-syndromic hearing impairment is quite heterogeneous. New types of hereditary non-syndromic hearing impairment have been reported almost every month. About 125 non-syndromic autosomal dominant and autosomal recessive types have been identified in approximately 15 years and by now, 39 causative genes are known. The increasing understanding of the genes involved will provide more and more insight into the molecular mechanisms that underly the processes of normal hearing and hearing impairment. The genes known today encode a variety of proteins, including extracellular matrix proteins, motor molecules, gap junction proteins, transcription factors and ion channels. The function of several proteins known to be involved in sensorineural hearing impairment is still unknown, such as DFNA5 (in DFNA5), wolframin (in DFNA6/14/38 and Wolfram syndrome) and TMC1 (in DFNA36).

With the completion of the Human Genome Project in 2003, the field of genetic medicine is experiencing an essential transformation. The identification of our genes and the proteins they encode and the knowledge of the pathologic mechanisms of the mutations will contribute tremendously, the developments in diagnosis, treatment and prevention of hereditary diseases. Such knowledge will make gene therapy possible for hearing impaired patients in the future, but for now, we only have hearing aids and cochlear implants to offer. Gene therapy comprises the introduction of normal genes into human somatic or stem cells to treat a specific disorder. Because the cochlea is a very small organ, which is difficult to reach, the development of a successful gene delivery method is challenging. There are non-viral vectors, such as liposomes, and viral vectors, for instance adenovirus, adeno-associated virus, herpes simplex virus and lentivirus vectors. ${ }^{29}$ Viral vectors use their infectivity to launch and express the exogenous genes that have been inserted in the viral genome. Adenovirus vectors are being used most widely at this moment. It is effective at many sites of the cochlea. An unfortunate side effect is a strong immune response, which can cause a toxic effect on the recipient cell. This can, however, be treated by immunosuppression. ${ }^{29} \mathrm{An}$ additional problem is the limited duration of expression, because the adenovirus does not integrate in the human genome. ${ }^{29}$ It is important to develop a method that delivers vectors into the cochlea without damaging the cochlear architecture, causing hearing impairment. Several methods have been examined, including osmotic minipump infusion or microinjection into the scala tympani via the round window $w^{30-32}$ or through a cochleostomy in the basal turn of the cochlea ${ }^{33,34}$ and 
injection into the endolymphatic sac and even into the posterior semicircular canal. ${ }^{34,35}$ The least invasive technique has been application of gelfoam soaked with therapeutic agent to the round window membrane. ${ }^{36}$

Another difficulty for gene therapy is synthesis and accumulation of aberrant proteins, which causes irreversible damage. This occurs in particular in autosomal dominant types of hearing impairment. To prevent this, gene therapy at an early stage would be necessary. Additionally, if there is a dominant negative mechanism, the expression of the mutant protein has to be stopped, to make gene therapy successful. Maeda et al. have succeeded to silence the expression of the mutated GJB2 gene in vivo using short interfering RNA, which protects normal hearing. ${ }^{37}$

Gene therapy is more easily achieved with autosomal recessive diseases, where the insertion of a normal gene will replace the absent product. It has been effectively applied to the mouse model of DFNB3, the shaker-2 mouse model. Insertion of a bacterial artificial chromosome transgene containing the Myo15a gene into homozygous shaker-2 mice maintains normal cochlear structure and function and excess of Myo15a does not induce a toxic effect. ${ }^{38}$

Stem cell therapy is another challenge. Mammals cannot regenerate auditory hair cells as lower vertebrates can. Genomic tools are being used with the aim to decipher the molecular basis of this regenerative capability. For hearing restoration it is needed to not only produce functional hair cells, but also to integrate them into appropriate neural pathways. Another challenge is to structure the cell growth and prevent uncontrolled production of cells leading to tumour growth. It is clear that it will take many years before gene therapy on the inner ear will be applied in a clinical setting.

\section{Summary}

Chapter 1 begins with a general introduction of hereditary hearing impairment.

In table 1, the phenotype and genotype of all known DFNA-types are described, together with all identified mutations. Finally, an up to date review is given on DFNA5, DFNA9 and DFNA11.

Chapter 2 describes the clinical and genetic evaluation of a Dutch DFNA5 family with non-syndromic sensorineural hearing impairment and compares the clinical data with the first Dutch DFNA5 family. ${ }^{2}$ Because our article was published almost simultaneously with that of the Chinese DFNA5 family, ${ }^{3}$ we could not compare these two families. Sensorineural hearing impairment was non- 
syndromic and symmetric with predominance in the high frequencies. Subjective hearing impairment arose from 0 to 40 years. Audiometric analyses showed a congential hearing loss of $30 \mathrm{~dB}$ in the high frequencies. The hearing loss was most progressive at 0.5 and $1 \mathrm{kHz}$. Pure-tone and speech audiometric data were compared to the data of the first DFNA5 family. ${ }^{2}$ The present DFNA5 family has similar hearing impairment as the original family in terms of pure-tone thresholds, but with more favourable speech. Due to resemblance in audiometric data with the first DFNA5 family, linkage analysis was found with markers flanking the DFNA5 gene. Mutation analysis revealed the presence of a DFNA5 mutation causing a C-to-G nucleotide transversion in the splice acceptor site of intron 7. This leads to skipping of exon 8 in part of the transcripts.

The relatively low amount of the aberrant gene transcript in the present family suggests that a dominant negative effect of the mutant protein causes the hearing loss. Because the abnormal splicing product has been found in higher levels in the first family and is also present at very low levels in controls, there might be a critical threshold for the aberrant protein above which it becomes pathogenic. Whether the differences in speech recognition between the two families are due to the different ratios of normal versus aberrant protein remains to be clarified.

Chapter 3 presents cochleovestibular and ocular features of DFNA9. In Chapter 3.1 a clinical report on cochleovestibular impairment of a family with the Pro51Ser mutation in the $\mathrm{COCH}$ gene has been described, after which a detailed analysis has been made of 74 mutation carriers with the same gene defect. The vestibuloocular reflex, plotted against age, was compared with the pure tone average at 1,2 and $4 \mathrm{kHz}$, plotted against the same parameter. Vestibular dysfunction appeared to have a $100 \%$ penetrance. We concluded that vestibular impairment starts about nine years earlier, progresses more rapidly and eventually, is more complete than hearing impairment in Pro51Ser mutation carriers. At the time that the hearing impairment had just started, which is at the age of 43 years, the vestibular dysfunction was already almost complete.

In Chapter 3.2 a significant association of DFNA9 and peculiar vertical corneal striae is described in three DFNA9 families with two different mutations in the $\mathrm{COCH}$ gene. The examinations were performed on a total of 98 family members with 61 mutation carriers of four families. Families 1 (Chapter 3.1) and 2 harbour the Pro51Ser mutation and families 3 and 4 harbour the Gly88Glu and the Gly87Trp mutation, respectively. The vertical corneal striae caused minor problems, as dry eye symptoms, and were not present in the general Dutch 
ophthalmologic population. The striae were present from an age of 47 years in 32 individuals, of whom 27 individuals had a $\mathrm{COCH}$ mutation. Statistical analysis on the striae and the $\mathrm{COCH}$ mutations showed a significant association in families 1 , 2 and 3, but not in family 4 . Although five individuals without the mutation have the vertical cornea striae and there is no association in family 4 , a sequence variant in a neighbouring gene causing cosegregation of the two features is unlikely. Because the striae are present in three DFNA9 families with two different $\mathrm{COCH}$ mutations and the vertical corneal striae are not present in the ophthalmologic population, we propose that the Pro51Ser and the Gly88Glu mutations in the $\mathrm{COCH}$ gene cause both the vertical corneal striae and the cochleovestibular dysfunction. This is supported by the detection of transcripts of the $\mathrm{COCH}$ gene in the human cornea and in a cDNA library constructed from RNA of the anterior segment of the zebrafish eye. ${ }^{28}$ In addition, cochlin has been detected in the trabecular meshwork of patients with primary open angle glaucoma. Accumulation of this protein with eosinophilic glycosaminoglycans has been found in both the cochlea and the anterior part of the eye. ${ }^{26,39}$

A Dutch DFNA11 family is presented in Chapter 4. Mutation analysis revealed a c.1373A $>$ T nucleotide substitution in exon 13, that is predicted to cause an Asn458Ile amino acid substitution in the motor domain of myosin VIIa. ${ }^{1}$ Subjective hearing loss was initiated between the ages of four and 43 years. The hearing impairment pattern was flat or lightly downsloping. Subclinical abnormalities were found in Goldmann perimetry, electro-oculography and electroretinography. This points to subtle dysfunctions of the photoreceptor cells and retinal pigment epithelial cells in the retina. Cochleovestibular and ocular features were compared to data on three other DFNA11 families. ${ }^{15-18}$ A fifth family was not described at the time. ${ }^{19}$ Although the four known DFNA11 mutations in the MYO7A gene affect three different domains of the protein, the inner ear phenotype was found to be fairly similar in all DFNA11 families. Nevertheless, no retinal abnormalities were found in the other DFNA11 families. Mutations in MYO7A can cause Usher syndrome type IB, DFNB2 and DFNA11. In Usher syndrome type IB visual impairment due to retinitis pigmentosa is a main feature, together with congenital profound hearing loss. Retinal dysfunction has not been reported in DFNB2. Remarkably, delicate subclinical retinal involvement of both photoreceptor cells and retinal pigment epithelial cells was detected in the present DFNA11 family, while this was not found in the other DFNA11 families. 


\section{References}

1. Luijendijk MWJ, Van Wijk E, Bischoff AMLC, Krieger E, Huygen PLM, Pennings RJE, Brunner HG, Cremers CWRJ, Cremers FPM, Kremer H. Identification and molecular modeling of a mutation in the motor head domain of myosin VIIA in a family with autosomal dominant hearing impairment (DFNA11). Hum Genet 2004;115:149-156.

2. De Leenheer EMR, van Zuijlen DA, Van Laer L, Van Camp G, Huygen PLM, Huizing EH, Cremers CWRJ. Clinical features of DFNA5. ). In: Cremers CWRJ, Smith RJH (eds). Genetic Hearing Impairment. Its clinical presentations. Advances in Oto-Rhino-Laryngology, vol. 61. Basel: Karger, 2002;61:53-59.

3. Yu C, Meng X, Zhang S, Zhao G, Hu L, Kong X. A 3-nucleotide deletion in the polypyrimidine tract of the intron 7 of the DFNA5 gene causes nonsyndromic hearing impairment in a Chinese family. Genomics 2003;82: 575-579.

4. Van Laer L, Vrijens K, Thys S, Van Tendeloo VF, Smith RJH, Van Bockstaele DR, Timmermans JP, Van Camp G. DFNA5: hearing impairment exon instead of hearing impairment gene? J Med Genet 2004;41:401-406.

5. Robertson NG, Lu L, Heller S, Eavey RD, McKenna M, Nadol JB Jr, Miyamoto RT, Linthicum FH Jr, Lubianca Neto JF, Hudspeth AJ, Seidman CE, Morton CC, Seidman JG. Mutations in a novel cochlear gene cause DFNA9, a human nonsyndromic deafness with vestibular dysfunction. Nat Genet 1998;20:299-303.

6. De Kok YJM, Bom SJH, Brunt TM, Kemperman MH, van Beusekom E, van der Velde-Visser SD, Robertson NG, Morton CC, Huygen PLM, Verhagen WIM, Brunner HG, Cremers CWRJ, Cremers FPM. A Pro51Ser mutation in the $\mathrm{COCH}$ gene is associated with late onset autosomal dominant progressive sensorineural hearing loss with vestibular defects. Hum Mol Genet 1999;8:361-366.

7. Fransen E, Verstreken M, Verhagen WIM, Wuyts FL, Huygen PLM, D'Haese P, Robertson NG, Morton CC, McGuirt WT, Smith RJH, Declau F, Van de Heyning PH, Van Camp G. High prevalence of symptoms of Menière's disease in three families with a mutation in the COCH gene. Hum Mol Genet 1999;8:1425-1429.

8. Kamarinos M, McGill J, Lynch M, Dahl H. Identification of a novel COCH mutation, I109N, highlights the similar clinical features observed in DFNA9 families. Hum Mutat 2001;17:351. Erratum in: Hum Mutat 2001;18:547-548.

9. Usami S, Takahashi K, Yuge I, Ohtsuka A, Namba A, Abe S, Fransen E, Patthy L, Otting G, Van Camp G. Mutations in the $\mathrm{COCH}$ gene are a frequent cause of autosomal dominant progressive cochleo-vestibular dysfunction, but not of Meniere's disease. Eur J Hum Genet 2003;11:744-748.

10. Nagy I, Horvath M, Trexler M, Répássy G, Patthy L. A novel COCH mutation, V104del, impairs folding of the LCCL domain of cochlin and causes progressive hearing loss. J Med Genet 2004;41:e9

11. Kemperman MH, De Leenheer EMR, Huygen PLM, van Duijnhoven G, Morton CC, Robertson NG, Cremers FPM, Kremer H, Cremers CWRJ. Audiometric, vestibular and genetic aspects of a DFNA9 family with a G88E COCH mutation. Otol Neurotol 2005;26:926933.

12. Pauw RJ, Collin RWJ, Huygen PLM, Hoefsloot LH, Kremer H, Cremers CWRJ. Clinical characteristics of a Dutch DFNA9 family with a novel $\mathrm{COCH}$ mutation, G87W. Audiol Neurootol 2007;12:77-84.

13. Pauw RJ, Huygen PLM, Collin RWJ, Cruysberg JRM, Hoefsloot LHS, Kremer H, Cremers CWRJ. Phenotype description of a novel DFNA9/COCH mutation, I109T. Annals of Otology, Rhinology and Laryngology. In press.

14. Khetarpal U, Schuknecht HF, Gacek RR, Holmes LB. Autosomal dominant sensorineural hearing loss. Pedigrees, audiologic findings, and temporal bone findings in two kindreds. Arch Otolaryngol Head Neck Surg 1991;117:1032-1042. 
15. Tamagawa Y, Ishikawa K, Ishida T, Kitamura K, Makino S, Tsuru T, Ichimura K. Phenotype of DFNA11: a nonsyndromic hearing loss caused by a myosin VIIA mutation. Laryngoscope 2002;112:292-297.

16. Tamagawa $Y$, Ishikawa K, Ishikawa K, Ishida T, Kitamura K, Makino S, Tsuru T, Ichimura K. Clinical presentation of DFNA11 (MYO7A). ). In: Cremers CWRJ, Smith RJH (eds). Genetic Hearing Impairment. Its clinical presentations. Advances in Oto-Rhino-Laryngology, vol. 61. Basel: Karger, 2002;61:79-84.

17. Street VA, Kallman JC, Kiemele KL. J Med Genet 2004; Modifier controls severity of a novel dominant low-frequency MyosinVIIA (MYO7A) auditory mutation. J Med Genet 2004; 41: e62.

18. Bolz H, Bolz SS, Schade G, Kothe C, Mohrmann G, Hess M, Gal A. Impaired calmodulin binding of myosin-7A causes autosomal dominant hearing loss (DFNA11). Hum Mutat 2004;24:274-275.

19. Di Leva F, D'Adamo P, Cubellis MV, D'Eustacchio A, Errichiello M, Saulino C, Auletta G, Giannini P, Donaudy F, Ciccodicola A, Gasparini P, Franze A, Marciano E. Identification of a Novel Mutation in the Myosin VIIA Motor Domain in a Family with Autosomal Dominant Hearing Loss (DFNA11). Audiol Neurootol 2006;11:157-164.

20. Hasson T, Walsh J, Cable J, Mooseker MS, Brown SD, Steel KP. Effects of shaker-1 mutations on myosin-VIIa protein and mRNA expression.Cell Motil Cytoskeleton 1997;37:127-138.

21. Smits BM, Peters TA, Mul JD, Croes HJ, Fransen JA, Beynon AJ, Guryev V, Plasterk RH, Cuppen E. Identification of a rat model for usher syndrome type $1 \mathrm{~B}$ by N-ethyl-Nnitrosourea mutagenesis-driven forward genetics. Genetics 2005;170:1887-1896.

22. Libby RT, Steel KP. Electroretinographic anomalies in mice with mutations in Myo7a, the gene involved in human Usher syndrome type 1B. Invest Ophthalmol Vis Sci 2001;42:770778.

23. Liu X, Udovichenko IP, Brown SDM, Steel KP, Williams DS. Myosin VIIa participates in opsin transport through the photoreceptor cilium. J Neurosci 1999;19:6267-6274.

24. Liu X, Ondek B, Williams DS. Mutant myosin VIIa causes defective melanosome distribution in the RPE of shaker-1 mice. Nat Genet 1998;19:117-118.

25. Rhodes CR, Hertzano R, Fuchs H, Bell RE, de Angelis MH, Steel KP, Avraham KB. A Myo7a mutation cosegregates with stereocilia defects and low-frequency hearing impairment. Mamm Genome 2004;15:686-697.

26. Bhattacharya SK, Rockwood EJ, Smith SD, Bonilha VL, Crabb JS, Kuchtey RW, Robertson NG, Peachey NS, Morton CC, Crabb JW. Proteomics reveal cochlin deposits associated with glaucomatous trabecular meshwork. J Biol Chem 2005;280:6080-6084.

27. Bhattacharya SK, Annangudi SP, Salomon RG, Kuchtey RW, Peachey NS, Crabb JW. Cochlin deposits in the trabecular meshwork of the glaucomatous DBA/2J mouse. Exp Eye Res 2005;80:741-744.

28. Vihtelic TS, Fadool JM, Gao J, Thornton KA, Hyde DR, Wistow G. Expressed sequence tag analysis of zebrafish eye tissues for NEIBank. Mol Vis 2005;11:1083-1100.

29. Lalwani AK, Jero J, Mhatre AN. Developments in cochlear gene therapy. ). In: Cremers CWRJ, Smith RJH (eds). Genetic Hearing Impairment. Its clinical presentations. Advances in Oto-Rhino-Laryngology, vol. 61. Basel: Karger, 2002;61:28-33.

30. Raphael Y, Frisancho JC, Roessler BJ. Adenoviral-mediated gene transfer into guinea pig cochlear cells in vivo. Neurosci Lett 1996;207:137-141.

31. Komeda M, Roessler BJ, Raphael Y. The influence of interleukin-1 receptor antagonist transgene on spiral ganglion neurons. Hear Res 1999;131:1-10.

32. Kho ST, Pettis RM, Mhatre AN, Lalwani AK. Cochlear microinjection and its effects upon auditory function in the guinea pig. Eur Arch Otorhinolaryngol 2000;257:469-472.

33. Carvalho GJ, Lalwani AK. The effect of cochleostomy and intracochlear infusion on auditory brain stem response threshold in the guinea pig. Am J Otol 1999;20:87-90. 
34. Kawamoto K, Oh SH, Kanzaki S, Brown N, Raphael Y. The functional and structural outcome of inner ear gene transfer via the vestibular and cochlear fluids in mice. Mol Ther 2001;4:575-585.

35. Yamasoba T, Yagi M, Roessler BJ, Miller JM, Raphael Y. Inner ear transgene expression after adenoviral vector inoculation in the endolymphatic sac. Hum Gene Ther 1999;10:769-774.

36. Jero J, Mhatre AN, Tseng CJ, Stern RE, Coling DE, Goldstein JA, Hong K, Zheng WW, Hoque AT, Lalwani AK. Cochlear gene delivery through an intact round window membrane in mouse. Hum Gene Ther 2001;12:539-548.

37. Maeda Y, Fukushima K, Nishizaki K, Smith RJH. In vitro and in vivo suppression of GJB2 expression by RNA interference. Hum Mol Genet 2005;14:1641-1650.

38. Kanzaki S, Beyer L, Karolyi IJ, Dolan DF, Fang Q, Probst FJ, Camper SA, Raphael Y. Transgene correction maintains normal cochlear structure and function in 6-month-old Myo15a mutant mice. Hear Res 2006;214:37-44.

39. Robertson NG, Cremers CWRJ, Huygen PLM, Ikezono T, Krastins B, Kremer H, Kuo SF, Liberman MC, Merchant SN, Miller CE, Nadol JB Jr, Sarracino DA, Verhagen WI, Morton CC. Cochlin immunostaining of inner ear pathologic deposits and proteomic analysis in DFNA9 deafness and vestibular dysfunction. Hum Mol Genet 2006;15:1071-1085. 



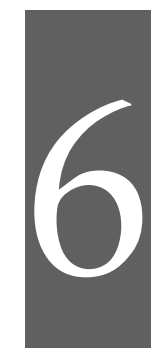

Samenvatting 



\section{Samenvatting}

Het hoofddoel van dit proefschrift is het ontwikkelen van kennis over het klinisch beeld bij families met een bepaald type niet-syndromale autosomaal dominant overervende slechthorendheid, bekend als "DFNA": DFNA5, DFNA9 en DFNA11. Audiometrische en vestibulaire data werden verzameld en geanalyseerd om zo specifiek mogelijk het fenotype te karakteriseren. Bloedmonsters werden genomen voor genkoppeling-onderzoek en mutatieanalyse om het genotype te identificeren. Tevens werden andere klinische kenmerken zoals spraakherkenning onderzocht. Bij DFNA9 en DFNA11 werd ook oogheelkundig onderzoek verricht om mogelijk aanwezige subklinische afwijkingen te kunnen opsporen. Vervolgens werden de klinische data vergeleken met de data van andere families met hetzelfde DFNA-type. De zorgvuldige beschrijving van het fenotype in vergelijking met het genotype is van belang voor een goede classificatie en klinische diagnostiek van erfelijke slechthorendheid. Het bespaart tijd en daarmee kosten om voor een bekend locus genkoppeling en/of mutatie-analyse te kunnen toepassen op basis van de klinische diagnostiek, zodat een volledige diagnostische screening van het genoom niet nodig is. Er is een enorme toename ontstaan in kennis over de verschillende genen die betrokken zijn bij slechthorendheid en de heterogeniteit van erfelijk gehoorverlies is aanzienlijk. Het bestuderen van de klinische kenmerken is daarmee van betekenis, om op basis van het fenotype het vermoedelijke genotype te leren kennen. In de laatste 15 jaar zijn er maar liefst 54 loci en 21 genen geïdentificeerd voor nietsyndromale, autosomaal dominant overervende slechthorendheid. Een verdere uitbreiding van het aantal DFNA loci tot wel 100 is in de nabije toekomst te verwachten. Een ander klinisch belang van deze studies is om patiënten die aan een erfelijke aandoening lijden en hun familieleden nauwkeurig en uitgebreid te kunnen informeren. Al deze kennis over de betrokken genen, de functie van het eiwit en het pathologische mechanisme veroorzaakt door de mutaties, zal een buitengewone bijdrage leveren aan behandeling en preventie van erfelijke aandoeningen in de toekomst.

Hoofdstuk 1 is een algemene inleiding over erfelijke niet-syndromale slechthorendheid. In tabel 1 wordt het fenotype en genotype van alle autosomaal dominante vormen van slechthorendheid vermeld. Dit is de meest "actuele" tabel waarin ook alle mutaties staan vermeld. Vervolgens wordt een overzicht gegeven over DFNA5, DFNA9 en DFNA11. 
Hoofdstuk 2 beschrijft de klinische en genetische evaluatie van een DFNA5 familie met niet-syndromale sensorineurale slechthorendheid en vergelijkt de klinische data met de originele DFNA5 familie. ${ }^{1}$ Tijdens het schrijven van het artikel was de derde DFNA5 familie uit China nog niet gepubliceerd, waardoor de data van deze familie niet geanalyseerd konden worden. ${ }^{2}$ Het fenotype is een progressieve en symmetrische slechthorendheid van aanvankelijk alleen de hoge tonen. Het subjectieve gehoorverlies ontstaat tussen de leeftijd van nul tot 40 jaar. Een statistische analyse van audiometrische gegevens maakt een congenitaal gehoorverlies in de hoge tonen aannemelijk, van zo'n $30 \mathrm{~dB}$. Op $0.5 \mathrm{kHz}$ en $1 \mathrm{kHz}$ is het gehoorverlies het meest progressief. Analyse van toon- en spraakaudiometrische data van de twee DFNA5 families toont een zelfde fenotype wat betreft de toonaudiometrie, met beter behoud van de spraakdiscriminatie in de huidige familie.

Genkoppeling-onderzoek van deze tweede DFNA5 familie werd direct opgestart, omdat de audiometrische data vergelijkbaar waren met de originele DFNA5 familie. Dit bevestigt opnieuw het belang van fenotype-genotype vergelijking. Een volledige genoomscan was onnodig, waardoor tijd en kosten werden bespaard. De mutatie bevat een nucleotide verandering in de splice-acceptor site van intron 7, dat afwezigheid van exon 8 in een deel van de transcripten veroorzaakt. Aangezien er maar een kleine hoeveelheid van het afwijkend gentranscript wordt aangetroffen in de huidige familie, lijkt de aanwezigheid van een dominant negatief effect waarschijnlijk. Dit houdt een type mutatie in waarin het afwijkende eiwit een complex vormt met het normale eiwit, zodat het niet meer functioneert. In de originele familie wordt een hoger percentage abnormaal genprodukt gevonden en ook in de controles is een, zei het zeer kleine, hoeveelheid aberrant eiwit aanwezig. Het lijkt derhalve alsof er een kritieke drempel bestaat, waarboven dit afwijkend proteïne schadelijk wordt. De vraag is nog onbeantwoord of de verschillen in spraakdiscriminatie tussen de twee DFNA5 families worden veroorzaakt door de verschillende verhoudingen tussen normaal en afwijkend eiwit.

In Hoofdstuk 3 wordt het cochleovestibulaire en oogheelkundige fenotype van DFNA9 beschreven. In Hoofdstuk 3.1 wordt aandacht besteed aan de cochleovestibulaire disfunctie in een familie met de bekende Nederlands-Vlaamse founder mutatie in het $\mathrm{COCH}$ gen (Pro51Ser). Vervolgens wordt een gedetailleerde analyse gemaakt van 74 mutatiedragers van de Pro51Ser mutatie, inclusief de huidige familie. De vestibulo-oculaire reflex, uitgezet tegen de leeftijd, wordt vergeleken met de gemiddelde toondrempel genomen van 1, 2 en $4 \mathrm{kHz}$, 
uitgezet tegen dezelfde parameter. Uit deze gegevens kan geconcludeerd worden dat de vestibulaire disfunctie $100 \%$ penetrant is en dat deze ongeveer negen jaar vooruitloopt op het gehoorverlies. De afname van de evenwichtsfunctie is tevens progressiever en uiteindelijk vollediger dan de slechthorendheid in patiënten met de Pro51Ser mutatie. Op 43 jaar is het gehoorverlies net ingetreden terwijl de vestibulaire disfunctie al bijna compleet is.

Hoofdstuk 3.2 spitst zich toe op de vondst van typische verticale striae in het anterieure segment van de cornea, in dezelfde familie als wordt beschreven in hoofdstuk 3.1. Vervolgens zijn nog drie DFNA9 families oogheelkundig onderzocht om te achterhalen of er een mogelijk oorzakelijk verband bestaat tussen verticale cornea striae en verschillende mutaties in het $\mathrm{COCH}$ gen. Families 1 (Hoofdstuk 3.1) en 2 bezitten de Pro51Ser mutatie, en families 3 en 4 respectievelijk de Gly88Glu en de Gly87Trp mutatie. In totaal zijn 98 familieleden onderzocht, waarvan 61 mutatiedragers zijn. De verticale cornea striae zijn enkel zichtbaar na aanbrengen van fluoresceïne. Ze veroorzaken slechts klachten als droge en branderige ogen en fluctuerende visus, waardoor het aanpassen van een bril of lenzen zeer moeizaam is. De striae zijn een niet eerder gekend klinisch verschijnsel in Nederland. De striae zijn aanwezig in 32 familieleden, waarvan 27 mutatiedragers, vanaf een leeftijd van 47 jaar. Statistische analyse vond een significante associatie tussen verticale cornea striae en de $\mathrm{COCH}$ mutaties in de Pro51Ser mutatie in families 1 en 2 en de Gly88Glu mutatie in familie 3. In familie 4 is geen associatie aanwezig. Hoewel vijf personen zonder een mutatie de striae ook vertonen en er geen associatie wordt gevonden in familie 4, is het naar onze mening niet waarschijnlijk dat een sequentievariant in een aangrenzend gen de co-segregatie veroorzaakt. Aangezien de striae aanwezig zijn in drie DFNA9 families met twee verschillende mutaties in het $\mathrm{COCH}$ gen en de striae niet zichtbaar zijn in de oogheelkundige populatie, veronderstellen we dat de verticale cornea striae en de cochleovestibulaire disfunctie veroorzaakt worden door de Pro51Ser en de Gly88Glu mutaties in het $\mathrm{COCH}$ gen. Dit wordt ondersteund door de detectie van $\mathrm{COCH}$ gen transcripten in de humane cornea en in een cDNA bibliotheek geproduceerd van RNA van het anterieure oogsegment in zebravissen. ${ }^{3}$ Cochline, het genprodukt van $\mathrm{COCH}$, is bovendien gevonden in het trabekelsysteem in patiënten met primair open hoek glaucoom en in DBA/2J muizen met glaucoom en progressief gehoorverlies., ${ }^{4,5}$ Agglomeraten van dit eiwit met eosinofiele glycosaminoglycanen zijn aangetroffen in de cochlea alsook in het anterieure gedeelte van het oog., 
In Hoofdstuk 4 wordt tenslotte een DFNA11 familie beschreven. Het genotype bestaat uit een c.1373A>T nucleotide verandering in exon 13 dat een Asn458Ile substitutie veroorzaakt in het motor domein van myosine VIIa. ${ }^{7}$ Het gehoorverlies start tussen de leeftijd van vier tot 43 jaar en het audiogram heeft een vlakke of licht afdalende curve. De familie is tevens oogheelkundig onderzocht, aangezien mutaties in het myosine VIIa gen ook Usher syndroom type 1 veroorzaken, wat gekenmerkt wordt door vroegkinderlijke doofheid in combinatie met gezichtsverlies door progressieve retina dystrofie (retinitis pigmentosa). Subklinische afwijkingen zijn gevonden in deze DFNA11 familie bij gezichtsveldonderzoek, electroretinografie en electro-oculografie. Dit duidt op delicate afwijkingen ter hoogte van de fotoreceptor cellen en de pigment epitheel cellen van de retina. De cochleovestibulaire en oogheelkundige fenotypes zijn vergeleken met de data van drie andere DFNA11 families. ${ }^{8-11}$ Een vijfde DFNA11 familie is pas later gepubliceerd. ${ }^{12}$ Het cochleaire fenotype is in alle families praktisch hetzelfde, hoewel in elke familie een verschillende mutatie werd beschreven in wel drie verschillende domeinen van het myosine VIIa gen. Bij de andere in de literatuur gepubliceerde DFNA11 families werden verrassend genoeg geen afwijkingen van de retina gevonden. ${ }^{8-12}$ Mutaties in het myosine VIIa gen kunnen DFNA11, Usher syndroom type 1 en DFNB2 veroorzaken. In DFNB2 werden ook geen aanwijzingen gevonden voor retinitis pigmentosa. Het is onduidelijk waarom een afwijkend myosine VIIa eiwit leidt tot disfunctie in de retina in Usher syndroom type 1 en de beschreven DFNA11 familie, terwijl er geen afwijkingen zijn gevonden in de andere DFNA11 families of in DFNB2. In het shaker-1 muis model en het Usher type IB rat model (Myo7atnd-1Hubr) zijn ook geen ernstige aberraties in de retina gevonden. ${ }^{13,14}$ Vervolgonderzoeken zijn nodig om meer duidelijkheid te verschaffen over het pathofysiologisch mechanisme, zoals analyse van het oogheelkundig fenotype in de "headbanger" muizen. Deze knaagdieren bevatten een autosomaal dominante overervende mutatie in het Myo7a gen en zijn nog niet zorgvuldig oogheelkundig onderzocht. ${ }^{15}$ Mogelijk dat hernieuwd oogheelkundig onderzoek bij de eerder gepubliceerde DFNA11 families alsnog de door ons beschreven verfijnde oogheelkundige verschijnselen kan en zal aantonen.

\section{Referenties}

1. De Leenheer EMR, van Zuijlen DA, Van Laer L, Van Camp G, Huygen PLM, Huizing EH, Cremers CWRJ. Clinical features of DFNA5. ). In: Cremers CWRJ, Smith RJH (eds). Genetic Hearing Impairment. Its clinical presentations. Advances in Oto-Rhino-Laryngology, vol. 61. Basel: Karger, 2002;61:53-59. 
2. Yu C, Meng X, Zhang S, Zhao G, Hu L, Kong X. A 3-nucleotide deletion in the polypyrimidine tract of the intron 7 of the DFNA5 gene causes nonsyndromic hearing impairment in a Chinese family. Genomics 2003;82: 575-579.

3. Vihtelic TS, Fadool JM, Gao J, Thornton KA, Hyde DR, Wistow G. Expressed sequence tag analysis of zebrafish eye tissues for NEIBank. Mol Vis 2005;11:1083-1100.

4. Bhattacharya SK, Rockwood EJ, Smith SD, Bonilha VL, Crabb JS, Kuchtey RW, Robertson NG, Peachey NS, Morton CC, Crabb JW. Proteomics reveal cochlin deposits associated with glaucomatous trabecular meshwork. J Biol Chem 2005;280:6080-6084.

5. Bhattacharya SK, Annangudi SP, Salomon RG, Kuchtey RW, Peachey NS, Crabb JW. Cochlin deposits in the trabecular meshwork of the glaucomatous DBA/2J mouse. Exp Eye Res 2005;80:741-744.

6. Robertson NG, Cremers CWRJ, Huygen PLM, Ikezono T, Krastins B, Kremer H, Kuo SF, Liberman MC, Merchant SN, Miller CE, Nadol JB Jr, Sarracino DA, Verhagen WI, Morton CC. Cochlin immunostaining of inner ear pathologic deposits and proteomic analysis in DFNA9 deafness and vestibular dysfunction. Hum Mol Genet 2006;15:1071-1085.

7. Luijendijk MWJ, Van Wijk E, Bischoff AMLC, Krieger E, Huygen PLM, Pennings RJE, Brunner HG, Cremers CWRJ, Cremers FPM, Kremer H. Identification and molecular modeling of a mutation in the motor head domain of myosin VIIA in a family with autosomal dominant hearing impairment (DFNA11). Hum Genet 2004;115:149-156.

8. Tamagawa Y, Ishikawa K, Ishida T, Kitamura K, Makino S, Tsuru T, Ichimura K. Phenotype of DFNA11: a nonsyndromic hearing loss caused by a myosin VIIA mutation. Laryngoscope 2002;112:292-297.

9. Tamagawa $Y$, Ishikawa K, Ishikawa K, Ishida T, Kitamura K, Makino S, Tsuru T, Ichimura K. Clinical presentation of DFNA11 (MYO7A). ). In: Cremers CWRJ, Smith RJH (eds). Genetic Hearing Impairment. Its clinical presentations. Advances in Oto-Rhino-Laryngology, vol. 61. Basel: Karger, 2002;61:79-84.

10. Street VA, Kallman JC, Kiemele KL. J Med Genet 2004; Modifier controls severity of a novel dominant low-frequency MyosinVIIA (MYO7A) auditory mutation. J Med Genet 2004; 41: e62.

11. Bolz H, Bolz SS, Schade G, Kothe C, Mohrmann G, Hess M, Gal A. Impaired calmodulin binding of myosin-7A causes autosomal dominant hearing loss (DFNA11). Hum Mutat 2004;24:274-275.

12. Di Leva F, D'Adamo P, Cubellis MV, D'Eustacchio A, Errichiello M, Saulino C, Auletta G, Giannini P, Donaudy F, Ciccodicola A, Gasparini P, Franze A, Marciano E. Identification of a Novel Mutation in the Myosin VIIA Motor Domain in a Family with Autosomal Dominant Hearing Loss (DFNA11). Audiol Neurootol. 2006;11:157-164.

13. Hasson T, Walsh J, Cable J, Mooseker MS, Brown SD, Steel KP. Effects of shaker-1 mutations on myosin-VIIa protein and mRNA expression.Cell Motil Cytoskeleton 1997;37:127-138.

14. Smits BM, Peters TA, Mul JD, Croes HJ, Fransen JA, Beynon AJ, Guryev V, Plasterk RH, Cuppen E. Identification of a rat model for usher syndrome type $1 \mathrm{~B}$ by N-ethyl-N-nitrosourea mutagenesis-driven forward genetics. Genetics 2005;170:1887-1896.

15. Rhodes CR, Hertzano R, Fuchs H, Bell RE, de Angelis MH, Steel KP, Avraham KB. A Myo7a mutation cosegregates with stereocilia defects and low-frequency hearing impairment. Mamm Genome 2004;15:686-697. 



\section{Dankwoord}

Eindelijk is dit proefschrift klaar. Onderzoek doe je niet alleen en zonder de inzet van heel veel mensen zou dit proefschrift nooit tot stand zijn gekomen.

Als eerste wil ik professor Cremers bedanken. $U$ bent de drijvende kracht van dit proefschrift geweest en uw niet te stuiten enthousiasme is aanstekelijk. U gaf mij de mogelijkheid om een wetenschappelijke stage over erfelijke slechthorendheid te volbrengen en dankzij $\mathrm{u}$ heb ik het onderzoek kunnen voortzetten als promotie onderzoek. U heeft altijd tijd voor overleg en discussie. De zondag ochtenden op de koffie zijn memorabel.

Professor Cruysberg wil ik bedanken voor het verrichten van het oogheelkundig onderzoek, veelal in de weekenden. Ik ben $\mathrm{u}$ zeer dankbaar dat $\mathrm{u}$ mij wat inzicht heeft gegeven in de oogheelkunde en voor de tijd die $u$ voor mij hebt vrijgemaakt voor overleg.

Vervolgens een dankwoord voor mijn beide co-promotoren Hannie Kremer en Patrick Huygen. Beste Hannie, jij bent een grote steun voor mij geweest. Aan je onderbouwende kritieken heb ik zeer veel gehad. Dank voor al je hulp, zeker als de genetica me boven de pet ging. Beste Patrick, je kennis op het gebied van wiskunde, fysica en het binnenoor zijn van grote waarde voor mij geweest. Ik vond het heel bijzonder om met jou te kunnen brainstormen achter de computer. Vandaag is het precies 35 jaar geleden dat jij promoveerde!

Graag bedank ik ook de staf van de keel-, neus-, en oorheelkunde van het UMC St Radboud voor de kans die ze me hebben gegeven om te promoveren naast de opleiding tot keel-, neus-, en oorarts.

Ik ben de families die belangeloos aan dit onderzoek hebben meegewerkt zeer erkentelijk. Mijn dank gaat ook uit naar de medewerkers van het audiologisch centrum Nijmegen en Martien Nicolasen, voor de hulp bij het klinisch gedeelte van het onderzoek. Alle medewerkers van de afdeling Antropogenetica wil ik bedanken voor alle analyses. Mirjam, dank voor je belangrijke rol in hoofdstuk 2 . Diny Helsper-Peters, super dat je zo snel en zorgvuldig de lay-out hebt verzorgd!

Alle arts-assistenten KNO: Dirk, Capi, Steven, Bas, Karien, Martijn, Savitri, Erik, Niels, Brechtje, Ronald, Myrthe en Liselotte (Charlie's Angels), Stijn, Rutger, Bart, Godelieve, Olivier, Sylvia, Robert Jan, Jan-Willem, Ferdinand, Ilse, Anne-Martine 
en Veronique ook heel erg bedankt voor jullie steun en gezelligheid. Menige artsassistenten groep is toch wel een tikkeltje jaloers op de manier hoe wij met elkaar omgaan en de lol die we hebben op en buiten de werkvloer. Ik heb me het laatste jaar een beetje een afhaker gevoeld bij veel gebeurtenissen, maar dit zal ik dubbel en dwars goedmaken!

Lieve Liesbeth en Esmeralda, ik ben trots dat jullie mij als paranimfen willen bijstaan. Jullie hebben mij gesteund als ik me minder "okay" voelde en met jullie is het altijd lachen. Ik ben blij met jullie.

Al mijn vrienden wil ik bedanken voor alles wat ze voor me zijn. Ook al zie ik sommigen tijden niet (Ik kom nu zeker naar Ankara/Istanbul en Londen!), jullie betekenen zeer veel voor me!

Lieve oma, jij bent mijn "super granny"! Ik weet niet hoeveel kaarsjes je al voor me op hebt gestoken. Ik vind het geweldig dat ik je trouwfoto mocht gebruiken voor de voorkant.

Lieve papa en mama, bedankt voor jullie onvoorwaardelijke liefde, steun en vertrouwen. Jullie hebben Rose en mij altijd de ruimte gegeven om ons te ontplooien tot de mensen die we nu zijn. Dankjewel voor alles. Lieve Rose, fijn dat je er altijd voor me bent. Ik ben trots dat jij in maart de eindstreep hebt gehaald en wens je veel succes in toekomstige carrière. Verlies echter niet uit het oog dat er nog zoveel meer is dan werk.

Lieve Sander, C'EST FINI!!! Dank je wel voor je geduld en begrip. Fijn dat je er altijd voor me bent. Ik houd van je. Nu op naar een volgende fase................... 


\section{Curriculum Vitae}

Anne Bischoff werd op 21 januari 1977 geboren te Rotterdam. In 1995 behaalde zij het VWO eindexamen diploma aan het Drachtster Lyceum te Drachten. Na uitloting in Nederland startte zij in datzelfde jaar met de studie geneeskunde in Antwerpen, België. In juli 1998 behaalde zij het diploma kandidaat-arts met onderscheiding aan het Rijks Universitair Centrum Antwerpen (RUCA), waarna aan de doctoraalstudie werd begonnen. In december 2001 werd gestart met een wetenschappelijke stage over erfelijke slechthorendheid bij de afdeling keel-, neus- en oorheelkunde van het UMC St Radboud te Nijmegen. In juli 2002 werd het artsexamen behaald aan de Universitaire Instelling Antwerpen (UIA). In augustus 2002 werd het onderzoek naar erfelijke slechthorendheid voortgezet, wat uiteindelijk heeft geleid tot het tot stand komen van dit proefschrift. In augustus 2003 is zij begonnen met de opleiding keel-, neus- en oorheelkunde van het UMC St Radboud te Nijmegen. Het perifere deel van haar opleiding in het Rijnstate Ziekenhuis te Arnhem en het Canisius Wilhelmina Ziekenhuis te Nijmegen heeft ze onlangs afgerond. 

List of abbreviations

ADP adenosine diphosphate

ANCOVA analysis of covariance

ARTA age-related typical audiograms

ATD annual threshold deterioration

ATP adenosine triphosphate

BAEP brainstem auditory evoked potentials

Bp base pairs

dB decibels

cDNA complementary deoxyribonucleic acid

DNA deoxyribonucleic acid

DFN X-linked non-syndromic hearing loss

DFNA autosomal dominant inherited sensorineural hearing loss

DFNB autosomal recessive inherited sensorineural hearing loss

DFNY Y-linked inherited sensorineural hearing loss

DFNM modifier gene locus for hearing impairment

EOG electro-oculography

ERG electroretinography

HL hearing level

$\mathrm{Hz} \quad$ hertz

IOP intraocular pressure

IVS intervening sequence

$\mathrm{kHz} \quad$ kilo hertz

mRNA messenger ribonucleic acid

MMLV moloney Murine Leukemia Virus

NMD nonsense mediated decay

PTA pure tone average

RNA ribonucleic acid

RT-PCR reverse transcriptase polymerase chain reaction

$\mathrm{T} \quad$ time constant

USH Usher syndrome

VOR vestibulo-ocular reflex 
Finis coronat opus.

(De voltooiing kroont het werk) 